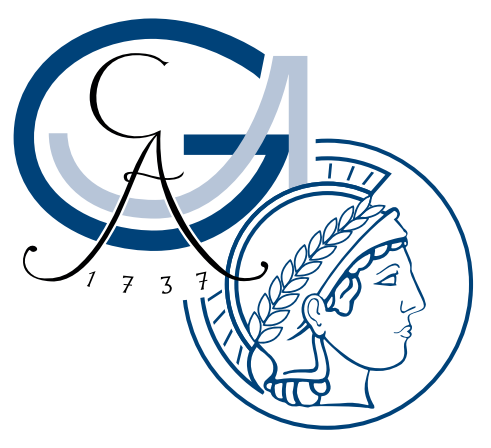

\title{
Functional analyses of RNA helicases in human ribosome biogenesis
}

\author{
Dissertation \\ for the award of the degree \\ "Doctor rerum naturalium" (Dr. rer. nat.) \\ of the Georg-August-Universität Göttingen \\ within the doctoral program Molecular Biology \\ of the Georg-August University School of Science (GAUSS) \\ submitted by
}

Priyanka Choudhury

from Kolkata, India

Göttingen 2019 


\section{Members of the Thesis Committee}

Prof. Dr. Markus Bohnsack ( $1^{\text {st }}$ reviewer)

Department of Molecular Biology, University Medical Center Göttingen

Prof. Dr. Marina Rodnina ( ${ }^{\text {nd }}$ reviewer)

Department of Physical Biochemistry, Max Planck Institute for Biophysical Chemistry, Göttingen

Prof. Dr. Jörg Stülke

Institute for Microbiology and Genetics, Georg-August-University Göttingen

Further members of the examination board

Prof. Dr. Ralf Ficner

Department of Molecular Structural Biology; Georg-August-University Göttingen

Prof. Dr. Michael Meinecke

Department of Cellular Biochemistry, University Medical Center Göttingen

Dr. Alexander Stein

Department of Membrane Protein Biochemistry, Max Planck Institute for Biophysical Chemistry, Göttingen

Date of oral examination: $12^{\text {th }}$ July 2019 


\section{Affidavit}

Herewith I declare that I prepared the PhD dissertation 'Functional analyses of RNA helicases in human ribosome biogenesis' on my own and with no other sources and aids than quoted.

Göttingen, May 2019

Priyanka Choudhury 


\section{Publications associated with the dissertation}

Priyanka Choudhury, Philipp Hackert, Indira Memet, Katherine E. Sloan \& Markus T. Bohnsack (2019). The human RNA helicase DHX37 is required for release of the U3 snoRNP from pre-ribosomal particles, RNA Biology, 16, 54-68. 


\section{Table of Contents}

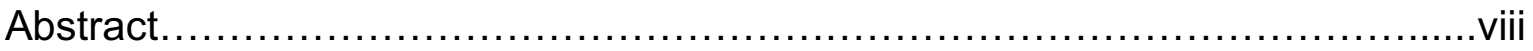

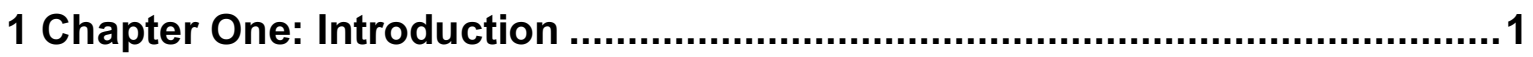

1.1 Overview of ribosome assembly pathway ..............................................1

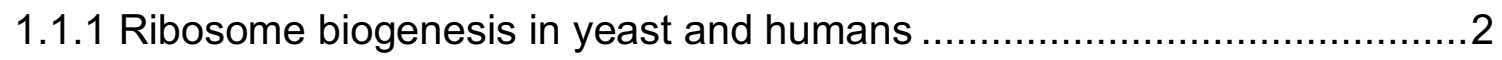

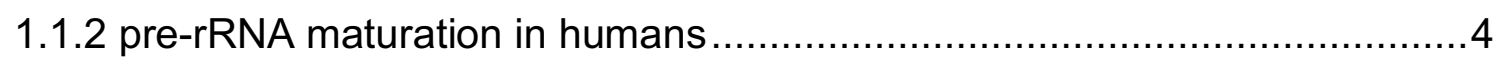

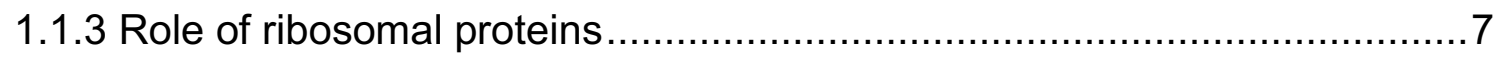

1.1.4 Structural transitions during subunit assembly in humans ...................... 8

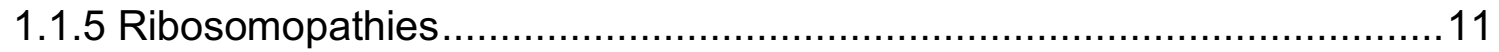

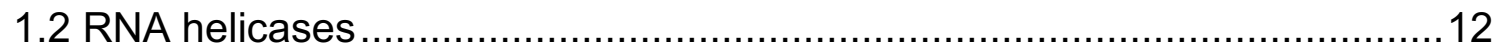

1.2.1 RNA helicase superfamilies and conserved sequence motifs ................12

1.2.2 Mechanism of action of RNA helicases ..............................................14

1.2.3 Cellular pathways involving RNA helicases ........................................16

1.2.3.1 Role of RNA helicases in pre-mRNA splicing .............................17

1.2.3.2 Role of RNA helicases in translation ........................................... 17

1.2.3.3 Role of RNA helicases in other cellular pathways ........................18

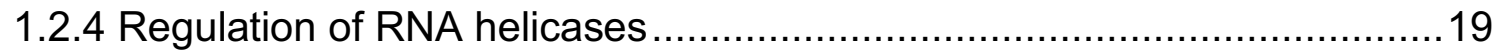

1.2.4.1 Cofactor-dependent regulation of RNA helicases......................... 19

1.2.4.2 Cofactor-independent regulation of RNA helicases.......................20

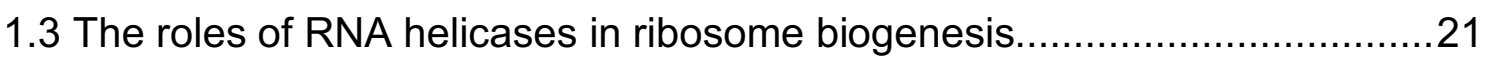

1.3.1 RNA helicases regulate snoRNA association with pre-ribosomal particles

1.3.2 RNA helicases mediate structural remodelling of pre-ribosomes .............23

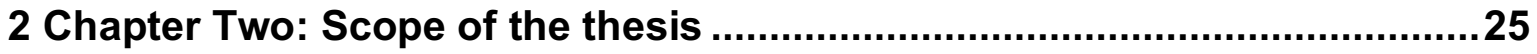

3 Chapter Three: Manuscript I .....................................................................26

4 Chapter Four: The role of DDX55 in human ribosome biogenesis ..............77

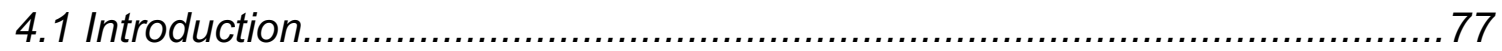

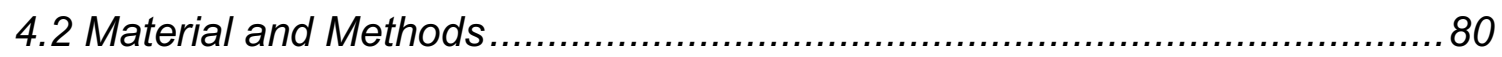

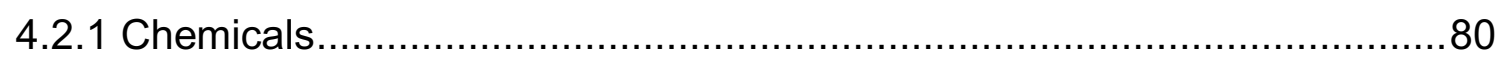

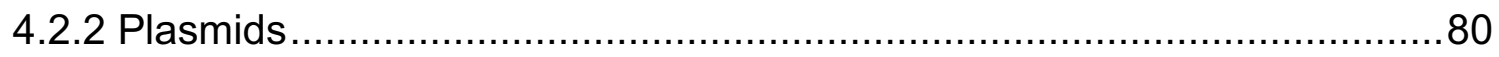




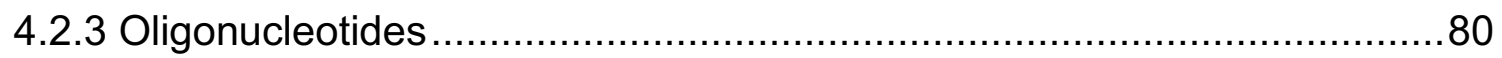

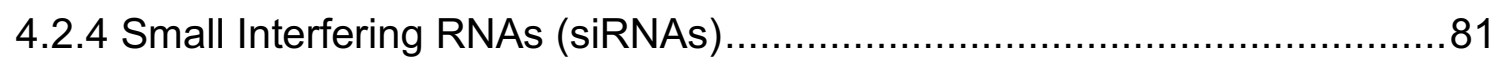

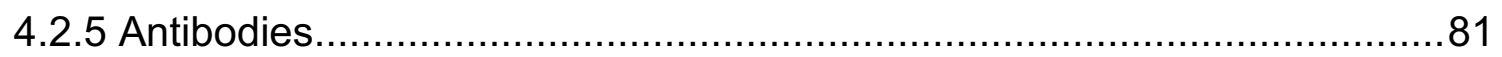

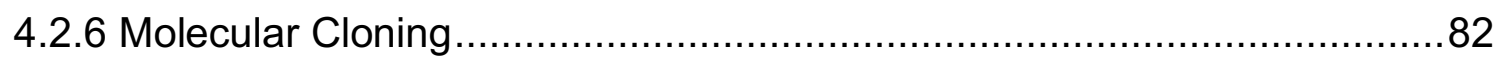

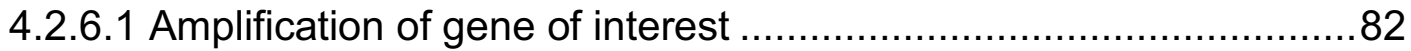

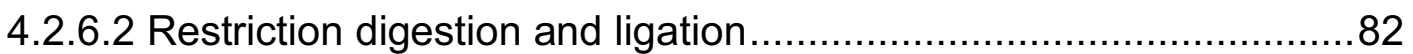

4.2.6.3 Transformation of chemically competent $E$. coli ..........................83

4.2.6.4 Plasmid DNA extraction and sequencing ................................... 83

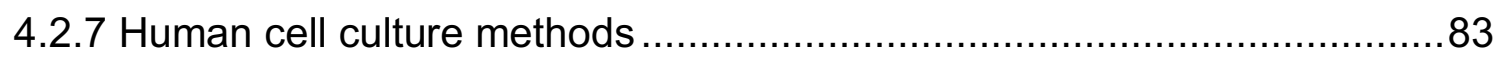

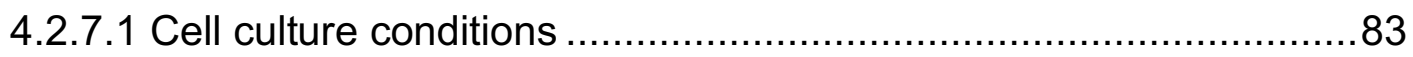

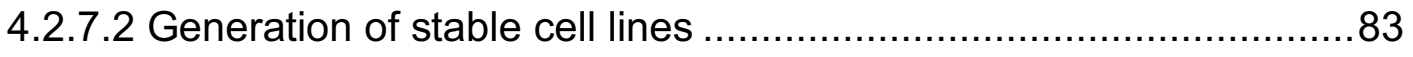

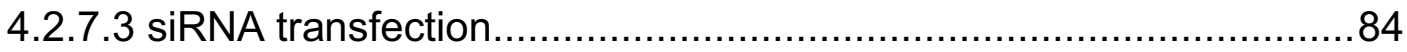

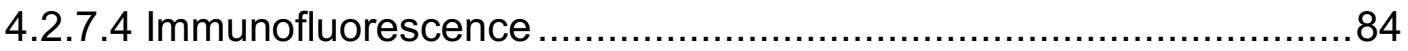

4.2.7.5 Analysis of pre-ribosomal complexes by sucrose density

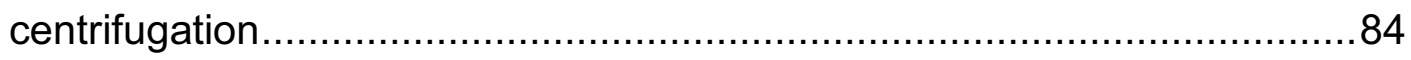

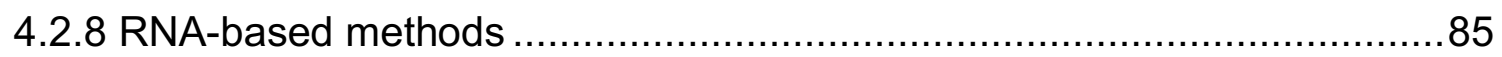

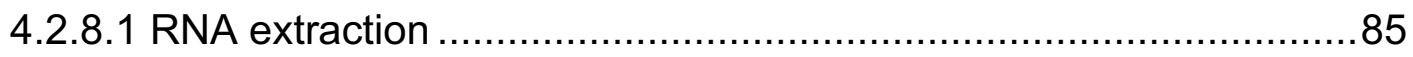

4.2.8.2 Pulse chase metabolic labelling of newly synthesised RNA and

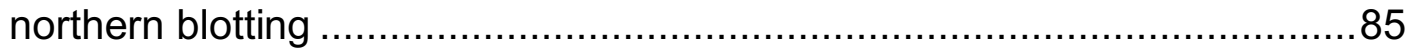

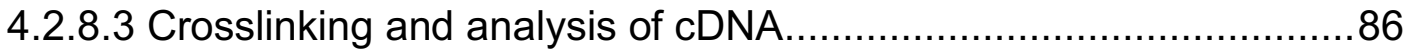

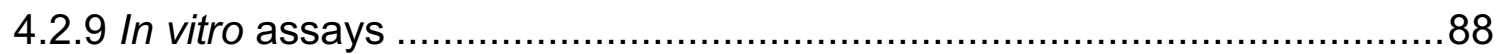

4.2.9.1 Recombinant protein expression and purification.........................88

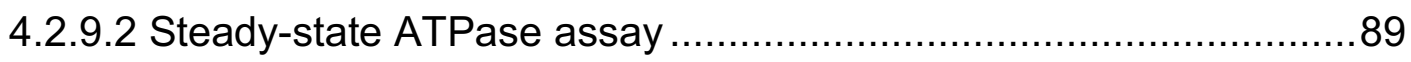

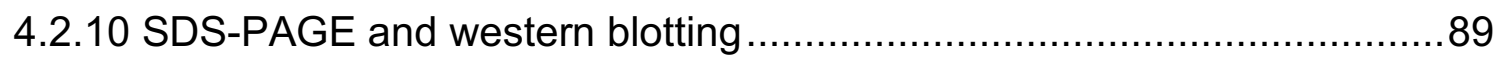

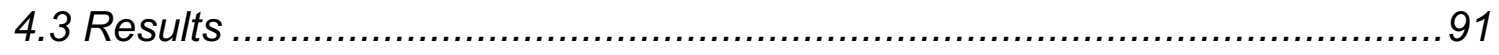

4.3.1 DDX55 is an ATPase and a mutation in its conserved GKT motif inhibits its

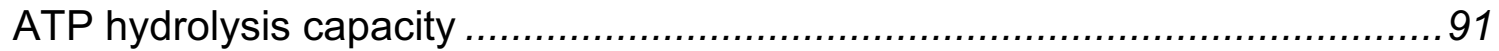

4.3.2 The human helicase DDX55 is required for ribosome biogenesis ...........92

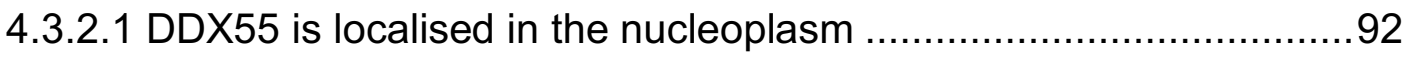

4.3.2.2 Depletion of DDX55 in vivo impairs ribosome biogenesis ..............93

4.3.2.3 DDX55 crosslinks to helices 62 and 63 of the $28 \mathrm{~S}$ rRNA sequence in vivo 
4.3.3 C-terminal tail of DDX55 is required for its recruitment to pre-ribosomal subunits .99

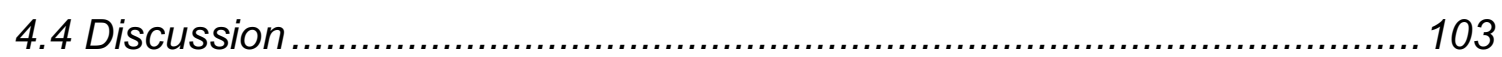

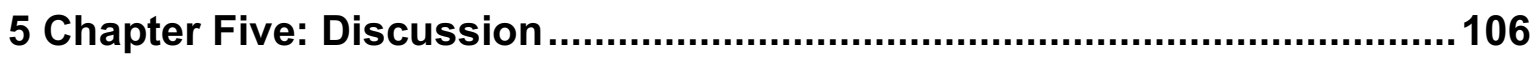

5.1 Distinct roles of RNA helicase in ribosome biogenesis.............................106

5.2 Division of labour: DEAH-box versus DEAD-box helicases.......................108

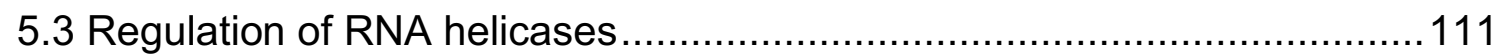

5.4 Dynamics of RNA helicase association with pre-ribosomes....................113

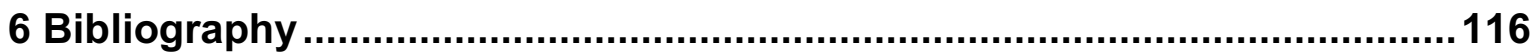

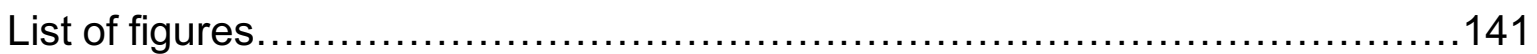

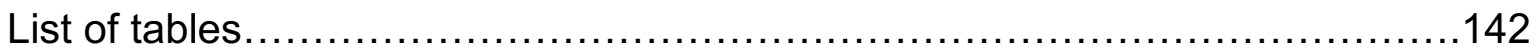

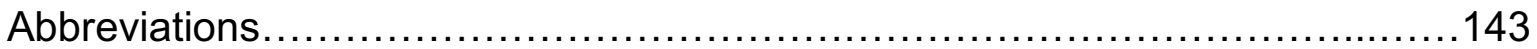

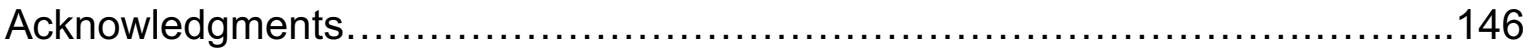

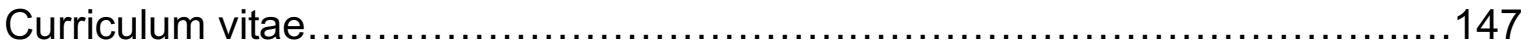




\begin{abstract}
Eukaryotic ribosomes are essential ribonucleoprotein (RNP) complexes consisting of four ribosomal RNAs (18S, 5S, 5.8S, 28S/25S) and approximately 80 ribosomal proteins, which are required for the process of translating the information encoded in mRNAs into proteins. Ribosome biogenesis requires a dynamic interplay between a multitude of trans-acting biogenesis factors such as ATPases, GTPases, snoRNAs, nucleases and RNA helicases, which assist in the maturation of pre-ribosomal subunits. RNA helicases are RNAdependent NTPases that play key roles in all aspects of RNA metabolism including premRNA splicing, translation, transcription and ribosome biogenesis. The majority of RNA helicases involved in ribosome biogenesis belong to either the DEAD- or DEAH-box family and consist of a structurally conserved helicase core flanked by $\mathrm{N}$ - and $\mathrm{C}$-terminal domains that can serve as platforms for protein-protein/protein-RNA interaction. In yeast, 21 RNA helicases are implicated in the process of ribosome biogenesis and likely play key roles in the structural remodelling of pre-ribosomal subunits. Genome-wide screens and proteomic analyses of mammalian nucleoli have suggested the involvement of many orthologues of yeast RNA helicases in the human ribosome assembly pathway as well as highlighting additional helicases that may regulate the process in humans.

Although several RNA helicases are implicated in human ribosome assembly, the precise physiological roles and in vivo substrates of these helicases are often not wellcharacterized. In this study, we aimed to gain insights into the roles of two RNA helicasesDHX37 and DDX55, which are implicated in SSU and LSU biogenesis respectively.

An RNAi-based rescue system indicated that the catalytic activity of DHX37 is required for the effective maturation of 18S rRNA. Using an in vivo crosslinking approach, we show that DHX37 crosslinks to the U3 snoRNA and that expression of a catalytically inactive mutant of DHX37 leads to the accumulation the pre-ribosomal accumulation of U3, suggesting a role for DHX37 in release of the U3 snoRNP from pre-ribosomal particles. In addition, we identified UTP14A as a cofactor of DHX37, and our data suggest that UTP14A and DHX37 act in a synergistic manner to promote the dissociation of U3 snoRNA from pre-ribosomal subunits. While our data implied that DHX37 mediated release of U3 snoRNP is conserved in eukaryotes, we also revealed several interesting differences between the yeast and human proteins in terms of recruitment and function of the helicase during late steps of ribosome assembly. Furthermore, our crosslinking data also identified a binding site of DDX55 on the 28S rRNA sequence, consistent with its requirement for LSU maturation. DDX55 was found to predominantly localize in the nucleoplasm suggesting a role in latestage ribosome biogenesis. We further investigated the function of the lysine-rich C-terminal tail of DDX55 and our results indicate a possible role of the C-terminal tail in the recruitment
\end{abstract}


of DDX55 to pre-ribosomal complexes. Taken together, our results provide important new insights into the roles of RNA helicases in human ribosome biogenesis. 


\section{Chapter One: Introduction}

\subsection{Overview of ribosome assembly pathway}

Translation of the nucleotide sequences of mRNAs into the amino acid sequences of proteins is an essential cellular process that is carried out by large macromolecular complexes called ribosomes. Eukaryotic cytoplasmic $80 \mathrm{~S}$ ribosomes consist of two ribonucleoprotein (RNP) complexes: the large subunit (LSU; 60S), consisting of three ribosomal RNAs (rRNA; 28S (humans)/25S (yeast), 5.8S, 5S) along with 47 (humans) /46 (yeast) ribosomal proteins (RPs) and the small subunit (SSU; 40S), comprising the $18 \mathrm{~S}$ rRNA along with 33 proteins (Anger et al., 2013; Ben-Shem et al., 2011). The synthesis of a macromolecular complex of such complexity is one of the most energetically demanding processes in eukaryotic cells and requires the assistance of over 200 ribosome assembly factors in yeast (Woolford and Baserga, 2013).

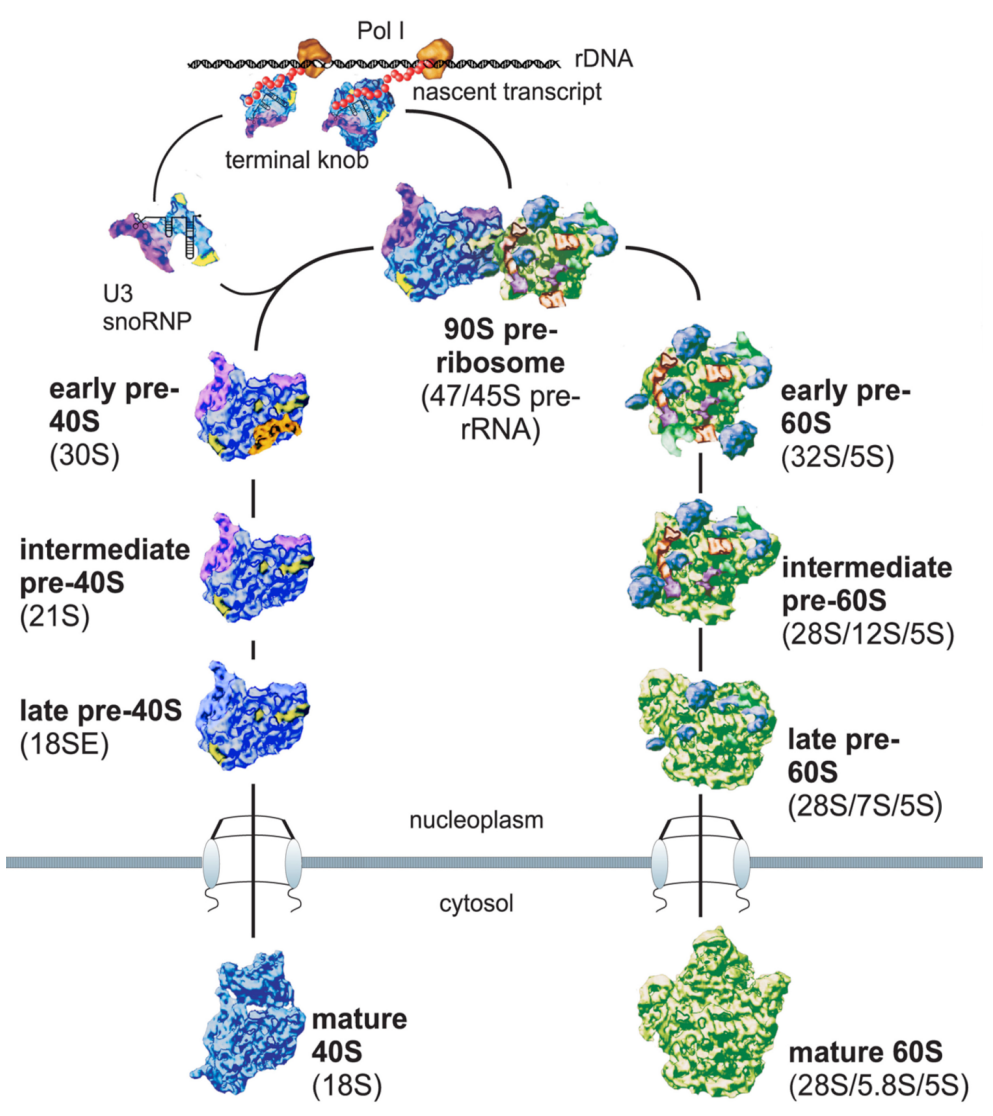

Figure 1.1. Ribosome biogenesis in humans. Ribosome assembly initiates in the nucleolus with the transcription of $47 \mathrm{~S}$ nascent transcript by RNA polymerase I. The nascent transcript harbours the sequences of mature rRNAs (18S, 5.8S, 28S) along with internal (ITS1 and ITS2) and external (5'ETS and $3^{\prime} E T S$ ) spacer sequences. Several factors associate co-transcriptionally to generate the $90 \mathrm{~S}$ preribosome complex. An endonuclease mediated cleavage separates the maturation pathways for the two subunits. The pre-60S and pre-40S undergo independent maturation steps assisted by over 200 transacting factors before export into the cytoplasm (Panel adapted from Martin, 2014). 
The pre-ribosomal subunits undergo extensive nucleolytic processing and structural transitions assisted by a variety of enzymes including AAA-ATPases, helicases, kinases and nucleases (Figure 1.1) (Bohnsack and Bohnsack, 2019; Klinge and Woolford, 2019; Woolford and Baserga, 2013). The pre-ribosomal subunits are exported out of the nucleus to the cytoplasm where the final steps of maturation occur (Sloan et al., 2016). Although ribosome biogenesis is a highly conserved process, best studied in Saccharomyces cerevisiae (yeast), there are significant differences in terms of pre-rRNA processing and the functions of ribosomal assembly factors in higher eukaryotes.

\subsubsection{Ribosome biogenesis in yeast and humans}

In yeast, RNA polymerase I mediated transcription leads to the generation of the $35 \mathrm{~S}$ prerRNA consisting of internal and external spacer sequences (ITS1 and ITS2, and 5' ETS and 3' ETS) along with the sequences of 18S, 5.8S, 25S rRNAs (Long and Dawid, 1980). The 5S rRNA is transcribed in the opposite direction by RNA polymerase III (Figure 1.2) (Sentenac, 1985).

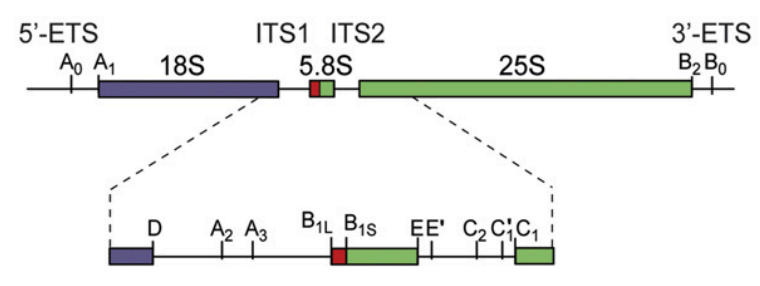

Figure 1.2. Schematic representation of yeast 35S pre-rRNA transcript. The nascent 35S prerRNA contains the sequence of mature $5.8 S, 25 S$ and 18S rRNA (rectangles) as well as the sequences of external and internal transcribed spacers (adapted from Mullineux and Lafontaine, 2012).

The nascent $35 \mathrm{~S}$ pre-rRNA transcript undergoes extensive rearrangement mediated by different classes of factors including small nucleolar ribonucleoprotein complexes (snoRNPs), RNA helicases and energy consuming enzymes such as GTPases and AAAATPases (Henras et al., 2008; Woolford and Baserga, 2013). A series of highly coordinated, hierarchical processes give rise to the earliest nascent pre-ribosomes known as $90 S$ pre-ribosomes, comprising a large RNP complex, the SSU processome. Although the composition of the $90 \mathrm{~S}$ and the SSU processome differ slightly, both complexes consist primarily of small subunit proteins and assembly factors (Phipps et al., 2011). Subsequent RNA cleavage events separate the sequences of the mature rRNAs from the spacer segments (Venema and Tollervey, 1995). Cleavage at site $A_{2}$, within ITS1 results in the separation of the maturation pathways for the SSU and LSU (Udem and Warner, 1972). Although co-transcriptional cleavage at site $A_{2}$ is most prevalent (occuring in $2 / 3^{\text {rd }}$ of the cases), it is not obligatory. Post-transcriptional processing by the RNase mitochondrial RNA processing (MRP) at the $A_{3}$ site also results in the separation of the two pre-ribosomal complexes (Lygerou et al., 1996). UTP24 mediated cleavages at the $A_{1}$ and $A_{2}$ sites lead to 
the generation of $20 \mathrm{~S}$ and $27 \mathrm{SA}_{2}$ pre-rRNAs. $20 \mathrm{~S}$ pre-rRNA is finally processed to $18 \mathrm{~S}$ rRNA by Nob1 (Pertschy et al., 2009; Wells et al., 2016). Various cryogenic electron microscopy (cryo-EM) structures available for the SSU processome provide molecular snapshots of the earliest stable precursor (Barandun et al., 2017). Interestingly, as the structures obtained by cryo-EM were remarkably similar even when captured under variable physiological conditions, the SSU processome appears to be a very stable intermediate that is formed during the early events of ribosome biogenesis (Barandun et al., 2017; Cheng et al., 2017; Sun et al., 2017). The processing of the LSU precursor $27 \mathrm{SA}_{2}$ can occur via two alternative pathways, ultimately leading to the generation of $25 \mathrm{~S}$ rRNA and $6 \mathrm{~S}$ pre-rRNA. Lastly, after the release of early factors, export adaptors promote the export of pre-60S particles into the cytoplasm wherein further processing of the $6 \mathrm{~S}$ pre-rRNA sequence occurs to form the mature $5.8 \mathrm{~S}$ rRNA. Assembly factors also dissociate from pre-60S driven by the action of AAA-ATPase Drg1 leading to the maturation of the large subunit (Lo et al., 2010; Pertschy et al., 2007)

As the sequences of the mature rRNAs and the structure of ribosome is evolutionary conserved, there has been a long-standing assumption that the process of ribosome biogenesis is generally conserved throughout eukaryotes. Moreover, most assembly factors that have been first described in yeast, possess functional homologues in humans (Wild et al., 2010). However, in the recent years, there is compelling evidence suggesting that the process of ribosome biogenesis has acquired greater complexity in humans due to the extension of the primary pre-rRNA transcript (13.3 kb in human vs $6.7 \mathrm{~kb}$ in yeast) and emergence of metazoan-specific cleavage sites (Lazdins et al., 1997). Proteomic analysis of the mammalian nucleoli revealed an inventory of 4500 putative (Ahmad et al., 2009) nucleolar proteins in contrast to 150 nucleolar proteins found in budding yeast (Huh et al., 2003). RNAi-mediated screens monitoring pre-rRNA processing defects upon effective depletion identified 286 pre-rRNA processing factors, including 74 lacking yeast homologues (Tafforeau et al., 2013). Furthermore, another genome-wide screen examining nucleolar morphology or number as readouts identified 139 hits consisting of both nucleolar (27.3\%) and non-nucleolar proteins $(72.7 \%)$ revealing the involvement of proteins outside the nucleolus as putative regulators of ribosome biogenesis (Farley-Barnes et al., 2018). Detailed study and characterisation of some of these assembly factors highlight interesting differences between ribosome biogenesis in yeast and humans. For instance, a microscopy-based screen monitoring the localisation of pre-ribosomal subunits highlighted important differences in the assembly pathway of the $60 \mathrm{~S}$ ribosomal subunit. Similar to yeast, the exportin CRM1 along with adaptor proteins such as NMD3 are implicated in the export of pre-60S subunits in humans (Güttler and Görlich, 2011). Additionally, Exportin 5 (Exp5) is implicated in pre-60S export into the cytoplasm and its depletion leads to 
significant defects in LSU maturation (Wild et al., 2010). In contrast, deletion of Msn5 (homologue of Exp5 in yeast) does not affect ribosomal subunit maturation indicating that the pathways for pre-60S export are not conserved between yeast and humans (StageZimmermann et al., 2000). These screens confirm the notion that although many facets of ribosome biogenesis are conserved from yeast to humans, there is a higher degree of complexity in humans as revealed by the involvement of several metazoan-specific assembly factors and the precise roles of many of these factors have still not been elucidated.

\subsection{2 pre-rRNA maturation in humans}

In humans, nascent $47 \mathrm{~S}$ pre-rRNA (containing 18S, 5.8S, $28 \mathrm{~S}$ rRNA sequences) is synthesized by RNA polymerase I from repeats of rDNA sequences situated on chromosomes 13,14,15, 21 and 22 . The 5 S rRNA is synthesized by RNA polymerase III from multiple genes located on chromosome 1, found in close proximity to the nucleoli (Fedoriw et al., 2012). Within the primary transcript, the sequences of the mature rRNAs are flanked by external and internal transcribed spacer sequences (ITS1 and ITS2, and 5' ETS and 3' ETS), which are excised by the sequential and co-ordinated action of endo- and exo-nucleases (Figure 1.3). In mammalian cells, the 47S pre-rRNA is initially processed at site $A^{\prime}$ and 02 to generate the $45 S$ pre-rRNA. Interestingly, the A' cleavage site is only found in metazoans (Lazdins et al., 1997). As cleavage at this site did not play a role in downstream processing events (Sloan et al., 2014), the functional relevance of this additional cleavage site still needs to be elucidated. However, as human ribosome biogenesis has a higher degree of complexity compared to yeast, the emergence of an additional cleavage site has been suggested to play a role in quality control during the early stages of ribosomal subunit maturation (Sloan et al., 2014; Wang and Pestov, 2011). Cleavage of the 45S pre-rRNA can either initiate in 5'ETS or within the ITS1 region. The co-existence of alternate pathways for pre-rRNA processing may lead to changes in the ratios of precursors based on cell type or stress conditions (Choesmel et al., 2006; Flygare et al., 2006) and suggests a mechanism for fine-tuning the kinetics and pattern of pre-rRNA processing in higher eukaryotes (Henras et al., 2015). In the main pre-rRNA processing pathway, initial cleavage at site 2 within the ITS1 region performed by RNase MRP results in the generation of $30 \mathrm{~S}$ and $32.5 \mathrm{~S}$ rRNA precursors. The $5^{\prime}$ end of $30 \mathrm{~S}$ pre-rRNA is processed by co-ordinated cleavages at site $A_{0}$ and 1 and the endonuclease hUTP24 is suggested to be required for these events to occur (Tomecki et al., 2015; Wells et al., 2016). The removal of 5' ETS from 30S results in the formation of the 21S pre-rRNA, which gets further processed at the 3' end by the action of RRP6 (Preti et al., 2013; Sloan et al., 2013a; Tafforeau et al., 2013) leading to the generation of the 21S-C pre-rRNA species. A 
subsequent cleavage at site $E$ results in the generation of $18 \mathrm{~S}-\mathrm{E}$, which is first processed by poly-A-specific ribonuclease (PARN) in the nucleus before it is exported to the cytoplasm and undergoes a final cleavage by the endonuclease NOB1 to generate the mature $18 \mathrm{~S}$ rRNA (Bai et al., 2016; Montellese et al., 2017; Preti et al., 2013; Sloan et al., 2013a).

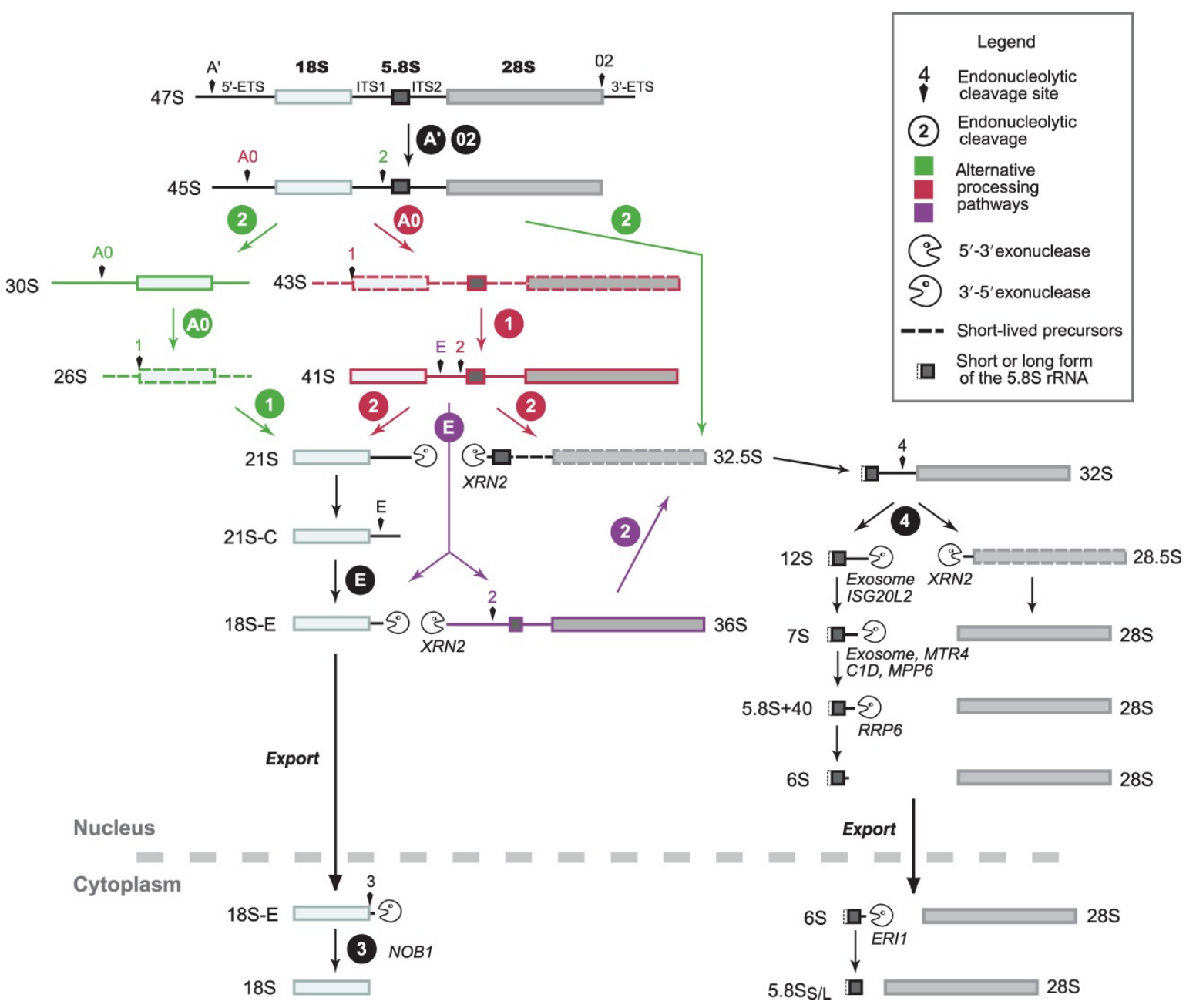

Figure 1.3. Overview of pre-rRNA processing in humans. The 47S rRNA synthesized by RNA polymerase $I$ is cleaved at site $A^{\prime}$ and 02 to generate the $45 S$ rRNA. Cleavage can then occur either within the ITS1 region (green, major pathway) or the $5^{\prime} \mathrm{ETS}$ region (red, minor pathway). If cleavage within the 5'ETS occurs first, $41 \mathrm{~S}$ pre-rRNA is generated which can be either cleaved at site $E$ (purple) or site 2 . The alternate pathways lead to the production of $21 \mathrm{~S}-\mathrm{C}$ and $32 \mathrm{~S}$, the common precursors of small and large subunit rRNAs. After export of the pre-ribosomal subunits into the cytoplasm, the pre-rRNAs undergo final processing steps to form the mature sequences of 18S, 5.8S and 28S (Henras et al., 2015).

Alternatively, the 45S pre-rRNA can be first cleaved within the 5'ETS region leading to the generation of the $41 \mathrm{~S}$ pre-rRNA. This intermediate can be processed through two different pathways. In the major pathway, cleavage at site 2 within the ITS1 sequence leads to the generation of $21 \mathrm{~S}$ and $32.5 \mathrm{~S}$ pre-rRNAs. However, cleavage at site $\mathrm{E}$ (located upstream of site 2) results in the formation of 18S-E and 36S precursors (Preti et al., 2013; Sloan et al., 2013a). The 36 S pre-rRNA is further processed at the 5' end by the 5' - 3' exonuclease 
XRN2 to produce $32.5 S$ pre-rRNA, a short-lived precursor which is rapidly converted to the $32 S$ pre-RNA, which is an abundant, common LSU precursor arising out of all alternative processing pathways. The $32 \mathrm{~S}$ pre-rRNA is cleaved within the ITS2 region to form the $12 \mathrm{~S}$ and 28.5S pre-rRNAs (Schillewaert et al., 2012). 28.5S pre-rRNA is processed by XRN2 to produce the mature $28 \mathrm{~S}$ rRNA. The $12 \mathrm{~S}$ pre-rRNA is processed further by numerous 3' - 5' exonucleases to generate the 7S pre-rRNA (Couté et al., 2008; Michot et al., 1999) which subsequently gets processed by the nuclear exosome to form $6 \mathrm{~S}$ pre-rRNA (Tafforeau et al., 2013), which is exported to the cytoplasm as part of the pre-LSU complex. The final 3' trimming of $6 \mathrm{~S}$ pre-rRNA by exonuclease ERI1, results in the formation of mature $5.8 \mathrm{~S}$ rRNA (Ansel et al., 2008).

Along with nuclease-mediated processing of the pre-rRNA transcript, nucleotide modifications of pre-rRNA sequences (Littlefield and Dunn, 1958a, 1958b; Smith et al., 1992) play a significant role in the maturation of pre-rRNAs. Most modifications are wellconserved amongst species and are found to be clustered around functionally important regions of ribosomes (Decatur and Fournier, 2002). The most common rRNA modifications are either methylation of the 2'-hydroxl group of the sugar residue mostly catalysed by the box C/D snoRNPs or the conversion of uridine residues to pseudouridine mediated by box H/ACA snoRNPs (Decatur and Fournier, 2002). C/D box snoRNPs have also been implicated in the acetylation of cytosine residues in yeast and humans (Sharma et al., 2015). Each snoRNP complex combines a set of proteins, responsible for catalysing the enzymatic reaction along with a specific guide RNA, which base-pairs with the pre-rRNA target region. Apart from these, few modifications are catalysed by specific stand-alone enzymes (Henras et al., 2017; Sloan et al., 2017). As extensive base-pairing with target pre-rRNAs is a prerequisite for snoRNPs to act, most modifications are thought to be installed in the early stages of ribosome biogenesis when the pre-ribosome structure is more open. In human rRNAs, currently 228 modified sites have been identified as opposed to 112 in the yeast rRNAs, and the higher density of rRNA modifications has been attributed to the fact that human ribosome biogenesis is more complex and hence, needs additional regulation via rRNA modifications (Sloan et al., 2017). Although the functional relevance of many of these modified sites remains unknown, it has been suggested that modified nucleotides alter the chemical and topological properties of the basic nucleotides and can therefore, act to structurally stabilize the ribosomal RNAs, thereby influencing translation (King et al., 2003; Yoon, 2006).

Apart from nucleotide modifications, several snoRNAs - including U3 (SNORD3), U14A/B (SNORD14A/B) and the metazoan-specific snoRNAs U8 (SNORD118) and U22 (SNORD22)- play an important role in mediating pre-rRNA folding and cleavage (Watkins and Bohnsack, 2012). Base-pairing between distinct distant regions of pre-rRNAs and the 
corresponding snoRNAs results in the interlocking of pre-rRNA, thereby promoting its folding and subsequent processing. For example, long range interactions formed between the U3 snoRNA and the 18S rRNA sequence in the early stages of small subunit biogenesis are important for the efficient maturation of the 18S rRNA. Moreover, the timely release of U3 snoRNA is critical for the maturation of the small subunit (Sardana et al., 2015). Similarly, it was demonstrated in humans that snoRNA guided pre-rRNA folding can influence important cleavage events in the final steps of SSU assembly. For example, the endonuclease NOB1, responsible for the final step of 18S rRNA maturation, initially is recruited away from its cleavage site. However, structural rearrangements during final steps of pre-40S biogenesis bring NOB1 into close proximity to its site of action, triggering the formation of mature 18S rRNA (Ameismeier et al., 2018; Larburu et al., 2016).

\subsubsection{Role of ribosomal proteins}

Ribosomal proteins (RPs) play crucial roles in the assembly of eukaryotic ribosomes in vivo and mutations in genes encoding RPs are associated with a wide range of ribosomeassociated diseases, especially bone marrow failures (Liu and Ellis, 2006) . The expression of all 80 RPs in humans is precisely regulated and co-ordinated to maintain equal levels in order to ensure appropriate availability for subunit assembly. Hence, most genes encoding RPs share a common promotor sequence. Structural analyses of mature ribosomes have revealed that RPs generally comprise globular domains interspersed by unstructured loops responsible for binding to rRNA, to other RPs or trans-acting assembly factors (Melnikov et al., 2012). Upon translation in the cytoplasm, chaperones bind co-translationally to nascent RPs facilitating their import into the nucleus wherein they associate intimately with preribosomal particles (de la Cruz et al., 2015; Pillet et al., 2017). Although precise roles of many RPs during ribosome biogenesis are not known, it is thought that RPs can also act as chaperones during ribosome assembly that assist in processing and proper folding of nascent pre-rRNAs (Robledo et al., 2008). Furthermore, it has been demonstrated that RNAi-mediated depletion of RPs of the SSU impairs maturation of the 18S rRNA (O'Donohue et al., 2010). Depletion of LSU RPs results in the impaired production of $28 \mathrm{~S}$ and 5.8S rRNA and thus, the decreased production of $60 S$ ribosomal subunit. RPL5 is involved in the processing of pre-5S rRNA and forms the RPL5/5S complex that undergoes nuclear import. In the nucleolus, the RPL5/5S complex associates with RPL11 to form the 5S RNP complex which gets incorporated into the pre-60S subunit (Ciganda and Williams, 2011; Sloan et al., 2013b; Steitz et al., 1988). For the maturation of the SSU, a subset of RPs have been shown to associate with 905 pre-ribosomal complexes and play a role in the early stages of ribosome assembly. The assembly of the first set of RPs helps to attain a critical conformational state before further processing can occur. This is followed by the 
assembly of RPs that initiate the folding of the 'head' domain. The second set of RPs, which are incorporated later, are important for efficient processing of ITS1 and structuring the 3' domain of 18S rRNA (O'Donohue et al., 2010). The sequential assembly of RPs leads to an ordered 5' - 3' structuring of the human 405 and was similar to what was observed in yeast (Ferreira-Cerca et al., 2005) and in prokaryotes (Chen and Williamson, 2013) suggesting that this is a highly conserved, critical mechanism for ensuring fidelity of the maturation pathway. Although there is considerable conservation of RPs between yeast and humans, various studies focussing on human ribosome biogenesis have highlighted differences. For example, in humans, knockdown of small subunit proteins, RPS7 and RPS28 impairs processing of 5'ETS and ITS1 (Robledo et al., 2008). In contrast, yeast Rps7 and Rps28 affect the cytoplasmic maturation steps of the 18S rRNA and the pre-40S subunit (Ferreira-Cerca et al., 2005, 2007). Furthermore, depletion of RPS15 in humans, allows efficient export of $18 \mathrm{~S}-\mathrm{E}$ pre-rRNA to the cytoplasm, but final cytoplasmic maturation steps of pre-40S are affected (Robledo et al., 2008). However, in yeast, Rps15 was observed to be crucial for the export of the 20S pre-rRNA (analogous to human 18S-E) from the nucleus (Léger-Silvestre et al., 2004).

In addition to their roles in the assembly pathway of ribosomal subunits, several ribosomal proteins have been implicated in carrying out extra-ribosomal activities. For example, RPs have been shown to play important roles in the innate immune response. Extra-ribosomal RPL13A can act as a translational regulator and can modulate the expression of Interferon$\gamma$ (Mukhopadhyay et al., 2009). Additionally, RPL22 and RPL22L1 have been reported to play a key role in the modulation of splicing of distinct pre-mRNAs (Zhang et al., 2017) and RPS13 and RPL10a are implicated in the process of alternative splicing (Malygin et al., 2007; Takei et al., 2016). Another prominent example of an extra-ribosomal function of RPs is the 5S RNP complex (Pelava et al., 2016). The tumour suppressor, p53 is regulated by the levels of the E3 ubiquitin ligase HDM2. HDM2 together with its related protein HDMX binds p53, thereby inhibiting its transcriptional activity and marking the protein, via ubiquitination, for subsequent degradation. In case of defective ribosome assembly, unincorporated, nucleoplasmic 5S RNP complex binds HDM2, blocking its action and leads to the stabilization of p53 (Donati et al., 2013; Sloan et al., 2013b). The 5S-HDM2 pathway has now been shown to act as the sensor responsible for lipid homeostasis (Oie et al., 2014), nutrient stress and oxidative stress (Liu et al., 2016; Pelava et al., 2016).

\subsubsection{Structural transitions during subunit assembly in humans}

Apart from RPs, formation of mature, functional ribosomal subunits is also assisted by a variety of trans-acting ribosome biogenesis factors (RBFs) which co-ordinate proper folding and processing of pre-rRNAs. Unlike RPs, RBFs are not part of the mature ribosome 
subunits but engage relatively transiently to ensure directionality, sequential assembly of RPs and productive formation of stable pre-rRNA folding intermediates. Major classes of RBFs involved in ribosome assembly include nucleases responsible for pre-rRNA processing and removal of spacer sequences, modification enzymes that carry out nucleotide modifications, GTPases, which act as important placeholders and molecular switches, ATPases that are essential for mediating conformational rearrangements and nuclear export factors. Importantly, RNA helicases act as crucial remodellers of preribosomal complexes by regulating RNA-RNA or RNA-protein interactions at specific stages of subunit maturation (discussed in detail in section 1.2).

Due to recent advancements in structural biology techniques such as cryo-EM, snapshots of various stages (from middle to late) of human $40 \mathrm{~S}$ subunit are now available, highlighting the vast range of structural rearrangements that take place resulting in the maturation of the small ribosomal subunit (Ameismeier et al., 2018; Larburu et al., 2016). Assembly of the SSU occurs in sequential, discrete steps involving a large interaction network of RBFs. The maturation of the $40 \mathrm{~S}$ 'body' domain is completed by the recruitment of several RPs, leading to the recruitment of the nuclease NOB1. At the same time, export adaptor RRP12 binds to the 'head' region of the pre-40S subunit containing the 3' major domain of the 18S rRNA and mediates essential structural rearrangements of the pre-40S complex. The release of RRP12 in cytoplasm is followed by the recruitment of the translational initiation factor, RACK1. Interestingly, in yeast, RACK1 is not present in pre-40S complexes (Larburu et al., 2016) and Rrp12 interacts with nucleoporins to aid the export of pre-40S particles (Oeffinger et al., 2004). In humans, the release of RRP12 has been suggested to ensure the fidelity of the assembly pathway by coupling correct 'head' formation and nuclear export. The biogenesis factors ENP1 and LTV1 are present in the 'beak' region of late pre-40S particles, preventing the folding of rRNAs into their mature conformations and the binding of specific RPs. LTV1 and ENP1 are phosphorylated by the kinase CK1 $\delta / \varepsilon$, which triggers their dissociation from the pre-40S particles (Ghalei et al., 2015; Zemp et al., 2014) leading to the stable incorporation of RPS3 and the proper arrangement of the rRNA helices that form the mature 'beak' structure. Maturation of the decoding centre, which harbours the $A$ and the $P$ sites within the pre-40S subunit, entails the precise folding between h44 and h28 or h45. The kinase RIOK2, along with the inactive GTPase TSR1 and LTV1, bind and stabilize h44 maintaining the helices in immature conformational states and blocking proper folding of this region (Ameismeier et al., 2018; Larburu et al., 2016). Along with these RBFs, the RNA-binding protein PNO1 stabilizes h28 and h45 in an immature state and also helps spatially retain NOB1 distant from its target cleavage site. Release of PNO1 results in a large structural rearrangement, promoting the rearrangement of the 3 ' end of 18S rRNA into the catalytic pocket of NOB1, leading to its cleavage. In yeast, Dim1 is responsible for 
methylation of two adenosine residues (A1781/A1782) in the 18S rRNA in the cytoplasm (Lafontaine et al., 1998). Interestingly, in humans, DIMT1L carries out the equivalent methylations in the 18S rRNA sequence in the nucleus and is not exported out into the cytoplasm along with the pre-40S subunit (Zorbas et al., 2015). Although the purpose of this difference in spatial compartmentalization is not yet understood, it has been suggested that the methylation status of the 18S rRNA can serve as an important quality-control checkpoint and commit the pre-40S subunits for nuclear export (Zorbas et al., 2015). Export of pre-40S particles into the cytoplasm is mediated by the exportin CRM1 in association with adaptor proteins such as RIOK2 and PDCD2L (Landry-Voyer et al., 2016). The nuclear transport factors Mex67-Mtr2 play crucial roles in the export of pre-40S and pre-60S subunits in yeast (Faza et al., 2012), however, their human homologues were not found to be required for pre-40S export (Wild et al., 2010), suggesting that this function of these proteins might not be conserved in higher eukaryotes.

While in yeast structural information on several pre-60S particles is already available, less is currently known about the structural rearrangements that occur during 605 biogenesis in humans. In yeast, the AAA-ATPase Rea1 plays a key role in triggering the release of ribosomal proteins during the maturation of $60 \mathrm{~S}$ particles. The orthologue of Rea1 in humans, MDN1, has been suggested to play a similar role (Raman et al., 2016). One aspect of pre-60S maturation that has been well-studied in humans is the release of pre-60S factor elF6 (Tif6 in yeast). Tif6 associates with pre-60S subunits in yeast and acts as antiassociation factor by inhibiting the association of pre-60S and (pre-) 40S subunits in the cytoplasm and is released by action of the GTPase, Efl1 (Senger et al., 2001). In humans, elF6 is reported to bind to ribosomal proteins RPL3, RPL14 and RPL24 and prevents the joining of subunits by inhibiting inter-subunit bridge formation (Gartmann et al., 2010). This event seems to be conserved as recruitment of the GTPase EFL1 leads to the release of elF6 (Gartmann et al., 2010; Klinge et al., 2011). 


\subsubsection{Ribosomopathies}

Ribosomopathies constitute a growing class of diseases caused by mutations in genes encoding RPs and RBFs. These abnormalities can alter the quantity of ribosomes by preventing the production of translation efficient subunits or affect the quality by altering the protein composition or modification pattern (Danilova and Gazda, 2015; Mills and Green, 2017; Narla and Ebert, 2010). Ribosomopathies are often associated with developmental abnormalities and increased risk of cancer. Diamond-Blackfan anemia (DBA) is the most well-understood of all the ribosomopathies with its link to ribosome biogenesis clearly established. DBA is relatively rare with 5-7 cases per million live births. Mutation in 19 RP genes have now been associated with DBA, which is characterized by bone marrow failure leading to severe anaemia (Da Costa et al., 2018). Mutations in the RP genes resulting in DBA include missense, nonsense or frameshift mutations resulting in impaired mRNA synthesis or stability leading to decreased RP production (Boria et al., 2010). Several other congenital disorders resulting in haematopoietic dysregulation are also associated with mutations in genes encoding RBFs, including Shwachman-Diamond syndrome (Shwachman et al., 1964), 5q- syndrome (Trainor et al., 2009) and Dyskeratosis congenita (Knight et al., 2001). Additionally, both Treacher-Colins syndrome (Vincent et al., 2016) and Bowen-Conradi syndrome (Armistead et al., 2009), which result in craniofacial defects, are caused by mutations in RBFs. Affecting ribosome biogenesis, thus often leads to overlapping phenotypic characteristics; therefore, understanding the tissue-specific defects that manifest as a result of impaired ribosome synthesis still remains a challenge. Several models have been suggested in order to explain the molecular basis of ribosomopathies. The number of ribosomes is tightly controlled in cells, a phenomenon known as ribosome homeostasis. Ribosome deficiency in cells, as a result of mutations in genes encoding RPs and assembly factors, might lead to changes in ribosome homeostasis. As the number and concentration of ribosomes can vary depending on cell type and cell-cycle phase, alterations in the number of functional ribosomes can adversely impact the synthesis of specific proliferation regulators. This leads to the tissue-specific pathologies as observed in ribosomopathies. In the case of DBA, genes involved in erythroid differentiation are observed to be most sensitive to the reduced number of ribosomes (Khajuria et al., 2018; Ludwig et al., 2014). Additionally, new techniques that enable detailed investigations into the stoichiometry of rRNA modifications and the protein composition of ribosomes, suggest that ribosomes can have varied modifications and may differ in their protein compositions. These differences imply the presence of heterogeneous populations of ribosomes (Bortoluzzi et al., 2001; Simsek et al., 2017). Hence specialized ribosomes might be important for the translation of specific mRNAs that are involved in development and proliferation. Therefore, alterations in ribosome composition due to mutation in genes 
encoding RPs or RBFs might affect tissue specific pathways. Moreover, impaired ribosome biogenesis can also lead to the accumulation of the 5S RNP complex that can bind to the HDM2 ubiquitin ligase and sequester it, resulting in the stabilization of p53 leading to cell cycle arrest and apoptosis (Donati et al., 2013; Sloan et al., 2013b). Many of the developmental defects in ribosomopathies can be attributed to the activation of p53. Moreover, prolonged stimulation of p53 also leads to loss of sensitivity resulting in higher susceptibility to cancer as observed in many ribosomopathies.

\subsection{RNA helicases}

RNA helicases are ubiquitously expressed and highly conserved enzymes that participate in nearly all aspects of RNA metabolism. These proteins bind and remodel RNA or RNPs in a nucleoside triphosphate (NTP) dependent manner (Tanner et al., 2001). RNA helicases comprise a structurally conserved core, often flanked by $\mathrm{N}$ - and $\mathrm{C}$-terminal domains that contribute to their specificity. Although RNA helicases were originally defined as enzymes that play a key role in unwinding RNA duplexes, they are now known to be involved in a wide range of molecular remodelling functions such as strand annealing, displacement of protein from RNA and acting as RNA clamps (Bourgeois et al., 2016).

\subsubsection{RNA helicase superfamilies and conserved sequence motifs}

DNA and RNA helicases can either form, or not form, toroidal, hexameric rings (Singleton et al., 2007). Based on structural and functional analyses, all helicases are categorised into six major superfamilies (SFs) (Gorbalenya and Koonin, 1993). SF1 and SF2 include nonring forming helicases whereas the helicases that form ring-like structures are classified into SFs 3-6. SF1 and SF2 consist of characteristic helicase families that have high sequence conservation and all RNA helicases cluster within these families. A hallmark of RNA helicases belonging to SF1 and SF2 is the presence of a structurally conserved helicase core comprising two related domains arranged in tandem (Figure 1.4). These domains are similar to the fold of the bacterial recombination protein RecA (Singleton et al., 2007). Within the two RecA-like domains (D1 and D2), up to 12 highly conserved sequence motifs have been identified that have been found to play key roles in RNA substrate-binding, and NTPbinding and hydrolysis (Fairman-Williams et al., 2010). Motifs I (Walker A), II (Walker B) and $\mathrm{VI}$ are highly conserved and co-ordinate NTP-binding and hydrolysis. Motif I, classically defined as GxxxxGKT (Walker et al., 1982), requires the active participation of the three terminal residues (GKT). The side chain of the lysine interacts with $\beta$-phosphate of ATP and mutations in this motif lead to compromised NTP hydrolysis by the helicase (Pause and Sonenberg, 1992). Motif II consists of conserved amino acid stretches, aspartateglutamate-alanine-glutamate (DEAD) or aspartate-glutamate-alanine-histidine (DEAH). 
The glutamic acid residue is reported to act as a catalytic base during ATP hydrolysis whereas the carboxyl group of the aspartate is involved in the co-ordination of $\mathrm{Mg}^{2+}$ ion. Motif VI comprises two highly conserved arginine residues, involved in the stabilization of transition states during catalysis (Caruthers and McKay, 2002). The relatively recently identified $Q$ motif has been suggested to play a role in regulation of ATP-binding and hydrolysis and is absent in DEAH box helicases, which can potentially interact with other NTPs (Tanner et al., 2003). Additional conserved motifs include motifs III, which is involved in coupling NTP- and nucleic acid-binding, and motifs which primarily contact the RNA substrate (la, Ib, Ic, IV, IVa, V). These motifs have been also shown to play roles in bridging interactions between the RNA substrate and the NTP-binding site (Banroques et al., 2008).

A

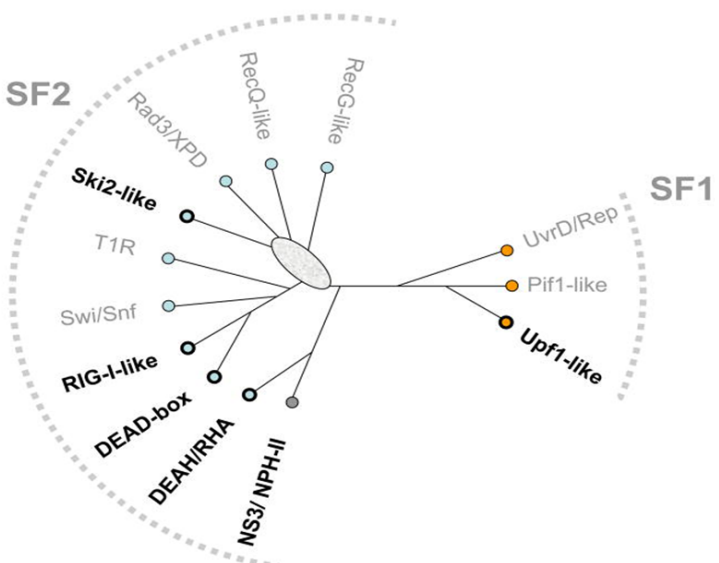

B

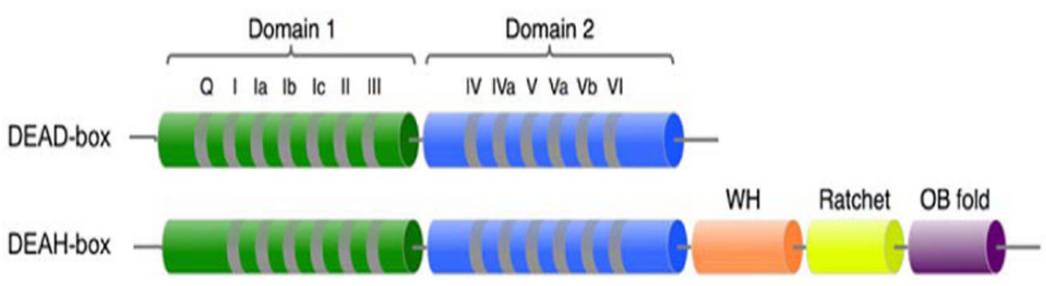

Figure 1.4. SF1 and SF2 superfamily of helicases. (A) RNA helicases cluster within 6 sub-families (Ski2, RIG-I-like, DEAD-box, DEAH/RHA, NS3/NPH-II, Upf1-like) of SF1 (right) and SF2 (left) superfamily of helicases (in bold) (Jankowsky,2011) (B) Domain architecture of DEAD-box and DEAH-box members of RNA helicases represents the conserved helicase core formed by two RecA-like domains (Domain1 and Domain 2). The helicase core constitutes of sequence motifs essential for NTP binding and hydrolysis. DEAD-box helicases don't possess conserved $\mathrm{N}$-or C-terminal extensions, however, C-terminal domain of DEAH-box helicases share conserved domains such as winged-helix $(\mathrm{WH})$, ratchet domain or the OBfold (panel adapted from Gilman et al., 2017).

Along with the conserved helicase core, RNA helicases frequently contain N- and Cterminal domains that are often larger than the helicase core in size and are highly variable within and across the helicase families (Fairman-Williams et al., 2010). As the core is highly 
conserved across all helicases, these terminal domains are suggested to play important roles in conferring substrate specificity. Additionally, they can assist in increasing the substrate repertoire of multi-functional helicases and can also serve as platforms for cofactor interactions (Karginov et al., 2005; Lattmann et al., 2010). The auxiliary domains can adopt distinct folds to confer additional functions such as nuclease activity, or serve as RNA-binding domains or protein-interaction domains (e.g. CARD domains) (Yoneyama and Fujita, 2008). The majority of the DEAD-box proteins have variable $\mathrm{C}$ - and $\mathrm{N}$-terminal domains typically including RNA recognition motifs (RRM) or zinc fingers (Linder, 2006). Interestingly, DEAD-box proteins can also exist with the minimal helicase core, lacking the terminal accessory domains (Rudolph and Klostermeier, 2015). In contrast, DEAH-box proteins all contain a highly conserved C-termini, which is organized into specific domain known as the OB-fold (oligonucleotide-binding), winged-helix domain and the ratchet domain. The winged-helix domain and the ratchet domain associate, and form stacking interactions with the nucleic acid base moieties (Walbott et al., 2010). The OB-fold includes five $\beta$-strands that form a $\beta$-barrel structure (Murzin, 1993) and can interact with nucleic acids or trans-acting protein cofactors (Arcus, 2002; Theobald et al., 2003)

\subsubsection{Mechanism of action of RNA helicases}

Although the three-dimensional structure of the core domains is similar between the DEADand DEAH-box family proteins, detailed structural studies have revealed that the mechanism of substrate binding and unwinding vary considerably between the two families (Gilman et al., 2017; Hamann et al., 2019; He et al., 2010; Tauchert et al., 2017).

DEAD-box proteins constitute the largest sub-group of the SF2 family with 37 members in humans and 26 in yeast (Fairman-Williams et al., 2010; Linder and Jankowsky, 2011). They are characterized by two flexible RecA-like domains interrupted by a linker and consist of a variable $\mathrm{N}$ - and $\mathrm{C}$-terminal tail region. Pioneering studies with the DEAD-box helicase elF4A, have demonstrated that DEAD-box helicases can interact directly with dsRNA and successfully unwind helices up to $15 \mathrm{bp}$, however, the efficiency of unwinding decreases with substrate length (Rogers et al., 1999). Hence, they are thought to act as local 'unwindase' with low processivity. Structural characterization of yeast DEAD-box Mss116, which is involved in RNA splicing of group I and II introns, has further revealed the detailed mechanism of action of this family of helicases (Figure 1.5A). 
A

DEAD-box

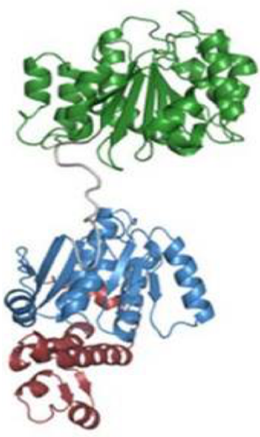

open 'inactive'

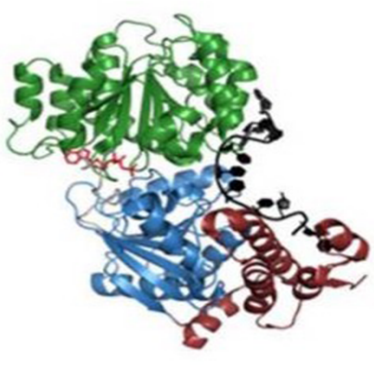

close 'active'
B

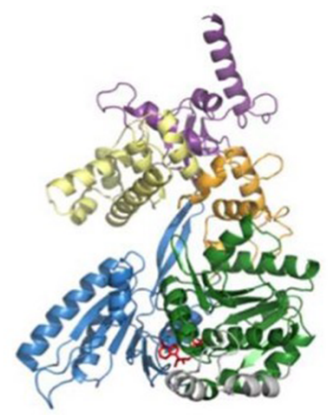

open 'inactive'
DEAH-box

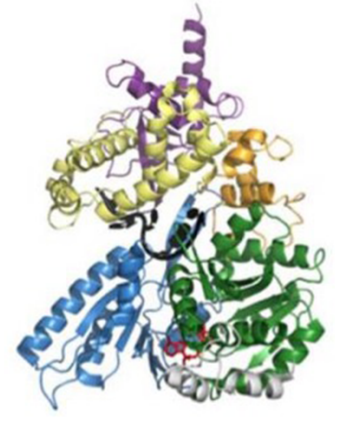

close 'active'

Figure 1.5. Structural characterization of DEAD-box and DEAH-box helicases. (A) Structure of DEAD-box helicase Mss116 in the 'open' conformation (left) based on small-X-ray scattering data and the 'close' conformation (right) derived from crystal structure (PDB: 315X). The structure of the 'closed' conformation was obtained in the presence of bound nucleotide (in red) and the ssRNA substrate (in black). Additional C-terminal domains present in Mss116 but not conserved amongst all DEAD-box proteins is represented in magenta (B) Structure of DEAH-box helicase Prp43 in the 'open' (left) and 'close' (right) conformation obtained from crystal structures (PDB: 5LTK and PDB: 5LTA, respectively). The bound nucleotide and sSRNA shown in red and black, respectively. All domains are coloured as in figure 3 (Panel adapted from Gilman et al., 2017).

In the absence of ligands, the two RecA-like domains of DEAD-box helicases are spatially separated and adopt an 'open' inactive conformation (Mallam et al., 2011, 2012).

Co-operative binding of ATP to D1 and the dsRNA substrate to D2 promotes structural rearrangement, resulting in the formation of a 'closed' active state, characterized by the intimate inter-domain contacts between the conserved sequence motifs. The closed conformation distorts the bound dsRNA by introducing a kink in one strand leading to duplex unwinding and release of the other stand by the complex. ATP hydrolysis and release of inorganic phosphate $\left(\mathrm{P}_{\mathrm{i}}\right)$ then facilitates the dissociation of the enzyme complex and release of the bound RNA strand (Hilbert et al., 2009; Yang et al., 2007). DEAD-box helicases, due to their low processivity, can unwind only short stretches of RNA by stochastic dissociation of double stranded helices around the sequence tightly bound by the helicase. Notably, a single round of ATP hydrolysis is only required for the recycling of the helicase but not for strand unwinding (Chen et al., 2008; Liu et al., 2008). In order to unwind longer RNA structures, DEAD-box helicases (for example, Mss16 or DDX43) can sometimes employ the assistance of their auxiliary domains. The basic C-terminal domain of Mss16 or the N-terminal domain of human DEAD-box protein, DDX43, have been shown to anchor the helicase close to their target sites, allowing multiple rounds of unwinding (Mohr et al., 2008; Talwar et al., 2017). 
In contrast, DEAH-box helicases are highly processive, and are able to translocate along unwind long RNA duplexes (Jarmoskaite and Russell, 2014; Pyle, 2008). Similar to DEADbox proteins, they comprise conserved RecA-like domains, however, DEAH-box helicases contain different sequences within the conserved motifs (Figure 1.5B). In addition, the conserved C-terminal domains of DEAH-box proteins have been shown to physically associate with the core domains, resulting in the formation of a relatively rigid structure in comparison to DEAD-box helicases (Pyle, 2008). Furthermore, NTP binding can lead to extensive structural rearrangement in the $\mathrm{C}$-terminal extension. This results in the opening of an RNA-binding tunnel located between the C-terminal and D2, facilitating substrate loading (Tauchert et al., 2017). In contrast to DEAD-box proteins, DEAH-box helicases require 3' single-stranded regions to serve as 'launch pads' for the effective loading of the helicase (Tanaka and Schwer, 2005) and they can utilize all four NTPs for efficient translocation (Fairman-Williams et al., 2010). Translocation along a substrate RNA duplex requires a diverse range of dynamic interactions between conserved motifs within the helicase. Upon binding to NTPs, DEAH-box helicases can advance in the 3 ' $\rightarrow 5$ ' direction as the conserved domains bind the substrate RNA in a defined order. D1 preferentially associates with 3' end of the RNA substrate whereas D2 interacts towards the 5' end, trapping the bound RNA. (He et al., 2017; Pyle, 2008; Tauchert et al., 2017) A $\beta$-hairpin structure from D2 stimulates unwinding by positioning itself between the last unpaired base and the first base-pair of the substrate duplex (Büttner et al., 2007). Hence, directional translocation occurs as the RNA duplex, locked in a defined geometry, is unwound as the helicase moves in steps, encompassing a new base from the 5' end while releasing one base from the 3' side (He et al., 2017). In vivo, RNA duplexes are often found in combination with bound proteins, forming large ribonucleoprotein particles. In order to gain access to dsRNA substrates, a 'winching' mechanism has been suggested for DEAH-box proteins. This involves loading of the helicase on an accessible single-stranded RNA region but upon encountering a physical block during translocation, continued pulling on the RNA strand, which ultimately results in the disruption of downstream base-pairing (Gilman et al., 2017; Semlow and Staley, 2012)

\subsubsection{Cellular pathways involving RNA helicases}

Traditionally, RNA helicases were considered to be an essential family of proteins catalysing the task of strand unwinding. Detailed biochemical and structural studies have revealed unexpected modes by which these proteins are able to carry out a diverse range of functions, and have now emerged as key players co-ordinating various aspects of RNA metabolism (Jarmoskaite and Russell, 2014; Linder and Jankowsky, 2011; Putnam and Jankowsky, 2013). Although the helicase-core domain does not inherently endow substrate 
specificity, in combination with various cis- and trans-acting factors, they can be involved in specific RNA-related processes such as translation, pre-mRNA splicing, ribosome biogenesis, RNA decay and pre-mRNA nuclear export (Bourgeois et al., 2016). While some RNA helicases are only implicated in one cellular process, many other RNA helicases appear to participate in a diverse range of processes (Jarmoskaite and Russell, 2014). Although, detailed mechanistic studies have revealed the precise functions of an increasing number of helicases, lack of identified target RNAs, coupled with uncharacterized binding partners, prove to be challenging obstacles for elucidating the physiological roles of RNA helicases.

\subsubsection{Role of RNA helicases in pre-mRNA splicing}

Pre-mRNA splicing is an essential nuclear event in eukaryotes leading to the removal of introns from the nascent transcript resulting in mature, translatable mRNAs. The removal of introns and the subsequent ligation of adjacent or distant exons (in case of alternative splicing) requires the involvement of over 100 proteins that facilitate the formation of the spliceosome (Fica and Nagai, 2017). The nascent pre-mRNA transcript, during RNA polymerase II-mediated synthesis, associates with RNA-binding proteins, triggering the recognition of splice sites (Bourgeois et al., 2016; Fu and Ares, 2014; Witten and Ule, 2011). Various RNA helicases mediate pre-mRNA splicing by either interacting directly with the core spliceosome machinery or are involved in the process of dynamic regulation. Eight helicases (DDX39B, DDX46, DDX23, Brr2, DHX16, DHX15, DHX8, DHX38) have so far been shown to associate with the spliceosome and assist in the formation of mature mRNAs by facilitating protein displacement, structural rearrangement, reaction intermediate stabilization, and ensuring the fidelity and directionality of this complex process (Cordin and Beggs, 2013; Fica and Nagai, 2017).

Apart from these proteins that modulate spliceosome assembly and function, a number of additional RNA helicases play important roles in regulating the mechanism of pre-mRNA splicing. For example, the DEAD box helicases, DDX5 and DDX17, control splicing events of a large number of exons (Dardenne et al., 2014). Likewise, DDX48 (elF4AIII) is a major constituent of the exon junction complex $(E J C)$ and can regulate alternative splicing (Michelle et al., 2012; Wang et al., 2014). Another DEAD-box helicase, DDX41 was shown to be an important moderator of alternate splicing; and mutations in DDX41 resulted in impaired splicing events (Polprasert et al., 2015).

\subsubsection{Role of RNA helicases in translation}

Eukaryotic cap-dependent translation initiation requires the disruption of secondary structures present in the 5' UTRs of mRNAs that can impede efficient scanning by the pre- 
initiation complex (PIC) (Aitken and Lorsch, 2012). Several RNA helicases have been implicated in resolving and restructuring 5'UTR regions, thereby promoting PIC loading and subsequent scanning. The DEAD-box helicase elF4A, assisted by other translation factors including elF4G (Rogers et al., 2001), facilitates association of the PIC with the mRNA by unwinding secondary structures in its vicinity (Aitken and Lorsch, 2012; Svitkin et al., 2001). Moreover, in yeast, efficient scanning by the initiation complex in cap-dependent initiation is also aided by the RNA helicase Ded1, which has been shown to stimulate (by promoting loading and scanning) or inhibit translational initiation (Hilliker et al., 2011). Apart from resolving 5'UTR structures, the DEAH-box helicase DHX29 can associate with the 40S ribosomal subunit near the mRNA entry channel and promotes the entry of mRNA by unwinding highly ordered structures (Abaeva et al., 2011). Furthermore, RNA helicases have also been shown to function at late stages of translation. The DEAH-box helicase DHX9 can modulate the translational process by anchoring to distinct secondary structures within mRNA and thereby promoting 40S scanning and mRNA circularization (Halaby et al., 2015; Manojlovic and Stefanovic, 2012). In addition, DDX33 and DDX19B are also key players in later steps of translation; DDX33 facilitates assembly of the 805 ribosomal complex (Zhang et al., 2015), and DDX19B is suggested to be involved in the recruitment of the eukaryotic release factor eRF3 (Gross et al., 2007).

\subsubsection{Role of RNA helicases in other cellular pathways}

In addition to their roles in translation and splicing, RNA helicases are central players in a variety of other cellular pathways. For example, nuclear export of mRNPs necessitates the formation of EJCs, which serve as binding platforms for factors essential for efficient export of mRNA into the cytoplasm and the formation of EJCs is facilitated by the action of DDX48 (elF4AIII) (Wang et al., 2014). A subset of mRNAs require specialized export adaptor proteins such as Aly/REF (ALYREF), which are recruited by the DEAD-box helicase DDX39B (Luo et al., 2001). Following successful transition through the nuclear pore complex (NPCs), mRNPs can undergo extensive structural and conformational rearrangements mediated by DDX19B (Tieg and Krebber, 2013). Apart from facilitating mRNP export, RNA helicases have been shown to be important for processes such as cytoplasmic mRNA decay and microRNA (miRNA)-induced gene silencing. mRNA decay in the cytoplasm is promoted by DDX6, which stimulates the de-capping process and facilitates mRNA degradation by the 5' - 3' exonuclease, XRN1 (Fischer and Weis, 2002; Tieg and Krebber, 2013). Nonsense-mediated decay requires the action of the SF1 helicase, UPF1 (Lykke-Andersen and Jensen, 2015). Cytoplasmic mRNA storage has evolved to be an alternate fate for mRNAs not undergoing translation or decay and the DEAD-box helicases DDX6 and DDX3X can enhance the formation of RNA storage 
granules in response to cellular stress (Hooper and Hilliker, 2013). Furthermore, miRNA biogenesis entails the processing of a primary miRNA (pri-miRNA) to form a precursor miRNA (pre-miRNA), which undergoes further processing to form the mature miRNA, involved in gene silencing. Helicases such as DDX5 and DDX17 (Moy et al., 2014) are involved in the processing of pri-miRNAs, demonstrating that RNA helicases also play important roles in the biogenesis of miRNAs. Additionally, ribosome biogenesis employs a vast network of RNA helicases that are necessary for the formation of mature, translationcompetent ribosomes (discussed in detail in section 1.3)

\subsubsection{Regulation of RNA helicases}

Although RNA helicases inherently lack substrate specificity and demonstrate very low rates of catalysis, they are known to be multi-functional and co-ordinate various cellular events (Bourgeois et al., 2016; Jarmoskaite and Russell, 2014). These features necessitate the involvement of trans-acting proteins that can regulate the activity of RNA helicases and can endow tight spatial and temporal control in the complex cellular milieu. Helicase regulation can be also achieved in cis via the $\mathrm{N}$ - and $\mathrm{C}$-terminal domains of RNA helicases or by post-translational modifications, which can result in the activation or inhibition of the helicase (Ozgur et al., 2015). Under the influence of these regulators, RNA helicases are able to associate with specific target RNA substrates, exhibit an increased rate of catalysis, and can be efficiently anchored to their sites of action (Sloan and Bohnsack, 2018).

\subsubsection{Cofactor-dependent regulation of RNA helicases}

A growing number of helicases have been shown to associate with proteins termed 'cofactors', which play important roles in modulating their functions in vivo. These cofactors can either be a heterogeneous group of proteins (stand-alone cofactors) that can regulate the activity of RNA helicases or may share a common domain via which they interact with the helicase. For example, the MIF4G family of proteins and the G-patch proteins act as cofactors for DEAD-box and DEAH-box helicases, respectively (Ozgur et al., 2015; RobertPaganin et al., 2015; Sloan and Bohnsack, 2018). DEAD-box proteins such as elF4A-like helicases are known to interact with cofactors containing evolutionarily-conserved MIF4G domains (Ponting, 2000). Structural studies have revealed that MIF4G domain-containing proteins, such as elF4G, can interact with the helicase-core region of elF4AI and its paralogue elF4All, stabilizing the 'half-open' conformation promoting RNA-binding and increasing the rate of catalysis during cap-dependent translation (Andreou and Klostermeier, 2014; Hilbert et al., 2011; Schütz et al., 2008). Interestingly, in addition to such stimulatory roles, MI4FG domain proteins such as CWC22 can also inhibit the activity of helicases. CWC22 binding to elF4AIII results in the reorientation of the helicase-core 
domains creating a distance between the ATP- and the RNA-binding sites, leading to the stabilization of an inactive conformation of the helicase (Alexandrov et al., 2012; Steckelberg et al., 2012). Therefore, MIF4G domain-containing proteins are able to interact with their specific cognate helicase and can induce opposing outcomes despite possessing high sequence and structural conservations. As a result, this family of proteins plays an important role in intricately balancing the activity of multi-functional enzymes such as elF4AIII. On the other hand, the C-termini of DEAH-box proteins consist of conserved sequence folds such as the OB-fold, which can serve as binding platforms for proteins (Arcus, 2002; Theobald et al., 2003). A family of effector proteins known as 'G-patch proteins' have been shown to interact with the OB-folds of specific DEAH-box helicases and regulate their activity (Robert-Paganin et al., 2015). G-patch proteins possess glycine rich stretches of 45-50 amino acid residues and are defined by the consensus sequence$\mathrm{HHX}_{3} \mathrm{GAX}_{2} \mathrm{GXGHGX}_{4} \mathrm{G}$ ( $\mathrm{H}$ - hydrophobic, A - aromatic, X - non-conserved amino acid, G conserved glycine) (Aravind and Koonin, 1999). In yeast, five G-patch proteins (Pfa1, Gno1, Spp382, Cmg1 and Spp2) have been shown to positively regulate the ATPase and unwinding activity of their cognate helicases (Boon et al., 2006; Chen et al., 2014a; Heininger et al., 2016; Lebaron et al., 2009; Tanaka et al., 2007; Warkocki et al., 2015).

The mechanistic details of helicase activation via G-patch domain-containing proteins are not fully understood. However, the binding of G-patch domains to OB-folds is thought to induce conformational changes that can influence the catalytic activity of the helicase (Christian et al., 2014; Heininger et al., 2016; Tanaka et al., 2007). Although these proteins were found to be conserved in higher eukaryotes, detailed studies have demonstrated that there are additional G-patch proteins found in higher eukaryotes (Chen et al., 2014b; Wen et al., 2008; Zang et al., 2014). This suggests that mammalian gene expression requires additional layers of RNA helicase regulation owing to a higher degree of complexity. MIF4G domain and presumably G-patch containing cofactors influence the activity of the helicase by triggering conformational changes in their tertiary structure. In contrast, several protein cofactors can modulate the activity of helicases without inducing structural rearrangements. As RNA helicases are RNA-dependent ATPases, these proteins can either inhibit (negative regulators) or promote (positive regulators) RNA binding to the cognate helicases (Napetschnig et al., 2009).

\subsubsection{Cofactor-independent regulation of RNA helicases}

In addition to functioning as binding platforms for cofactors, the $\mathrm{N}$ - and C-terminal domains flanking the helicase core can also contribute to helicase autoregulation by intrinsically stabilizing the core domains in an active/inactive conformation. In humans, the $\mathrm{N}$-terminal region of the DEAD-box helicase DDX19 interacts with the two RecA-like domains, thus 
stabilizing the inactive conformation. The $\mathrm{N}$-terminal domain is displaced upon RNA binding, which promotes the active state of the helicase (Collins et al., 2009). Apart from intramolecular regulation via auxiliary domains, some RNA helicases can also distinguish and preferentially associate with distinct RNA sequences. For example, the RNA recognition motif present in the C-terminal tail of the bacterial DEAD-box protein DbpA, is responsible for its specific recruitment to the 23S rRNA (Diges and Uhlenbeck, 2001). Additionally, the basic C-terminal tail of the DEAD-box helicase, Mss116 is crucial for its recruitment to the target RNA substrate (Mallam et al., 2012). The regulation of RNA helicases can also occur by post-translational modifications, which can either directly impact the catalytic activity or might influence protein-protein interactions (Sloan and Bohnsack, 2018). For example, acetylation of DDX21 has been shown to inhibit its unwinding activity and deacetylation can reinstate the helicase in its active form (Song et al., 2017). Recent data reveal that long non-coding RNAs (IncRNA) can also function as modulators of RNA helicase activity thereby, providing an additional layer of regulation necessary to regulate the dynamic functions of RNA helicases. For example, in dendritic cells, BC1 IncRNA modulates the activity of elF4AI during translation. BC1 has been reported to specifically inhibit the duplex unwinding activity of elF4AI while stimulating its ATP hydrolysis (Lin et al., 2008). Additionally, the IncRNA BC200 has been reported to serve as a cofactor for DEAH-box helicase $\mathrm{DHX} 36$, and regulates the unwinding of quadruplex containing RNA substrates (Booy et al., 2016).

\subsection{The roles of RNA helicases in ribosome biogenesis}

The process of ribosome biogenesis involves a dynamic interplay of a vast majority of factors including RNA helicases that play a key role in the remodelling of ribosomal subunits. In bacteria, four out of the five reported DEAD-box helicases have been implicated in ribosome biogenesis (Martin et al., 2013). In eukaryotes, the assembly of ribosomal subunits engages a large number of RNA helicases. These play a crucial role in mediating pre-rRNA folding and processing, and regulating the association of snoRNAs with preribosomal subunits and thereby remodelling protein-RNA and RNA-RNA interactions. Although several RNA helicases have been implicated in the process of ribosome biogenesis, the physiological roles of only a few, especially in higher eukaryotes, have been characterized in detail. As the process of ribosome biogenesis is largely conserved in eukaryotes, RNA helicases that have been well-characterized in yeast, are assumed to play similar roles in humans. Although this is true for a subset of RNA helicases, genome-wide screens and detailed characterization of specific helicases have highlighted that they might have additional or different roles in higher eukaryotes. 


\subsubsection{RNA helicases regulate snoRNA association with pre-ribosomal particles}

snoRNAs associate with pre-ribosomal subunits by forming base-pairing interactions with the pre-rRNA. These interactions either direct pre-rRNA modification or facilitate structural rearrangements of pre-ribosomal particles by mediating long-range interactions (Watkins and Bohnsack, 2012). So far, 72 of the 75 snoRNAs identified in yeast belonging to the box $\mathrm{C} / \mathrm{D}$ or H/ACA families, guide nucleotide modification and are non-essential. However, three snoRNAs, namely, U3, U14 and snR30, are essential and mediate pre-rRNA folding and cleavage (Rodríguez-Galán et al., 2013; Watkins and Bohnsack, 2012). snoRNA-guided restructuring and modification usually occurs early in the biogenesis pathway. The snoRNApre-rRNA interaction preserves the pre-rRNA in a stable, accessible conformation, to be acted upon by various trans-acting factors. Following snoRNA association and function, dissociation and subsequent removal from pre-ribosomal complexes is imperative for the formation of mature ribosomal subunits. Furthermore, snoRNA-mediated modifications often occur in clusters within distinct regions of the rRNA sequence. This implies that snoRNAs must dissociate in order to grant access to subsequent snoRNAs. In yeast, several of the 21 RNA helicases that associate with pre-ribosomal subunits have been implicated in promoting either binding or dissociation of specific snoRNAs from preribosomal complexes (Martin et al., 2013). U3 snoRNA is a central part of the SSU processome and forms long-range interactions with 18S rRNA sequences and with 5'ETS, close to the $A^{\prime}$ (humans) or $A_{0}$ (yeast) sites. These interactions play an important role in regulating the timing of formation of the central pseudoknot, a key structural element found in mature $40 \mathrm{~S}$ ribosomal subunits, and also for co-ordinating cleavage events within the 5 ' ETS and ITS1 region (Dutca et al., 2011; Sharma and Tollervey, 1999). In yeast, Dhr1 promotes the unwinding and displacement of the U3 snoRNA from the pre-ribosomal particles, allowing the downstream pre-40S maturation events to occur (Sardana et al., 2015; Zhu et al., 2016). The DEAD-box helicase Has 1 has been shown to play a prominent role in the displacement of the box C/D snoRNA U14, which is implicated in directing $18 \mathrm{~S}$ pre-rRNA processing and modification (Brüning et al., 2018; Enright et al., 1996; Li et al., 1990). The box H/ACA snoRNA snR30 is important for cell viability and co-ordinates early pre-rRNA cleavage events at sites $A_{0}, A_{1}$ and $A_{2}$ (Beltrame and Tollervey, 1995; Hughes and Ares, 1991; Li et al., 1990). The DEAD-box helicase, Rok1 was found to be required for its efficient release, promoting subunit maturation (Bohnsack et al., 2008). One of the best characterized helicases in the context of yeast ribosome biogenesis is Prp43, a multifunctional DEAH-box protein involved in various steps of ribosome assembly. It is required for the dissociation of a subset of box C/D snoRNAs, which direct the methylation of nucleotides in helices 32 to 35 in 25S rRNA (snR39, snR39b, snR50, snR59, snR60 and snR72) from pre-ribosomal subunits (Bohnsack et al., 2009). In vivo crosslinking data 
indicates that Prp43 directly interacts with the snoRNAs guiding methylation and also crosslinks to the snoRNA base-pairing sites in the pre-rRNA. This overlap suggests a direct role of Prp43 in the displacement of these snoRNPs from the pre-rRNA sequence (Bohnsack et al., 2009). Moreover, Prp43 is required for the binding of snoRNAs snR64 and snR67 to the pre-ribosomal subunit and snR67 mediated methylation of the 25S rRNA sequence (Bohnsack et al., 2009; Leeds et al., 2006).

In humans, the mechanistic details of snoRNA distribution on pre-ribosomal subunits is not yet well-studied. The DEAD-box helicase DDX51 associates with early pre-ribosomal particles and is implicated in the release of the box C/D snoRNA U8. The timely binding of U8 to the nascent the 47S pre-rRNA is important for the proper folding of the pre-rRNA transcript and the subsequent displacement of U8 is required for the efficient processing of the 3' end of the 28S rRNA sequence (Peculis and Steitz, 1993; Srivastava et al., 2010). Although, the functions and regulation of certain snoRNAs, for example U3 snoRNA, seem to be conserved in higher eukaryotes (Choudhury et al., 2019; Sardana et al., 2015), recent studies have highlighted the presence of additional snoRNAs lacking yeast counterparts. For example, The DEAD-box helicase DDX21 plays a prominent role in facilitating the preribosomal association of the late-acting box C/D snoRNAs SNORD56 and SNORD68, which are required for guiding methylation at specific sites within the 18S rRNA sequence. Although, the molecular details leading to the binding of these snoRNAs is not known, it has been suggested that DDX21 might be involved in the structural rearrangement of prerRNA transcript or in the removal of certain ribosome associated factors from the binding site of these snoRNAs. Notably, DDX21 as well as the rRNA modifications installed by SNORD56 and SNORD68 are not present in yeast, suggesting that specific RNA helicases might have co-evolved with their RNA targets (Sloan et al., 2015). Genome-wide screens have revealed the requirement of additional RNA helicases such as DDX10, DDX56 for efficient assembly of ribosomal subunits in human cells, however, the precise roles of these proteins in the context of ribosome biogenesis is yet to be established (Tafforeau et al., 2013; Turner et al., 2012; Wild et al., 2010).

\subsubsection{RNA helicases mediate structural remodelling of pre-ribosomes}

Apart from modulating the association of snoRNAs with pre-ribosomal particles, RNA helicases likely participate in structural rearrangement of pre-rRNA, promoting efficient cleavage or remodelling events during subunit maturation. The remodelling events induced by helicases could facilitate the stable incorporation of RPs, the release or binding of transacting RBFs, or promote the folding of pre-rRNA into mature secondary structures. For instance, in addition to its role in snoRNA release, Prp43 is implicated in the maturation of $18 \mathrm{~S}$ rRNA in yeast. During the final maturation steps in the cytoplasm, Prp43 likely induces 
structural remodelling of pre-ribosomal particles resulting in cleavage of the 3'end of $18 \mathrm{~S}$ pre-rRNA by the endonuclease Nob1 (Bohnsack et al., 2009; Pertschy et al., 2009). Although, the process of $18 \mathrm{~S}$ cleavage by Nob1 in the cytoplasm is conserved in higher eukaryotes (Preti et al., 2013; Sloan et al., 2013a), DHX15 (human homologue of Prp43) was not found to be required for the efficient processing of 18S rRNA (Sloan et al., 2019) but is instead required for efficient cleavage at the A' site within the 5' ETS (Memet et al., 2017). In yeast, deletion of the DEAD-box protein Dbp3 leads to pre-rRNA processing defects mirroring the defects observed upon knockdown of RNase MRP, which mediates cleavage at site $A_{3}$ in the ITS1 region (Weaver et al., 1997). It was therefore suggested that $\mathrm{Dbp} 3$ is involved in promoting structural rearrangement in the vicinity of site $A_{3}$, facilitating the recruitment of the RNase MRP (Kressler et al., 1999). Furthermore, Dbp6, together with $\mathrm{Dbp} 7$ and $\mathrm{Dbp} 9$, can promote the incorporation of the yeast large subunit ribosomal protein Rpl3 by extensive remodelling of the pre-60S ribosomal sub-complex (Daugeron and Linder, 2001; Rosado et al., 2007). In recent findings, the DEAD box helicase Spb4 has been implicated in the recruitment of pre-60S export adaptor protein, Arx1 (Brüning et al., 2018) and biogenesis factor Nog1 (Talkish et al., 2012), likely through structural rearrangement of the 25S pre-rRNA. As Nog1 functions as a placeholder for Nmd3, which is another pre-60S export adaptor protein (Matsuo et al., 2014; Sloan et al., 2016), Spb4 has been suggested to play a role in the efficient export of pre-60S subunits (Brüning et al., 2018). Mak5 binding to pre-60S particles has also been shown to be required for the incorporation of Rpl10 in the cytoplasm (Brüning et al., 2018), highlighting the importance of structural remodelling events carried out by RNA helicases in the maturation of ribosomal subunits. 


\section{Chapter Two: Scope of the thesis}

Eukaryotic ribosome biogenesis is one of the most energy-consuming and complex processes in the cell, involving more than 200 trans-acting factors including AAA-ATPases, GTPases, nucleases, snoRNPs and RNA helicases. These ribosome biogenesis factors play key roles in promoting pre-rRNA cleavage, folding and modification, as well as the structural remodelling of RNA-RNA and RNA-protein interactions within pre-ribosomal complexes. The pathway of ribosome biogenesis is largely conserved amongst eukaryotes and hence, most ribosome biogenesis factors characterised in yeast are expected to fulfil similar roles in higher eukaryotes. Although, this notion has been experimentally verified for a subset of assembly factors, RNAi-based genome-wide screens for human ribosome biogenesis factors and detailed characterization of some of the identified proteins imply that some proteins may have additional or different roles in comparison to their yeast counterparts. Despite the growing list of RNA helicases implicated in the process of human ribosome biogenesis, detailed functional characterization of many of these proteins is still lacking.

Hence, this study aimed to gain insight into the roles of the DEAH-box helicase DHX37 and the DEAD-box helicase DDX55 in the context of ribosome biogenesis.

The primary objectives of the study were to:

- Demonstrate the catalytic activity of DHX37 and DDX55 in vitro and characterize mutants.

- Determine the sub-cellular localisation of the candidate helicases.

- Analyse the requirement for DHX37 and DDX55 for pre-rRNA processing.

- Identify the binding sites of DHX37 and DDX55 on pre-ribosomal complexes or target RNAs in vivo.

- Understand the functional roles of DHX37 and DDX55 in maturation of the SSU and LSU, respectively.

- Explore potential modes of regulation of DHX37 and DDX55, including identification of cofactors proteins that influence their catalytic activity and/or determining the contribution of protein sequences beyond the conserved helicase core in regulating recruitment to target RNAs. 


\section{Chapter Three: Manuscript I}

Priyanka Choudhury, Philipp Hackert, Indira Memet, Katherine E. Sloan \& Markus T. Bohnsack (2019). The human RNA helicase DHX37 is required for release of the U3 snoRNP from pre-ribosomal particles, RNA Biology, 16, 54-68

\section{Author contributions:}

Figure 1: Immunofluorescence analysis of DHX37 localisation (PC); RNAi-mediated depletion analysis of DHX37 followed by western blotting (PC); sucrose density gradient centrifugation upon DHX37 depletion (PC); pulse-chase labelling assays for pre-rRNA detection (IM).

Figure 2: Northern blot analysis for pre-rRNA detection (PC); RNAi-mediated depletion of DHX37 and XRN2 (PC).

Figure 3: Recombinant expression and purification of $\mathrm{DHX} 37$ and $\mathrm{DHX} 37_{\mathrm{T} 282 \mathrm{~A}}(\mathrm{PC})$; in vitro ATP hydrolysis assay (PC); generation of cell line expressing DHX37-Flag and

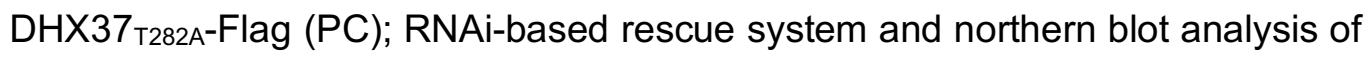
pre-rRNA levels (PC).

Figure 4: PAR-CRAC experiments for DHX37 (PH); native RNA-Immunoprecipitation and northern blotting for snoRNAs-U3 and U8 (PC).

Figure 5: Western blot analysis upon expressing DHX37-Flag and DHX37 T282A -Flag expression (PC); sucrose density gradient centrifugation and northern blotting for U3 snoRNA (PC).

Figure 6: Immunoprecipitation assays with Flag-tag and DHX37-Flag followed by western blotting for UTP14A, WBSCR22, tubulin (PC); recombinant expression and purification of UTP14A (PC); In vitro binding assay with DHX37 and UTP14A (PC); in vitro ATP hydrolysis assay (PC); RNAi-mediated depletion of UTP14A followed by western blotting (PC); northern blot analysis for pre-rRNA detection upon DHX37 and UTP14A depletion (PC); sucrose density gradient centrifugation and northern blotting for U3 snoRNA (PC). 
Figure S1: RNAi-mediated depletion of DIMT1L, TSR1, DHX37 followed by western blotting and northern blot analysis (PC).

Figure S2: Sucrose density gradient centrifugation and western blotting for DHX37 (PC).

Figure S3: Sequence alignment of DHX37 and Dhr1 (PC).

Figure S4: Immunoprecipitation assays with Flag-tag and DHX37-Flag followed by western blotting for DIMT1L, TSR1 and tubulin (PC).

Figure S5: in vitro ATP hydrolysis assay (PC).

Non-experiment contribution: Markus Bohnsack and Katherine Sloan conceived this project. Katherine Sloan, Markus Bohnsack and Priyanka Choudhury wrote the manuscript and organised the figures. Markus Bohnsack and Katherine Sloan supervised the study. All listed authors contributed to data analysis and preparation of the manuscript.

Digital Object Identifier: 10.1080/15476286.2018.1556149 


\section{The human RNA helicase DHX37 is required for release of the U3 snoRNP from pre-ribosomal particles}

Priyanka Choudhury ${ }^{1}$, Philipp Hackert ${ }^{1}$, Indira Memet $^{1}$, Katherine E. Sloan ${ }^{1 *}$ and Markus T. Bohnsack ${ }^{1,2^{*}}$

${ }^{1}$ Department of Molecular Biology, University Medical Centre Göttingen, Humboldtallee 23, 37073 Göttingen, Germany

${ }^{2}$ Göttingen Center for Molecular Biosciences, Georg-August University, Göttingen, Justusvon-Liebig-Weg 11, 37077 Germany

* Please address correspondence to:

Markus T. Bohnsack

Address: Department of Molecular Biology, University Medical Centre Göttingen, Humboldtallee 23, 37073 Göttingen, Germany

Phone: +49-551-395968; Fax: +49-551-395960

E-mail: markus.bohnsack@med.uni-goettingen.de

Katherine E. Sloan

Address: Department of Molecular Biology, University Medical Centre Göttingen, Humboldtallee 23, 37073 Göttingen, Germany

Phone: +49-551-395978; Fax: +49-551-395960

E-mail: katherine.sloan@med.uni-goettingen.de

Key words: RNA helicase, ribosome, ribosome biogenesis, snoRNA, protein cofactor, RNA processing 


\begin{abstract}
Ribosome synthesis is an essential cellular process, and perturbation of human ribosome production is linked to cancer and genetic diseases termed ribosomopathies. During their assembly, pre-ribosomal particles undergo numerous structural rearrangements, which establish the architecture present in mature complexes and serve as key checkpoints, ensuring the fidelity of ribosome biogenesis. RNA helicases are essential mediators of such remodelling events and here, we demonstrate that the DEAH-box RNA helicase DHX37 is required for maturation of the small ribosomal subunit in human cells. Our data reveal that the presence of DHX37 in early pre-ribosomal particles is monitored by a quality control pathway and that failure to recruit $\mathrm{DHX} 37$ leads to pre-rRNA degradation. Using an in vivo crosslinking approach, we show that DHX37 binds directly to the U3 small nucleolar RNA (snoRNA) and demonstrate that the catalytic activity of the helicase is required for dissociation of the U3 snoRNA from pre-ribosomal complexes. This is an important event during ribosome assembly as it enables formation of the central pseudoknot structure of the small ribosomal subunit. We identify UTP14A as a direct interaction partner of DHX37 and our data suggest that UTP14A can act as a cofactor that stimulates the activity of the helicase in the context of U3 snoRNA release.
\end{abstract}




\section{Introduction}

The production of eukaryotic ribosomes, which are responsible for the translation of mRNAs into proteins, is a highly complex, dynamic and energy-consuming cellular process involving the maturation of four ribosomal RNAs (rRNAs) and their assembly with approximately 80 ribosomal proteins (RPs). ${ }^{1}$ Numerous ribosome biogenesis factors (RBFs), which associate transiently with pre-ribosomal particles, are responsible for mediating key maturation events and in the yeast Saccharomyces cerevisiae, approximately 200 such factors have been identified (see for example reference 2). Due to the larger size of human (pre-) ribosomal complexes and the evolution of regulatory networks that coordinate ribosome production with other cellular processes, the inventory of human ribosome biogenesis factors is likely to be much larger than that of yeast. Proteomic analyses of human nucleoli, ${ }^{3,4}$ together with several recent genome-wide RNAi-based screens ${ }^{5-8}$ have uncovered a plethora of factors without yeast homologues that are required for human ribosome assembly. Furthermore, detailed analyses of the human pre-rRNA processing pathway (see for example references 9-11) and functional characterisation of various individual human RBFs have revealed that many human RBFs have different or additional functions compared to their yeast counterparts. A portfolio of genetic diseases, termed ribosomopathies, that are caused by mutations in genes encoding RPs and/or RBFs (reviewed in reference 12), as well as an increasing body of evidence linking ribosome assembly to cancer (reviewed in reference 13), emphasise the importance of a detailed knowledge of the human ribosome assembly pathway.

In human cells, ribosome assembly is initiated in the nucleolus by synthesis of a $47 \mathrm{~S}$ prerRNA transcript containing the sequences of three of the four rRNAs (18S, 5.8S and 28S), separated by internal transcribed spacers (ITS) and flanked by external transcribed spacers (ETS). Maturation of the pre-rRNAs involves processing of this transcript by endo- and exonucleases and extensive modification of the mature rRNA sequences by small nucleolar RNPs (snoRNPs) and stand-alone modification enzymes. ${ }^{14-16}$ A subset of RPs and RBFs are recruited to the nascent pre-rRNA transcript giving rise to an early pre-ribosomal complex termed the small subunit (SSU) processome. The SSU processome is composed of numerous sub-complexes, including the UTP-A, UTP-B and UTP-C complexes, as well as various enzymes that mediate important steps in SSU biogenesis, such as the endonuclease UTP24, the PIN domain protein UTP23, the rRNA methyltransferase EMG1 and the GTPase BMS1. ${ }^{6,17-19}$ A core component of the SSU processome is the U3 snoRNP, which in contrast to most other snoRNPs, does not guide rRNA modification, but instead bridges key interactions within early pre-ribosomes. ${ }^{20}$ This is achieved by base-pairing 
interactions between the U3 snoRNA 3' hinge region and the 5' ETS, and the U3 5' region ( $G A C$ box, box $A$ and box $A^{\prime}$ ) and the $18 \mathrm{~S}$ rRNA sequence that forms the central pseudoknot in mature SSU complexes. ${ }^{21-23}$ Recent structural analyses of $S$. cerevisiae SSU processomes ${ }^{24,25}$ have revealed that the base-pairing interactions formed between the U3 snoRNA and the pre-rRNA tether the SSU processome in a conformation that allows essential pre-rRNA processing events to be co-ordinated. Release of the U3 snoRNP, which in yeast requires the RNA helicase $\mathrm{Dhr} 1^{26}$, is an essential step in SSU biogenesis as it licences downstream maturation events and allows folding of the 18S rRNA sequence into its mature conformation. A pre-rRNA cleavage step in ITS1 separates the SSU (40S) and LSU (60S) precursor particles, which undergo separate maturation pathways in the nucleolus and nucleoplasm involving further pre-rRNA processing steps, the recruitment and release of various RBFs and significant structural rearrangements. Such remodelling events serve as important checkpoints during the assembly pathway and are often a prerequisite for establishing export competence. The pre-40S and pre-60S subunits are independently exported to the cytoplasm ${ }^{27}$ where final maturation and quality control steps take place before the mature subunits re-join during translation.

RNA helicases, which are typified by a helicase core domain composed of tandem RecAlike domains containing conserved sequence motifs involved in NTP/substrate binding, NTP hydrolysis and its coordination with unwinding activity, are proposed to be important regulators of pre-ribosome remodelling events. ${ }^{28,29}$ Structural rearrangements, such as the release of snoRNPs from pre-ribosome, recruitment or dissociation of RPs and/or RBFs, and altering pre-rRNA base-pairing/folding, are proposed functions of RNA helicases during the maturation of pre-ribosomal subunits. ${ }^{26,30-35}$ In human cells, the DEAD-box helicases DDX51 and DDX27 are implicated in release of the metazoan-specific snoRNA U8 from its base-pairing sites with the 28S rRNA and ITS2 sequences, and maturation of the 3' end of the $28 \mathrm{~S}$ rRNA respectively. ${ }^{36,37}$ Interestingly, the catalytic activity of the DEAH-box RNA helicase DHX15, which is the human homologue of yeast Prp43, is required for a metazoanspecific cleavage event (A') in the 5' ETS. ${ }^{38}$ However, unlike Prp43, DHX15 does not appear to be required for the final steps of $18 \mathrm{~S}$ rRNA maturation, implying that the functions of this helicase are different in yeast and humans. Furthermore, human ribosome assembly involves RNA helicases not present in yeast; for example, it was recently shown that the action of the metazoan-specific DEAD-box protein DDX21 is required for rDNA transcription and that this enzyme also facilitates the access of late-acting snoRNPs to pre-40S complexes. ${ }^{39,40}$ Recent cryo-EM structures of late pre-40S complexes ${ }^{41,42}$ provide the first snap-shots of structural rearrangements that take place during the final steps of SSU 
maturation but the roles of many human RNA helicases during ribosome assembly remain uncharacterised.

Here, we show that the putative RNA helicase DHX37 is an RNA-dependent ATPase that is required for biogenesis of the SSU. Our data show that lack of DHX37 triggers a surveillance pathway that leads to degradation of pre-ribosomal particles. Depletion of DHX37 inhibits the conversion of the 21S pre-rRNA to 18SE and expression of catalytically inactive DHX37 also impairs a pre-rRNA cleavage step in the 5' ETS. Using in vivo proteinRNA crosslinking, we demonstrate that DHX37 binds close to the 3 ' hinge region of the U3 snoRNA, which base-pairs with the 5' ETS in proximity of the A' cleavage site, and we show that expression of catalytically inactive DHX37 causes accumulation of the U3 snoRNA on pre-ribosomes. Together, our data imply that dissociation of the U3 snoRNA, which requires $\mathrm{DHX} 37$, is an important early pre-ribosome remodelling event that licences downstream steps in human ribosome assembly. 


\section{Results}

The human RNA helicase DHX37 is required for biogenesis of the small ribosomal subunit. Although many components of the ribosome assembly machinery are conserved throughout eukaryotes, the precise functions of many RBFs, especially RNA helicases, differ in yeast and humans. Based on its homology to yeast Dhr1, the putative RNA helicase DHX37 is anticipated to play a role in human ribosome biogenesis, however, a detailed functional characterisation of this protein is lacking. To first ascertain the localisation of DHX37 in human cells, we performed immunofluorescence in HeLa cells using antibodies against DHX37 and, as a nucleolar marker, the rRNA methyltransferase NSUN5. This revealed that DHX37 predominantly localises to nucleoli (Figure 1A), which is consistent with a role during the early stages of ribosome assembly. Next, to enable the effects of depletion of DHX37 on ribosome assembly to be analysed, RNAi against DHX37 was established using two alternative siRNAs. HeLa cells were left untreated (wild-type), or were transfected with control siRNAs or siRNAs targeting DHX37, then the levels of the DHX37 protein were examined using western blotting. This demonstrated that the amount of DHX37 in cells treated with either of the two siRNAs against DHX37 was markedly reduced (Figure 1B), thereby confirming the effectiveness of both siRNAs. To determine the effect of depletion of DHX37 on ribosomal subunit production, whole cell extracts prepared from cells treated with either non-target siRNAs (siNT) or those targeting DHX37 (siDHX37_1) were separated by sucrose density gradient centrifugation and the relative amounts of small subunits (40S), large subunits (60S) and monosomes (80S) were determined by monitoring the absorbance of each fraction at $260 \mathrm{~nm}$. The obtained profile shows that depletion of DHX37 does not affect production of 60S subunits but leads to a significant decrease in the abundance of $40 \mathrm{~S}$ particles as well as a decrease in the amount of 805 monosomes (Figure 1C), indicating a role for $\mathrm{DHX} 37$ in production of the small ribosomal subunit. Consistent with this, pulse-chase labelling of newly synthesised RNAs in siRNA-treated cells as described above confirmed the requirement for DHX37 for production of the 18S rRNA, but not the $28 \mathrm{~S}$ rRNA (Figure 1D and E). To determine at what stage of SSU production DHX37 is required, northern blotting was used to examine the levels of different pre-rRNA species in cells depleted of DHX37. Compared to RNA from wild-type cells or cells treated with nontarget siRNAs, depletion of DHX37 caused accumulation of the 21S pre-rRNA and a concomitant decrease in the levels of the 18SE pre-rRNA (Figures 1D and F), which is largely produced by exonucleolytic processing of the $21 \mathrm{~S}$ pre-rRNA following site 2 cleavage. ${ }^{10,11}$ Interestingly, several aberrant pre-rRNAs were also detected when DHX37 was depleted; in particular, prominent species that migrated below the 41S and 18SE prerRNAs were observed. 
The detection of these aberrant pre-rRNA species upon depletion of DHX37 raised the question of how they are produced. The pre-rRNA regions present in each of the prominent intermediates were therefore determined by northern blotting using a series of probes hybridising within the 5' ETS and ITS1 (Figure 2A). The 16S* species was detected with probes hybridising to ITS1 upstream, but not downstream, of site 2 (Figure 2B), implying that the $3^{\prime}$ end of this pre-rRNA is generated by site 2 cleavage. The finding that a probe hybridising within the 5' ETS, immediately upstream of site 1 was not able to detect $16 \mathrm{~S}^{*}$, together with the estimated size of this intermediate, indicates that its 5 ' end lies within the mature $18 \mathrm{~S}$ rRNA sequence. While the $38 \mathrm{~S}^{*}$ intermediate could be visualised using probes spanning ITS1, it was not detected with probes hybridising in the 5' ETS (Figure 2A and B), implying that, similarly, the 5 ' end of this species lies within the mature 18S rRNA sequence. A series of northern blot probes spanning the 18S rRNA sequence (Figure 2A) were

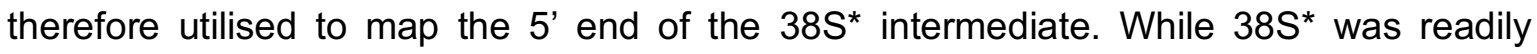
observed using probes 18S-1285, 18S-1377 and 18S-1670, it was only very weakly detected by probe $18 \mathrm{~S}-1230$ and was not visualised using probes $18 \mathrm{~S}-1$ or $18 \mathrm{~S}-1004$ (Figure $2 \mathrm{C}$ ). This suggests that the $5^{\prime}$ end of the $38 \mathrm{~S}^{*}$, and by analogy, the $16 \mathrm{~S}^{*}$ species, is somewhat heterogeneous but primarily lies between nucleotides 1253 and 1285 (helix 32 ) of the $18 \mathrm{~S}$ rRNA (Supplementary Figure S1A). The finding that the $38 S^{*}$ and $16 S^{*}$ intermediates are not part of the normal pre-rRNA processing pathway, but instead represent turnover intermediates derived from the $41 \mathrm{~S}$ and $30 \mathrm{~S} / 21 \mathrm{~S}$ pre-rRNAs respectively, suggests the existence of a surveillance pathway that targets pre-ribosomal complexes lacking DHX37 for degradation. Relatively little is known about how incorrectly assembled pre-ribosomes are turned over in human cells, however, an enzyme that has been implicated in the degradation of aberrant pre-rRNAs is the 5'-3' exonuclease XRN2. ${ }^{43}$ To determine if the $5^{\prime}$ ends of the $38 S^{*}$ and $16 S^{*}$ intermediates are generated by the stalling of XRN2 during exonucleolytic processing, pre-rRNA intermediates were examined in HeLa cells depleted of DHX37, XRN2 or both DHX37 and XRN2. As previously reported, depletion of XRN2 impaired A' cleavage, leading to accumulation of the 30SL5' intermediate ${ }^{10,43}$ and the previously observed defect in conversion of the $21 S$ pre-rRNA to 18SE was detectable when the levels of DHX37 were reduced both in the absence and presence of XRN2 (Figure 2C). Upon co-depletion of DHX37 and XRN2, the 38S* and 16S* fragments were clearly observed, implying that this surveillance pathway does not rely on XRN2 and that other endo-/exonuclease(s) mediate the degradation of pre-rRNAs within aberrant pre-ribosomes lacking DHX37.

Interestingly, the $38 \mathrm{~S}^{*}$ and $16 \mathrm{~S}^{*}$ pre-rRNA intermediates are also detected upon depletion of the rRNA methyltransferase DIMT1L, which installs two evolutionarily conserved $N^{6}-N^{6}$ - 
dimethylations at positions $\mathrm{A} 1850$ and $\mathrm{A} 1851$ of the $18 \mathrm{~S}$ rRNA sequence that forms the decoding centre in the mature SSU, and TSR1, which acts during the late stages of SSU biogenesis where it is proposed to prevent premature association of the small and large ribosomal subunits (Supplementary Figure S1B,C) ${ }^{6,44-46}$ This suggests that the presence of DHX37, DIMT1L and TSR1 in early pre-ribosomal complexes may all be monitored by a common surveillance pathway.

\section{DHX37 is an RNA-dependent ATPase and its catalytic activity is required for the conversion of the 21S pre-rRNA to the 18SE pre-rRNA.}

Several enzymes involved in ribosome biogenesis have been shown to have both catalytic and non-catalytic functions in the pathway (see for example references 47-49). Given the extensive pre-rRNA processing defects detected upon depletion of DHX37 and the fact that DHX37 is a putative DEAH-box RNA helicase, we next aimed to demonstrate that DHX37 is a catalytically active enzyme and determine if this activity is required for its function(s) in biogenesis of the small ribosomal subunit. A characteristic feature of RNA helicases is their capability to hydrolyse nucleotide triphosphates (NTPs) in an RNA-dependent manner 29,50 and we therefore expressed recombinant $\mathrm{His}_{6}$-tagged DHX37 in Escherichia coli and purified the protein for use in in vitro ATPase assays (Figure 3A). To confirm the specificity of any ATPase activity detected, DHX37 carrying a threonine to alanine substitution (T282A) in the evolutionarily conserved "GKT" sequence of motif $I$ that is implicated in NTP binding, ${ }^{26,50}$ was also expressed and purified (Figure 3A). NADH-coupled ATPase assays were performed using $\mathrm{DHX} 37$ or $\mathrm{DHX} 37_{\mathrm{T} 282 \mathrm{~A}}$ in the absence and presence of RNA. Compared to a control reaction containing neither protein nor RNA, DHX37 showed mild ATPase activity in the absence of RNA and the addition of RNA stimulated ATP hydrolysis by DHX37 almost four-fold (Figure 3B), demonstrating that DHX37 is an RNA-dependent ATPase. While DHX37 2 T282A. also weakly hydrolysed ATP in the absence of RNA, the effect of RNA addition on the activity of DHX37 $282 \mathrm{~A}$ was minimal, indicating that this amino acid substitution within the GKT motif impairs the catalytic activity of the helicase. To enable the requirement for the catalytic activity of $\mathrm{DHX} 37$ for small subunit biogenesis to be examined in human cells, HEK293 cell lines were generated in which endogenous DHX37 could be depleted using an established siRNA (Figure 1B) and a C-terminally His 6 -PreScission protease-2x Flag (Flag) tagged version of $\mathrm{DHX} 37$ or DHX37 T282A $_{2}$ could be expressed from a genomic locus via a tetracycline regulatable promoter. HEK293 cells were transfected with non-target siRNAs (siNT) or siRNAs targeting DHX37 (siDHX37_1) and expression of the Flag tag, DHX37-Flag or DHX37 T282A -Flag was induced (Figure 3C). Western blot analysis confirmed specific expression of the Flag-tagged proteins, albeit at slightly higher than endogenous levels. Examination of pre-rRNA levels in these cells by northern blotting 
using a probe hybridising to the 5' end of ITS1 demonstrated that depletion of DHX37 from HEK293 cells leads to pre-rRNA processing defects similar to those observed in HeLa cells (Figure 1F) and that expression of wild-type DHX37 from a transgene can rescue these defects (Figure 3D). Interestingly, the aberrant $38 S^{*}$ and $16 S^{*}$ pre-rRNA fragments did not accumulate upon expression of $\mathrm{DHX} 37_{\mathrm{T} 282 \mathrm{~A}}$ (Figure $3 \mathrm{D}$ ), implying the presence of the protein rather than its ATPase activity is sensed by the quality control pathway that monitors DHX37 recruitment to pre-ribosomal complexes. In contrast, the accumulation of the $21 \mathrm{~S}$ pre-rRNA and reduced level of the 18SE pre-rRNA observed upon depletion of DHX37 were also detected in cells expressing DHX37 $282 \mathrm{~A}$ (Figure 3D; left panel), demonstrating that lack of the catalytic activity of DHX37 is responsible for these defects. Expression of DHX37 also lead to accumulation of a pre-rRNA longer than $30 \mathrm{~S}$ that was not detected in cells lacking DHX37. Northern blotting with a probe hybridising close to the 5 ' end of the $47 \mathrm{~S}$ pre-rRNA transcript confirmed this species to be the 5' extended version of 30S, 30SL5' (Figure 3D; right panel), which accumulates when $A^{\prime}$ processing is impaired. These data suggest that while cleavage at the A' site in the 5' ETS can take place when DHX37 is lacking, the presence of inactive $\mathrm{DHX} 37$ in pre-ribosomal particles reduces the efficiency of this cleavage event or promotes bypassing of this step.

\section{DHX37 associates directly with the U3 snoRNA in vivo.}

Elucidating the precise functions of RNA helicases in ribosome biogenesis necessitates identification of the precise targets of their remodelling activity. To gain insight into the in vivo RNA substrates of DHX37, the crosslinking and analysis of $\underline{\mathrm{c} D N A}$ (CRAC) approach was applied. ${ }^{51,52}$ HEK293 cells expressing either the Flag tag or Flag-tagged DHX37 were grown in the presence of 4-thiouridine before in vivo crosslinking using light at $365 \mathrm{~nm}$. Cell extracts were used for tandem affinity purification under native and denaturing conditions, and a partial RNase digestion was performed to ensure that only RNA sequences directly contacted by DHX37 were recovered. RNA fragments co-purified with DHX37-Flag or the Flag tag were ligated to sequencing adaptors and labelled with $\left[{ }^{32} \mathrm{P}\right]$. RNA-protein complexes were separated by denaturing polyacrylamide gel electrophoresis (PAGE), transferred to a nitrocellulose membrane and then visualised by autoradiography (Figure 4A). A signal migrating at the molecular weight of DHX37 was detected in the sample derived from cells expressing DHX37-Flag but not the Flag tag, confirming the direct interaction of DHX37 with RNA in vivo. This area of the membrane, and a corresponding region for the control sample (Figure 4A), were excised and RNA fragments were isolated. After reverse transcription, the cDNA library was amplified by PCR and subjected to Illumina sequencing. The obtained sequence reads were mapped to the human genome using a filter to allow only mapping of reads containing a specific T-C mutation introduced during 
the reverse transcription step at positions where an amino acid remained crosslinked to a 4-thiouridine. Relatively few sequence reads mapped to the rDNA and no specific crosslinking site(s) could be identified. This suggests that, similar to yeast Dhr1 ${ }^{26}, \mathrm{DHX} 37$ interacts only transiently or unstably with pre-rRNA/pre-ribosomal complexes. Consistent with this, sucrose density gradient analysis of the distribution of DHX37 between preribosomal and non-ribosomal complexes showed that under normal conditions, only a small proportion of the helicase co-migrates with pre-ribosomal particles (Supplementary Figure 2). A higher proportion of sequence reads mapping to snoRNA genes was, however, observed in the DHX37-Flag CRAC dataset compared to the control, suggesting the association of DHX37 with snoRNA(s) (Figure 4B). Analysis of the relative distribution of sequence reads mapping to snoRNA genes in the two datasets highlighted an enrichment of reads corresponding to the U3 snoRNA (Figure 4C; Supplementary Table 1). To confirm the association of DHX37 with the U3 snoRNA, extracts from cells expressing Flag-DHX37 or the Flag tag were used in immunoprecipitation experiments and the amounts of the U3, and as a control the U8, snoRNAs present in the inputs and eluates were examined by northern blotting. While the U8 snoRNA was not retrieved in either eluate, the U3 snoRNA was specifically co-purified with Flag-DHX37 but not the Flag tag, further supporting the interaction of DHX37 with the U3 snoRNA in vivo (Figure 4D). We next examined the distribution of CRAC sequencing reads mapping to each nucleotide of the U3 snoRNA sequence. Almost no sequence reads were observed to be derived from the very 5 ' end of the snoRNA containing the U3-specific GAC and A/A' boxes, which basepairs with an $18 \mathrm{~S}$ rRNA sequence that forms part of the central pseudoknot in the mature SSU particle. Instead, the majority of sequence reads in the DHX37-Flag dataset were mapped towards the 3' end of the snoRNA containing the box C/D elements and close to the 3' hinge region of the U3 snoRNA that basepairs with pre-rRNA sequences present in the 5' ETS proximal to the A' cleavage site (Figure 4E; upper panel). The binding of DHX37 to these sequences is supported by the detection of T-C mutations in the sequencing reads as these are specifically introduced at nucleotides crosslinked to amino acids (Figure 4E; lower panel).

\section{The U3 snoRNA accumulates on pre-ribosomes when the catalytic activity of DHX37} is impaired.

The direct interaction of DHX37 with the U3 snoRNA suggests that, like its yeast counterpart Dhr1, DHX37 may regulate the interactions of this snoRNA with pre-ribosomal complexes. The amounts of $U 3$ present on pre-ribosomal complexes in cells expressing either wild-type DHX37 or catalytically inactive DHX37 $2282 \mathrm{~A}$ was examined. Endogenous DHX37 was depleted and expression of DHX37-Flag or DHX37 $2282-$ Flag was induced to equal levels (Figure 5A). Extracts from these cells were then subjected to sucrose density gradient 
centrifugation followed by northern blotting for the U3 snoRNA (Figure 5B). In cells expressing wild-type DHX37, the U3 snoRNA was present in fractions containing nonribosomal complexes as well as those containing larger pre-ribosomal complexes. Expression of $\mathrm{DHX} 37_{\mathrm{T} 282 \mathrm{~A}}$ lead to a notable increase in the proportion of the U3 snoRNA associated with pre-ribosomal complexes (Figure $5 \mathrm{~B}$ ), implying that the catalytic activity of $\mathrm{DHX} 37$ is required for release of this snoRNA from pre-ribosomal particles.

\section{DHX37 interacts directly with the SSU biogenesis factor UTP14A.}

The finding that the catalytic activity of DHX37 is required for release of the U3 snoRNA from pre-ribosomal particles raises the question of how this activity is regulated in the cellular environment. The promiscuity of many RNA helicases is limited by their inherently low catalytic activity, which is stimulated by dedicated cofactors upon binding to their cognate RNA substrates. Furthermore, protein cofactors can bestow specificity to RNA helicases by directing their recruitment to particular target RNAs in vivo (reviewed in 53). In yeast, Dhr1 physically interacts with the $18 \mathrm{~S}$ rRNA $\mathrm{m}^{7} \mathrm{G}$ methyltransferase Bud23 and the SSU biogenesis factor Utp14 to facilitate its access to pre-ribosomal particles, and it has been suggested that the unwinding activity of Dhr1 is stimulated by a direct interaction with Utp14. ${ }^{26,54,55}$ To determine if DHX37 interacts with the human orthologues of Bud23 (WBSCR22) and/or Utp14 (UTP14A) immunoprecipitation assays were performed. Extracts from cells expressing DHX37-Flag or the Flag tag were treated with RNase A or left untreated to determine if any interactions detected are bridged by RNA, and inputs (1\%) and eluates were analysed by western blotting. This revealed that neither tubulin nor WBSCR22 were recovered with DHX37-Flag in the presence or absence of RNase treatment (Figure 6A). In yeast, Bud23 interacts with amino acids in the $\mathrm{N}$-terminal region of Dhr $1^{55,56}$ and the lack of a direct interaction between DHX37 and WBSCR22 likely reflects the poor sequence conservation of this region of the helicase between yeast and humans (Supplementary Figure 3). Furthermore, in contrast to yeast Bud23, which is recruited to early pre-ribosomal complexes, WBSCR22 is reported to be recruited to late pre-40S complexes, ${ }^{57}$ consistent with the lack of an interaction with DHX37, which is likely to only be present in early complexes. UTP14A was, however, efficiently co-precipitated with DHX37-Flag, but not the Flag tag alone, implying the existence of pre-ribosomal complexes containing both these proteins (Figure 6A). The observed interaction between DHX37-Flag and UTP14A was not affected by RNase treatment, suggesting that this interaction is mediated by protein-protein contacts (Figure 6A). As depletion of DIMT1L or TSR1 appears to trigger the same quality control pathway as depletion of DHX37 (indicated by the accumulation of the aberrant $38 S^{*}$ and $16 S^{*}$ pre-rRNA species; Supplementary Figure 1), we speculated that these proteins might interact in human cells. Analysis of the IP inputs 
and eluates revealed that while both DIMT1L and TSR1 could be co-precipitated with DHX37-Flag from cell extracts, these interactions were dependent on RNA (Supplementary Figure 4A and B), implying that DHX37, DIMT1L and TSR1 are present in common preribosomal complexes but that they do not form direct interactions.

To test the hypothesis that UTP14A represents a direct interaction partner of $\mathrm{DHX} 37$, an in vitro binding assay was performed using recombinant ZZ-UTP14A-His ${ }_{10}$ and DHX37-His. DHX37-His 6 was incubated with either IgG sepharose or IgG sepharose on which ZZUTP14A-His 10 had been immobilised. After thorough washing steps, bound proteins were eluted and analysed by western blotting alongside input samples. DHX37-His 6 was coprecipitated with the ZZ-UTP14-His 10 -bound beads, but not with the empty lgG sepharose (Figure 6B), confirming that these proteins can interact directly. We next explored the influence of UTP14A on the catalytic activity of DHX37 using in vitro ATPase assays performed in the presence and absence of UTP14A. As previously shown (Figure 3B), DHX37 displayed RNA-dependent ATPase activity (Figure 6C) whereas the amount of ATP hydrolysed by UTP14A in the presence or absence of RNA was minimal. However, addition of UTP14A stimulated the RNA-dependent ATPase activity of DHX37 more than two-fold. To verify the specificity of UTP14A in stimulating the ATPase activity of DHX37, we also examined the influence of UTP14A on the ATPase activity of another DEAH-box RNA helicase, DHX15. While recombinant MBP-DHX15-His 10 displayed RNA-dependent ATPase activity, the rate of ATP hydrolysis was not stimulated by the addition of UTP14A (Supplementary Figure 5). To determine if UTP14A acts as a cofactor of DHX37 during SSU biogenesis, we first established RNAi against UTP14A (Figure 6D) and compared the pre-rRNA processing defects observed upon depletion of the helicase and its cofactor. Depletion of UTP14A caused accumulation of the 30S pre-rRNA and a corresponding decrease in the levels of the $21 \mathrm{~S}$ and $18 \mathrm{SE}$ pre-rRNAs (Figure $6 \mathrm{E}$ ). The aberrant $38 \mathrm{~S}^{*}$ and 16S* pre-rRNA fragments observed upon depletion of DHX37 did not accumulate when the level of UTP14A was reduced, suggesting that the presence of the helicase in pre-ribosomal particles, but not its cofactor, is monitored. The finding that depletion of UTP14A impedes a pre-rRNA processing step (conversion of $30 S$ to $21 S$ ) upstream of the processing event for which the catalytic activity of DHX37 is required (conversion of $21 \mathrm{~S}$ to $18 \mathrm{SE}$ ) could suggest that UTP14A has an additional role in SSU biogenesis that is not linked to its stimulation of DHX37. To investigate if, like DHX37, UTP14A is required for the release of the U3 snoRNA from pre-ribosomes, extracts from cells treated with control siRNAs (siNT) or siRNAs targeting UTP14A were separated by sucrose density gradient centrifugation and the amounts of U3 co-migrating with non-ribosomal and (pre-)ribosomal complexes were determined by northern blotting. Similar to expression of catalytically inactive DHX37 (Figure 5B), depletion of UTP14A lead to increased amounts of the U3 snoRNA present in 
fractions containing pre-ribosomal complexes (Figure 6F), suggesting that stimulation of DHX37 by UTP14A may be linked to the requirement for the helicase for the release of U3 from pre-ribosomes. 


\section{Discussion}

Here, we have analysed the putative RNA helicase DHX37, which is the homologue of the yeast DEAH-box protein Dhr1. In yeast, Dhr1 is required during the early steps of SSU maturation where it is proposed to trigger dissociation of the U3 snoRNA from its pre-rRNA base-pairing site(s) allowing formation of the central pseudoknot, a key structural feature of the $18 \mathrm{~S}$ rRNA within the small ribosomal subunit. ${ }^{26}$ Our data demonstrate that in human cells, DHX37 is also required for pre-40S maturation and that lack of DHX37 impairs various steps in the maturation of the 18S rRNA leading to reduced levels of both the mature $18 \mathrm{~S}$ rRNA and 40S subunits. We further show that DHX37 crosslinks to the U3 snoRNA and that expression of catalytically inactive DHX37 leads to increased levels of U3 on preribosomal complexes, suggesting that the function of this helicase in facilitating U3 snoRNA release may be conserved from yeast to humans. In yeast, changes in the solvent accessibility of a residue within the $18 \mathrm{~S}$ sequence that basepairs with the 5 ' region of the U3 snoRNA have been observed upon Dhr1 depletion, and Dhr1 has been reported to crosslink to the 5' region of the U3 sequence containing the GAC and A/A' boxes. ${ }^{26}$ However, in vitro Dhr1 has also been shown to unwind a duplex formed between the U3 3' hinge region and the corresponding 5 ' ETS sequence. ${ }^{26}$ It therefore remains unclear whether the remodelling activity of Dhr1 is directed at both of these U3-pre-rRNA duplexes or if disruption of one interaction is sufficient to trigger spontaneous dissociation of the U3 snoRNA from the other site leading to release of the snoRNA from early pre-ribosomal complexes. This open question is likely best addressed by structural analyses of Dhr1containing pre-ribosomal complexes; however, Dhr1 is not present in any of the currently available high resolution structures of SSU processomes, as these particles still contain the U3 snoRNA and evidence suggests that Dhr1 does not interact stably with preribosomes. ${ }^{24-26}$ Interestingly, in vivo crosslinking in human cells revealed direct interactions between DHX37 and U3 sequences that lie close to the 3' hinge region, which is predicted to basepair with the 5' ETS, implying that DHX37 may influence U3-5' ETS interactions. Consistent with this, we observed that expression of catalytically inactive DHX37 impairs processing at the $A^{\prime}$ cleavage site in the 5' ETS, a pre-rRNA cleavage event that has previously been shown to be sensitive to depletion of the U3-associated protein U3-55K (RRP9). ${ }^{58}$ Our complementation assays show that the catalytic activity of DHX37 is required for the conversion of the $21 \mathrm{~S}$ pre-rRNA to 18SE, indicating that removal of the 5 ' ETS and site 2 cleavage in ITS1 can take place but that subsequent processing of ITS1 is inhibited. The finding that removal of the 5' ETS and site 2 processing can take place although $A^{\prime}$ is impaired is consistent with previous data showing that $A^{\prime}$ cleavage can be bypassed. ${ }^{9}$ The pre-rRNA processing defects observed upon depletion of $\mathrm{DHX} 37$ could suggest that failure 
to release the U3 snoRNA during earlier stages of pre-40S maturation may not inhibit prerRNA processing until later. However, it is not yet known at precisely which stage of ribosome biogenesis the U3 snoRNA is released from human pre-ribosomes. Although production of the 21S pre-rRNA involves cleavages in 5' ETS, which would be anticipated to remove $\mathrm{U} 3$ base-pairing sites, it remains to be determined how $\mathrm{U} 3$ release is co-ordinated with pre-rRNA processing events. An alternative possibility is that the requirement for DHX37 for the conversion of 21S to 18SE represents an additional function for the helicase in SSU biogenesis, beyond its proposed role in facilitating U3 dissociation.

Similar to yeast Dhr1, DHX37 does not appear to associate stably with pre-ribosomal complexes in sucrose density gradient experiments. Nevertheless, the binding of DHX37 to the U3 snoRNA observed by immunoprecipitation and CRAC is anticipated to take place in the context of pre-ribosomal particles. Consistent with this, our complementation assays suggest that the presence of $\mathrm{DHX} 37$ in early pre-ribosomal complexes is monitored and that in the absence of the helicase, pre-ribosomes are targeted for degradation. Relatively little is known about the quality control pathways that are responsible for turnover of aberrant pre-ribosomes, especially in human cells, but it has been suggested that exonucleases involved in pre-rRNA processing steps could be utilised as degradation machines if particular ribosome biogenesis steps have not taken place correctly. Mapping of the aberrant $38 \mathrm{~S}^{*}$ and $16 \mathrm{~S}^{*}$ pre-rRNAs detected upon depletion of DHX37 demonstrates that they are 5' truncated derivatives of the $41 \mathrm{~S}$ and $21 \mathrm{~S}$ pre-rRNA, but depletion of the 5'3' exonuclease XRN2 did not influence production of these species. This implies that other nucleases are involved in the turnover of pre-ribosomes lacking DHX37. Stalling of exonucleases at highly structured pre-rRNA sequences, or pre-rRNA-bound ribosome biogenesis factors or ribosomal proteins may lead to formation of discrete pre-rRNA intermediates, and the diffuse nature of the $16 \mathrm{~S}^{*}$ intermediate, which often appears as two bands, suggests that this species may be heterogenous as would be expected if it is produced by exonucleolytic trimming. It is possible however, that during surveillance, the 18S rRNA sequence is first targeted by an endonuclease, as was recently reported in yeast. $^{58}$ In yeast, Utp24 cleaves the 18S rRNA sequence at nucleotide 618 (Q1 site) between helices 19 and 20 , whereas the $5^{\prime}$ end of $38 S^{*}$ maps to helix 32 of the human $18 \mathrm{~S}$ rRNA. While both these rRNA sequences lie in close proximity to the Utp24 binding site identified in yeast, as UTP24 is a core component of the SSU processome, expression of a catalytically inactive form of UTP24 would be required to investigate its potential role in preribosome quality control in human cells.

The finding that pre-ribosomes lacking $\mathrm{DHX} 37$ are targeted for degradation further highlights the importance of this protein for ribosome assembly. Interestingly, the surveillance pathway also appears to be triggered when the pre-40S biogenesis factors 
DIMT1L or TSR1 are lacking. While our data indicate that DHX37, DIMT1L and TSR1 can be present in the same pre-ribosomal particles, their association in vivo is dependent on RNA, implying that they do not form a discrete complex whose presence is monitored by this surveillance system. It is possible that the quality control pathway instead monitors maturation or folding of the central domain of the 18S rRNA as human TSR1 and yeast Dim1 have been demonstrated to bind this region, and DHX37 is implicated, either directly or indirectly, in regulating assembly of the central pseudoknot structure via U3 release. Alternatively, as the evolutionarily conserved rRNA modifications installed by DIMT1L are implicated in maintaining the fidelity of translation, and TSR1 plays an important role in preventing premature subunit joining, the requirement for a quality control pathway to confirm the pre-ribosomal recruitment of these three factors may reflect the critical roles they individually play during ribosome assembly.

In yeast, Dhr1 interacts with the rRNA methyltransferase Bud23 and with the pre-40S biogenesis factor Utp14. ${ }^{54-56}$ While Dhr1 has been shown to be required for the recruitment of Bud23 to pre-ribosomes, Bud23 and Utp14 are also necessary for the association of Dhr1 ${ }^{55,56}$ As these proteins also form direct interactions, it has been proposed that they are recruited to pre-ribosomes as a complex. Our data reveal that although the interaction between Dhr1/DHX37 and Utp14/UTP14A is conserved between yeast and humans, DHX37 does not co-precipitate the Bud23 orthologue WBSCR22 from human cells. On a molecular level this difference can readily be explained as structural analysis of the Dhr1Bud23-Trm112 complex revealed that amino acids in the N-terminal region of Dhr1 contact Bud $23^{55}$ and this region of the protein is largely absent from human DHX37. In yeast, Bud23 is recruited to early pre-ribosomal complexes but is reported to install the $18 \mathrm{~S}-\mathrm{m}^{7} \mathrm{G} 1575$ modification at a later stage ${ }^{55,59}$ In contrast, and consistent with the lack of interaction with DHX37, WBSCR22 is only present in later pre-40S complexes, ${ }^{38,57}$ implying that preribosomal recruitment of these two proteins is independent in human cells. However, as in yeast, we discovered a direct, RNA-independent interaction between DHX37 and UTP14A in human cells. The interaction surface between yeast Dhr1 and Utp14 has been identified and amino acid substitutions within this region have been shown to impair the association of these proteins. ${ }^{56}$ Surprisingly, introduction of the equivalent amino acid substitutions in DHX37 did not block its interaction with UTP14A (data not shown), suggesting that the precise contacts between these proteins are different in humans than yeast. Yeast Utp14 has been reported to stimulate the unwinding, but not ATPase, activity of the Dhr1 in vitro and is proposed to act as a cofactor for the helicase. ${ }^{26}$ The precise mechanism by which Utp14 regulates Dhr1 therefore remains unclear. We observe a mild increase in the rate of ATP hydrolysis by DHX37 in the presence of UTP14A compared to the helicase alone, suggesting that human UTP14A can also function as a helicase cofactor. Depletion of either 
yeast Utp14 ${ }^{56}$ or human UTP14A causes accumulation of the U3 snoRNA on preribosomes. As lack of UTP14A causes defects in early pre-rRNA processing steps, we cannot rule out the possibility that the observed accumulation of U3 on pre-ribosomes is an indirect effect cause by impaired ribosome assembly. However, taken together with the stimulation of DHX37 by UTP14A and data from a yeast complementation system showing that disruption of the interaction between these proteins causes a similar phenotype, it is likely that, on a cellular level, UTP14A acts together with DHX37 to promote U3 release.

Taken together, while our analysis of $\mathrm{DHX} 37$ suggests that the requirement for this helicase for efficient dissociation of the U3 snoRNP from pre-ribosomes is conserved in eukaryotes, we also highlight differences in the precise RNA and protein interactions formed by this helicase in yeast and human cells. To gain further mechanistic insights into Dhr1/DHX37 regulation by Utp14/UTP14A and to understand precisely how Dhr1/DHX37 contributes to U3 release, structural analyses of the helicase alone and within the context of preribosomes will likely be required. 


\section{Materials and methods}

\section{Molecular cloning}

The coding sequence of DHX37 (NM_032656.3) was cloned into a pcDNA5-based vector for the expression of proteins with a C-terminal $\mathrm{His}_{6}$-PreScission protease cleavage site2xFlag (Flag) tag from a defined genomic locus via a tetracycline-regulatable promoter in human cells. To express RNAi-resistant DHX37, site-directed mutagenesis was used to introduce 5 silent mutations within the binding site of siDHX37_1 (Supplementary Table 2). The coding sequences of DHX37 and UTP14A (NM_006649.3) were also cloned into pQE80-derivatived vectors (A42 and A15) for expression of proteins with a C-terminal His 6 tag or an N-terminal ZZ tag and C-terminal His 10 tag from IPTG-regulatable promoters in $E$. coli. Site-directed mutagenesis was performed on both the DHX37 constructs to introduce point mutations that lead to a threonine 282 to alanine substitution (T282A) in the expressed protein.

\section{Human cell culture, generation of cell lines and RNAi}

Hela CCL2 and HEK293 cells were cultured according to standard protocols at $37{ }^{\circ} \mathrm{C}$ with $5 \% \mathrm{CO}_{2}$ in $1 \times$ Dulbecco's Modified Eagle Medium (DMEM) supplemented with $10 \%$ foetal calf serum. To generate cell lines for expression of RNAi-resistant, wild-type or mutant DHX37, or the Flag tag, HEK293 Flp-In-T-Rex (Thermo Scientific) cells were transfected with the appropriate pcDNA5 constructs according to the manufacturer's instructions. Selection of stably transfected cells was achieved using hygromycin and blasticidin. Protein expression was induced by addition of $0.5-1000 \mathrm{ng} / \mathrm{mL}$ tetracycline for $24 \mathrm{~h}$ before harvesting. For RNAi, cells were transfected with 20-50 nmol of siRNA (Supplementary Table 2) using lipofectamine RNAiMAX (Thermo Scientific) according to the manufacturer's instructions. Cells were harvested $72 \mathrm{~h}$ after transfection unless otherwise stated.

\section{Immunofluorescence}

Immunofluorescence was essentially performed as previously described in ${ }^{19}$. HeLa cells were grown on coverslips before fixing using $4 \%$ paraformaldehyde in PBS for 10 min at room temperature. Cells were first permeabilised using $0.1 \%$ Triton-X-100 in PBS for 20 min then blocked with $10 \%$ FCS and $0.1 \%$ Triton-X-100 in PBS for $1 \mathrm{~h}$ at room temperature. Cells were incubated with anti-DHX37 and anti-NSUN5 antibodies (Supplementary Table 3) diluted in $10 \%$ FCS in PBS for $2 \mathrm{~h}$ at room temperature. Coverslips were washed and cells were incubated with Alexa Fluor 488-conjugated and Alexa Fluor 657-conjugated secondary antibodies for $1 \mathrm{~h}$ at room temperature. Coverslips were washed again and then 
mounted using Vectashield (Vector Laboratories) containing DAPI. Cells were analysed by confocal microscopy using a ConfoCor2 microscope (Carl Zeiss).

\section{RNA extraction, northern blotting and pulse-chase metabolic labelling}

Total RNA was extracted from human cells using TRI reagent (Sigma Aldrich) according to the manufacturer's instructions. Detection of pre-rRNAs by northern blotting was performed as previously described in ${ }^{9}$. In brief, $5 \mu \mathrm{g}$ of total RNA were separated on a $1.2 \%$ agaroseglyoxal gel and transferred to a nylon membrane by vacuum blotting or alternatively, RNAs were separated on a $10 \%$ denaturing ( $7 \mathrm{M}$ urea) polyacrylamide gel and transferred to nylon membrane by wet electroblotting. Membranes were pre-hybridised in SES1 (0.25 M sodium phosphate $\mathrm{pH} 7.0,7 \%$ SDS (w/v), 1 mM EDTA) buffer before incubation with 5' [ ${ }^{32} \mathrm{P}$ labelled DNA oligonucleotides (Supplementary Table 4) in SES1 buffer at $37^{\circ} \mathrm{C}$ overnight. After washing, signals were visualised using a Typhoon FLA9500 phosphorimager. Pulsechase labelling of nascent RNAs was essentially performed as previously described in ${ }^{10}$. siRNA-treated cells were grown in phosphate-free DMEM for $1 \mathrm{~h}$ before exchanging the media for phosphate-free DMEM supplemented with $15 \mu \mathrm{Ci} / \mathrm{mL} \quad\left[{ }^{32} \mathrm{P}\right]$-labelled orthophosphate and growth for a further $1 \mathrm{~h}$. The labelled media was then removed, cells were washed and grown in unlabelled DMEM for an additional $3 \mathrm{~h}$ before harvesting. Total RNA was extracted, separated by denaturing agarose gel electrophoresis, transferred to a nylon membrane by capillary blotting and abundant, labelled RNA species were visualised using a phosphorimager.

\section{Immunoprecipitation of complexes from human cells}

Expression of DHX37-Flag or the Flag tag was induced in HEK293 cells for $24 \mathrm{~h}$ and cells were harvested. Cells were lysed by sonication in a buffer containing $50 \mathrm{mM}$ Tris- $\mathrm{HCl} \mathrm{pH}$ 7.8, $150 \mathrm{mM} \mathrm{NaCl}, 0.5 \mathrm{mM}$ ethylenediaminetetraacetic acid (EDTA), $5 \mathrm{mM} \beta-$ mercaptoethanol and extracts were centrifuged at $20,000 \times \mathrm{g}$ for $10 \mathrm{~min}$ to remove insoluble material. The cleared lysate was supplemented with $1.5 \mathrm{mM} \mathrm{MgCl}_{2}$ and was either treated with RNase $\mathrm{A}(2.5 \mathrm{U})$ or was left untreated. The extracts were then incubated with anti-Flag magnetic beads (Sigma Aldrich) for $2 \mathrm{~h}$ at $4{ }^{\circ} \mathrm{C}$, and bound proteins and RNAs were eluted with $250 \mu \mathrm{g} / \mathrm{mL}$ Flag peptide. Proteins were precipitated with $20 \%$ trichloroacetic acid (TCA) and analysed by western blotting using antibodies listed in Supplementary Table 3. Alternatively, RNAs were extracted using phenol:chloroform:isoamylalcohol ( $\mathrm{PCl} ; 25: 24: 1)$, ethanol precipitated and analysed by northern blotting using probes listed in Supplementary Table 4. 


\section{Sucrose density gradient centrifugation}

HeLa cells, or HEK293 cell lines expressing DHX37-Flag or DHX37T282-Flag were lysed by sonication in a buffer containing $50 \mathrm{mM}$ Tris $\mathrm{pH} 7.5,100 \mathrm{mM} \mathrm{NaCl}, 5 \mathrm{mM} \mathrm{MgCl} 2$ and $1 \mathrm{mM}$ DTT before centrifugation at $20,000 \times \mathrm{g}$ for $10 \mathrm{~min}$ at $4^{\circ} \mathrm{C}$ to remove insoluble material. The cleared extracts were separated on 10-45\% sucrose density gradients in an SW-40Ti rotor for $16 \mathrm{~h}$ at 23,500 rpm. $530 \mu \mathrm{L}$ fractions were collected and the absorbance of each fraction at $260 \mathrm{~nm}$ was determined using a nanodrop (Peqlab). The proteins present in each fraction were precipitated using 20\% TCA, separated by SDS-PAGE and analysed by western blotting using antibodies listed in Supplementary Table 3. RNAs present in each fraction were extracted using $\mathrm{PCl}$, ethanol precipitated and analysed by northern blotting using probes listed in Supplementary Table 4.

\section{Crosslinking and analysis of cDNA (CRAC)}

CRAC was performed as previously described in references $38,40,51,52$. Briefly, expression of DHX37-Flag or the Flag tag was induced in HEK293 cell lines for $24 \mathrm{~h}$ before growth in media supplemented with $100 \mu \mathrm{M}$ 4-thiouridine for $6 \mathrm{~h}$. In vivo crosslinking was then performed using 2 cycles of $180 \mathrm{~mJ} / \mathrm{cm}^{2}$ light at $365 \mathrm{~nm}$ in a Stratalinker (Agilent Technologies). Cells were harvested and RNA-protein complexes were enriched by tandem affinity purification on anti-Flag M2 magnetic beads and NiNTA. After a limited RNase digestion, co-precipitated RNAs were 5 ' end labelled with $\left[{ }^{32} \mathrm{P}\right]$ and sequencing adaptors were ligated. RNA-protein complexes were separated by denaturing PAGE, transferred to a nitrocellulose membrane and labelled RNAs were visualised by autoradiography. Regions of the membrane were excised and RNAs were eluted by Proteinase $\mathrm{K}$ digestion then extracted using PCI. RNA fragments were reverse transcribed, amplified by PCR and the resulting library was subjected to Illumina sequencing. The obtained sequence reads were mapped to the human genome (GRCh37.p13).

\section{Purification of recombinant proteins, and in vitro binding and ATPase assays}

Expression of DHX37-His ${ }_{6}$ or ZZ-UTP14A-His 10 was induced in BL21 codon plus cells by addition of $1 \mathrm{mM} \mathrm{IPTG}$ for $16 \mathrm{~h}$ at $18^{\circ} \mathrm{C}$. Cells were pelleted, resuspended in Lysis buffer (50 mM Tris- $\mathrm{HCl} \mathrm{pH} \mathrm{7.0,500} \mathrm{mM} \mathrm{NaCl,} 1 \mathrm{mM} \mathrm{MgCl}, 10 \%$ glycerol and $5 \mathrm{mM}$ imidazole) and disrupted by sonication. The cell lysate was cleared by centrifugation at 50,000 $\mathrm{x} g$ for $30 \mathrm{~min}$ and the soluble fraction was incubated with cOmplete His-tag purification resin (Roche) for $2 \mathrm{~h}$ at $4^{\circ} \mathrm{C}$. After thorough washing steps with low and high salt buffers (50 mM Tris- $\mathrm{HCl} \mathrm{pH} \mathrm{7.0,500} \mathrm{mM/1000} \mathrm{mM} \mathrm{NaCl,} 1 \mathrm{mM} \mathrm{MgCl}, 10 \%$ glycerol and $25 \mathrm{mM}$ imidazole), bound proteins were eluted with lysis buffer supplemented with $500 \mathrm{mM}$ imidazole. The 


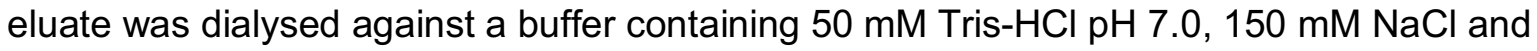
$20 \%$ glycerol. MBP-DHX15-His 10 was prepared as previously described in reference 38.

For in vitro binding assays, 200 pmol of recombinant ZZ-UTP14A-His 10 was immobilised on IgG sepharose (GE Healthcare) that had been pre-equilibrated in $50 \mathrm{mM}$ Tris- $\mathrm{HCl} \mathrm{pH} \mathrm{7.4,}$ $100 \mathrm{mM} \mathrm{NaCl}, 1.5 \mathrm{mM} \mathrm{MgCl}_{2}, 0.2 \%$ Triton X-100 and $1 \mathrm{mM}$ DTT. After washing steps to remove unbound protein, IgG sepharose and ZZ-UTP14A-His 10 -IgG sepharose was incubated with $200 \mathrm{pmol} \mathrm{DHX} 37-\mathrm{His}_{6}$ for $1 \mathrm{~h}$ rotating at $4{ }^{\circ} \mathrm{C}$. The beads were then washed and elution was performed by treatment with $2 \mu \mathrm{M}$ TEV protease overnight at $4{ }^{\circ} \mathrm{C}$ in 50 $\mathrm{mM}$ Tris- $\mathrm{HCl} \mathrm{pH} 7.4,100 \mathrm{mM} \mathrm{NaCl}, 1.5 \mathrm{mM} \mathrm{MgCl}, 0.2 \%$ Triton X-100 and $1 \mathrm{mM}$ DTT. Inputs and eluates were separated by SDS-PAGE then analysed by western blotting using antibodies listed in Supplementary Table 3.

Hydrolysis of ATP was monitored in an NADH-coupled assay. ${ }^{60}$ Reactions were set up containing $45 \mathrm{mM}$ Tris- $\mathrm{HCl} \mathrm{pH}$ 7.4, $25 \mathrm{mM} \mathrm{NaCl}, 2 \mathrm{mM} \mathrm{MgCl}, 1 \mathrm{mM}$ phosphoenolpyruvate, $300 \mu \mathrm{M} \mathrm{NADH}, 20 \mathrm{U} / \mathrm{ml}$ pyruvate kinase and $4 \mathrm{mM}$ ATP and were supplemented with 500 nM recombinant DHX37/DHX37т282/DHX15, $500 \mathrm{nM}$ recombinant UTP14A and/or $1.5 \mu \mathrm{M}$ RNA (5'-GUAAUGUAAGUGAACGUAAAACAAAACAAAAC-3') as indicated. The decrease in absorbance at $340 \mathrm{~nm}$ was monitored using a BioTEK Synergy plate reader and this was used to calculate the rate of ATP hydrolysis. 


\section{ACKNOWLEDGEMENTS}

We would like to thank Jens Kretschmer for help with bioinformatics analyses and cloning, and Claudia Schneider for helpful discussions. This work was funded by the Deutsche Forschungsgemeinschaft (SFB1190 and BO3442/1-2) and the University Medical Centre Göttingen.

\section{DISCLOSURE OF INTEREST}

The authors declare no conflict of interest. 


\section{REFERENCES}

1. Anger AM, Armache J-P, Berninghausen $O$, Habeck M, Subklewe M, Wilson DN, Beckmann R. Structures of the human and Drosophila 80 S ribosome. Nature 2013; 497:80-5.

2. Woolford JLJ, Baserga SJ. Ribosome biogenesis in the yeast Saccharomyces cerevisiae. Genetics 2013; 195:643-81.

3. Andersen JS, Lyon CE, Fox AH, Leung AKL, Lam YW, Steen H, Mann M, Lamond Al. Directed proteomic analysis of the human nucleolus. Curr Biol 2002; 12:1-11.

4. Scherl A, Coute Y, Deon C, Calle A, Kindbeiter K, Sanchez J-C, Greco A, Hochstrasser D, Diaz J-J. Functional proteomic analysis of human nucleolus. Mol Biol Cell 2002; 13:4100-9.

5. Wild T, Horvath P, Wyler E, Widmann B, Badertscher L, Zemp I, Kozak K, Csucs G, Lund $E$, Kutay $U$. A protein inventory of human ribosome biogenesis reveals an essential function of exportin 5 in 60S subunit export. PLoS Biol 2010; 8:e1000522.

6. Tafforeau L, Zorbas C, Langhendries J-L, Mullineux S-T, Stamatopoulou V, Mullier R, Wacheul L, Lafontaine DLJ. The complexity of human ribosome biogenesis revealed by systematic nucleolar screening of Pre-rRNA processing factors. Mol Cell 2013; 51:539-51.

7. Badertscher L, Wild T, Montellese C, Alexander LT, Bammert L, Sarazova M, Stebler M, Csucs G, Mayer TU, Zamboni N, et al. Genome-wide RNAi Screening Identifies Protein Modules Required for 40S Subunit Synthesis in Human Cells. Cell Rep 2015; 13:2879-91.

8. Farley-Barnes KI, McCann KL, Ogawa LM, Merkel J, Surovtseva Y V, Baserga SJ. Diverse Regulators of Human Ribosome Biogenesis Discovered by Changes in Nucleolar Number. Cell Rep 2018; 22:1923-34.

9. Sloan KE, Bohnsack MT, Schneider C, Watkins NJ. The roles of SSU processome components and surveillance factors in the initial processing of human ribosomal RNA. RNA 2014; 20:540-50. 
10. Sloan KE, Mattijssen S, Lebaron S, Tollervey D, Pruijn GJM, Watkins NJ. Both endonucleolytic and exonucleolytic cleavage mediate ITS1 removal during human ribosomal RNA processing. J Cell Biol 2013; 200:577-88.

11. Preti M, O'Donohue M-F, Montel-Lehry N, Bortolin-Cavaille M-L, Choesmel V, Gleizes P-E. Gradual processing of the ITS1 from the nucleolus to the cytoplasm during synthesis of the human 18S rRNA. Nucleic Acids Res 2013; 41:4709-23.

12. Mills EW, Green R. Ribosomopathies: There's strength in numbers. Science 2017; 358.

13. Pelletier J, Thomas G, Volarevic S. Ribosome biogenesis in cancer: new players and therapeutic avenues. Nat Rev Cancer 2018; 18:51-63.

14. Henras AK, Plisson-Chastang C, O'Donohue M-F, Chakraborty A, Gleizes P-E. An overview of pre-ribosomal RNA processing in eukaryotes. Wiley Interdiscip Rev RNA $2015 ; 6: 225-42$.

15. Watkins NJ, Bohnsack MT. The box C/D and H/ACA snoRNPs: key players in the modification, processing and the dynamic folding of ribosomal RNA. Wiley Interdiscip Rev RNA 2012; 3:397-414.

16. Sloan KE, Warda AS, Sharma S, Entian K-D, Lafontaine DLJ, Bohnsack MT. Tuning the ribosome: The influence of rRNA modification on eukaryotic ribosome biogenesis and function. RNA Biol 2017; 14:1138-52.

17. Wells GR, Weichmann F, Colvin D, Sloan KE, Kudla G, Tollervey D, Watkins NJ, Schneider C. The PIN domain endonuclease Utp24 cleaves pre-ribosomal RNA at two coupled sites in yeast and humans. Nucleic Acids Res 2016; 44:5399-409.

18. Wells GR, Weichmann F, Sloan KE, Colvin D, Watkins NJ, Schneider C. The ribosome biogenesis factor yUtp23/hUTP23 coordinates key interactions in the yeast and human pre-40S particle and hUTP23 contains an essential PIN domain. Nucleic Acids Res 2017; 45:4796-809.

19. Warda AS, Freytag B, Haag S, Sloan KE, Gorlich D, Bohnsack MT. Effects of the Bowen-Conradi syndrome mutation in EMG1 on its nuclear import, stability and nucleolar recruitment. Hum Mol Genet 2016; 25:5353-64. 
20. Kass S, Tyc K, Steitz JA, Sollner-Webb B. The U3 small nucleolar ribonucleoprotein functions in the first step of preribosomal RNA processing. Cell 1990; 60:897-908.

21. Sharma K, Venema J, Tollervey D. The 5' end of the 18S rRNA can be positioned from within the mature rRNA. RNA 1999; 5:678-86.

22. Hughes JM. Functional base-pairing interaction between highly conserved elements of U3 small nucleolar RNA and the small ribosomal subunit RNA. J Mol Biol 1996; 259:645-54.

23. Borovjagin A $\vee$, Gerbi SA. The spacing between functional Cis-elements of U3 snoRNA is critical for rRNA processing. J Mol Biol 2000; 300:57-74.

24. Barandun J, Chaker-Margot M, Hunziker M, Molloy KR, Chait BT, Klinge S. The complete structure of the small-subunit processome. Nat Struct Mol Biol 2017; 24:944-53.

25. Cheng J, Kellner N, Berninghausen O, Hurt E, Beckmann R. 3.2-A-resolution structure of the $90 \mathrm{~S}$ preribosome before A1 pre-rRNA cleavage. Nat Struct Mol Biol $2017 ; 24: 954-64$.

26. Sardana R, Liu X, Granneman S, Zhu J, Gill M, Papoulas O, Marcotte EM, Tollervey D, Correll CC, Johnson AW. The DEAH-box helicase Dhr1 dissociates U3 from the pre-rRNA to promote formation of the central pseudoknot. PLoS Biol 2015; 13:e1002083.

27. Sloan KE, Gleizes P-E, Bohnsack MT. Nucleocytoplasmic Transport of RNAs and RNA-Protein Complexes. J Mol Biol 2016; 428:2040-59.

28. Linder P, Jankowsky E. From unwinding to clamping - the DEAD box RNA helicase family. Nat Rev Mol Cell Biol 2011; 12:505-16.

29. Jarmoskaite I, Russell R. RNA helicase proteins as chaperones and remodelers. Annu Rev Biochem 2014; 83:697-725.

30. Liang $\mathrm{X}-\mathrm{H}$, Fournier MJ. The helicase Has1p is required for snoRNA release from prerRNA. Mol Cell Biol 2006; 26:7437-50.

31. Bohnsack MT, Kos M, Tollervey D. Quantitative analysis of snoRNA association with pre-ribosomes and release of snR30 by Rok1 helicase. EMBO Rep 2008; 9:1230-6. 
32. Bohnsack MT, Martin R, Granneman S, Ruprecht M, Schleiff E, Tollervey D. Prp43 Bound at Different Sites on the Pre-rRNA Performs Distinct Functions in Ribosome Synthesis. Mol Cell 2009; 36:583-92.

33. Dembowski JA, Kuo B, Woolford JL. Has1 regulates consecutive maturation and processing steps for assembly of 605 ribosomal subunits. Nucleic Acids Res 2013; 41:7889-904.

34. Pertschy B, Schneider C, Gnadig M, Schafer T, Tollervey D, Hurt E. RNA helicase Prp43 and its co-factor Pfa1 promote 20 to $18 \mathrm{~S}$ rRNA processing catalyzed by the endonuclease Nob1. J Biol Chem 2009; 284:35079-91.

35. Martin R, Straub AU, Doebele C, Bohnsack MT. DExD/H-box RNA helicases in ribosome biogenesis. RNA Biol 2013; 10:4-18.

36. Srivastava L, Lapik YR, Wang M, Pestov DG. Mammalian DEAD box protein Ddx51 acts in 3' end maturation of 28S rRNA by promoting the release of U8 snoRNA. Mol Cell Biol 2010; 30:2947-56.

37. Kellner M, Rohrmoser M, Forne I, Voss K, Burger K, Muhl B, Gruber-Eber A, Kremmer E, Imhof A, Eick D. DEAD-box helicase DDX27 regulates 3 ' end formation of ribosomal 47S RNA and stably associates with the PeBoW-complex. Exp Cell Res 2015; 334:146-59.

38. Memet I, Doebele C, Sloan KE, Bohnsack MT. The G-patch protein NF-kappaBrepressing factor mediates the recruitment of the exonuclease XRN2 and activation of the RNA helicase DHX15 in human ribosome biogenesis. Nucleic Acids Res 2017; 45:5359-74.

39. Calo E, Flynn RA, Martin L, Spitale RC, Chang HY, Wysocka J. RNA helicase DDX21 coordinates transcription and ribosomal RNA processing. Nature 2015; 518:249-53.

40. Sloan KE, Leisegang MS, Doebele C, Ramirez AS, Simm S, Safferthal C, Kretschmer J, Schorge T, Markoutsa S, Haag S, et al. The association of late-acting snoRNPs with human pre-ribosomal complexes requires the RNA helicase DDX21. Nucleic Acids Res 2015; 43:553-64.

41. Larburu N, Montellese C, O'Donohue M-F, Kutay U, Gleizes P-E, Plisson-Chastang C. Structure of a human pre-40S particle points to a role for RACK1 in the final steps of $18 S$ rRNA processing. Nucleic Acids Res 2016; 44:8465-78. 
42. Ameismeier M, Cheng J, Berninghausen O, Beckmann R. Visualizing late states of human 40 S ribosomal subunit maturation. Nature 2018; 558:249-53.

43. Wang M, Pestov DG. 5'-end surveillance by Xrn2 acts as a shared mechanism for mammalian pre-rRNA maturation and decay. Nucleic Acids Res 2011; 39:1811-22.

44. Zorbas C, Nicolas E, Wacheul L, Huvelle E, Heurgue-Hamard V, Lafontaine DLJ. The human 18S rRNA base methyltransferases DIMT1L and WBSCR22-TRMT112 but not rRNA modification are required for ribosome biogenesis. Mol Biol Cell 2015; 26:2080-95.

45. Carron C, O'Donohue MF, Choesmel V, Faubladier M, Gleizes PE. Analysis of two human pre-ribosomal factors, bystin and hTsr1, highlights differences in evolution of ribosome biogenesis between yeast and mammals. Nucleic Acids Res 2011; 39:28091.

46. McCaughan UM, Jayachandran U, Shchepachev V, Chen ZA, Rappsilber J, Tollervey D, Cook AG. Pre-40S ribosome biogenesis factor Tsr1 is an inactive structural mimic of translational GTPases. Nat Commun 2016; 7:11789.

47. Leulliot N, Bohnsack MT, Graille M, Tollervey D, Van tilbeurgh $\mathrm{H}$. The yeast ribosome synthesis factor Emg1 is a novel member of the superfamily of alpha/beta knot fold methyltransferases. Nucleic Acids Res 2008; 36:629-39.

48. Meyer B, Wurm JP, Kotter P, Leisegang MS, Schilling V, Buchhaupt M, Held M, Bahr $\mathrm{U}$, Karas M, Heckel A, et al. The Bowen-Conradi syndrome protein Nep1 (Emg1) has a dual role in eukaryotic ribosome biogenesis, as an essential assembly factor and in the methylation of Psi1191 in yeast 18S rRNA. Nucleic Acids Res 2011; 39:1526-37.

49. Haag S, Kretschmer J, Bohnsack MT. WBSCR22/Merm1 is required for late nuclear pre-ribosomal RNA processing and mediates N7-methylation of G1639 in human $18 \mathrm{~S}$ rRNA. RNA 2015; 21:180-7.

50. Linder P, Jankowsky E. From unwinding to clamping - the DEAD box RNA helicase family. Nat Rev Mol Cell Biol 2011; 12:505-16.

51. Bohnsack MT, Tollervey D, Granneman S. Identification of RNA helicase target sites by UV cross-linking and analysis of cDNA [Internet]. 1st ed. Elsevier Inc.; 2012. Available from: http://dx.doi.org/10.1016/B978-0-12-396546-2.00013-9 
52. Haag S, Kretschmer J, Sloan KE, Bohnsack MT. Crosslinking Methods to Identify RNA Methyltransferase Targets In Vivo. Methods Mol Biol 2017; 1562:269-81.

53. Sloan KE, Bohnsack MT. Unravelling the Mechanisms of RNA Helicase Regulation. Trends Biochem Sci 2018; 43:237-50.

54. Sardana R, Zhu J, Gill M, Johnson AW. Physical and functional interaction between the methyltransferase Bud23 and the essential DEAH-box RNA helicase Ecm16. Mol Cell Biol 2014; 34:2208-20.

55. Létoquart J, Huvelle E, Wacheul L, Bourgeois G, Zorbas C, Graille M, HeurguéHamard V, Lafontaine DLJ. Structural and functional studies of Bud23-Trm112 reveal $18 S$ rRNA N7-G1575 methylation occurs on late 40 S precursor ribosomes. Proc Natl Acad Sci U S A 2014; 111:E5518-26.

56. Zhu J, Liu X, Anjos M, Correll CC, Johnson AW. Utp14 Recruits and Activates the RNA Helicase Dhr1 To Undock U3 snoRNA from the Preribosome. Mol Cell Biol 2016; 36:965-78.

57. Ounap K, Kasper L, Kurg A, Kurg R. The human WBSCR22 protein is involved in the biogenesis of the $40 S$ ribosomal subunits in mammalian cells. PLoS One 2013; 8:e75686.

58. Choque E, Schneider C, Gadal O, Dez C. Turnover of aberrant pre-40S pre-ribosomal particles is initiated by a novel endonucleolytic decay pathway. Nucleic Acids Res 2018; 46:4699-714.

59. White J, Li Z, Sardana R, Bujnicki JM, Marcotte EM, Johnson AW. Bud23 methylates G1575 of 18S rRNA and is required for efficient nuclear export of pre-40S subunits. Mol Cell Biol 2008; 28:3151-61.

60. Heininger AU, Hackert $P$, Andreou AZ, Boon K-L, Memet I, Prior M, Clancy A, Schmidt B, Urlaub $H$, Schleiff $E$, et al. Protein cofactor competition regulates the action of a multifunctional RNA helicase in different pathways. RNA Biol 2016; 13:320-30. 


\section{FIGURE LEGENDS}

Figure 1. Depletion of the nucleolar RNA helicase DHX37 affects early and late stages of 18S rRNA maturation. (A) HeLa cells were fixed and the localisation of DHX37 was determined by immunofluorescence using an antibody against endogenous DHX37 (green). Immunofluorescence against NSUN5 (red) served as a nucleolar marker and nuclear material was visualised by DAPI staining (blue). An overlay of the images is provided and a scale bar representing $10 \mu \mathrm{m}$ is depicted. (B) HeLa cells were left untransfected (WT), or were transfected with non-target siRNA (siNT) or siRNAs targeting DHX37 (siDHX37_1 and siDHX37_2). After $72 \mathrm{~h}$, cells were harvested, and proteins were analysed by western blotting using antibodies against DHX37 and tubulin. (C) Whole cell extracts from cells treated with non-target siRNAs (siNT) or a siRNA targeting DHX37 (siDHX37_1) were separated by sucrose density gradient centrifugation. The absorbance of each fraction at $260 \mathrm{~nm}$ was used to generated a profile on which the positions of the ribosomal and preribosomal complexes are indicated. (D) Schematic view of the major pre-rRNA intermediates detected in human cells. Mature rRNA regions are shown as black rectangles, and internal (ITS) and external transcribed spacer (ETS) regions are represented by black lines. Cleavage sites relevant for maturation of the $18 \mathrm{~S}$ rRNA are named above the $47 \mathrm{~S}$ rRNA transcript and the hybridisation position of the 5' ITS1 probe used for northern blotting is indicated by the triangle. (E) Untransfected HeLa cells, or HeLa cells that had been treated with non-target siRNAs (siNT) or those targeting DHX37 (siDHX37_1) for $72 \mathrm{~h}$ were starved of phosphate then grown in media supplemented with $\left[{ }^{32} \mathrm{P}\right]$-orthophosphate for $1 \mathrm{~h}$ before growth in normal DMEM for $3 \mathrm{~h}$. Total RNA was extracted, separated by denaturing agarose gel electrophoresis, transferred to a nylon membrane and nascent RNAs were detected using a phosphorimager. The mature 28S rRNA was visualised using methylene blue staining (MB). (F) Total RNA was extracted from wild-type (WT) HeLa cells or HeLa cells transfected with siRNAs as in (B). RNAs were separated by denaturing agarose gel electrophoresis, transferred to a nylon membrane and the mature 28S rRNA was visualised by methylene blue staining (MB). Northern blotting with a probe hybridising to the 5 ' end of ITS1 (5' ITS1) was used to detect precursors of the 18S RNA, which were visualised using a phosphorimager. Aberrant pre-rRNA species detected upon depletion of DHX37 are indicated by arrows. Experiments were performed at least in triplicate and representative data are shown.

Figure 2. Mapping of the aberrant $16 \mathrm{~S}^{*}$ and $38 \mathrm{~S}^{*}$ pre-rRNA intermediates that accumulate upon depletion of DHX37. (A) Schematic view of the $47 \mathrm{~S}$ pre-rRNAs (black) and the aberrant $38 S^{*}, 16 S^{*}$ and $30 S^{2} 5$ ' pre-rRNA species not normally detected in human cells 
(grey). Mature rRNA regions are shown as rectangles, and internal (ITS) and external transcribed spacer (ETS) regions are represented by lines. Cleavage sites are named above the 47S pre-rRNA and the hybridisation position of probes used for northern blotting are indicated. (B,C) RNA extracted from HeLa cells transfected with non-target siRNAs (siNT) or siRNAs targeting DHX37 (siDHX37_1) was separated by denaturing agarose gel electrophoresis, transferred to a nylon membrane and the mature 28S rRNA was detected by methylene blue staining (MB). Northern blotting was performed using probes hybridising to different positions within the 5' ETS and ITS1 (B) or the 18S rRNA (C) and pre-rRNAs were visualised using a phosphorimager. (D) RNA extracted from wildtype (WT) HeLa cells or cells that had been transfected with non-target siRNAs (siNT), or siRNAs targeting DHX37 (siDHX37_1) or XRN2 (siXRN2) was analysed as in (B, C) using a probe hybridising to the 5' end of ITS1. Experiments were performed at least in triplicate and representative data are shown.

Figure 3. Pre-rRNAs are degraded upon depletion of DHX37 and expression of catalytically inactive DHX37 leads to defects in $A^{\prime}$ cleavage and the conversion of $21 \mathrm{~S}$ to $18 \mathrm{SE}$. (A) Cterminally His 6 -tagged $\mathrm{DHX} 37$ or $\mathrm{DHX} 37$ carrying a threonine to alanine substitution at amino acid 282 within the evolutionarily conserved "GKT" motif (DHX37T282A) was recombinantly expressed in E. coli and purified. Purified proteins were separated by SDSPAGE and visualised by Coomassie staining. (B) The amount of ATP hydrolysed by recombinant DHX37 or DHX37T282A in the presence $\left(^{+}\right)$or absence $(-)$of RNA was determined using an in vitro NADH-coupled ATPase assay. Experiments were performed in triplicate and error bars represent mean \pm standard deviation. (C) HEK293 cell lines were transfected with either non-target siRNAs (siNT) or siRNAs targeting DHX37 (siDHX37_1) and expression of the Flag tag, or C-terminally Flag-tagged DHX37 or DHX37 induced by addition of tetracycline $24 \mathrm{~h}$ before harvesting. Proteins were extracted and analysed by western blotting using antibodies against DHX37, tubulin and the Flag tag. (D) Total RNA extracted form HEK293 cell lines treated as described in (C) was separated by denaturing agarose gel electrophoresis, transferred to a nylon membrane and analysed by northern blotting using probes hybridising at the 5' end of ITS1 (left panel) or within the 5' ETS (right panel). Pre-rRNAs were detected using a phosphorimager and the mature $28 \mathrm{~S}$ rRNA was visualised by methylene blue staining (MB).

Figure 4. DHX37 associates with the 3 ' region of the U3 snoRNA that contains the box C/D motifs. (A) HEK293 cells expressing DHX37-Flag or the Flag tag were grown in the presence of 4-thiouridine before crosslinking in vivo using light at $365 \mathrm{~nm}$. RNA-protein complexes were tandem affinity purified under native and denaturing conditions, then RNAs co-purified with DHX37 were ligated to sequencing adaptors and labelled using $\left[{ }^{32} \mathrm{P}\right]$. Complexes were separated by denaturing PAGE, transferred to a nitrocellulose membrane 
and labelled RNAs were detected by autoradiography. The regions of the membrane excised for subsequent analysis are indicated by boxes. (B) The region of the membrane containing DHX37-Flag-RNA complexes as indicated in (A), and a corresponding region of the lane containing the Flag sample, were excised. RNAs were isolated and subjected to reverse transcription and PCR amplification to generate a cDNA library upon which Illumina deep sequencing was performed. The obtained sequence reads were mapped to the human genome and, after normalisation, the relative number of reads derived from snoRNAs in the Flag and DHX37-Flag samples was determined. (C) The relative distribution of sequence reads derived from each box C/D snoRNA in the Flag and DHX37Flag datasets is shown. The proportion of sequence reads mapping to the U 3 snoRNA is highlighted in red while all other snoRNAs are shown in shades of grey. (D) Cell extracts prepared from HEK293 cells expressing Flag-DHX37 or the Flag tag were incubated with anti-Flag beads. After thorough washing steps, complexes were eluted and RNA was extracted. RNAs extracted from inputs $(1 \%)$ and eluates were separated by denaturing PAGE, transferred to a nylon membrane and northern blotting was performed using probes hybridising to the U3 and U8 snoRNAs. RNAs were detected using a phosphorimager. (E) The normalised number of reads mapping to each nucleotide of the gene encoding the U3 snoRNA in the Flag (black) and DHX37-Flag (red) datasets is shown graphically (upper panel). The normalised number of mutations mapping to each position are indicated in the lower panel. A schematic representation of the U3 snoRNA is shown below with the relative positions of key features indicated.

Figure 5. The U3 snoRNA accumulates on pre-ribosomes upon expression of catalytically inactive DHX37. (A) HEK293 cells capable of expression of DHX37-Flag or DHX37 T282AFlag were transfected with siRNAs against DHX37 and $24 \mathrm{~h}$ prior to harvesting, expression of the tagged proteins was induced by addition of tetracycline. To confirm equal expression levels, proteins were analysed by western blotting using antibodies against DHX37 and tubulin. (B) Whole cell extracts prepared from HEK293 cells treated as in (A) were separated by sucrose density gradient centrifugation. The optical density of each faction at $260 \mathrm{~nm}$ was determined and used to generate a profile on which the peaks corresponding to ribosomal and pre-ribosomal complexes are indicated. RNA extracted from the gradient fractions described in (B) was separated by denaturing PAGE and transferred to a nylon membrane. Northern blotting was performed using a $\left[{ }^{32} \mathrm{P}\right]$-labelled probe hybridising to the U3 snoRNA. The experiments shown in this figure were performed in triplicate and representative data are shown.

Figure 6. UTP14A interacts with DHX37 and stimulates its ATPase activity in vitro. (A) Cell extracts prepared from HEK293 cells expressing either DHX37-Flag or the Flag tag were used for immunoprecipitation experiments in the presence (+) or absence (-) of RNase. 
Inputs $(1 \%)$ and eluates were separated by SDS-PAGE and analysed by western blotting using antibodies against DHX37, UTP14A, WBSCR22 and tubulin. (B) Recombinant DHX37-His 6 was incubated with either IgG sepharose (beads) or with IgG sepharose on which ZZ-UTP14A-His ${ }_{10}$ had been immobilised. After washing steps, proteins were eluted, and inputs and eluates were separated by SDS-PAGE then analysed by western blotting using antibodies against DHX37 and UTP14A. (C) The amount of ATP hydrolysed by recombinant $\mathrm{DHX} 37$ in the presence $(+)$ or absence $(-)$ of RNA and the presence $(+)$ or absence (-) of UTP14A was determined using an in vitro NADH-coupled ATPase assay. Experiments were performed in triplicate and error bars represent mean \pm standard deviation. (D) HeLa cells were left untransfected (WT), or were transfected with non-target siRNA (siNT) or siRNAs targeting UTP14A (siUTP14A_1 and siUTP14A_2). After $72 \mathrm{~h}$, cells were harvested, and proteins were analysed by western blotting using antibodies against UTP14A (upper panel) and tubulin (lower panel). (E) Total RNA was extracted from wildtype (WT) HeLa cells or HeLa cells transfected with siRNAs as in (D). RNAs were separated by denaturing agarose gel electrophoresis, transferred to a nylon membrane and the mature $28 \mathrm{~S}$ rRNA was visualised by methylene blue staining (MB). Northern blotting with a probe hybridising to the 5' end of ITS1 (5' ITS1) was used to detect precursors of the 18S RNA, which were visualised using a phosphorimager. (F) Whole cell extracts prepared from HEK293 cells treated with control siRNAs (siNT) or siRNAs targeting UTP14A (siUTP14A_1) were separated by sucrose density gradient centrifugation. The absorbance of each faction at $260 \mathrm{~nm}$ was determined and used to generate a profile on which the peaks corresponding to ribosomal subunits (40S and 60S) and 80S monosomes are indicated. RNA extracted from the gradient fractions was separated by denaturing PAGE, transferred to a nylon membrane and northern blotting was performed using a $\left[{ }^{32} \mathrm{P}\right]$-labelled probe hybridising to the U3 snoRNA. Replica experiments have been performed and representative data is shown in this figure. 


\section{SUPPLEMENTARY MATERIAL}

\section{The human RNA helicase DHX37 is required for release of the U3 snoRNP from pre- ribosomal particles}

Priyanka Choudhury, Philipp Hackert, Indira Memet, Katherine E. Sloan and Markus T. Bohnsack

\section{SUPPLEMENTARY FIGURES}

Supplementary Figure 1. Depletion of DHX37, DIMT1L or TSR1 leads to accumulation of the aberrant $16 S^{*}$ and $38 S^{*}$ pre-rRNA intermediates. (A) Secondary structure of the human mature $18 \mathrm{~S}$ rRNA with the positions of northern blot probes and the $5^{\prime}$ end of the $38 S^{*}$ prerRNA intermediate indicated. (B) HeLa cells were transfected with non-target siRNAs (siNT), siRNAs against DHX37 (siDHX37_1 or siDHX37_2), DIMT1L (siDIMT1L_1 or siDIMT1L_2) or TSR1 (siTSR1_1 or siTSR1_2) or were left untransfected (WT) and harvested after $72 \mathrm{~h}$. Proteins were separated by SDS-PAGE, transferred to a nitrocellulose membrane and analysed by western blotting using antibodies against DHX37, DIMT1L, TSR1 and, as a loading control, tubulin. (C) Total RNA extracted from siRNA-treated cells as described in $(A)$ was separated by denaturing agarose gel electrophoresis, transferred to a nylon membrane and analysed by northern blotting using a probe that hybridises to the 5 ' end of ITS1. The mature $28 \mathrm{~S}$ rRNA was visualised by methylene blue staining (MB).

Supplementary Figure 2. The distribution of DHX37 between pre-ribosomal and nonribosomal complexes. Cell extracts prepared from HeLa cells were separated by sucrose density gradient centrifugation. The absorbance of each fraction at $260 \mathrm{~nm}$ was used to generate a profile on which the positions of the $40 \mathrm{~S}$ and $60 \mathrm{~S}$ subunits, and the $80 \mathrm{~S}$ monosomes are indicated (upper panel). The distribution of DHX37 was determined by western blotting using an antibody against the endogenous protein (lower panel).

Supplementary Figure 3. Sequence alignment of yeast Dhr1 and human DHX37. An alignment of the amino acid sequences of S. cerevisiae Dhr1 (Uniprot: Q04217) and $H$. sapien DHX37 (Uniprot: Q8IY37) is shown. Amino acids numbers are indicated to the left and right and the regions of Dhr1 that are known to form protein-protein interactions are indicated.

Supplementary Figure 4. DIMT1L and TSR1 co-precipitate with DHX37 from human cells but these interactions are RNA-dependent. (A, B) Whole cell extracts prepared from HEK293 cells expressing DHX37-Flag or the Flag tag were used for immunoprecipitation experiments on anti-Flag beads in the presence $(+)$ or absence $(-)$ of RNase treatment. 
Inputs $(1 \%)$ and eluates were separated by SDS-PAGE, transferred to a nitrocellulose membrane and analysed by western blotting using antibodies against DIMT1L (A), TSR1 (B) and tubulin (A and B).

Supplementary Figure 5. UTP14A does not stimulate ATP hydrolysis by the DEAH box RNA helicase DHX15. The amount of ATP hydrolysed by recombinant DHX15 in the presence (+) or absence (-) of RNA and the presence (+) or absence (-) of UTP14A was determined using an in vitro NADH-coupled ATPase assay. Experiments were performed in triplicate and error bars represent mean \pm standard deviation. 
SUPPLEMENTARY TABLES

Supplementary Table 1. Normalised number of DHX37 CRAC sequencing reads mapping to snoRNAs

\begin{tabular}{|c|c|c|c|}
\hline Box C/D snoRNA & Hits/million & $\begin{array}{ll}\text { Box } & \text { H/ACA } \\
\text { SnoRNA } & \\
\end{array}$ & Hits/million \\
\hline SNORD1 & 20 & SNORA1 & 10 \\
\hline SNORD3 & 7645 & SNORA2 & 10 \\
\hline SNORD4 & 41 & SNORA3 & 10 \\
\hline SNORD5 & 248 & SNORA7 & 51 \\
\hline SNORD6 & 20 & SNORA10 & 20 \\
\hline SNORD7 & 20 & SNORA13 & 20 \\
\hline SNORD11 & 72 & SNORA19 & 10 \\
\hline SNORD12 & 10 & SNORA20 & 20 \\
\hline SNORD13 & 10 & SNORA22 & 10 \\
\hline SNORD14 & 61 & SNORA23 & 10 \\
\hline SNORD15 & 123 & SNORA28 & 10 \\
\hline SNORD18 & 102 & SNORA30 & 30 \\
\hline SNORD19 & 20 & SNORA31 & 20 \\
\hline SNORD20 & 62 & SNORA33 & 31 \\
\hline SNORD24 & 113 & SNORA36 & 20 \\
\hline SNORD26 & 82 & SNORA38 & 20 \\
\hline SNORD32 & 20 & SNORA41 & 10 \\
\hline SNORD34 & 41 & SNORA42 & 10 \\
\hline SNORD35 & 31 & SNORA45 & 10 \\
\hline SNORD36 & 154 & SNORA46 & 10 \\
\hline SNORD38 & 82 & SNORA48 & 20 \\
\hline SNORD42 & 51 & SNORA50 & 20 \\
\hline SNORD43 & 548 & SNORA51 & 20 \\
\hline SNORD45 & 61 & SNORA53 & 10 \\
\hline SNORD46 & 51 & SNORA55 & 20 \\
\hline SNORD47 & 10 & SNORA58 & 10 \\
\hline SNORD48 & 155 & SNORA63 & 20 \\
\hline SNORD49 & 41 & SNORA64 & 20 \\
\hline SNORD52 & 10 & SNORA66 & 20 \\
\hline SNORD53 & 51 & SNORA67 & 72 \\
\hline SNORD54 & 10 & SNORA68 & 10 \\
\hline SNORD55 & 20 & SNORA69 & 41 \\
\hline SNORD58 & 102 & SNORA70 & 30 \\
\hline SNORD59 & 31 & SNORA71 & 10 \\
\hline SNORD61 & 20 & SNORA73 & 10 \\
\hline SNORD62 & 41 & SNORA76 & 41 \\
\hline SNORD63 & 31 & SNORA78 & 10 \\
\hline SNORD66 & 41 & SNORA80 & 10 \\
\hline SNORD68 & 20 & SNORA81 & 10 \\
\hline SNORD69 & 20 & & \\
\hline SNORD70 & 20 & & \\
\hline SNORD71 & 51 & & \\
\hline
\end{tabular}




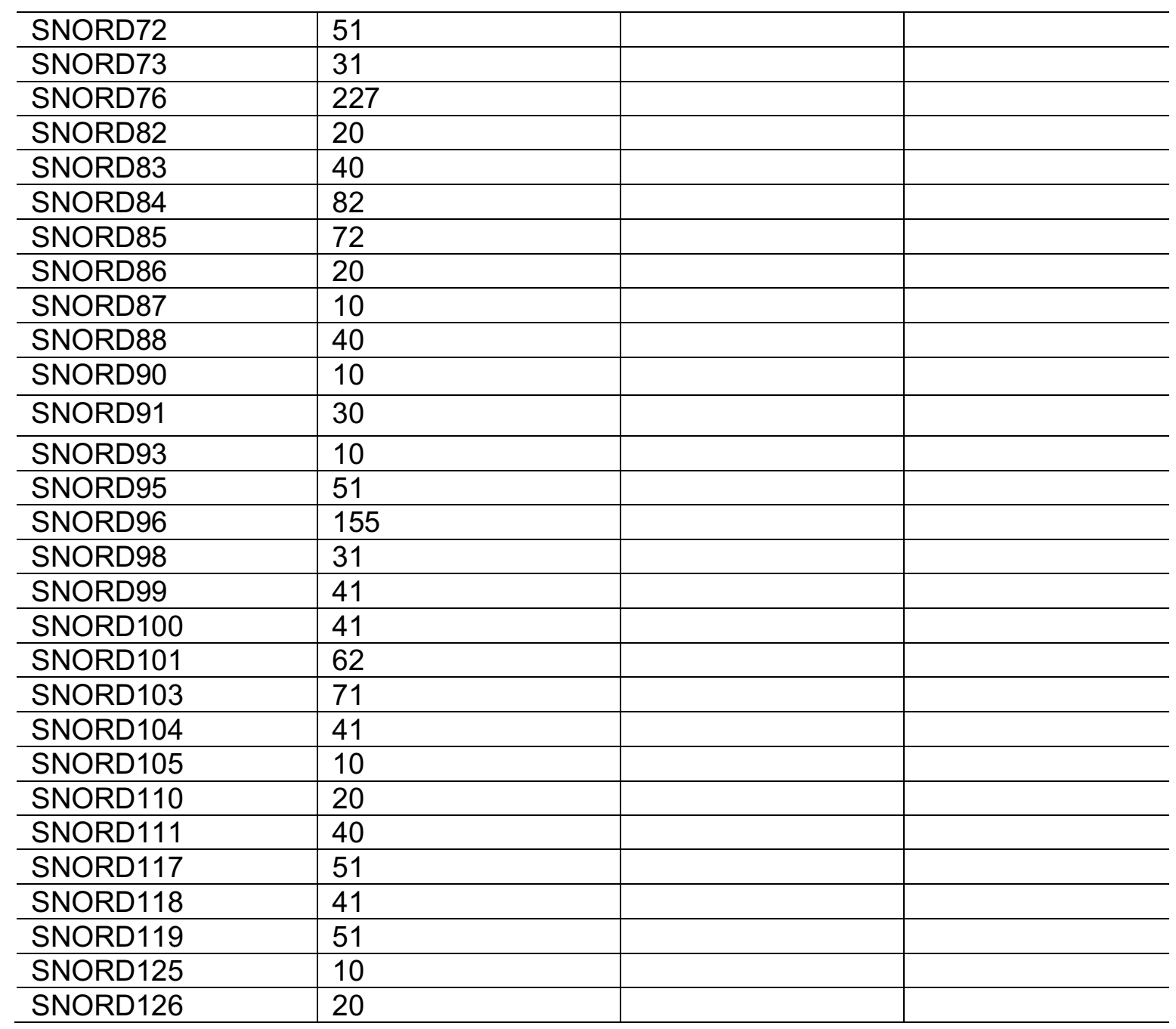

Supplementary Table 2. Sequences of siRNA used in this study

\begin{tabular}{l|l|l}
\hline Name & siRNA target sequence (5'-3') & Reference \\
\hline siNT & CGTACGCGGAATACTTCGA & 61 \\
\hline siDHX37_1 & AGAUCAACUUGGAUCAUUATT & Qiagen \\
\hline siDHX37_2 & GGAAGGUCAAGAAAACGCUATT & Qiagen \\
\hline siXRN2_1 & GGGAAGAAAUAUUGGCAA & 62 \\
\hline siDIMT1L_1 & GGCUAGUAGCUGAACUUCA & 44 \\
\hline siDIMT1L_2 & GGAUGGUCUAGUAAGGAUA & 44 \\
\hline siTSR1_1 & GGAGAUUUGUGCUACGGAU & 6 \\
\hline siTSR1_2 & GCAUCUUGCUUUUCGAGAU & 6 \\
\hline SiUTP14A_1 & CAGGAAGAACUAGCGGAUU & 63 \\
\hline siUTP14A_2 & GACGCCGGUUUCUCAUUAA & 63 \\
\hline
\end{tabular}


Supplementary Table 3. Antibodies used in this study

\begin{tabular}{l|l}
\hline Name & Source \\
\hline Anti-Flag & Sigma F3165 \\
\hline Anti-DHX37 & Bethyl A300-857A \\
\hline Anti-WBSCR22 & Abgent AP20254b \\
\hline Anti-NSUN5 & Santa Cruz Sc-376147 \\
\hline Anti-DIMT1L & Sigma HP042944 \\
\hline Anti-TSR1 & Proteintech 16887-1-AP \\
\hline Anti-UTP14A & Proteintech 11474-1-AP \\
\hline Anti-WBSCR22 & Abgent AP20254b \\
\hline
\end{tabular}

Supplementary Table 4. Sequences of oligonucleotides used as northern blot probes in this study

\begin{tabular}{|c|c|}
\hline Name & Sequence (5'-3') \\
\hline U3 & $\begin{array}{l}\text { GCTCTACACGTTCAGAGAAACTTCTCTAGTAACACACTATAGAAATGATC } \\
\text { CC }\end{array}$ \\
\hline U8 & $\begin{array}{l}\text { GTTCTAATCTGCCCTCCGGAAGGAGGAAACAGGAAACAGGTAAGGATTA } \\
\text { TCCCACCC }\end{array}$ \\
\hline 5' ETS-A & $\begin{array}{l}\text { CGGAGGCCCAACCTCTCCGACGACAGGTCGCCAGAGGACAGCGTGTCA } \\
\text { GC }\end{array}$ \\
\hline 5' ITS1 & CCTCGCCCTCCGGGCTCCGTTAATGATC \\
\hline 5' ETS-B & GGTAAGGTAGAGCGCGGCGAGCC \\
\hline ITS1-A & AGGGGTCTTTAAACCTCCGCGCCGGAACGCGCTAGGTAC \\
\hline ITS1-B & GAGTCCGCGGTGGAG \\
\hline ITS1-C & GGTTGCCTCAGGCCG \\
\hline 18A_1 & CTGGCAGGATCAACCAGGTA \\
\hline 18S_1004 & ATTAATGAAAACATTCTTGGCA \\
\hline 18S_1230 & GTTGAGTCAAATTAAGCCGCAGG \\
\hline 18S_1285 & AGCTATCAATCTGTCAATCC \\
\hline 18S_1377 & CATGCCAGAGTCTCGTTCGTTA \\
\hline 18S_1670 & CAAAGGGCAGGGACTTAATCAACG \\
\hline
\end{tabular}




\section{SUPPLEMENTARY REFERENCES}

61. Elbashir SM, Harborth J, Weber K, Tuschl T. Analysis of gene function in somatic mammalian cells using small interfering RNAs. Methods 2002; 26:199-213.

62. West S, Gromak N, Proudfoot NJ. Human $5^{\prime}-->3^{\prime}$ exonuclease Xrn2 promotes transcription termination at co-transcriptional cleavage sites. Nature 2004 ; 432:522525.

63. Hu L, Wang J, Liu Y, Zhang Y, Zhang L, Kong R, Zheng Z, Du X, Ke Y. A small ribosomal subunit (SSU) processome component, the human U3 protein 14A (hUTP14A) binds p53 and promotes p53 degradation. J Biol Chem 2011; 286:311928. 

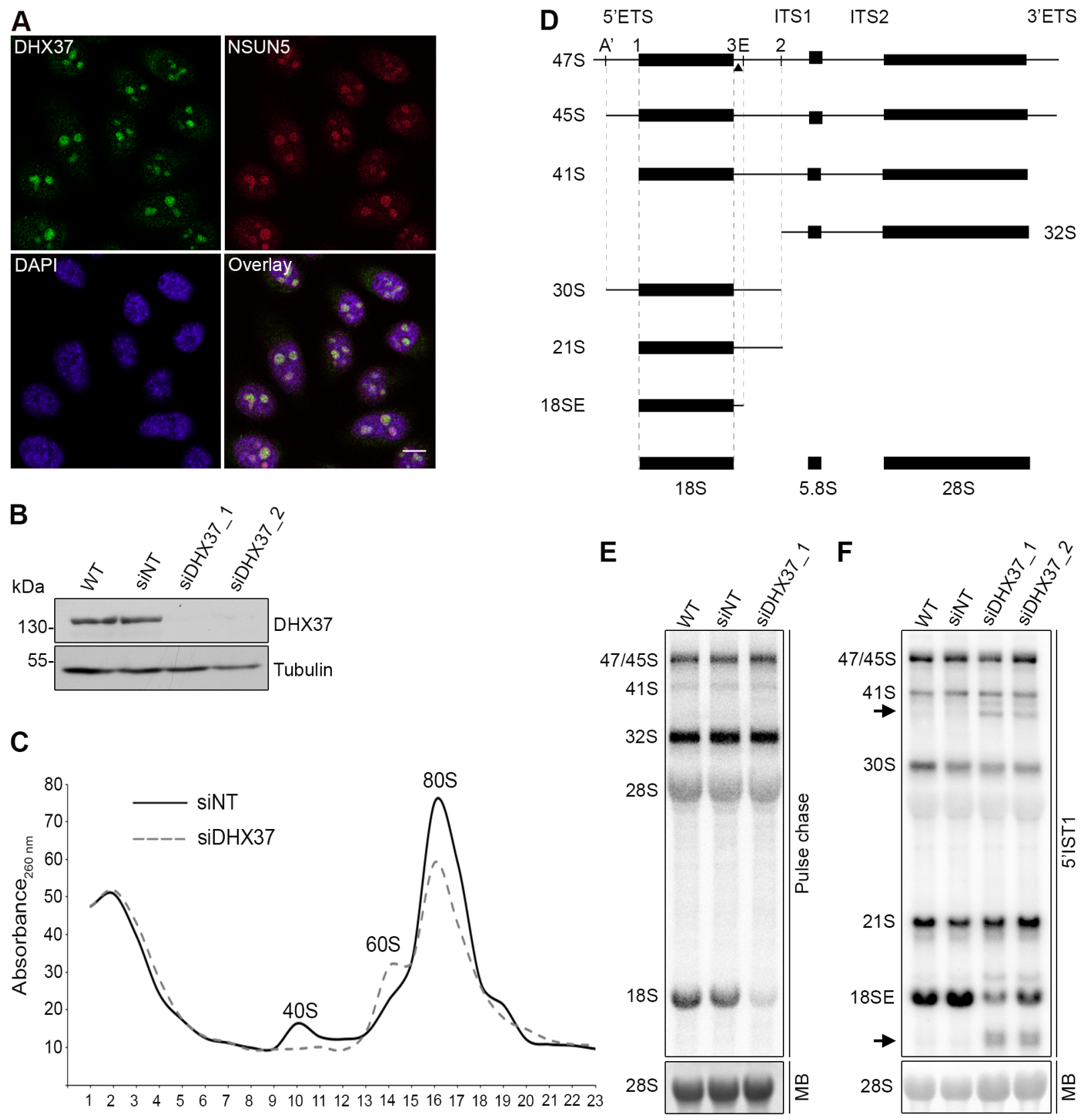

Choudhury et al., Figure 1 

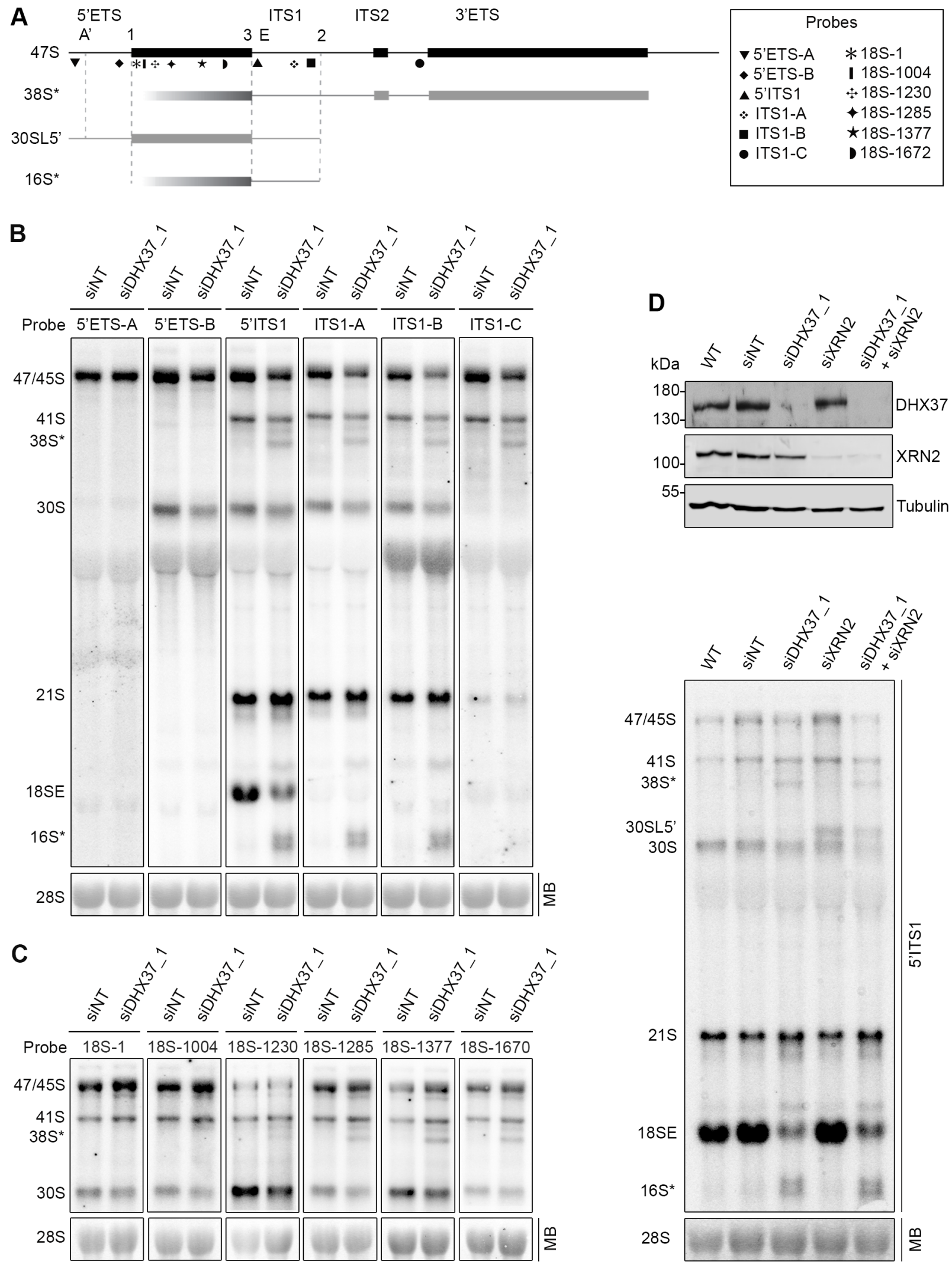

Choudhury et al., Figure 2 

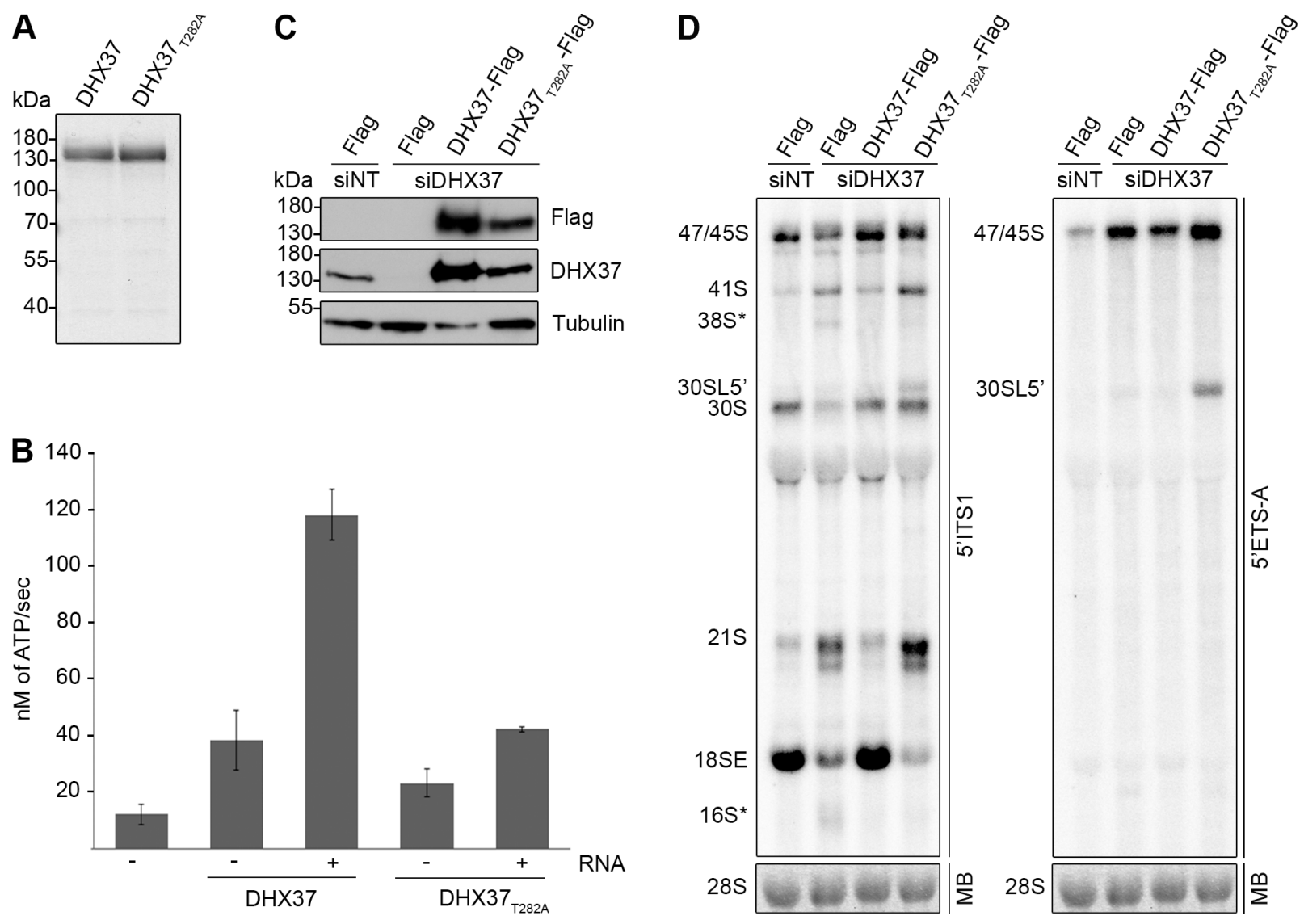

Choudhury et al., Figure 3 


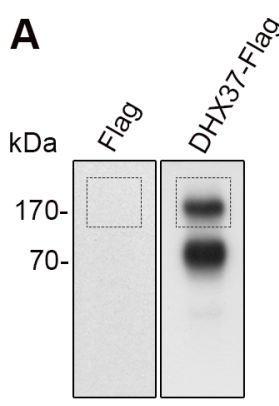

C

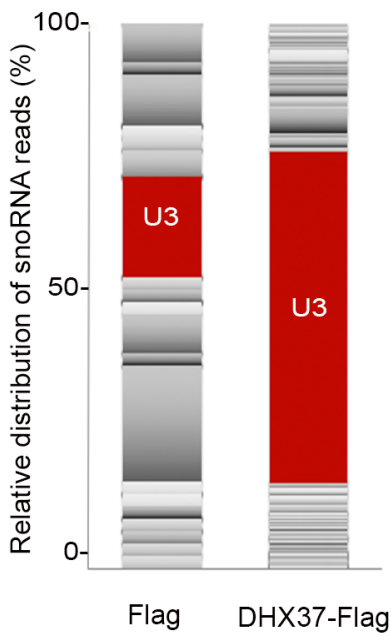

B 10-

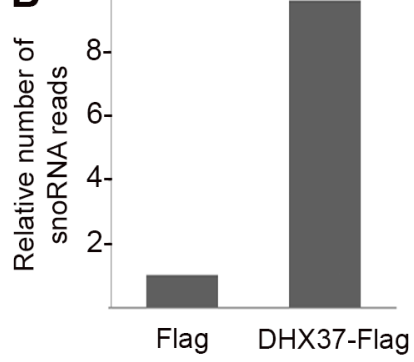

E

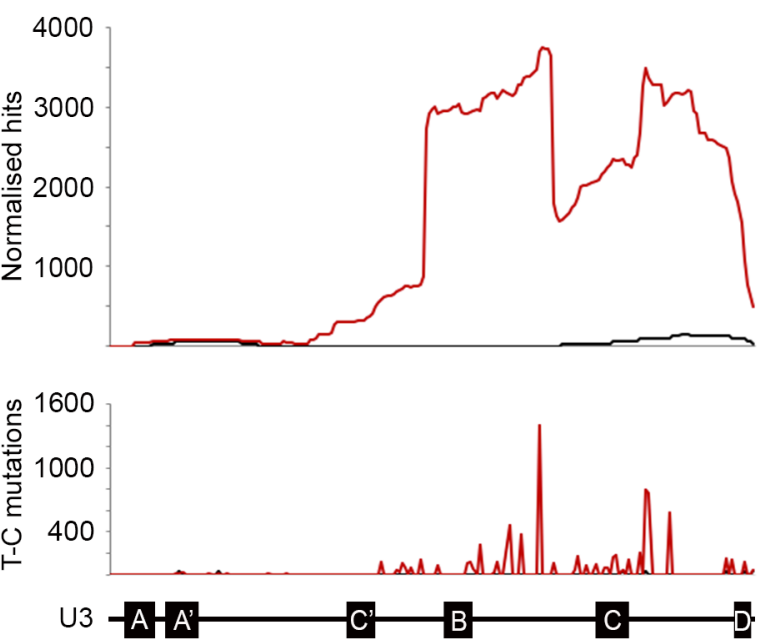

Choudhury et al., Figure 4 

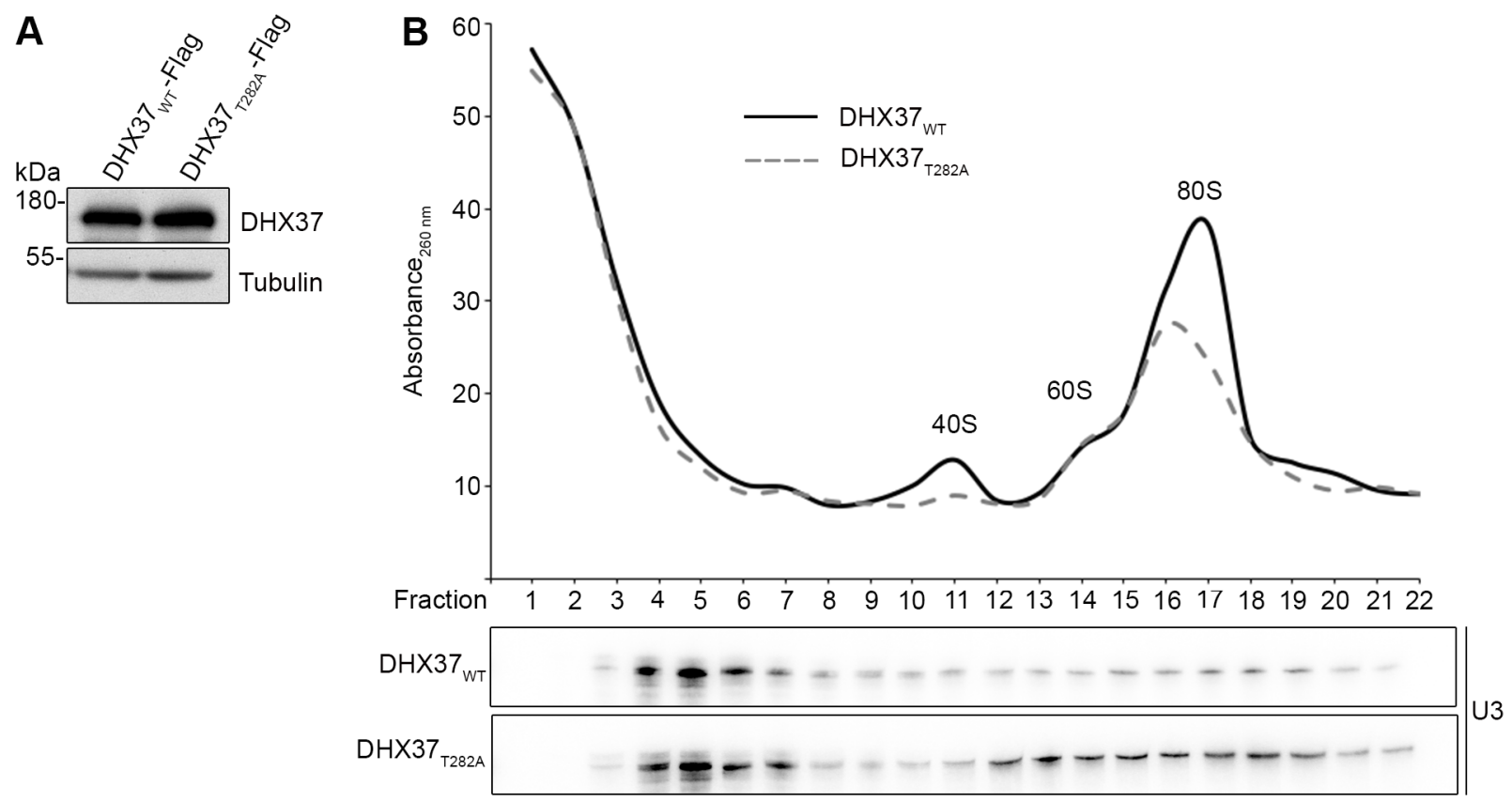

Choudhury et al., Figure 5 

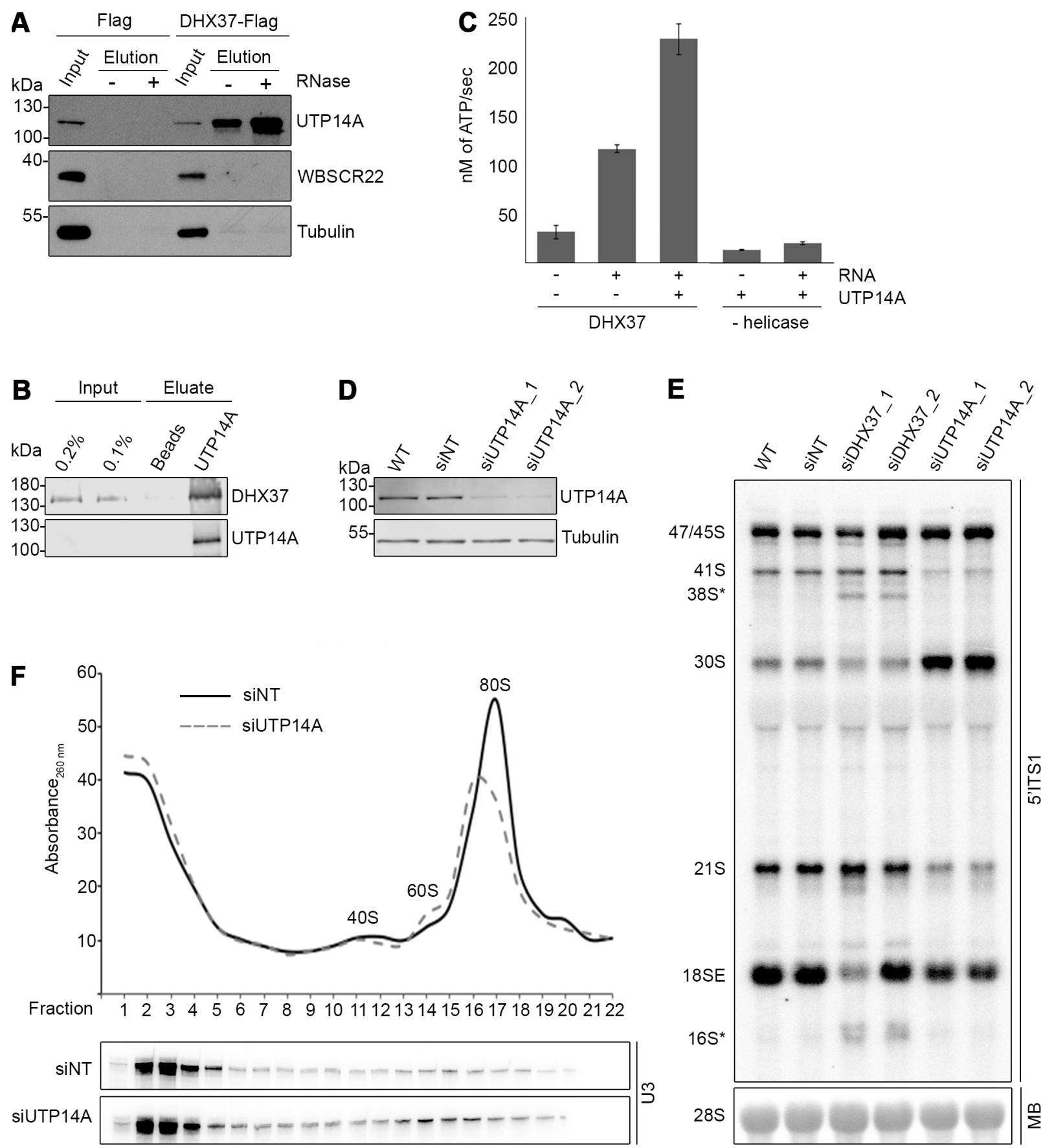

Choudhury et al., Figure 6 

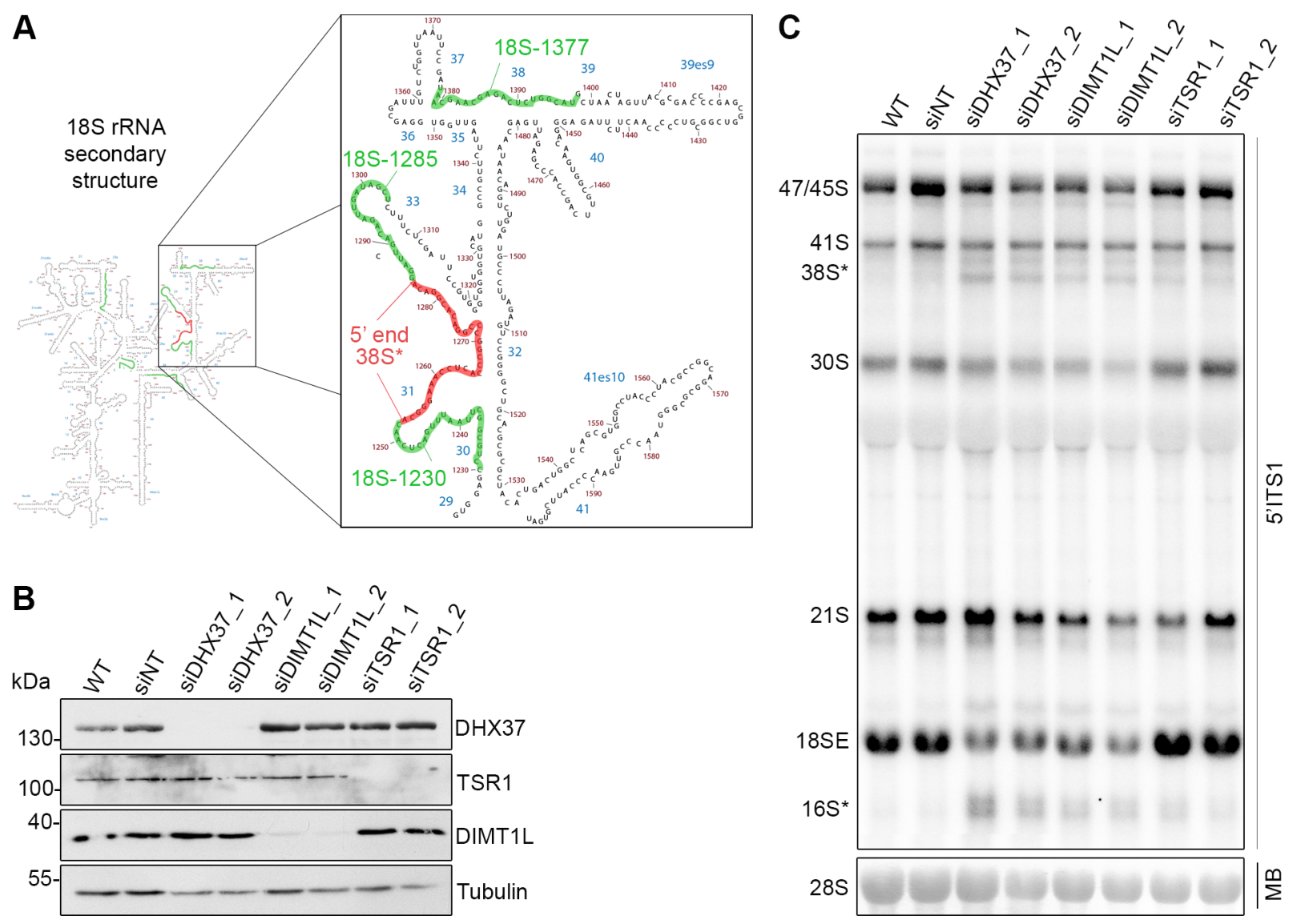

Choudhury et al., Supplementary Figure 1 


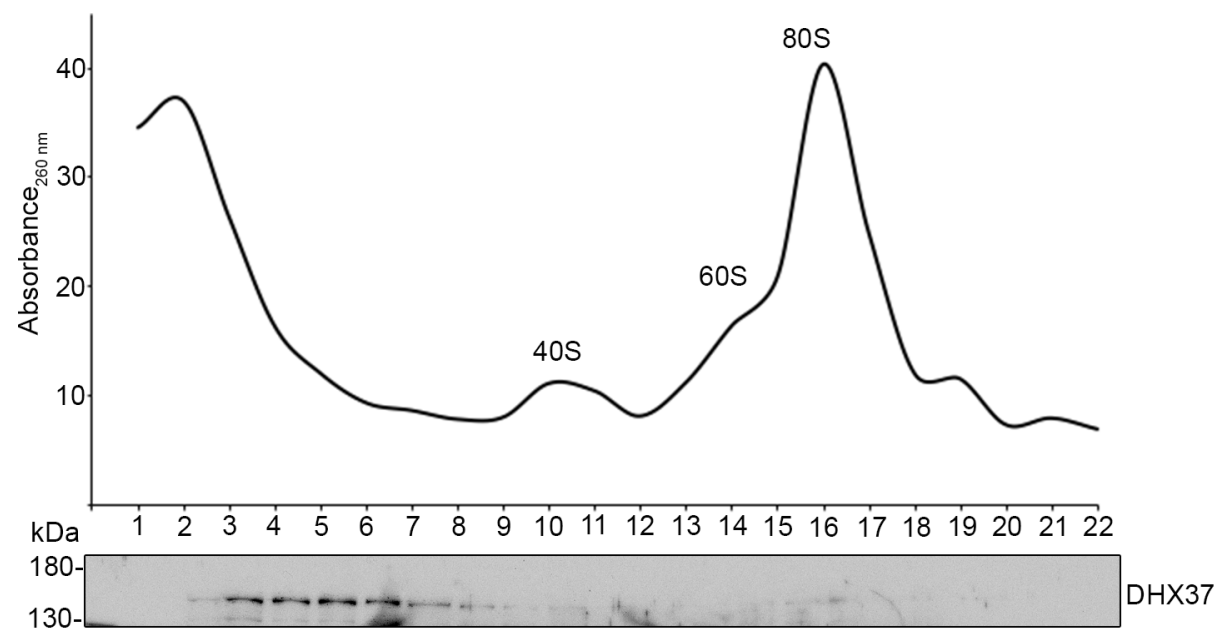

Choudhury et al., Supplementary Figure 2 
S. cerevisiae Dhr1

H. sapien DHX37

s. cerevisiae Dhr1

H. sapien DHX37

S. cerevisiae Dhr

H. sapien DHX37

S. cerevisiae Dhr1

H. sapien DHX37

S. cerevisiae Dhr

H. sapien DHX37

S. cerevisiae Dhr1

H. sapien DHX37

S. cerevisiae Dhr1

H. sapien DHX37

S. cerevisiae Dhrl

H. sapien DHX37

S. Cerevisiae Dhr1

H. sapien DHX37

S. cerevisiae Dhr1

H. sapien DHX37

S. Cerevisiae Dhr1

H. sapien DHX37

S. cerevisiae Dhrl

H. sapien DHX37

s. cerevisiae Dhr1

H. sapien DHX37

S. cerevisiae Dhrl

H. sapien DHX37

s. cerevisiae Dhr1

H. sapien DHX37

S. cerevisiae Dhr1

H. sapien DHX37
MGTYRKRFNEKARSGHMAKLKELKRIRNKQFTRQDENDERVENPDSAPAESSTTEPN--ANAE ILEPLTEEEKKMKKRKLQELFTPKESKV MGKLRRRYNIKGROOAGP----------------G---PSKGPPEPPPVQLELEDKDTLKGVDASNALVL PGKKKKKTKAPPLSKKEKKPL

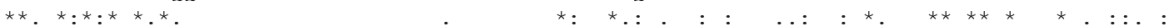
SRLKKKRLDKFIEHQLKREERKTI IGKLQDYK---IDTSLLTSSKRLGEGRQTKKEEFKEALSLERQGRGNEQTNEILYEEYEPKVWDE

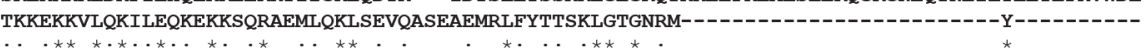

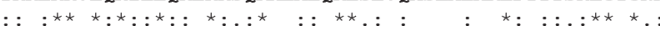

YGEGGSSEDDDGEDDFEASFGSMPKPTDNEEKKSSGFIDHRPAKFGGSGLSFGFSNIKVINKESKTPKKKYNWRQRVEMEELKKHGKEDE -------HTK-----------------E-KADEV-----------VAPGQEK---ISSLSGAHRKRRRWPSAEEEEEEEE---ESE ${ }^{*}: . . \quad:: * . \quad * \ldots *::^{*}: *^{*} . * * *::{ }^{*} *^{*}$

MDFDTTSEDDDEEEDQEEEDKMHPSENPLEEVESADSETGSEKFDQNDVANEFKDWANQEIKKLEGRDQELVTPTLNIDYKPIIRKEDLD

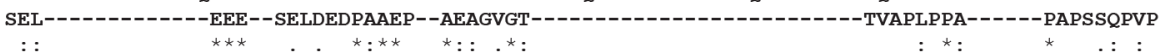

DGL-----QEAYVPINENSTRKAFYVEVSRSDE IQKARIQLPVFGEEHKIMEAIHHNDVVI ICGETGSGKTTQVPQFLYEAGFGAEDSPD AGMTVPPPPAAAPPLPRALAKPAVFI PVNRSPEMQEERLKLPILSEEQVIMEAVAEHPIVIVCGETGSGKTTQVPQFLYEAGFSSEDS--

YPGMVGITQPRRVAAVSMAERVANELGDHGHKVGYQIRFDSTAKEDTKVKFMTDGVLLREMMHDFKLTKYSS IIIDEAHERNINTDILIG --IIGVTEPRRVAAVAMSQRVAKEMNLSQRVVSYIRYEGNVTEETRIKFMTDGVLLKEIQKDFLLLRYKVVIIDEAHERSVYTDILIG

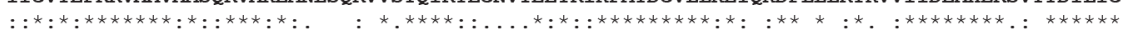

MLSRCVRLRAKLHKENPIEHKKLKLI IMSATLRVSDFSENKTLFPIAPPVLQVDARQFPVSIHF'NRRTAF-NYTDEAFRKTCKIHQKLPP LLSRIVTLRAKR-------NLPLKLLTMSATLRVEDFTONPRLFAKPPPVIKVESRQFPVTVHFNKRTPLEDYSGECFRKVCKIHRMLPA

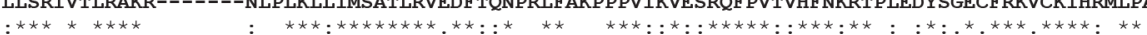

GAILVFLTGQQEITHMVKRLRKEFPFKKNSKYNKDLETP--VSKMGINSKTTDLEAEDIDFSVQVIDQDKFKSAIRYEEDEGNSGNGEDE GGILVFLTGQAEVHALCRRLRKAFPPSRARPQEKDDDQKDSVEEMRKFKKSRARAKKARAEVLPQINLDHYSVLP---AGEGDED-REAE

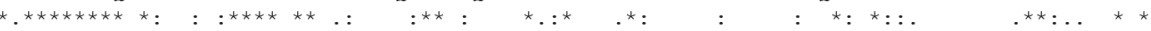

EDEEEEGFE----EVL--------TEGQTANDPLYVLPLYSLLPTKEQMRVFQKPPQGSRLCIVATNVAETSLTIPGVRYVVDSGRSKERK VDEEEGALDSDLDLDLGDGGQDGGEQPDASLPLHVLPLYSLLAPEKQAOVFKPPPEGTRLCVVATNVAETSLTI PGIKYVVDCGKVKKRY

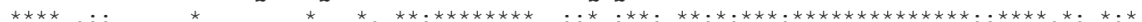

YNESNGVQSFEVGWVKASANQRSGRAGRTGPGHCYRLYSSAVFEHDFEQFSKPEILRMPVES IVLQMKSMAIHNIINFPFPTPPDRVAL YDRVTGVSSFRVTWVSQASADQRAGRAGRTEPGHCYRLYSSAVFG-DFEQFPPPEITRRPVEDLILQMKALNVEKVINFPFPTPPSVEAL

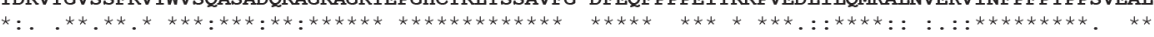

SKAIQLLQYLGALDNK----------------EMITEDGKKMSLFPLSPRFSKMLLVSDEKACLPYIVAIVSALSVGDPFINEFELGINEI LAAEELLIALGALQPPQKAERVKQLQENRLSCPITALGRTMATFPVAPRYAKMLALSRQHGCLPYAITIVASMTVRELFEEL-

$* \cdot * * \quad * * * *$ :

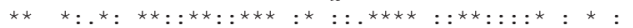

SRKPNPDENLDDKIREHDESTPGMDPELKKELRSKFYKSRSQF SKLDKFSDVFRLLSVVSAMDYVPKEQKEIFMKKNFLRGKLMEEIVKL DRPAASDEELTR-LKSKRARVAQM---------K-RTW---AGQGASLKLGDLMVLLGAVGACEYASCT--PQFCEANGLRYKAMME IRRL

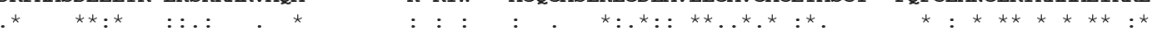
RKQLMY IIKSNTSKENIAVVIRNEDLKSDIPSVIQIKLLKQMICAGFVDHVAVRADVL--FPDDAKITNRTSIINIPYI PVLATRTPNIE RGQLTTAVNAVCPEAELF------VDPKMQPPTESQVTYLRQIVTAGLGDHLARRVQSEEMLEDKWRNAYKT----------------P-LL

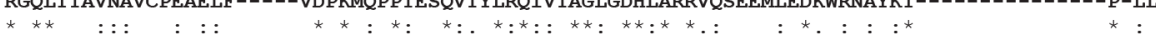
DCFVYIHPTS ILNNLGEMPPKYMLYYSLHLGGNNKTRMNTLCDIASTPLANIARKGLLLTYSKPLTGQGLKTVNLSPTERYCYVVPRF-G DDPVFIHPSSVLFK---ELPEFVVYQEIVET--TKMYMKGVSSVEVQWIPAL--LPSYCQFDKPLE---------EPAPTYCPERGRVLC

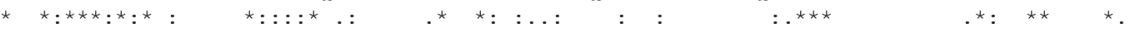

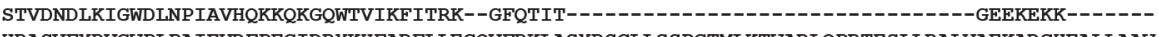
HRASVFYRVGWPLPAIEVDFPEGIDRYKHFARFLLEGQVFRKLASYRSCLLSSPGTMLKTWARLQPRTESLLRALVAEKADCHEALLAAW ${ }^{*}:$ : :

1267

KKNPKYLLAEYCEWLPQAMHPDIEKAWPPTTVH 1154

\section{Choudhury et al., Supplementary Figure 3}



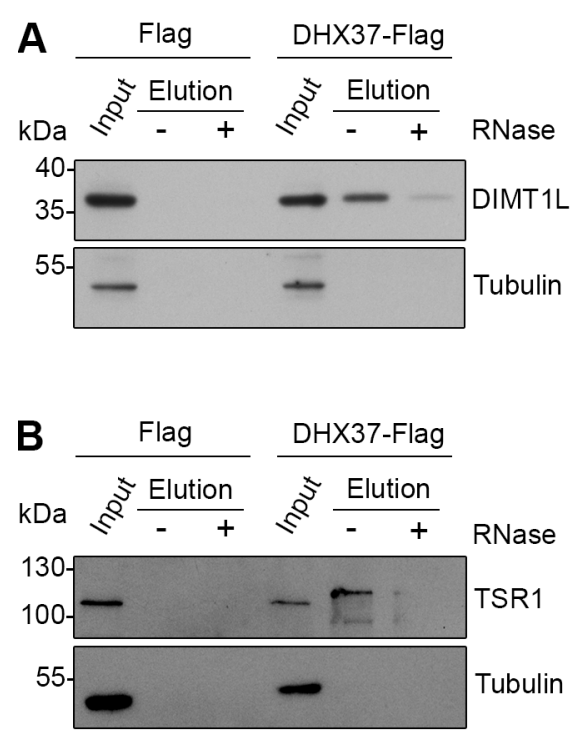

Choudhury et al., Supplementary Figure 4 


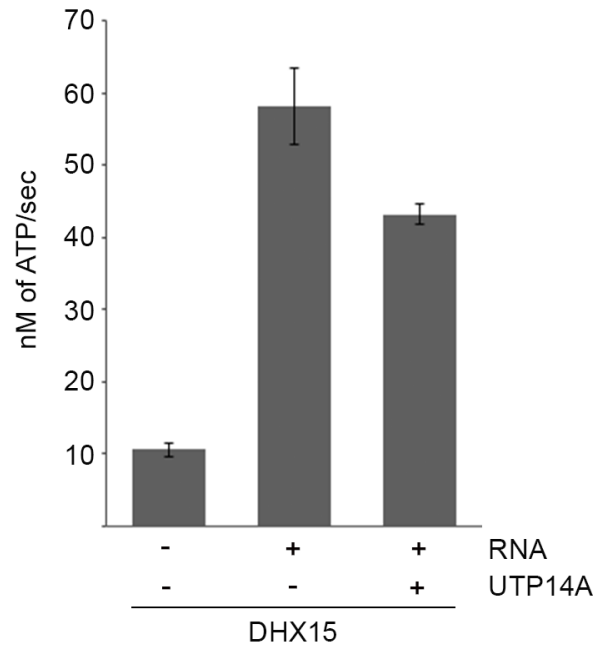

Choudhury et al., Supplementary Figure 5 


\section{Chapter Four:}

\section{The role of DDX55 in human ribosome biogenesis}

\subsection{Introduction}

The eukaryotic ribosome is a large ribonucleoprotein complex, composed of the large and small subunits, $60 \mathrm{~S}$ and $40 \mathrm{~S}$, respectively, which associate in the cytoplasm to form translation-competent $80 \mathrm{~S}$ ribosomal complexes (Klinge et al., 2012). Ribosome biogenesis is one of the most dynamic and energy consuming processes in the cell with the production of approximately 2000 ribosomes per minute in yeast (Warner, 2001). In mammalian cells, owing to a higher degree of complexity, the number is significantly greater with the ribosome assembly machinery manufacturing approximately 7500 ribosomal subunits per minute (Lewis and Tollervey, 2000). In humans, ribosome assembly initiates in the nucleolus with the RNA polymerase I-mediated synthesis of the $47 \mathrm{~S}$ pre-rRNA transcript. The primary transcript associates with a subset of ribosomal proteins and trans-acting ribosome assembly factors to form $90 \mathrm{~S}$ pre-ribosomal complexes. An early cleavage within the prerRNA separates the precursors of the small and the large ribosomal subunits, which undergo independent maturation steps assisted by a diverse range of factors, for example, ATPase, GTPases, small nucleolar RNAs (snoRNAs) and RNA helicases before nuclear export (Anger et al., 2013; Ben-Shem et al., 2011). In yeast, more than 200 trans-acting factors have been identified, which associate with pre-ribosomal complexes and mediate important maturation events such as pre-rRNA folding, processing and structural remodelling (Woolford and Baserga, 2013). As the pathway of ribosomal assembly is conserved to a large extent amongst eukaryotes, homologues of known yeast biogenesis factors are generally expected to play similar roles in humans (Abdelhaleem et al., 2003). Although this appears to be true for a subset of biogenesis factors, a pioneering microscopybased screen highlighted interesting differences in the assembly pathways of human and yeast ribosomal subunits (Wild et al., 2010). Furthermore, analysis of pre-rRNA processing defects due to RNAi-mediated depletion of putative ribosomal assembly factors identified 74 assembly factors in humans that lack homologues in yeast (Tafforeau et al., 2013). Proteomic analysis of nucleolar morphology and number have further contributed to the growing list of assembly factors in humans with no known yeast homologues (Ahmad et al., 2009; Andersen et al., 2002; Farley-Barnes et al., 2018). Therefore, the emergence of novel biogenesis factors in humans having additional or different roles than their yeast counterparts necessitates detailed characterization of the human ribosome assembly pathway. 
RNA helicases are RNA-dependent NTPases that play key roles in mediating structural remodelling events during ribosome production (Martin et al., 2013). In yeast, 21 RNA helicases are known to associate transiently with pre-ribosomal complexes of which several (e.g. Prp43, Has1, Rok1, Dhr1) have been implicated in modulating snoRNA association with pre-ribosomal intermediates (Bohnsack et al., 2008, 2009; Martin et al., 2013; Sardana et al., 2015). Additionally, others (e.g. Prp43, Has1, Spb4, Mak5) have been shown to facilitate incorporation of ribosomal proteins/biogenesis factors and promote structural remodelling of pre-ribosomal subunits (Brüning et al., 2018; Dembowski et al., 2013; Pertschy et al., 2009). Detailed molecular characterization of several yeast RNA helicases has revealed that they fulfil similar roles in humans. For example, in humans, DHX37 (Dhr1) and SK2L2 (Mtr4) perform similar functions as their yeast counterparts (Choudhury et al., 2019; Schilders et al., 2007). However, RNA helicases have emerged to play additional or novel roles during human ribosome biogenesis. For instance, DHX15, in humans, has been implicated in the processing of metazoan-specific A' site (Memet et al., 2017) but does not seem be required for late-stage processing events of 18S rRNA (Sloan et al., 2019). In contrast, its yeast homologue Prp43, in addition to its role in snoRNA release during pre$60 \mathrm{~S}$ biogenesis, is involved in the cytoplasmic maturation phase of the 18S rRNA (Pertschy et al., 2009). Additionally, in humans, the DEAD-box helicase DDX51 is implicated in the release of U8 snoRNA from pre-rRNA subunits. Notably, DDX51 displays a high degree of homology with yeast DEAD-box protein Dbp6, although there is no yeast counterpart of U8 snoRNA suggesting that DDX51 might have acquired additional roles in higher eukaryotes (Srivastava et al., 2010). Likewise, the DEAD-box helicase elF4AIll and its yeast orthologue Fal1, are implicated in SSU biogenesis. Moreover, in humans, elF4AIII is also a major component of the EJC suggesting the acquirement of additional metazoan-specific function in the context of pre-mRNA splicing (Alexandrov et al., 2011). Recent reports have provided insights into the role of RNA helicases such as DDX21 with no known yeast homologues (Sloan et al., 2015). Genome-wide screens have suggested that RNAi-mediated depletion of several RNA helicases lead to impaired pre-rRNA processing during ribosome biogenesis (Tafforeau et al., 2013), however, the precise functions of many of these helicases are yet to be established.

In yeast, the DEAD-box helicase Spb4 associates with precursors of the large ribosomal subunit, promoting structural re-arrangement and has been implicated in the association of large subunit export adaptor, Arx1 (Brüning et al., 2018). Based on its homology to Spb4, DDX55 is anticipated to play a key role in ribosome biogenesis in humans, however, the precise role of DDX55 remains to be elucidated. Although the process of ribosome biogenesis is generally conserved amongst eukaryotes, growing evidence has highlighted 
the different and/or additional roles of RNA helicases during biogenesis. Here, we aim to characterize DDX55 in humans and understand its function during ribosome assembly. 


\subsection{Material and Methods}

\subsubsection{Chemicals}

Chemicals used in this study were purchased from Carl Roth $\mathrm{GmbH}$ \& Co., AppliChem $\mathrm{GmbH}$ and Sigma-Aldrich Chemie $\mathrm{GmbH}$ unless stated otherwise.

All enzymes were purchased from Thermo Fischer Scientific or New England Biolabs. DNA and RNA oligonucleotides were supplied by Sigma-Aldrich and IDT, respectively.

\subsubsection{Plasmids}

Table 1 Plasmids used in this study

\begin{tabular}{l|l|l|l|l}
\hline ID & Construct & CDS & Vector & Source \\
\hline p1698 & ZZ-DDX55-His 7 & NM_020936.3 & pQE80 (A15) & This study \\
\hline p1700 & ZZ-DDX55 T60A-His $_{7}$ & NM_020936.3 & pQE80 (A15) & This study \\
\hline p1699 & $\begin{array}{l}\text { ZZ-DDX55 } \\
\text { His-term- }\end{array}$ & NM_020936.3 & pQE80 (A15) & This study \\
\hline p1019 & DDX55-His 6 -2xFlag & NM_020936.3 & pcDNA5/FRT/TO & Bohnsack lab \\
\hline p1702 & $\begin{array}{l}\text { DDX55 } \\
\text { 2x-term-His } 6^{-}\end{array}$ & NM_020936.3 & pcDNA5/FRT/TO & This study \\
\hline p187 & His6-2xFlag & - & pcDNA5/FRT/TO & Bohnsack lab \\
\hline
\end{tabular}

\subsubsection{Oligonucleotides}

Table 2 Oligonucleotides used in this study

\begin{tabular}{|c|c|c|}
\hline Name/Constructs & Forward/ reverse primer sequence $\left(5^{\prime}-3^{\prime}\right)$ & Application \\
\hline \multirow{2}{*}{ ZZ-DDX55-His7 } & ATATATTCATGAGAATGGAGCATGTGACAGAGG & \multirow{2}{*}{ Cloning } \\
\hline & ATATATGGATCCGCAGTCATCTTCCAAATCTG & \\
\hline \multirow{2}{*}{$\begin{array}{l}\text { ZZ- DDX55 }{ }_{\Delta C \text {-term- }} \\
\text { His }_{7}\end{array}$} & ATATATTCATGAGAATGGAGCATGTGACAGAGG & \multirow{2}{*}{ Cloning } \\
\hline & ATATATGGATCCGTCCGCTGTGTTTCTCTGGG & \\
\hline \multirow{2}{*}{ ZZ-DDX55T60A-His 7} & $\begin{array}{l}\text { CGGTCACAGGTAGTGGCAAAGCGCTCGCTTTTG } \\
\text { TCATCC }\end{array}$ & \multirow{2}{*}{$\begin{array}{l}\text { Site- } \\
\text { directed- } \\
\text { mutagenesis }\end{array}$} \\
\hline & $\begin{array}{l}\text { GATGACAAAAGCGAGCGCTTTGCCACTACCTGT } \\
\text { GACCG }\end{array}$ & \\
\hline \multirow{2}{*}{$\begin{array}{l}\text { DDX55 }{ }_{\Delta \mathrm{C} \text {-term-His }}{ }^{-} \\
\text {2xFlag }\end{array}$} & ATATGGATCCACCATGGAGCATGTGACAGAGG & \multirow{2}{*}{ Cloning } \\
\hline & ATATGGCTAGCGCAGTCATCTTCCAAATCTG & \\
\hline
\end{tabular}




\subsubsection{Small Interfering RNAs (siRNAs)}

Table 3 siRNAs used in this study

\begin{tabular}{l|l|l}
\hline Name & Sense sequence (5'-3') & Reference \\
\hline siNT (non-target) & UCGUAAGUAAGCGCAACCC(dTdT) & Elbashir et al., 2001 \\
\hline DDX55_1 & CAGUCUUGGAGUAGUCUAA(dTdT) & Tafforeau et al., 2013 \\
\hline DDX55_2 & GGAGUUCCGCAAAUUGCAA(dTdT) & Tafforeau et al., 2013 \\
\hline DDX55_3 & CCGACACGAUUCCAUUUAA (dTdT) & Qiagen \\
\hline DDX55_4 & AGAGGGUUCUGAUAUUGAA(dTdT) & Qiagen \\
\hline
\end{tabular}

\subsubsection{Antibodies}

Table 4 Antibodies used in this study

\begin{tabular}{l|l}
\hline Name & Company \\
\hline Flag & Sigma-Aldrich \\
\hline Tubulin & Sigma-Aldrich \\
\hline IRDye 800 Donkey anti-rabbit & LI-COR \\
\hline IRDye 680 Donkey anti-mouse & LI-COR \\
\hline Goat-anti-mouse-Alexa594 & Jackson Immunoresearch \\
\hline Goat-anti-rabbit-Alexa488 & Jackson Immunoresearch \\
\hline
\end{tabular}




\subsubsection{Molecular Cloning}

\subsubsection{Amplification of gene of interest}

To perform molecular cloning, standard recombinant DNA procedures were followed (Sambrook and Russell, 2001). In brief, the sequence of interest was amplified from HeLa cDNA or from a pre-existing template using specific primers. The PCR reaction components and conditions are summarized in Table 5.

Table 5 PCR components and amplification protocol

\begin{tabular}{l|l}
\hline \multicolumn{2}{c}{ PCR reaction components } \\
\hline 5X HF Buffer & $10 \mu \mathrm{l}$ \\
\hline dNTPs (10 mM) & $1 \mu \mathrm{l}$ \\
\hline Template DNA & $2 \mu \mathrm{l}$ \\
\hline Forward Primer (10 mM) & $1 \mu \mathrm{l}$ \\
\hline Reverse Primer $(10 \mathrm{mM})$ & $1 \mu \mathrm{l}$ \\
\hline Phusion Polymerase & $1 \mu \mathrm{l}$ \\
\hline
\end{tabular}

\begin{tabular}{|c|c|c|}
\hline \multicolumn{3}{|c|}{ PCR amplification protocol } \\
\hline $98^{\circ} \mathrm{C}$ (denaturation) & $2 \min$ & x 1 \\
\hline $98^{\circ} \mathrm{C}$ (denaturation) & $30 \mathrm{sec}$ & \\
\hline $58^{\circ} \mathrm{C}$ (annealing) & $40 \mathrm{sec}$ & $\times 35$ \\
\hline $72^{\circ} \mathrm{C}$ (elongation) & $1 \mathrm{~min} / \mathrm{kb}$ & \\
\hline $72^{\circ} \mathrm{C}$ (elongation) & $10 \min$ & x 1 \\
\hline $4^{\circ} \mathrm{C}$ & Pause & - \\
\hline
\end{tabular}

$10 \%$ of the PCR products were loaded on a $1 \%(\mathrm{w} / \mathrm{v})$ agarose gel after resuspension in $6 \mathrm{X}$ DNA loading dye $(0.2 \%$ xylene cyanol, $0.2 \%$ bromophenol blue, $60 \%$ glycerol, $60 \mathrm{mM}$ EDTA) to obtain a final concentration of $1 \mathrm{X}$. The samples were loaded onto a $1 \%$ agarose gel containing SafeView DNA stain, and separated at $120 \mathrm{~V}$ for $45 \mathrm{~min}$ in TAE (40 mM Tris$\mathrm{HCl}, 20 \mathrm{mM}$ acetic acid, $1 \mathrm{mM}$ EDTA). After visualisation of product using UV light, if a single product was obtained, the remaining PCR product was purified with NucleoSpin Gel and PCR Clean-Up kit (Machery-Nagel) following the manufacturer's instructions.

\subsubsection{Restriction digestion and ligation}

The purified PCR product (insert) and the vector backbone $(2 \mu \mathrm{g})$ were digested in $1 \mathrm{X}$ restriction enzyme buffer using $10 \mathrm{U}$ of the appropriate restriction enzymes (Thermo Fischer Scientific), in a final volume of $20 \mu \mathrm{l}$. Reactions were carried out at $37^{\circ} \mathrm{C}$ for $2 \mathrm{~h}$. Vector dephosphorylation was performed by adding $1 \mu \mathrm{l}$ of alkaline phosphatase (Thermo Fischer Scientific) and further incubation for $20 \mathrm{~min}$ at $37^{\circ} \mathrm{C}$. The digested vector backbone and insert were gel purified using NucleoSpin Gel and PCR Clean-Up kit (Machary-Nagel) following the manufacturer's instructions. Ligation reactions were set up containing $50 \mathrm{ng}$ of vector backbone and a 5-fold molar excess of insert, together with $5 U$ of T4 DNA ligase in $1 \mathrm{X}$ ligation buffer. The reaction was carried out at $16^{\circ} \mathrm{C}$, overnight. 


\subsubsection{Transformation of chemically competent $E$. coli}

Chemically competent strains of E. coli, DH5 $\alpha$ (Thermo Fischer Scientific, amplification of plasmid DNA) or BL21 (DE3) CodonPlus-RIL (Agilent, recombinant protein expression) were used for transformation in this study. The ligation mix, or $100 \mathrm{ng}$ of plasmid DNA, was added to $50 \mu \mathrm{l}$ of chemically competent cells and incubated for $20 \mathrm{~min}$ on ice. The mixture was then incubated at $42^{\circ} \mathrm{C}$ for $1 \mathrm{~min}$ followed by incubation on ice for $3 \mathrm{~min} .800 \mu \mathrm{l}$ of Lysogeny Broth (LB) media was added to the mixture, which was further incubated for $1 \mathrm{~h}$ at $37^{\circ} \mathrm{C}$, shaking. After $1 \mathrm{~h}$, cells were pelleted by centrifugation (1,000 rcf, $\left.3 \mathrm{~min}\right)$ and resuspended in $50 \mu \mathrm{l}$ of LB media and spread on LB-agar plates containing appropriate antibiotics. In case of $\mathrm{DH} 5 \alpha$ transformed with plasmid harbouring ampicillin resistance, LBagar plates containing 100 mg/ml ampicillin were used. For BL21 (DE3) CodonPlus-RIL, LBagar plates containing $100 \mu \mathrm{g} / \mathrm{ml}$ ampicillin and $34 \mu \mathrm{g} / \mathrm{ml}$ chloramphenicol were used. Plates were incubated overnight at $37^{\circ} \mathrm{C}$.

\subsubsection{Plasmid DNA extraction and sequencing}

Single colonies from LB-agar plates were inoculated overnight in $4 \mathrm{ml} \mathrm{LB}$ media containing the appropriate antibiotic and grown at $37^{\circ} \mathrm{C}$ while shaking. The following day, cells were pelleted by centrifugation (11,000 rcf, $1 \mathrm{~min}$ ) and plasmid isolation was carried out with NucleoSpin Plasmid kit (Machery-Nagel) according to manufacturer's protocol. Isolated plasmid DNA (approx. $60 \mathrm{ng}$ ) was sent for Sanger sequencing with gene/vector-specific primers to Eurofins Genomics. The obtained results were analysed using DNASTAR Lasergene 10 SeqMan software.

\subsubsection{Human cell culture methods}

\subsubsection{Cell culture conditions}

HeLa CCL-2 (ATCC) and HEK293 FIp-In T-REx (Thermo Fisher) were cultured in 1X Dulbecco's Modified Eagle Medium (DMEM, Thermo Fischer Scientific) supplemented with $10 \%$ foetal bovine serum (FBS, Merck) and 1:100 dilution of penicillin-streptomycin (stock concentration: $5000 \mathrm{U} / \mathrm{ml}$; Thermo Fischer Scientific). Cells were incubated at $37^{\circ} \mathrm{C}$ with $5 \%$ $\mathrm{CO}_{2}$.

\subsubsection{Generation of stable cell lines}

To generate inducible stable cell lines, HEK293 Flp-In T-REx cells were seeded $(250,000$ cells/well) in a 6 well plate and two wells were utilized for each cell line. The next day, cells were transfected with a reaction mixture consisting of $1.8 \mu \mathrm{g}$ of pOG44 plasmid encoding the Flp recombinase, $0.6 \mu \mathrm{g}$ of the appropriate pcDNA5-derived construct along with $91 \mu \mathrm{l}$ Opti-MEM (Thermo Fischer) and $9 \mu$ of X-tremeGENE 9 DNA transfection reagent (Roche). 
$48 \mathrm{~h}$ post transfection, cells were subjected to selection with $82.4 \mu \mathrm{g} / \mathrm{ml}$ Hygromycin $\mathrm{B}$ (Applichem) and $10 \mu \mathrm{g} / \mathrm{ml}$ Blasticidin S Hydrochloride (Applichem). After selection, cells were induced with $1 \mu \mathrm{g} / \mathrm{ml}$ tetracycline for $24 \mathrm{~h}$, harvested using trypsin and resuspened in 4X SDS loading dye $(200 \mathrm{mM}$ Tris- $\mathrm{HCl} \mathrm{pH}$ 6.8, 8\% SDS, $50 \mathrm{mM}$ EDTA, 4\% $\beta-$ mercaptoethanol, $0.08 \%$ bromophenol blue, $40 \%$ glycerol). The whole cell lysate was analysed by SDS-PAGE and western blotting.

\subsubsection{3 siRNA transfection}

HeLa cells were transfected with appropriate siRNAs using lipofectamine RNAiMAX (Thermo Fischer Scientific). $500 \mu \mathrm{l}$ Opti-MEM was mixed with $50 \mathrm{nM}$ siRNA and $5 \mu \mathrm{l}$ lipofectamine RNAiMAX in a 6 well plate and incubated for $15 \mathrm{~min}$. This was followed by addition of 100,000 HeLa cells (96 h knockdown) diluted in antibiotic-free media into the well containing the reaction mixture. The plate was gently shaken and incubated for $96 \mathrm{~h}$ at $37^{\circ} \mathrm{C}$ with $5 \% \mathrm{CO}_{2}$.

\subsubsection{Immunofluorescence}

HEK293 stable cell lines (30,000 cells/well) were grown on cover slips coated with $0.01 \%$ poly-L-lysine. Expression of the transgene was induced by the addition of $1 \mu \mathrm{g} / \mathrm{ml}$ tetracycline for $24 \mathrm{~h}$. After induction, cells were fixed with $4 \%$ paraformaldehyde in PBS for 20 min and washed two times with PBS for $10 \mathrm{~min}$. Cells were first permeabilized using $0.1 \%$ Triton X-100 in PBS for 20 min and then blocked with PBS containing 10\% FBS (Merck) and $0.1 \%$ Triton $\mathrm{X}-100$ for $2 \mathrm{~h}$. Cells were incubated with primary antibodies (antiFlag antibody and anti-UTP14A antibody (nucleolar marker)) diluted in 10\% FBS in PBS for $2 \mathrm{~h}$. The cover slips were washed 3 times, for 10 min each with PBS and subsequently incubated with Alexa Fluor 488-conjugated and Alexa Fluor 543-conjugated secondary antibodies for $1 \mathrm{~h}$. The washing steps were repeated and the cover slips were mounted using VECTASHIELD (Vector Laboratories) containing DAPI for nuclear staining. Cells were analysed by confocal microscopy using a Nikon Eclipse Ti2 microscope.

\subsubsection{Analysis of pre-ribosomal complexes by sucrose density centrifugation}

HEK293 cells stably expressing full length DDX55 and DDX55 $\Delta$ C-term were grown to $70 \%$ confluency in $10 \mathrm{~cm}$ dishes. $24 \mathrm{~h}$ post induction with $1 \mathrm{\mu g} / \mathrm{ml}$ tetracycline, cells were harvested with trypsin and centrifuged for $3 \mathrm{~min}$ at $1000 \mathrm{rcf}$. The cell pellet was resuspended in $0.5 \mathrm{ml}$ lysis buffer (50 mM Tris- $\mathrm{HCl} \mathrm{pH} 7.4,100 \mathrm{mM} \mathrm{NaCl}, 5 \mathrm{mM} \mathrm{MgCl}_{2}$ and $1 \mathrm{mM}$ DTT) supplemented with cOmplete, EDTA-free protease inhibitor ( 1 tablet per $3 \mathrm{ml}$ lysis buffer, Roche). Cells were lysed by sonication using an amplitude of $20 \%$ ( $0.3 \mathrm{sec}$ on, $0.7 \mathrm{sec}$ off, $3 \times 20$ pulses). The lysate was cleared by centrifugation at $20,000 \mathrm{rcf}$ for $20 \mathrm{~min}$ at $4^{\circ} \mathrm{C}$ and 
$10 \%$ of the total sample was reserved as input. A $10-45 \%$ sucrose gradient was used to separate ribosomal particles based on their densities. To prepare the sucrose gradients, $10 \%(\mathrm{w} / \mathrm{v})$ sucrose solution was layered on top of $45 \%(\mathrm{w} / \mathrm{v})$ sucrose solution and mixed using a gradient master (BioComp) protocol with the following settings: angle: $82^{\circ}$, speed: 19, rotation time: $1 \mathrm{~min} 25 \mathrm{sec}$. The gradient was then allowed to rest for $1 \mathrm{~h}$ at $4^{\circ} \mathrm{C}$. Next, the cleared whole cell extract was loaded onto the prepared gradient and centrifuged at 23,500 rpm for $16 \mathrm{~h}$ at $4^{\circ} \mathrm{C}$ in a Beckman Coulter SW-40Ti rotor. After the overnight centrifugation step, gradients were fractionated into $530 \mu \mathrm{l}$ samples. RNA profiles were generated by measuring the RNA content of each fraction at $254 \mathrm{~nm}$ using a NanoDrop ND2000c (Thermo Fischer Scientific). Proteins in each fraction were precipitated by addition of TCA to a final concentration of $20 \%$. The samples were thoroughly mixed by vortexing and incubated for $20 \mathrm{~min}$ on ice followed by centrifugation at $20,000 \mathrm{rcf}$ for $20 \mathrm{~min}$ at $4^{\circ} \mathrm{C}$. The supernatants were discarded and the pellets were washed with cold acetone, air dried and resuspended in $75 \mu \mathrm{l}$ water and $25 \mu \mathrm{l}$ of 4 x SDS loading dye (8\% SDS, $200 \mathrm{mM}$ Tris$\mathrm{HCl} \mathrm{pH} 6.8,4 \%$ 2- mercaptoethanol, $50 \mathrm{mM}$ EDTA, 0.08\% bromophenol blue and 40\% glycerol).

\subsubsection{RNA-based methods}

\subsubsection{RNA extraction}

For a 6 well-plate of HeLa CCL2 or HEK293 cells, media was first removed from the wells, followed by a brief PBS wash to remove residual media. $500 \mu \mathrm{l}$ of TRI-reagent (SigmaAldrich) was added directly onto the cells and the cell suspension was pipetted several times before transferring to a $1.5 \mathrm{ml}$ tube. $100 \mu \mathrm{l}$ chloroform was added to the mix and the sample was vortexed thoroughly for 2 min then incubated for 2-3 min before centrifugation at $20,000 \mathrm{rcf}$ for $20 \mathrm{~min}$ at $4^{\circ} \mathrm{C}$. The aqueous phase containing RNA was transferred to a new tube and was mixed thoroughly with equal volume of isopropanol. The mixture was incubated for 2-3 min at room temperature before centrifugation at 20,000 rcf for $20 \mathrm{~min}$ at $4^{\circ} \mathrm{C}$ to precipitate the RNA. The precipitated RNA pellet was washed with $75 \%$ ethanol, air dried and resuspended in $20 \mathrm{ul}$ water to obtain a concentration of approximately $3 \mu \mathrm{g} / \mu \mathrm{l}$.

\subsubsection{Pulse chase metabolic labelling of newly synthesised RNA and northern blotting}

siRNA-treated cells were grown in phosphate-free DMEM for 1 hour followed by a media exchange with phosphate-free DMEM supplemented with $15 \mu \mathrm{Ci} / \mathrm{mL}\left[{ }^{32} \mathrm{P}\right]$-labelled orthophosphate. Cells were further grown for $1 \mathrm{~h}$. After incubation, the labelled media was removed, cells were washed with PBS and phosphate-containing DMEM was added into wells. Cells were harvested at specific time points (30 min, $60 \mathrm{~min}, 120 \mathrm{~min}, 180 \mathrm{~min}, 240$ 
min) and total RNA was extracted. RNA samples were mixed with 5 volumes of glyoxal loading dye (72 ml DMSO, $24 \mathrm{ml}$ glyoxal, $14.4 \mathrm{ml}$ BTPE (10X), $7.2 \mathrm{ml} 80 \%$ glycerol, $20 \mu \mathrm{g} / \mathrm{ml}$ ethidium bromide) and incubated for $1 \mathrm{~h}$ at $55^{\circ} \mathrm{C}$. The samples were loaded onto a 1.2\% agarose gel in 1X BPTE (10 mM PIPES, $30 \mathrm{mM}$ Bis-Tris, $1 \mathrm{mM}$ EDTA) and were separated for $16 \mathrm{~h}$ at $60 \mathrm{~V}$. The gel was washed with $0.1 \mathrm{M} \mathrm{NaOH}$ for $20 \mathrm{~min}$, followed by two washes of 15 min each with $0.5 \mathrm{M}$ Tris- $\mathrm{HCl} \mathrm{pH} 7.4$ and $1.5 \mathrm{M} \mathrm{NaCl}$. After a final washing step for 20 min with $6 \mathrm{X}$ SSC buffer ( $900 \mathrm{mM} \mathrm{NaCl}, 90 \mathrm{mM}$ sodium citrate), RNA is transferred to Hybond-N membrane (GE-Healthcare) by capillary blotting. After transfer, RNA samples were crosslinked to the membrane at $254 \mathrm{~nm}$ with 2 cycles of $240 \mathrm{~mJ} / \mathrm{cm} 2 \mathrm{in}$ a Stratalinker UV Crosslinker (Stratagene) and the membrane containing labelled RNAs were then exposed to a phosphorimager screen (GE-Healthcare) and signals were detected with Typhoon FLA 9500 (GE-Healthcare). Northern blotting was carried out to detect actin mRNA which served as loading control. The membrane was pre-hybridized for 40 min with SES1 (0.25 M sodium phosphate pH 7.0, 7 \% SDS, 1 mM EDTA) prior to incubation with $5^{\prime}$ $\left[{ }^{32} \mathrm{P}\right]$-labelled DNA oligonucleotides. To prepare the labelled probes, 20 pmol of DNA oligonucleotide was incubated together with $10 \cup \mathrm{T} 4$ polynucleotide kinase (Thermo Fisher Scientific) and $25 \mu \mathrm{Ci}\left[\gamma^{32} \mathrm{P}\right]-\mathrm{ATP}$ (PerkinElmer) in 1X T4 polynucleotide kinase buffer (Thermo Fisher Scientific). The mixture was incubated at $37^{\circ} \mathrm{C}$ for 30 min prior to its addition to the tube containing the membrane along with SES1. The labelled probe was incubated with the membrane overnight at $37^{\circ} \mathrm{C}$. Next, the membrane was washed first with $6 \mathrm{X} \mathrm{SSC}$ followed by $2 \mathrm{XSSC}+0.1 \%$ SDS for 30 min each at $37^{\circ} \mathrm{C}$. The dried membrane was then exposed to a phosphorimager screen and detected as above.

\subsubsection{Crosslinking and analysis of cDNA}

HEK293 stable cell lines expressing DDX55-Flag or the Flag-tag alone were induced with $1 \mu \mathrm{g} / \mathrm{ml}$ with tetracycline for $24 \mathrm{~h}$ followed by in vivo crosslinking $\left(1.5 \times 10^{8}\right.$ cells/ sample $)$ at $254 \mathrm{~nm}$ with $2400 \mathrm{~mJ} / \mathrm{cm}^{2}$. Cells were harvested by addition of $200 \mu \mathrm{l}$ of TNM150 buffer (50 $\mathrm{mM}$ Tris- $\mathrm{HCl} \mathrm{pH} 7.8,150 \mathrm{mM} \mathrm{NaCl}, 1.5 \mathrm{mM} \mathrm{MgCl} 2,5 \mathrm{mM} \beta$-mercaptoethanol, 0.1\% NP-40) supplemented with cOmplete, EDTA-free protease inhibitor (Roche) and lysed by sonication using an amplitude of $40 \%$ ( $0.5 \mathrm{sec}$ on, $0.5 \mathrm{sec}$ off, $3 \times 15$ pulses). The lysate was cleared by centrifugation at $20000 \mathrm{rcf}$ for $15 \mathrm{~min}$ at $4^{\circ} \mathrm{C}$. The cleared lysate was incubated with 50 $\mu$ of pre-equilibrated anti-Flag M2 magnetic beads (Sigma Aldrich) for $2 \mathrm{~h}$. Next, the beads were washed twice with TMN1000 buffer (50 mM Tris-HCl pH 7.8, $1000 \mathrm{mM} \mathrm{NaCl}, 1.5 \mathrm{mM}$ $\mathrm{MgCl}_{2}, 5 \mathrm{mM} \beta$-mercaptoethanol, 0.1\% NP-40) and three times with TNM150 buffer. Elution of bound RNA-protein complexes was carried out overnight at $4^{\circ} \mathrm{C}$ by addition of TMN150 buffer supplemented with $250 \mu \mathrm{g} / \mathrm{ml}$ Flag peptide (Sigma-Aldrich). Next, elutes were treated 
with $0.1 \mathrm{U}$ RNace-It (Agilent) and samples were incubated for $30 \mathrm{sec}$ at $37^{\circ} \mathrm{C}$. The samples were then supplemented with $6 \mathrm{M}$ Guanidinium hydochloride, $300 \mathrm{mM} \mathrm{NaCl}$ and10 $\mathrm{mM}$ imidazole before incubation with $100 \mu \mathrm{l}$ Ni-NTA slurry pre-equilibrated with WB1 (50 mM Tris- $\mathrm{HCl} \mathrm{pH} 7.8,300 \mathrm{mM} \mathrm{NaCl}, 0.1 \% \mathrm{NP}-40,6 \mathrm{M}$ guanidine hydrochloride, $10 \mathrm{mM}$ imidazole, $5 \mathrm{mM} \beta$-mercaptoethanol) for $2 \mathrm{~h}$ at $4^{\circ} \mathrm{C}$. The beads were washed twice with WB1 followed by three times with PNK buffer (50 mM Tris- $\mathrm{HCl} \mathrm{pH} \mathrm{7.8,} 10 \mathrm{mM} \mathrm{MgCl}$, 0.5\% NP-40, $5 \mathrm{mM} \beta$-mercaptoethanol). Dephosphorylation was carried out with $8 \mathrm{U}$ of TSAP (Promega) in PNK buffer for $30 \mathrm{~min}$ at $37^{\circ} \mathrm{C}$ and then the beads were washed once with WB1 and three times with PNK buffer. On-bead ligation of the Illumina RA3 3' adapter was carried out in PNK buffer containing $1 \mu \mathrm{M}$ RA3 3' adapter, $800 \mathrm{U}$ T4 RNA ligase II deletion mutant (Epicentre), $60 \cup$ RNasin (Promega) and $10 \%$ PEG8000 (Sigma-Aldrich) at $16^{\circ} \mathrm{C}$, overnight. The beads were washed as described before and radioactive labelling of the 5' ends of RNAs fragment was carried out. Phosphorylation was performed for $40 \mathrm{~min}$, at $37^{\circ} \mathrm{C}$ with $80 \mathrm{U}$ T4 PNK (NEB) and $40 \mu \mathrm{Ci}\left[{ }^{32} \mathrm{P}\right]-\mathrm{ATP}$, followed by addition of $1.25 \mathrm{mM} \mathrm{ATP}$-lithium salt (Roche) and incubation for an additional $20 \mathrm{~min}$. The samples were washed with WB1 and PNK buffer as before and 5' linker ligation was carried out overnight at $16^{\circ} \mathrm{C}$. The reaction was carried out in PNK buffer containing $1.25 \mu \mathrm{M}$ RA5 (N5) 5' linker, 40 U T4 RNA Ligase 1 (NEB) and $1 \mathrm{mM}$ ATP. The samples were washed with WB2 (50 mM Tris pH 7.8, $50 \mathrm{mM} \mathrm{NaCl}, 10 \mathrm{mM}$ imidazole, $0.1 \% \mathrm{NP}-40,5 \mathrm{mM} \beta$-mercaptoethanol) and the RNAprotein complexes were eluted by WB2 containing $150 \mathrm{mM}$ imidazole. The samples were subjected to TCA precipitation (as described in 4.2.7.5) and separated on a 4-12\% NuPAGE gel (Thermo Fischer Scientific) before transfer onto Hybond C membrane (Amersham). Radioactive signals were detected by autoradiography. Segments of the membrane carrying the radiolabelled RNA fragments were excised and RNAs were eluted from the membrane by incubating the membrane with WB2 supplemented with $1 \%$ SDS and $5 \mathrm{mM}$ EDTA overnight at $55^{\circ} \mathrm{C}$. RNA was extracted by addition of phenol:chloroform:isoamylalcohol (25:24:1) and $0.3 \mathrm{M}$ sodium acetate $\mathrm{pH}$ 5.2. The samples were centrifuged at $20000 \mathrm{rcf}$ for $5 \mathrm{~min}$ at RT. RNA was precipitated from the upper layer by addition of $20 \mu \mathrm{g}$ glycogen and 3 volumes of $100 \%$ ethanol. The samples were incubated at $-20^{\circ} \mathrm{C}$ overnight. Next, the samples were centrifuged at $20,000 \mathrm{rcf}$ for $20 \mathrm{~min}$ at $4^{\circ} \mathrm{C}$, and RNA pellet was washed with $70 \%$ ethanol and air dried. The pellet was resuspended in $1 \mu \mathrm{l}$ RTP primer (10 mM), $2 \mu \mathrm{l}$ dNTP mix ( $5 \mathrm{mM}$ ) and reverse transcription was carried out using Superscript III (Invitrogen) according to manufacturer's protocol. Next, PCR reactions were performed using $1 \mu \mathrm{l}$ of the above reaction along with $1 x \mathrm{LA}$ Tag buffer $+\mathrm{MgCl}_{2}, 0.2 \mu \mathrm{M} \mathrm{RPI}$ (Illumina forward index primer), $0.2 \mu \mathrm{M} R \mathrm{RP} 1$ (Illumina general reverse primer), $2.5 \mathrm{U}$ TakaRa La Taq (Clontech) and $1.25 \mathrm{mM}$ dNTPs. The PCR reaction conditions are summarized in 
Table 6. The DNA was precipitated using $0.3 \mathrm{M}$ sodium acetate $\mathrm{pH} 5.2$ and 3 volumes of $100 \%$ ethanol as described above. The pellet obtained was resuspended in $1 \times$ gel loading dye (Qiagen) and the PCR products were separated on a 3\% Metaphore agarose gel (Lonza) in 1x TBE. The PCR product was purified from the gel after excision using the MinElute gel extraction kit (Qiagen) according to manufacturer's protocol. The DNA concentration was determined using a Qubit Fluorometer (Thermo Fischer Scientific) and the cDNA library was subjected to Illumina deep sequencing. TestCRAC as well as full CRAC experiments and bioinformatics analyses have been performed in collaboration with Philipp Hackert and by Jens Kretschmer, respectively.

Table 6 PCR reaction conditions for CRAC

\begin{tabular}{l|l|l}
\hline \multicolumn{3}{l}{ PCR reaction components } \\
\cline { 1 - 2 } $95^{\circ} \mathrm{C}$ (denaturation) & $2 \mathrm{~min}$ & $\mathrm{x} 1$ \\
\hline $98^{\circ} \mathrm{C}$ (denaturation) & $30 \mathrm{sec}$ & \\
\cline { 1 - 2 } $60^{\circ} \mathrm{C}$ (annealing) & $40 \mathrm{sec}$ & \multirow{2}{*}{40} \\
\cline { 1 - 2 } $68^{\circ} \mathrm{C}$ (elongation) & $40 \mathrm{sec}$ & \\
\hline $72^{\circ} \mathrm{C}$ (elongation) & $5 \mathrm{~min}$ & $\times 1$ \\
\hline
\end{tabular}

\subsubsection{In vitro assays}

\subsubsection{Recombinant protein expression and purification}

BL21 (DE3) CodonPlus-RIL strain of E.coli was transformed (4.2.6.3) with the plasmid of interest for recombinant protein expression. Single colonies were then grown in LB media overnight at $37^{\circ} \mathrm{C}$ to obtain stationary phase cultures. The following day, the overnight cultures were used to inoculate 1 I LB cultures, such that the O.D600 was 0.1. Cultures were grown at $37^{\circ} \mathrm{C}$ until an O.D600 of 0.6 was reached, indicative of an exponential growth phase. For induction of recombinant protein expression, 1 mM IPTG was added and cultures were further grown for $16 \mathrm{~h}$ at $18^{\circ} \mathrm{C}$. The following day, cultures were harvested by centrifugation at $6,000 \mathrm{rcf}$ for $20 \mathrm{~min}$ at $4^{\circ} \mathrm{C}$. All subsequent purification steps were done on ice. The cell pellet was washed once with PBS before resuspension in $10 \mathrm{ml}$ lysis buffer (50 $\mathrm{mM}$ Tris- $\mathrm{HCl} \mathrm{pH}$ 7.0, $500 \mathrm{mM} \mathrm{NaCl}, 1 \mathrm{mM} \mathrm{MgCl}$, $10 \%$ glycerol, $1 \mathrm{mM}$ imidazole, $1 \mathrm{mM}$ PMSF). For cell lysis, cells were sonicated for $30 \mathrm{sec}, 4$ cycles with an amplitude of $45 \%$. Next, the lysate was cleared by centrifugation at 50,000 rcf for $20 \min$ at $4^{\circ} \mathrm{C}$. polyethyleneimine (PEI) was added to the clear lysate to a final concentration of $0.05 \%$ and further incubated for $15 \mathrm{~min}$ at $4^{\circ} \mathrm{C}$. The lysate was again centrifuged at 50,000 rcf for 30 
$\min$ at $4^{\circ} \mathrm{C}$ to precipitate nucleic acids. The lysate was then added to pre-equilibrated (with wash buffer I) cOmplete His-tag purification resin (Roche) and incubated for $2 \mathrm{~h}$. After incubation, the beads were washed with $10 \mathrm{ml}$ wash buffer I $(50 \mathrm{mM}$ Tris- $\mathrm{HCl} \mathrm{pH} \mathrm{7.0,500}$ $\mathrm{mM} \mathrm{NaCl}, 1 \mathrm{mM} \mathrm{MgCl} 2,10 \%$ glycerol, $30 \mathrm{mM}$ imidazole). This was followed by a high salt wash with $10 \mathrm{ml}$ wash buffer II (50 mM Tris- $\mathrm{HCl} \mathrm{pH} 7.0,1000 \mathrm{mM} \mathrm{NaCl}, 1 \mathrm{mM} \mathrm{MgCl} 2,10 \%$ glycerol, $30 \mathrm{mM}$ imidazole) followed by a final was with $10 \mathrm{ml}$ wash buffer I. Bound proteins were eluted with elution buffer ( $50 \mathrm{mM}$ Tris- $\mathrm{HCl} \mathrm{pH} 7.0,500 \mathrm{mM} \mathrm{NaCl}, 1 \mathrm{mM} \mathrm{MgCl}, 10 \%$ glycerol, $300 \mathrm{mM}$ imidazole). Elution fractions were spotted on to nitrocellulose membrane and stained with amido black. Protein-containing fractions were identified and pooled together, and glycerol was added to a final concentration of $20 \%$. The protein sample was transferred to SpectraPore dialysis membrane with a cut off of $15 \mathrm{kDa}$. Dialysis was carried out overnight at $4^{\circ} \mathrm{C}$ in dialysis buffer (50 mM Tris- $\mathrm{HCl} \mathrm{pH} \mathrm{7.0,150} \mathrm{mM} \mathrm{NaCl}, 1 \mathrm{mM} \mathrm{MgCl}$, $20 \%$ glycerol). The following day, the dialysed protein sample was concentrated to an appropriate volume using a SpinX UF concentrator (Corning) with a molecular cut off of 30 $\mathrm{kDa}$. Protein samples were aliquoted and stored at $-80^{\circ} \mathrm{C}$.

\subsubsection{Steady-state ATPase assay}

Hydrolysis of ATP was monitored with an NADH-coupled reaction (Kiianitsa et al., 2003). Reactions were set up containing $1.5 \mu \mathrm{M}$ protein sample, $45 \mathrm{mM}$ Tris- $\mathrm{HCl} \mathrm{pH} 7.4,2 \mathrm{mM}$ $\mathrm{MgCl}_{2}, 25 \mathrm{mM} \mathrm{NaCl}, 450 \mu \mathrm{M}$ NADH ,1.5 mM phosphoenolpyruvate (PEP, Sigma-Aldrich), $4 \mathrm{mM} \mathrm{ATP}$ and $20 \mathrm{U} / \mathrm{ml}$ pyruvate kinase/lactate dehydrogenase (Sigma-Aldrich) supplemented with $1.5 \mu \mathrm{M}$ RNA. Hydrolysis of ATP leads to a rise in ADP levels which when converted back into ATP results in the conversion of phoenolpyruvate to pyruvate by the action of pyruvate kinase. In the next step, the conversion of pyruvate to lactate by lactate dehydrogenase is concomitantly linked to the oxidation of NADH to NAD+. The decrease in absorbance of NADH at $340 \mathrm{~nm}$ was measured using a BioTEK Synergy HT microplate spectrophotometer. The rate of ATP hydrolysis was calculated using the following equation:

$$
\text { ATPase rate }\left[\frac{\mathrm{ATP}}{\min }\right]=\frac{\frac{\mathrm{dA} 340}{\mathrm{dt}}\left[\frac{\mathrm{OD}}{\mathrm{min}}\right]}{\text { Kpath } * \text { moles ATP }}
$$

Wherein, $\mathrm{K}_{\text {path }}$ is the molar absorption co-efficient for a defined optical path length which is defined as reaction volume $(150 \mu \mathrm{l} /$ well $)$ and background NADH decomposition.

\subsubsection{SDS-PAGE and western blotting}

Protein samples were analysed by SDS-polyacrylamide gel electrophoresis (SDS-PAGE) following Laemmli's instructions (Laemmli, 1970). Samples were mixed with 4x SDS loading 
dye (8\% SDS, $200 \mathrm{mM}$ Tris-HCl pH 6.8, 4\% $\beta$-mercaptoethanol, $50 \mathrm{mM}$ EDTA, 0.08\% bromophenol blue and $40 \%$ glycerol) and heated at $95^{\circ} \mathrm{C}$ for $10 \mathrm{~min}$. SDS-PAGE was carried out for $1.5 \mathrm{~h}$ with $25 \mathrm{~mA} / \mathrm{gel}$ in a discontinuous system consisting of different stacking (125 mM Tris- $\mathrm{HCl} \mathrm{pH}$ 6.8, $3.5 \mathrm{mM}$ SDS) and resolving (375 mM Tris- $\mathrm{HCl} \mathrm{pH}$ 8.8, $3.5 \mathrm{mM}$ SDS) buffers in a Bio-rad system. The gels were either stained with Coomassie solution (0.1\% Coomassie R-250, 10\% acetic acid, 40\% methanol) or transferred onto nitrocellulose membrane (GE-healthcare) via wet blotting. Proteins were transferred for $1 \mathrm{~h}$ at $100 \mathrm{~V}$ using a Bio-rad transfer set up in 1X western blotting buffer (25 mM Tris, $192 \mathrm{mM}$ glycine, $0.05 \%$ SDS, $20 \%$ methanol). After transfer, the membrane was incubated for $1 \mathrm{~h}$ with blocking solution containing $5 \%$ milk in TBS $(25 \mathrm{mM}$ Tris- $\mathrm{HCl} \mathrm{pH} 7.4,137 \mathrm{mM} \mathrm{NaCl}, 2.7 \mathrm{mM} \mathrm{KCl})$ with $0.1 \%$ Tween-20 (TBST-T) in order to avoid unspecific antibody binding to the membrane. After blocking, membrane was incubated with primary antibodies diluted in blocking solution and incubated overnight at $4^{\circ} \mathrm{C}$. The membrane was washed three times, 10 min each time with TSBT-T followed by incubation for $1 \mathrm{~h}$ with secondary antibodies diluted in blocking solution. Membranes were again washed as described above and the proteins were detected using Odyssey CLx imager (Li-COR Biosciences). 


\subsection{Results}

\subsubsection{DDX55 is an ATPase and a mutation in its conserved GKT motif inhibits its}

\section{ATP hydrolysis capacity}

In yeast, the DEAD-box helicase Spb4 was recently shown to be involved in ribosome biogenesis (Brüning et al., 2018). Based on its homology to DDX55, we speculated DDX55 to be a putative RNA helicase that may be involved in ribosome biogenesis in humans. Many RNA helicases hydrolyse nucleotide triphosphates (NTPs) in an RNA-dependent manner (Jarmoskaite and Russell, 2014; Linder and Jankowsky, 2011). Hence, we set out to explore if DDX55 is an ATPase. We, therefore, expressed recombinant, His-tagged DDX55 in E. coli cells and purified protein for performing in vitro ATP hydrolysis assays. In many RNA helicases, a characteristic glycine-lysine-threonine (GKT) sequence in the walker A motif is responsible for RNA binding and ATP hydrolysis. To confirm the specificity of any ATPase activity observed, a mutant form of DDX55 carrying a threonine to alanine substitution in the GKT motif at amino acid position 60 was also expressed and purified (Figure 4.1A).
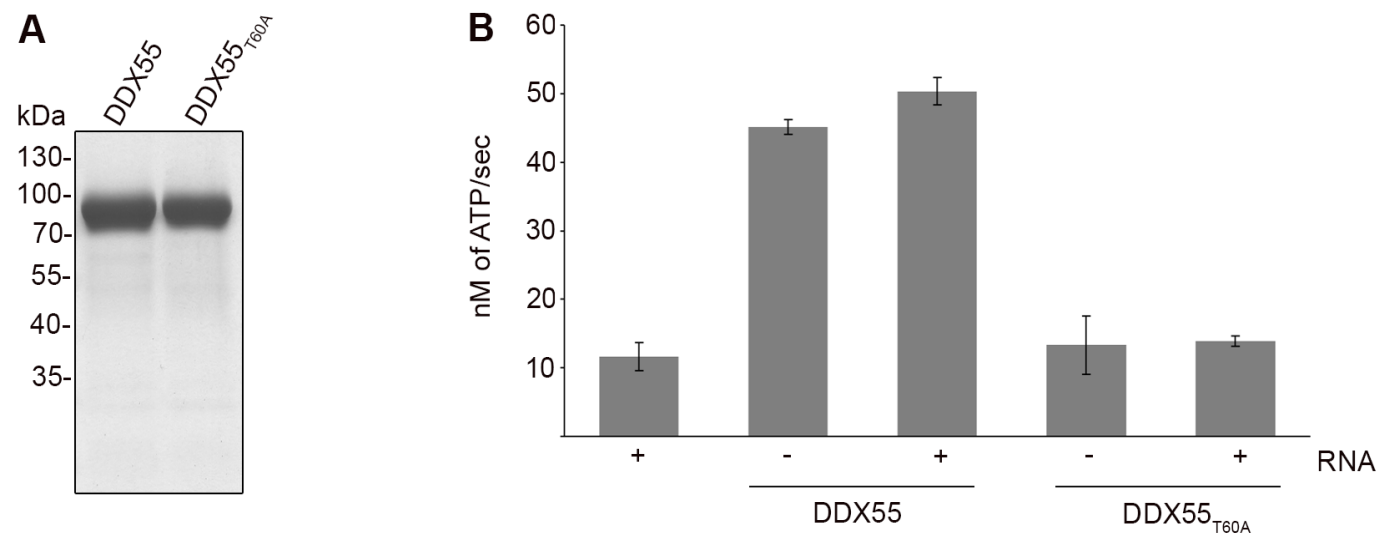

Figure 4.1. DDX55 is an ATPase. (A) ZZ-DDX55-His ${ }_{7}$ and ZZ-DDX55 ${ }_{\text {T60A }}-\mathrm{His}_{7}$ were expressed in E. coli and purified by affinity chromatography. The elution fractions containing protein were dialysed and concentrated. Proteins were separated by SDS-PAGE and visualized by Coomaissie staining. (B) Rate of ATP hydrolysed by DDX55 and DDX55 60 A was determined by monitoring the absorbance at $340 \mathrm{~nm}$. Reactions were carried out in the presence (+) or absence of RNA (-). Experiments were performed in triplicates and error bars represent mean \pm standard deviation.

ATP hydrolysis was then monitored with an NADH-coupled reaction and was performed with DDX55 or DDX55T60A in the presence or absence of RNA. In comparison to the control sample containing no helicase, DDX55 exhibited strong ATP hydrolysis activity even in the absence of an RNA substrate and the addition of RNA resulted in mild stimulation of the ATPase activity. In contrast, the mutant variant of DDX55 only weakly hydrolysed ATP in 
the absence of RNA and the addition of RNA into the reaction mixture did not stimulate ATP hydrolysis. This implies that DDX55 is an active ATPase and the T to A substitution within the conserved GKT motif is sufficient to abolish the ATPase activity of DDX55 (Figure 4.1B).

\subsubsection{The human helicase DDX55 is required for ribosome biogenesis}

\subsubsection{DDX55 is localised in the nucleoplasm}

Having established that DDX55 is an active ATPase, the next objective was to analyse DDX55 in vivo and investigate whether it has a role in ribosome biogenesis. First, we determined which sub-cellular compartment DDX55 is present in. Due to lack of a functional DDX55-specific antibody that could be used for immunofluorescence, an inducible HEK293 stable cell line expressing DDX55-Flag was generated and the localisation of Flag-tagged DDX55 was determined using an antibody against the Flag-tag. The SSU biogenesis factor, UTP14A and DAPI were used for visualisation of nucleoli and nuclear material, respectively. The localisation of DDX55 was observed to be predominantly nucleoplasmic and the helicase seemed to be largely excluded from the nucleoli (Figure 4.2), suggesting that DDX55 could be involved in late stages of ribosome biogenesis.

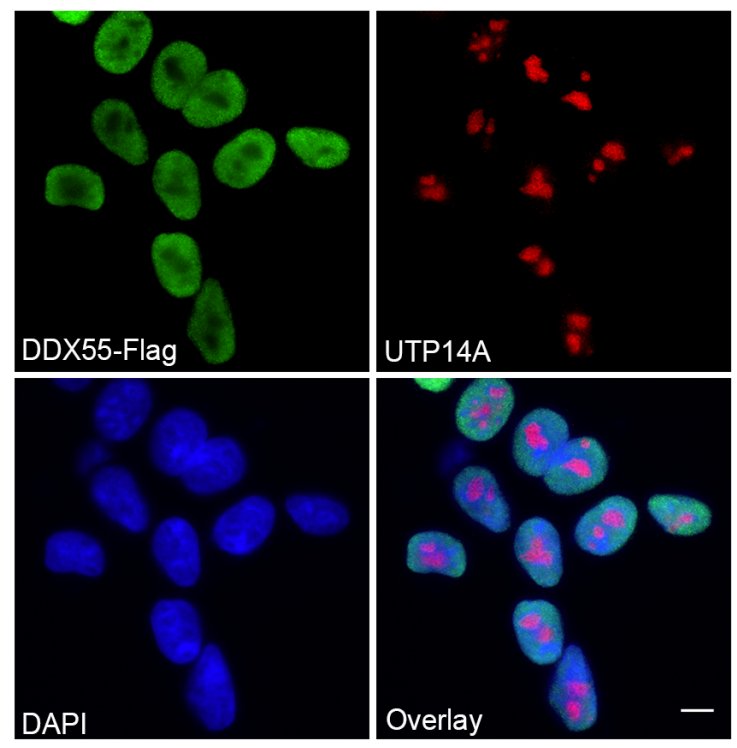

Figure 4.2. DDX55 predominantly exhibits a nucleoplasmic localisation. Expression of DDX55-Flag was induced in a HEK293 stable cell line for $24 \mathrm{~h}$ with tetracycline and cells were fixed with $4 \%$ paraformaldehyde for immunofluorescence microscopy. The localisation of DDX55 was determined using an antibody against the Flag-tag (green). UTP14A, an SSU biogenesis protein, served as nucleolar marker (red) and the nuclear material was visualized by staining with DAPI (blue). An overlay of images in presented for the purpose of co-localisation analysis. Scale bar represents $10 \mu \mathrm{m}$. 


\subsubsection{Depletion of DDX55 in vivo impairs ribosome biogenesis}

To analyse the potential role of DDX55 in ribosome biogenesis, RNA interference (RNAi) was established using different siRNAs. HeLa cells were either left untreated or were treated with a non-target siRNA (siNT) or siRNAs targeting DDX55 (siRNA1-4). Cells were harvested $72 \mathrm{~h}$ after transfection and levels of DDX55 protein were determined using western blotting. In contrast to the cells treated with the non-target siRNA, there was a marked reduction in the DDX55 level upon treatment with siRNAs 1,2 and 4 against DDX55. Furthermore, siRNA_2 and siRNA_4 were equally efficient in depleting DDX55 and were used for further experiments (Figure 4.3). Tubulin was used as a loading control.

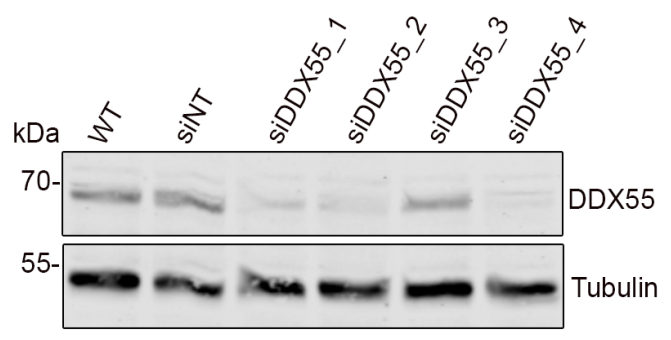

Figure 4.3. siRNA-mediated depletion of DDX55. HeLa cells were transfected with a non-target siRNA (siNT), siRNAs against DDX55 (1-4) or left untransfected (WT) for $72 \mathrm{~h}$. Cells were harvested and proteins were separated by SDS-PAGE and analysed by western blotting with antibodies against DDX55 and tubulin.

Upon RNAi-mediated depletion of DDX55, the levels of newly synthesized (pre-) rRNAs were analysed by pulse-chase labelling. HeLa cells were transfected with non-target siRNA or siRNA targeting DDX55 (siRNA_2 or siRNA_4). After $96 \mathrm{~h}$, cells were first grown in the presence of phosphate-free DMEM followed by growth in the presence of DMEM containing $\left[{ }^{32} \mathrm{P}\right]$-labelled orthophosphate which is incorporated into the nascent RNAs. After $1 \mathrm{~h}$, labelled media were exchanged for DMEM and cells were harvested at specific time points. Extracted total RNA was separated on an agarose gel and transferred to a nylon membrane. The newly-synthesized, labelled rRNAs were detected by a phosphoimager. Steady-state levels of mature $18 \mathrm{~S}$ and $28 \mathrm{~S}$ rRNAs were visualized by methylene blue staining. Actin mRNA, which served as a loading control, was detected via northern blotting by using a radiolabelled probe. Depletion of DDX55 led to a marked reduction in the levels of newly synthesized $28 \mathrm{~S}$ and $18 \mathrm{~S}$ rRNAs when compared to the levels of actin mRNA. The steady state levels of mature $18 \mathrm{~S}$ and $28 \mathrm{~S}$ were also decreased as visualized by methylene blue staining (Figure 4.4). This indicates that loss of DDX55 results in impaired ribosome biogenesis in the cell. 


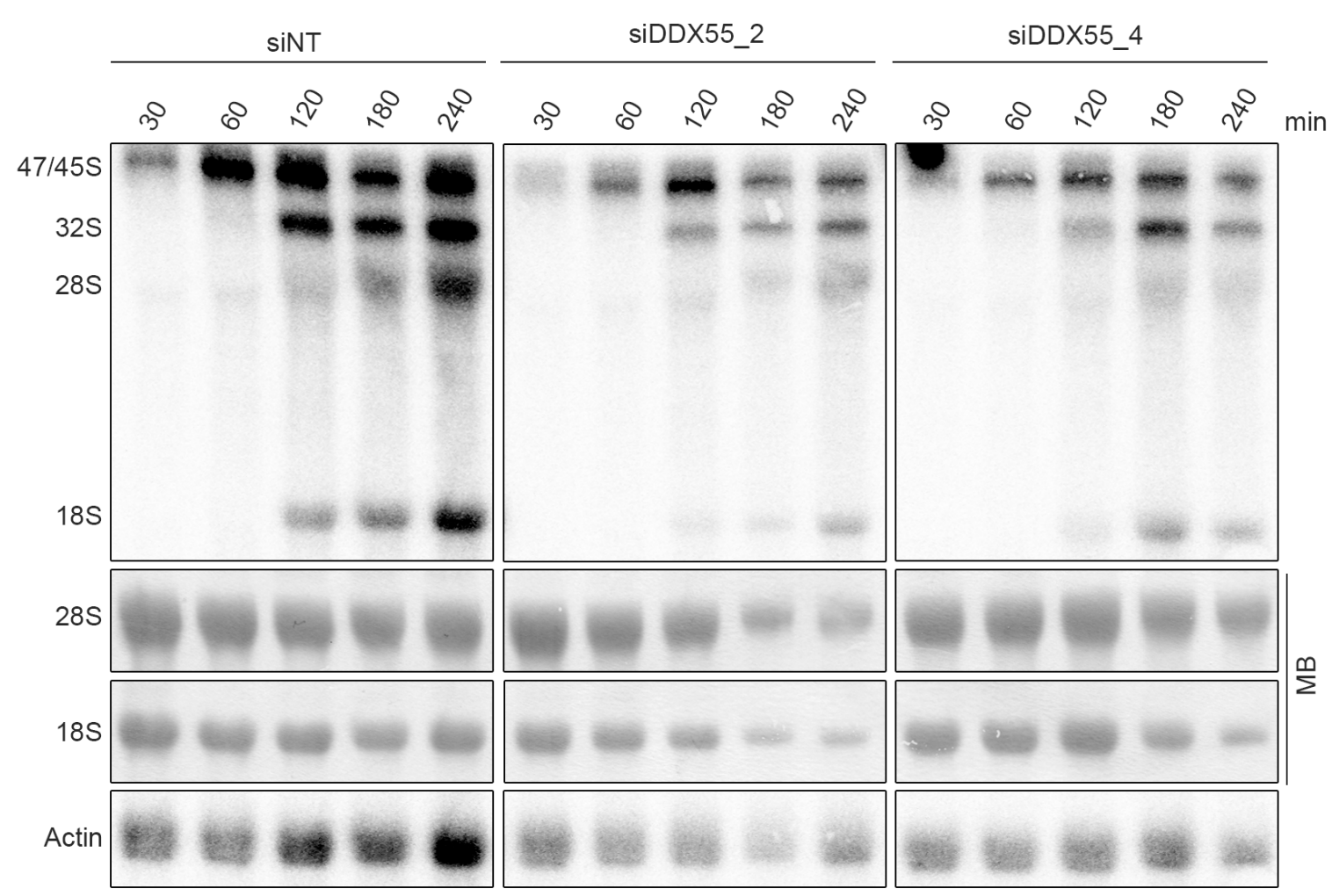

Figure 4.4. siRNA-mediated depletion of DDX55 impairs production of rRNAs. HeLa cells were transfected with a non-target siRNA (siNT) or siRNAs targeting DDX55 (siDDX55_2 and DDX55_4). After $96 \mathrm{~h}$, cells were grown in phosphate-free media for $1 \mathrm{~h}$, followed by growth in media containing $\left.{ }^{32} \mathrm{P}\right]-$ labelled orthophosphate for labelling nascent RNAs. Labelled media was exchanged with complete DMEM and cells were harvested at specific time points. Total RNA was extracted and separated on $1.2 \%$ agarose gel and transferred to a nylon membrane. Nascent, labelled RNAs were detected using a phosphoimager. Steady-state levels of $28 \mathrm{~S}$ and $18 \mathrm{~S}$ were visualized by methylene blue (MB) staining. The actin mRNA, which was detected with a specific radiolabelled probe, served a loading control.

\subsubsection{DDX55 crosslinks to helices 62 and 63 of the 28S rRNA sequence in vivo}

In order to understand the precise role of RNA helicases in ribosome biogenesis, it is imperative to identify their binding site(s) on target RNAs in vivo. Crosslinking and analysis of $\underline{\text { cDNA }}$ (CRAC) was, therefore, employed to reveal the in vivo crosslinking site(s) of DDX55 (Bohnsack et al., 2012; Haag et al., 2017). HEK293 cells expressing either Flagtagged DDX55 or the Flag-tag alone were crosslinked in vivo using UV light at $254 \mathrm{~nm}$. After crosslinking, a two-step purification approach was utilized in order to ensure recovery of only RNA sequences, which were specifically bound by Flag-tagged DDX55 or by the Flagtag. Purification was carried out first under native conditions using an immobilised anti-Flag antibody followed by a Ni-NTA-based purification under denaturing conditions. Crosslinked RNA was partially digested in order to trim away RNA sequences not directly in contact with Flag-tagged DDX55 or the Flag-tag alone and the trimmed RNA fragments were ligated to linkers and labelled with $\left[{ }^{32} \mathrm{P}\right]$. After elution, the covalently crosslinked RNA-protein 
complexes were separated by denaturing PAGE, transferred to a nitrocellulose membrane and detected by autoradiography (Figure 4.5). RNA was extracted from the regions corresponding to the molecular weight of DDX55, reverse transcribed and PCR amplified to generate a cDNA library, which was utilized for next generation sequencing.

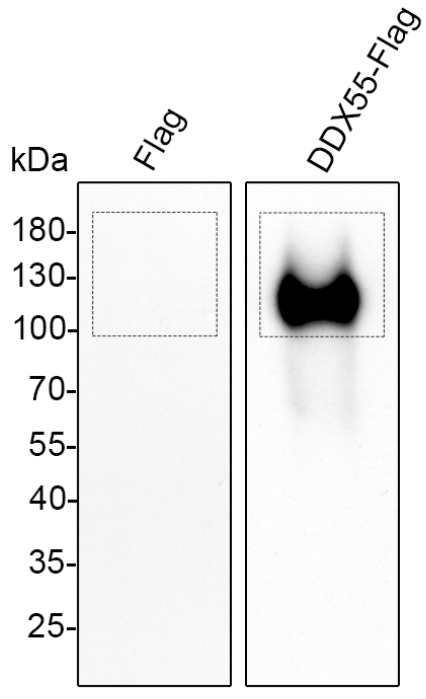

Figure 4.5. DDX55 crosslinks to RNA in vivo. HEK293 cells expressing either the Flag-tag control or DDX55-Flag were crosslinked in vivo using UV light at $254 \mathrm{~nm}$. RNA-protein complexes were purified under both native and denaturing conditions. After RNase treatment, RNA sequences specifically associated with the protein were ligated to sequencing adaptors and labelled with [32P]. The RNAprotein complexes were eluted and separated by denaturing PAGE. After transfer to a nylon membrane, the labelled RNAs were detected by autoradiography. The regions along the dotted line were excised, RNA extracted and a cDNA library was generated for next generation sequencing.

The reads obtained after Illumina sequencing were bioinformatically mapped to the human genome. There was a prominent enrichment of reads corresponding to ribosomal RNA in the Flag-tagged DDX55 (31\%) in comparison to the Flag-tag only (14\%) (Figure 4.6), which is consistent with its role in ribosome biogenesis. An increase in the number of reads mapping to tRNA genes was also observed in case of DDX55-Flag (22\%) compared to Flag-tag control $(3 \%)$. However, as no specific tRNA was enriched this likely represents background. As tRNAs are abundant in nature, they are often non-specifically co-purified with RNA-binding protein and have been previously observed to be non-specifically enriched in other CRAC samples. 


\section{Flag}

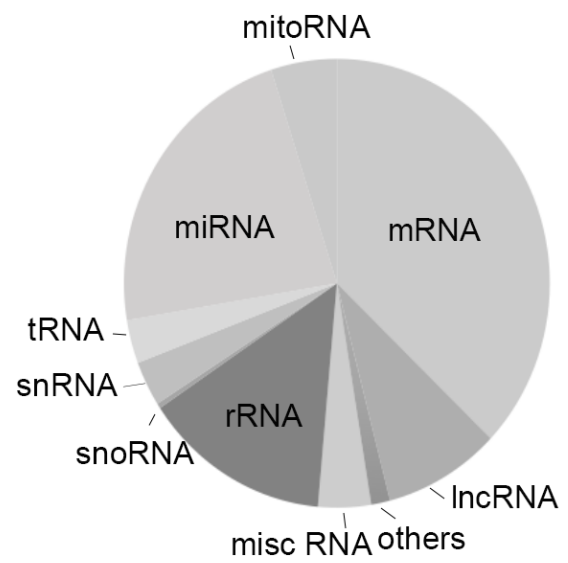

DDX55-Flag

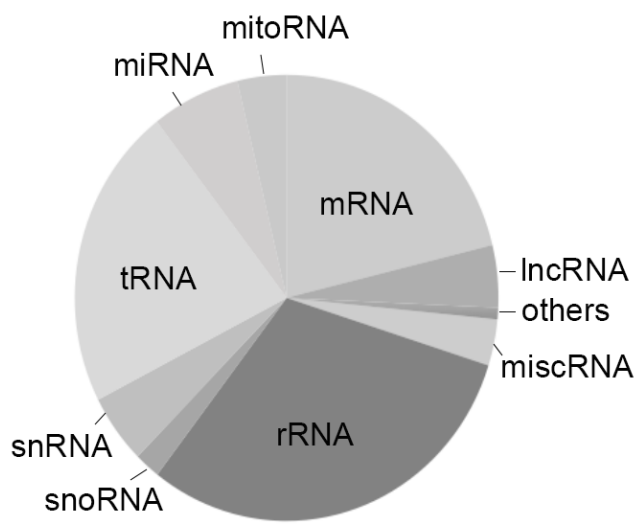

Figure 4.6. Flag-tagged DDX55 enriches ribosomal RNAs. In vivo crosslinked protein-RNA complexes were tandem affinity purified under native and denaturing conditions and crosslinked RNAs were partially digested, labelled with $\left[{ }^{32} \mathrm{P}\right]$ and ligated to adaptors for generation of a cDNA library. The library was then subjected to Illumina deep sequencing and the reads obtained were mapped to the human genome. Pie charts represent the relative distribution of sequence reads mapping to different classes of RNA. Abbreviations: mRNA, messenger RNA; IncRNA, long non-coding RNA; snoRNA, small nucleolar RNA; snRNA, small nuclear RNA; tRNA, transfer RNA; mitoRNA, mitochondrial RNA; miRNA, microRNA; rRNA, ribosomal RNA; miscRNA, miscellaneous RNA.

As an increase in the number of reads mapping to the rDNA sequence was observed for DDX55, we next wanted to determine the specific crosslinking site within the rRNA. The number of reads at each nucleotide was normalized according to the total number of mapped reads per million and mapped at single nucleotide level on to the rDNA locus encoding the $47 \mathrm{~S}$ pre-rRNA transcript. Increased accumulation of reads in specific peaks represents a specific crosslinking site for the analysed protein. In comparison to the Flagtag control, a specific peak was obtained on the 28S rRNA sequence for DDX55, consistent with its role in LSU biogenesis (Figure 4.7). 


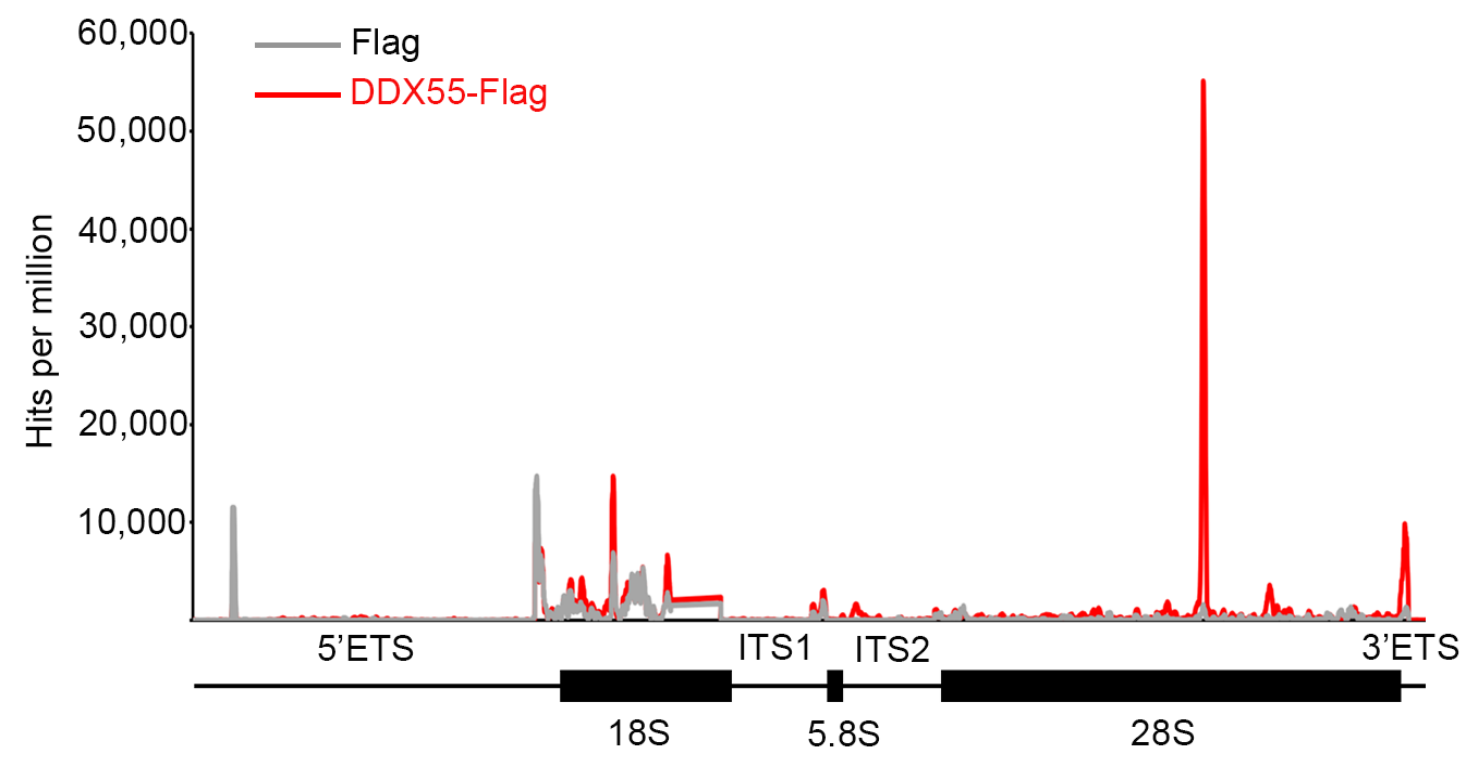

Figure 4.7. DDX55 crosslinks to a specific site within the 28S rRNA sequence. The sequence mapping to rDNA locus encoding the $47 \mathrm{~S}$ ribosomal RNA transcript are shown. The reads obtained for Flag and DDX55-Flag are depicted in grey and red respectively. A schematic representation of the $47 \mathrm{~S}$ rRNA is depicted below and wherein the mature rRNA sequences are depicted as rectangles and those of the spacer regions are depicted as lines.

In order to better understand the functional relevance of the distinct crosslinking site obtained, the DDX55-Flag CRAC data were mapped onto the secondary structure of mature LSU RNAs (Petrov et al., 2014). The number of sequence reads mapped to each nucleotide of the LSU RNAs is depicted as a colour gradient, with red representing the highest number of reads normalised to $100 \%$. This revealed that DDX55 preferentially crosslinks to helices 62 and 63 of domain IV within 28S rRNA sequence, in the vicinity of the eukaryotic expansion segment 27 (ES27) (Figure 4.8). Interestingly, in yeast, Spb4 binds to helices 62 and 63 of the 25S rRNA sequence suggesting that the binding site of these helicases is evolutionarily conserved in eukaryotes (Brüning et al., 2018).

Additionally, the DDX55-CRAC data were mapped onto the available tertiary structure of the mature large ribosomal subunit (PDB 4V6X, Anger et al., 2013) in order to visualise the binding site of DDX55 relative to other ribosomal features (Figure 4.9). 


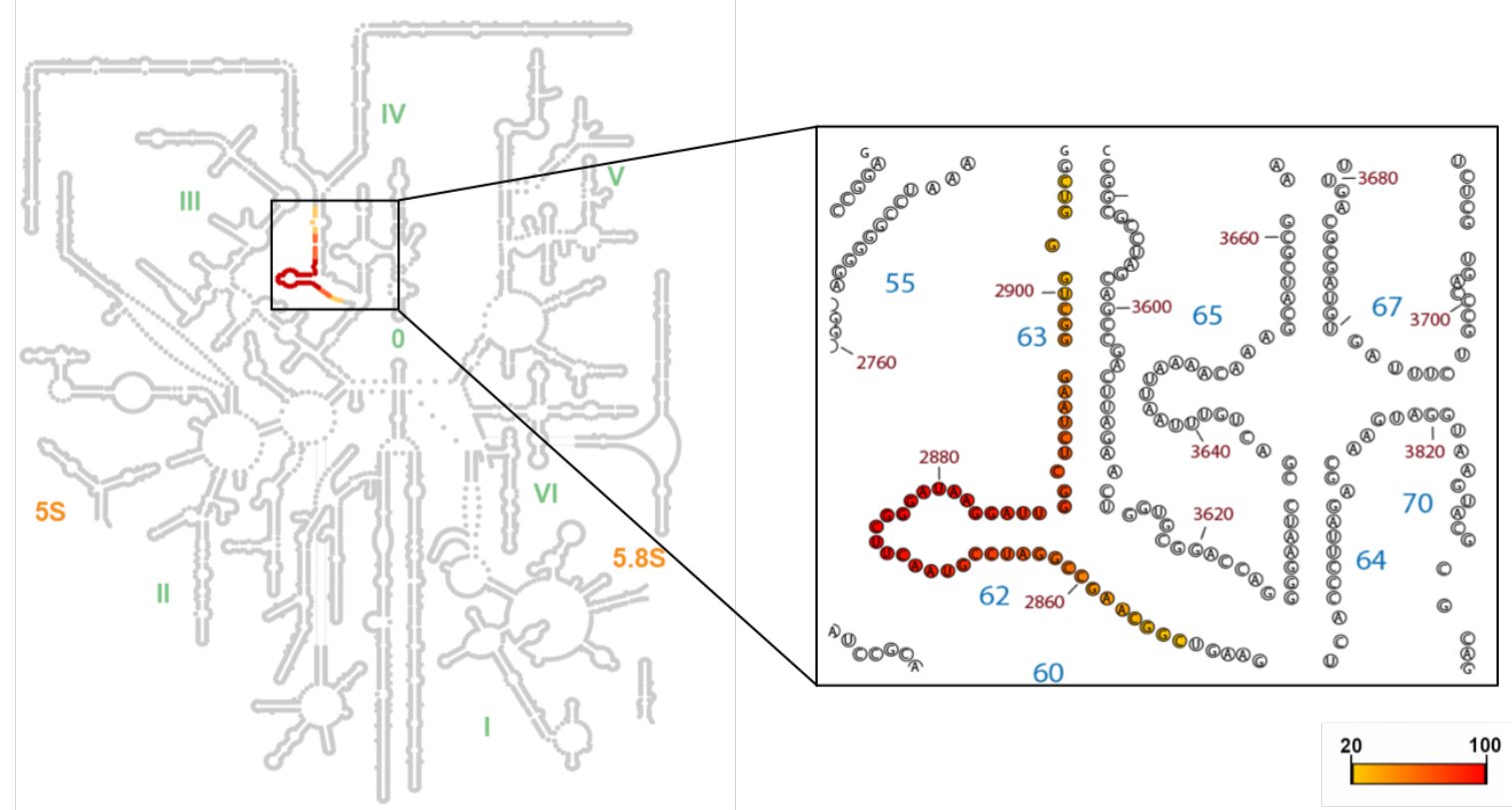

Figure 4.8. DDX55 crosslinks to helices 62 and 63 of the $28 \mathrm{~S}$ rRNA. The sequence reads obtained for DDX55-Flag CRAC data were mapped on the secondary structure of LSU RNAs. 5S and 5.8S are labelled in brown. Various domains of 28S rRNA is depicted in green. Crosslinking sites are represented as a gradient ranging from yellow (lowest value, $20 \%$ above threshold) to red (highest value).

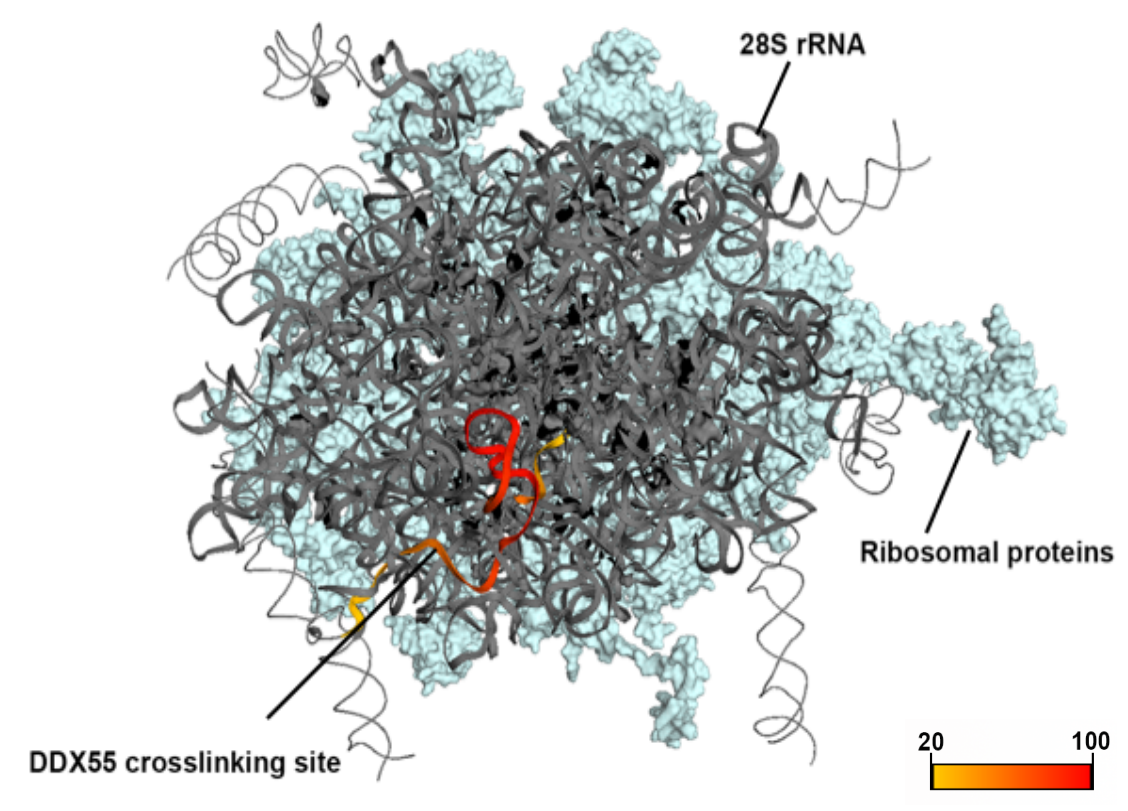

Figure 4.9. DDX55 crosslinks to helices 62 and 63 in domain IV of the 28S rRNA. The sequence reads obtained for DDX55-Flag CRAC data were mapped on the 3D structure of the 28S rRNA. Ribosomal proteins are depicted in blue. Crosslinking sites are represented as a gradient ranging from yellow (lowest value, $20 \%$ above threshold) to red (highest value). 


\subsubsection{C-terminal tail of DDX55 is required for its recruitment to pre-ribosomal subunits}

Having established that DDX55 is an active ATPase that associates with pre-ribosomal complexes and is required for their maturation, we further explored the mechanism of DDX55 binding to RNA and its recruitment to ribosomal subunits. Interestingly, detailed sequence characterization revealed clusters of basic amino acid residues localised within the C-terminal tail region of DDX55 (Figure 4.10).
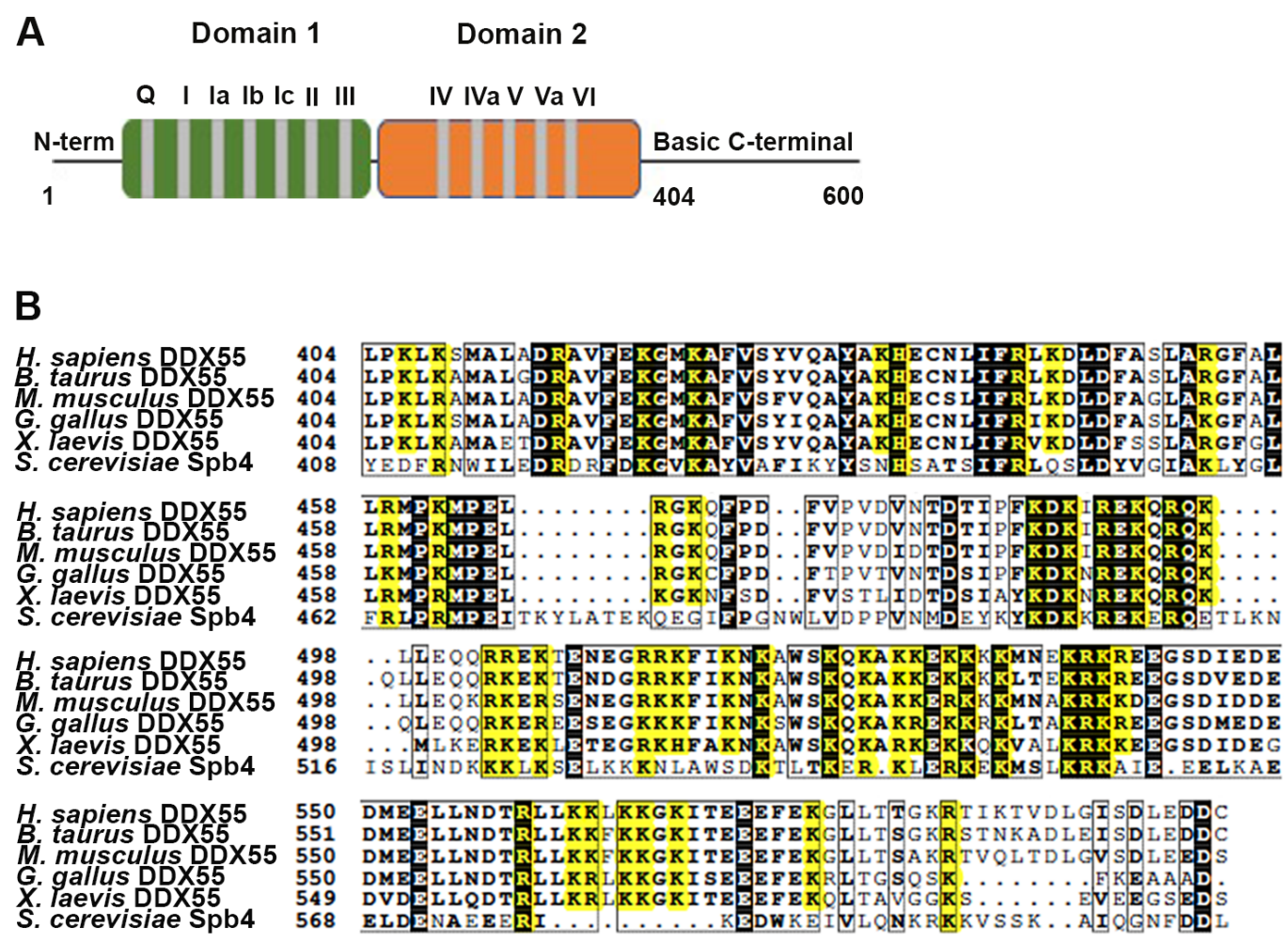

Figure 4.10. C-terminal extension of DDX55 contains conserved basic amino acid residues (A) Schematic representation of primary sequence of DDX55 depicting the two conserved RecA-like domains, domain 1 (green) and domain 2 (orange). Each domain consists of several conserved motifs coloured in grey. The $\mathrm{N}$ - and C-terminal extensions are denoted. (B) Sequence alignment of amino acid residues from the C-terminal tail region (residues 404-600) is represented from S. cerevisiae Spb4 (Uniprot: P25808); H. sapiens DDX55 (Uniprot: Q8NHQ9); B. taurus DDX55 (Uniprot: Q2NL08); G. gallus DDX55 (Uniprot: Q5ZLN8); M. musculus DDX55 (Uniprot: Q6ZPL9) and X. laevis DDX55 (Uniprot: Q6AZV7). Conserved amino acid are highlighted in black and basic amino acid residues are marked in yellow.

To enable potential functions of the C-terminal tail of DDX55 to be investigated, HEK293 stable cell lines expressing Flag-tagged full-length DDX55 and a C-terminally truncated version of DDX55, consisting of amino acids residues 1 to 403 were generated (DDX55 $\triangle$ Cterm). Previous studies have suggested that basic C-terminal domains can contribute to the recruitment of helicases to their site of action by mediating interactions with RNA. For 
example, the predominantly basic C-terminal extension of DEAD-box proteins Mss116 in yeast and CYT-19 in Neurospora crassa are involved in facilitating interaction of the helicase with its RNA or RNP targets (Mohr et al., 2008). In vivo crosslinking experiments were therefore carried out with cell lines expressing full-length or truncated DDX55 wherein the protein of interest was UV-crosslinked to associated RNAs. The RNAs after crosslinking were radiolabelled with $\left[{ }^{32} \mathrm{P}\right]$, then protein-RNA complexes were separated by denaturing PAGE, transferred to a nitrocellulose membrane and detected by autoradiography. The elution fractions were also analysed by SDS-PAGE followed by western blotting with an anti-Flag antibody to confirm that similar amounts of the full-length and truncated DDX55 proteins were loaded (Figure 4.11A). In the case of Flag-tagged full-length DDX55, a strong signal migrating at the molecular weight of DDX55 was obtained, confirming the direct interaction of DDX55 with RNA in vivo. However, a significantly weaker signal was observed in the case of truncated DDX55, indicating a loss in RNA binding upon truncation of the Cterminal basic tail (Figure 4.11B).
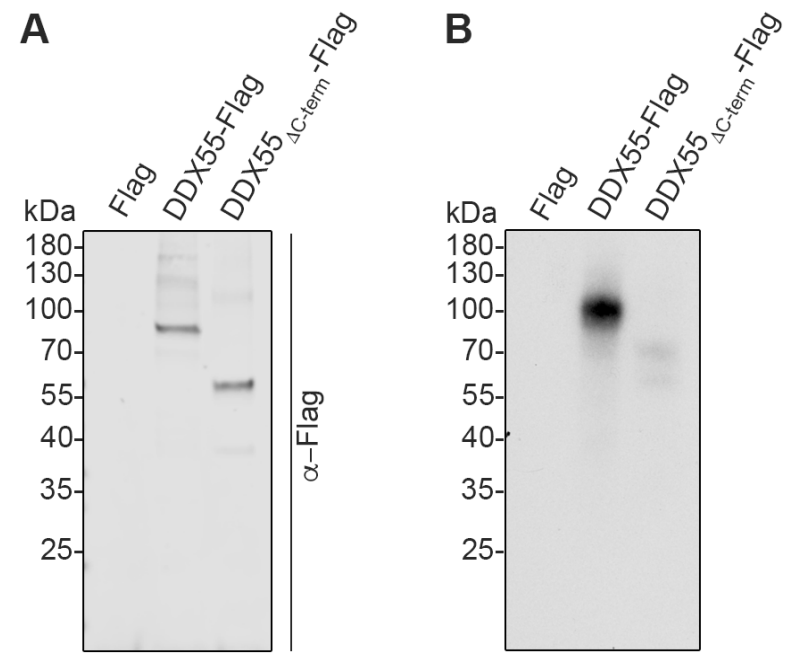

Figure 4.11. Deletion of the C-terminal tail of DDX55 leads to decreased RNA binding. HEK293 stable cell lines expressing DDX55-Flag or DDX55 $\triangle$ C-term -Flag were crosslinked in vivo using UV light at $254 \mathrm{~nm}$. RNA-protein complexes were purified first under native and then under denaturing conditions. Crosslinked RNA was partially digested and $\left[{ }^{32} \mathrm{P}\right]$ labelled. Complexes were separated by denaturing PAGE and transferred onto nitrocellulose membranes. (A) The amount of Flag-tagged DDX55 and DDX55 $\triangle \mathrm{C}$-term in the elution fractions was detected by western blotting with antibody against the Flag-tag. (B) Radiolabelled RNAs were detected by autoradiography.

Immunofluorescence microscopy was then performed in HEK293 stable cell lines expressing Flag-tagged DDX55 and the truncated Flag-tagged DDX55 $5_{\Delta \mathrm{C} \text {-term }}$ using an antiFlag antibody in order to examine the effect of deleting the tail on the sub-cellular localisation of the helicase. Full-length DDX55 was predominantly present in the nuclear 
compartment, co-localizing with the nuclear stain, DAPI, as previously observed (Figure 4.12A). In contrast, DDX55 lacking the C-terminal tail was largely excluded from the nuclear compartment and a dispersed signal was detected in the cytosol (Figure 4.12B).

A
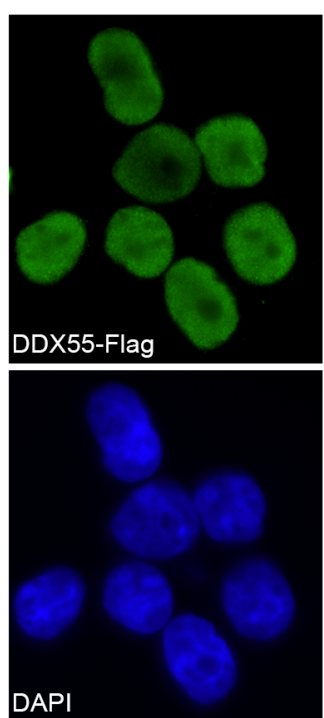
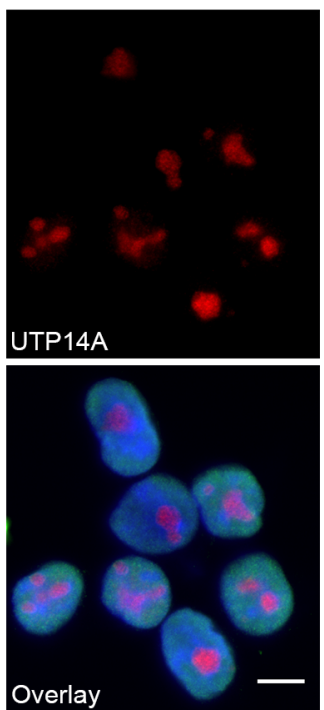

B
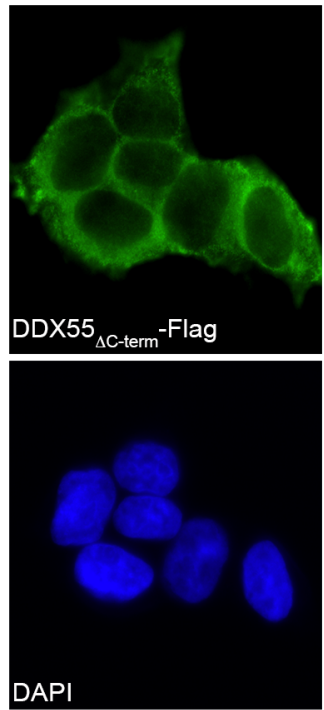
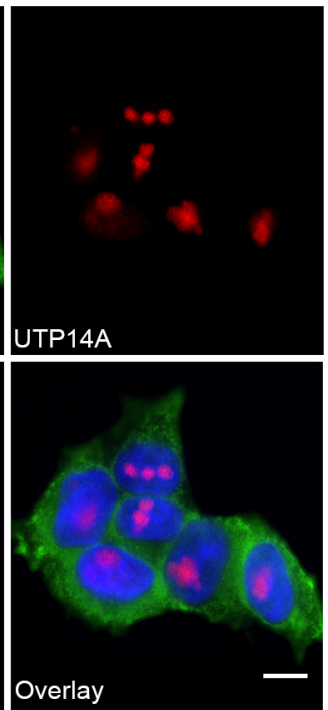

Figure 4.12. C-terminal truncation of DDX55 causes its mis-localisation to the cytoplasm. (A-B). Stable cell lines for the expression of DDX55-Flag (A) or DDX55 4 C-term - Flag (B) were induced with tetracycline for $24 \mathrm{~h}$ before fixing with $4 \%$ paraformaldehyde. The localisation of DDX55-Flag or DDX55 $\triangle \mathrm{C}$ term-Flag were determined by immunofluorescence with an anti-Flag antibody (green). Immunofluorescence against UTP14A (red) served as a nucleolar marker. The nuclear material was visualized with DAPI (blue). An overlay of images is presented and the scale bar corresponds to $10 \mu \mathrm{m}$.

As the C-terminal tail of DDX55 was observed to be important for RNA binding and nucleoplasmic localisation of the helicase in vivo, its potential role in recruitment of the helicase to the pre-ribosomal subunits was next analysed. Whole cell lysates from HEK293 stable cells lines expressing full length Flag-tagged DDX55 or the truncated DDX55 ${ }_{\Delta \mathrm{C} \text {-term- }}$ Flag were separated on sucrose density gradients. The absorbance of each fraction at 260 $\mathrm{nm}$ was measured to determine the RNA content and, thereby enable the fractions containing the large and small ribosomal subunits, and $80 \mathrm{~S}$ monosomes to be identified. SDS-PAGE and western blotting with anti-Flag antibody was then used to analyse the distribution of the Flag-tagged proteins within the gradient. Full-length DDX55 was enriched in fractions containing 60S subunits, implying that it predominantly associates with pre-60S particles in the nucleus. As expected, there was a dramatic reduction in the amount of truncated DDX55 present in these fractions, demonstrating the requirement for the basic $\mathrm{C}$ terminal tail of DDX55 for recruitment of the helicase to the right sub-cellular compartment and hence, its site of action in pre-60S complexes (Figure 4.13). 


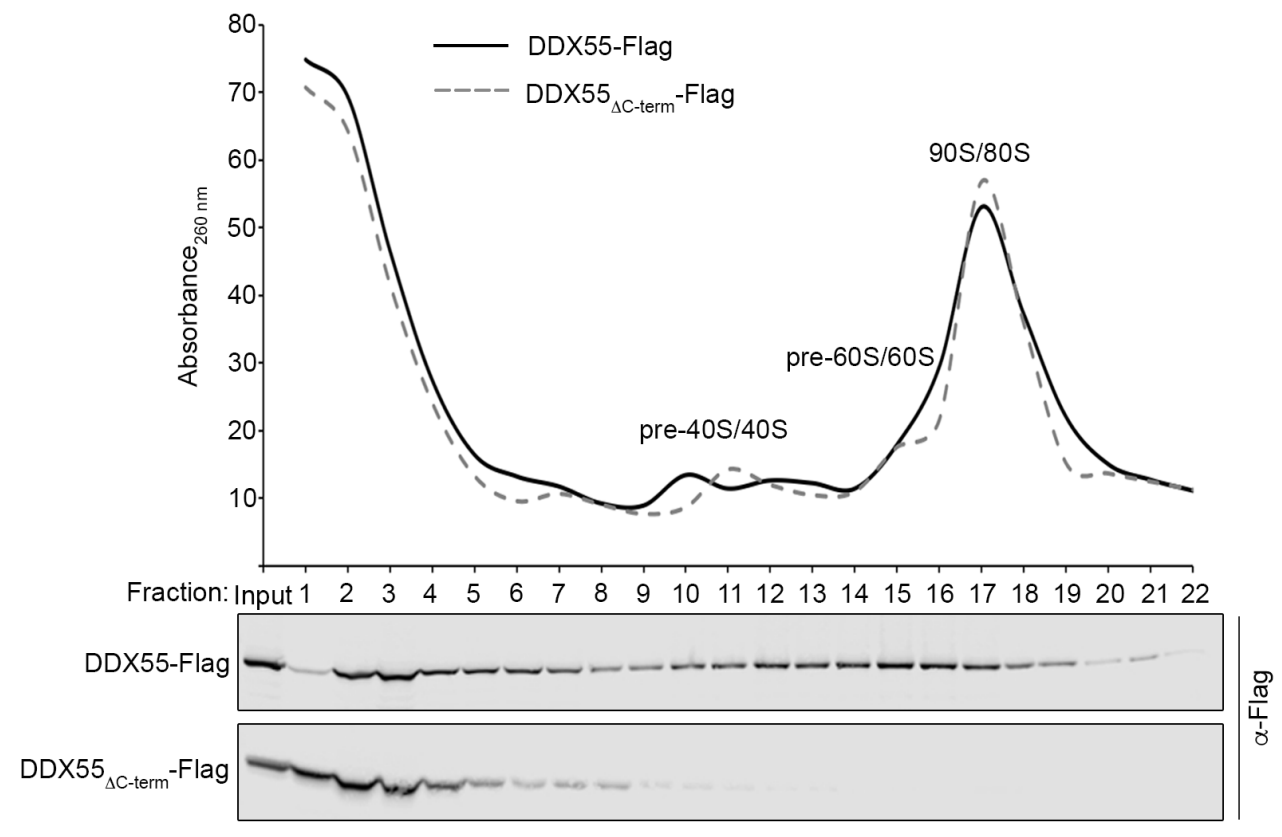

Figure 4.13. Deletion of the C-terminal basic tail of DDX55 results in reduced association with preribosomal subunits. Flag-tagged DDX55 and DDX55 ${ }_{\triangle C \text {-term }}$-Flag were expressed from HEK293 cells by addition of tetracycline. After $24 \mathrm{~h}$, cells were harvested and whole cell extracts were prepared which were subjected to sucrose density gradient centrifugation. The absorbance of each fraction at $260 \mathrm{~nm}$ was measured to determine the RNA content and to generate a profile. The peaks corresponding to (pre)ribosomal subunits are indicated. Proteins in each fraction (along with $10 \%$ input) were separated by SDSPAGE and analysed by western blotting with an anti-Flag antibody. 


\subsection{Discussion}

The pathway of ribosome assembly and maturation is characterized in much greater detail in yeast in comparison to that in humans. Hence, knowledge of ribosome biogenesis is largely derived from studies done in the yeast $S$. cerevisiae. Much of the knowledge on ribosome biogenesis in yeast is also applicable to higher eukaryotes due to a high degree of conservation of ribosome assembly factors and ribosomal proteins and similarities of the pre-rRNA processing pathways. However, a growing body of evidence indicates that the process of ribosome biogenesis has acquired greater complexity in humans in terms of additional pre-rRNA cleavage sites and ribosome assembly factors lacking yeast homologues. However, mechanistic details of ribosome biogenesis in humans is far from comprehensive with multiple ribosome assembly factors yet uncharacterized. Moreover, a group of genetic disorders, termed ribosomopathies, that are caused by mutations in genes encoding ribosomal proteins or assembly factors, further underscores the importance of a detailed understanding of the human ribosome assembly pathway.

In this study, the function of the putative RNA helicase DDX55, a homologue of yeast DEADbox protein Spb4, was analysed in the process of ribosome assembly in humans. In yeast, Spb4 has been reported to hydrolyse ATP in an RNA-dependent manner (Brüning et al., 2018) and in line with this, in vitro ATPase assays confirm the hydrolysis of ATP by DDX55 and we observed a lack of ATP hydrolysis by the mutant demonstrating that the observed activity can be attributed to DDX55. However, surprisingly, and in contrast to Spb4, the addition of RNA did not significantly stimulate ATP hydrolysis. On the one hand, this could reflect a difference between the mechanism of action of the yeast and human proteins, but on the other hand, it could arise due to possible non-specific co-purification of nucleic acids from E. coli during recombinant protein purification. This is further supported by the observation that DDX55 exhibited strong ATP hydrolysis activity even in the absence of an in vitro RNA substrate. Stringent purification protocol specifically optimized for the removal of bound nucleic acids from the protein fractions could be a possible strategy to counter this issue.

Our data further suggest that lack of DDX55 in human cells can lead to impaired ribosome biogenesis. Consistent with this, in vivo crosslinking data revealed the physical association of DDX55 with pre-LSU, and crosslinking to a specific 28S rRNA sequence. Analogous to the pre-ribosome binding site identified for yeast Spb4 (Brüning et al., 2018), DDX55 crosslinks to helices 62 and 63 of $28 S$ rRNA sequence suggesting that the helicase binding site is conserved from yeast to humans and further supporting the requirement for DDX55 for the maturation of pre-60S particles. In addition, similar to Spb4 in yeast, DDX55 does not crosslink to the $18 \mathrm{~S}$ rRNA sequence in human cells. However, depletion of DDX55 
resulted in mild defects in SSU biogenesis and while it is possible that DDX55 has a binding site in the 18S rRNA that was not identified by CRAC, it is likely that the effects of DDX55 depletion on 18S rRNA synthesis are indirect. We further demonstrated that DDX55 is a catalytically active enzyme and in order to demonstrate the requirement for the catalytic activity of DDX55 for ribosome biogenesis, RNAi-based rescue systems with cell lines expressing either wild-type or catalytically inactive DDX55 will be generated and used for analysing pre-rRNA processing.

In case of DEAH-box helicases, $\mathrm{N}$ - and $\mathrm{C}$ - terminal domains can serve as binding platforms for cofactors which can endow specificity to the intrinsically promiscuous helicase core. The OB-folds present in the conserved C-terminal domains of DEAH-box helicases are essential for binding to G-patch proteins which aid in the regulation as well as recruitment of helicases to their substrate RNAs. In the absence of such conserved domains in their C-terminal extensions, several DEAD-box helicases rely on their lysine rich C-terminal tail for targeting and/or tethering to their cognate RNA substrates. For example, the basic tail of yeast DEADbox helicase Mss 166 has been implicated in the binding of RNA and helps anchoring of the helicase to structured RNA substrates (Mohr et al., 2008). Interestingly, detailed investigation of the amino acid composition of DDX55 revealed the presence of a lysinerich C-terminal domain, which is conserved amongst eukaryotes. Consistent with a role in the late steps of ribosome biogenesis, full-length DDX55 was predominantly localised in the nucleoplasm and co-sediments with pre-60S complexes. However, upon truncation of the C-terminal tail, the protein was largely excluded from the nucleus and was unable to associate with pre-ribosomal complexes. The C-terminal domain of DDX55 is reminiscent of the basic tails of other helicases that tether them to their RNA substrates, raising the possibility that the lysine-rich tail of DDX55 could similarly contribute to its association with target RNA substrates. However, bioinformatics analysis of the C-terminal domain of DDX55 revealed the presence of a nuclear localization signal (NLS). The loss of the NLS could be responsible for the cytoplasmic mis-localisation of the truncated protein and the loss of association with pre-ribosomal complexes. On a molecular level, elements within the C-terminal tail are likely to have two distinct functions. They are implicated in promoting nuclear import and/or can facilitate the interaction of the helicase with substrate RNA, a function similar to yeast Mss116. To gain further insights and explain the effects observed upon tail deletion, an in vitro fluorescence anisotropy approach, comparing the RNA-binding ability of the full length and truncated proteins could differentiate between these two roles and determine if the C-terminal tail is directly implicated in RNA-binding. To further investigate the function of the C-terminal domain of DDX55, the predicted bipartite NLS could be exchanged for a minimal NLS and the RNA-binding ability of DDX55 and its effective recruitment to pre-ribosomal complexes could be tested. Furthermore, the 
contribution of C-terminal domain of DDX55 in the modulation of its catalytic activity in vivo also remains to be determined and methods such as in vitro ATPase assays together with RNAi-based rescue systems will be employed to address these open questions.

In yeast, Spb4 has been suggested to associate with pre-ribosomal particles in the nucleolus and is released relatively late prior to nuclear export of pre-60S particles (Brüning et al., 2018; de la Cruz et al., 1998). Recent reports suggest that Spb4 stably associates with helix 63 in 25S rRNA located at the base of ES27 which acts as a molecular hinge directing the position of a flexible arm that is involved in Arx1 binding to pre-60S particles. Binding of Spb4 likely promotes structural rearrangement enabling the recruitment of pre$60 S$ export factor Arx1 (Brüning et al., 2018). Additionally, Nog2, which acts as a placeholder for the export adaptor Nmd3 (Matsuo et al., 2014; Sloan et al., 2016) is also dependent on Spb4 for its recruitment to pre-60S particles (Talkish et al., 2012). These findings implicate Spb4 in helping establish the export competence of pre-60S particles. Interestingly, in humans, DDX55 associates with late pre-ribosomal particles and in contrast to $\mathrm{Spb4}$, it is predominantly excluded from the nucleolus (initial site of ribosome biogenesis) suggesting that the function or timing of this protein may differ in higher eukaryotes. Moreover, it remains to be determined if DDX55 is involved in preparing human pre-60S particles for export. This possibility is supported by the observation that DDX55 is localised predominantly in the nucleoplasm suggesting that it associates quite late with the preribosomal complexes during the assembly pathway. This potential function will be explored by employing fluorescence microscopy-based approaches monitoring the mis-localisation of GFP-tagged ribosomal subunits upon DDX55 depletion (Wild et al., 2010). Additionally, mass-spectrometry (MS) based compositional analysis of late pre-60S particles upon RNAimediated depletion of DDX55 could provide further insights into a potential role of DDX55 in promoting the recruitment or release of trans-acting biogenesis factors.

Taken together, the results so far suggest the requirement for DDX55 for maturation of LSU and further highlight the importance of its C-terminal tail region for interaction with preribosomal subunits. In the future, the various approaches described above will enable us to gain further mechanistic insights into the function of DDX55 in the context of LSU maturation and to understand the precise contribution of its basic C-terminal tail in regulating its function. 


\section{Chapter Five: Discussion}

\subsection{Distinct roles of RNA helicase in ribosome biogenesis}

Ribosomes are essential in all domains of life and the assembly of such large macromolecular complexes involves the action of various types of trans-acting biogenesis factors that mediate modification, cleavage, folding and restructuring of the pre-ribosomal complexes. Therefore, it is not surprising that RNA helicases, which play key roles in diverse aspects of RNA metabolism, are extensively involved in the process of ribosome biogenesis. In bacteria, ribosome assembly is best characterized in E. coli. Of the five nonessential RNA helicases known in E. coli, four of them have been reported to play a role in ribosome assembly (Martin et al., 2013). Studies have also shown that in bacterial strains, defects arising due to a loss of all five RNA helicases can be successfully alleviated by growing them in alternative conditions (Jagessar and Jain, 2010). However, in eukaryotes, the increased size and complexity of ribosomal subunits have led to the emergence of a more complex ribosome assembly pathway. Not surprisingly, most RNA helicases involved in ribosome biogenesis in eukaryotes are essential due to the key roles they play in remodelling pre-ribosomal particles and the remodelling events driven by them often serve as checkpoint during the assembly pathway. Ribosome biogenesis is best characterized in yeast (Woolford and Baserga, 2013) and so far, 21 RNA helicases have been shown to contribute to yeast ribosome biogenesis (Martin et al., 2013). Detailed molecular characterization of RNA helicases has revealed the diverse range of activities carried out by them in the context of ribosome biogenesis. Several RNA helicases have been implicated in facilitating the association or removal of snoRNAs from pre-ribosomal complexes (Bohnsack et al., 2008, 2009; Koš and Tollervey, 2005; Liang and Fournier, 2006; Martin et al., 2013; Watkins and Bohnsack, 2012). For example, the DEAD-box helicase Has1 has been implicated in the removal of U14 snoRNA in yeast (Brüning et al., 2018; Liang and Fournier, 2006). The well-characterized DEAH-box protein Prp43 has been suggested to play a direct role in the unwinding of several snoRNAs that modify a cluster of nucleotides in 25S rRNA sequence (Bohnsack et al., 2009). In yeast, Dhr1 (DHX37 in humans) associates with early pre-ribosomal particles and is suggested to trigger the release of U3 snoRNA (Sardana et al., 2015). The binding of U3 to pre-rRNA sequences is important for proper cleavage events within 5'ETS and ITS1, resulting in the formation of the mature 5 ' end of $18 \mathrm{~S}$ rRNA and separation of the pre-60S and pre-40S ribosomal subunits (Henras et al., 2008; Venema and Tollervey, 1995). The dissociation and release of the U3 snoRNA is also critical for the formation of the central pseudoknot, a key feature of the SSU (Henras et al., 2008). We observed that in human cells, DHX37 is required for 
the maturation of $18 \mathrm{~S}$ rRNA and plays a key role in the dissociation of U3 snoRNA from pre-ribosomal subunits, enabling the subsequent maturation of the SSU. Our CRAC data revealed direct interaction between DHX37 and the 3' hinge region of the U3 snoRNA, which is suggested to base-pair with 5'ETS sequences in the pre-rRNA. In accordance with this, expression of catalytically inactive $\mathrm{DHX} 37$ leads to impaired processing of the A' cleavage site within the 5' ETS resulting in the accumulation of aberrant pre-rRNAs.

Additionally, several RNA helicases are suggested to also contribute to the extensive prerRNA rearrangements that occur during ribosome assembly and which, for example, they facilitate efficient pre-rRNA cleavage, rRNA modification, or association/dissociation of different ribosomal proteins or biogenesis factors. For instance, in yeast, Kre33 is implicated in the acetylation of helix 34 and 35 within the 18S rRNA sequence. The conserved helicase domain of Kre33 was suggested to play a key role in the remodelling of 18S rRNA sequence in order for it to gain access and enable acetylation (Sharma et al., 2015). In addition to its role in the release of U14 snoRNA, Has1 has been reported to drive the remodelling of domain I of 25S rRNA enabling the incorporation of several RPs such as Rpl26, Rpl35 and Rpl37 (Brüning et al., 2018; Dembowski et al., 2013). The DEAD-box helicase Spb4 (DDX55 in humans), associates with pre-ribosomal complexes in the nucleolus and likely mediates structural rearrangement of the 25S pre-rRNA sequence (Brüning et al., 2018; de la Cruz et al., 1998). Interestingly and in contrast to Spb4, in humans, we observed DDX55 to be localized in the nucleoplasm suggesting its association with late-stage pre-ribosomal subunits. In vivo crosslinking data position both Spb4 and DDX55 in close proximity to helices 62 and 63 of $25 S / 28 S$ rRNA sequences, implying that this binding site is conserved between yeast and humans. In yeast, remodelling activity of Spb4 at helices 62 and 63 of $25 S$ rRNA has been suggested to promote the recruitment of the export adaptor Arx1. Interestingly, EBP1, the human homologue of Arx1, has been reported to associate with pre-60S particles. However, the precise binding sites of EBP1 on pre-ribosomal subunits is yet to be identified (Squatrito et al., 2004). It remains to be seen if DDX55 plays a role in the recruitment of export factors in the context of LSU biogenesis in humans. Although assumed to be highly conserved, an increasing body of evidence has highlighted key differences in the biogenesis pathway of yeast and human ribosomes implying that ribosome biogenesis has evolved to be more complex in humans. Hence, the list of assembly factors involved in ribosome biogenesis in humans is likely still incomplete (Sloan et al., 2013a; Tafforeau et al., 2013; Wild et al., 2010). Several RNA helicases that were previously functionally characterized in yeast, were shown to fulfil similar tasks in humans (Choudhury et al., 2019; Sekiguchi et al., 2006). However, there are now also examples of helicases which either lack a yeast homologue (DDX21) or play different roles in higher eukaryotes (DHX15) (Memet et al., 2017; Sloan et al., 2015). Moreover, RNAi-mediated 
screens have identified several RNA helicases which are required for SSU and LSU biogenesis but are yet to be characterized in detail (Tafforeau et al., 2013). Thus, the molecular functions of most RNA helicases involved in ribosome biogenesis in higher eukaryotes are not well-understood and the emergence of ribosomopathies further emphasizes the need to characterize the human ribosome assembly pathway in detail.

\subsection{Division of labour: DEAH-box versus DEAD-box helicases}

Various aspects of gene expression such as pre-mRNA splicing or ribosome biogenesis require the contribution of RNA helicases belonging to either the DEAD-box or DEAH-box family of proteins. As observed in certain pathways, there seems to be a clear division of labour between proteins belonging to the two helicase families in assisting distinct assembly/disassembly steps, implying the requirement for different modes of strand separation. This is perhaps most evident in yeast pre-mRNA splicing, which requires the action of eight helicases which fall into the categories of DEAD-box, DEAH-box and Ski2like helicases (Cordin and Beggs, 2013). The pre-catalytic steps are assisted by DEAD-box helicases which act as local 'unwindases' and typically bind to short duplex regions in an ATP-dependent manner. For example, during the first steps of splicing, the DEAD-box proteins Sub2 and Prp5 are implicated in branch point recognition by U2 snRNP by mediating local structural rearrangements. Prp5 is suggested to facilitate the transition of U2 snRNP between alternate conformation by restructuring an 8 bp helix. Subsequently, the DEAD-box helicase Prp28 is important for the unwinding of a 6 bp helix formed between the U1 snRNA and the 5' splice site, resulting in the release of the U1 snRNP. In contrast, DEAH-box helicases being processive enzymes, promote extensive structural rearrangements involving long-range interactions in the downstream catalytic steps (Gilman et al., 2017). For example, the DEAH-box helicase Prp2 is implicated in displacement of proteins from the branch point by translocating through the pre-mRNA in a 3'-5' manner. Likewise, a directional 3'-5' translocation has also been suggested for Prp22, resulting in the release of spliced mRNA. Lastly, the multi-functional DEAH-box helicase Prp43 promotes extensive re-structuring events leading to release of the intron lariat and recycling of the snRNPs (Cordin and Beggs, 2013; Fica and Nagai, 2017). In addition, DEAD-box proteins involved in local strand unwinding and duplex destabilization are key players in remodelling of carefully structured RNP complexes such as pre-ribosomes. Local destabilization within a layered RNP complex leads to large-scale structural rearrangements required for ribosome assembly and maturation, making DEAD-box helicases excellent candidates for this role (Jankowsky and Fairman, 2007). For instance, several DEAD-box helicases are suggested to promote unwinding of snoRNA-pre-rRNA base-pairing by mediating local unwinding (Martin et al., 2014). 
Interestingly, although there is typically a clear demarcation between the molecular mechanisms of action of DEAD-box and DEAH-box helicases, studies have shown that there are a few exceptions. The DEAH-box helicase Prp43, in yeast, is exceptionally versatile, playing diverse roles in ribosome biogenesis and pre-mRNA splicing (Jarmoskaite and Russell, 2014). DEAH-box helicases are typified as unidirectional enzymes that bind to 3' single stranded sequences and translocate in a strict 3'-5' manner. In vitro studies have demonstrated that Prp43 does not require a 3' extension for unwinding of RNA and hence, can function in a manner similar to DEAD-box helicases by being directly recruited to structured, double stranded RNA sequences (Guenther and Jankowsky, 2009). Likewise, the yeast DEAH-box helicase Dhr1 was suggested to unwind RNA duplexes in a manner reminiscent of DEAD-box helicases. In vitro unwinding assays with ATP binding or hydrolysis mutants of Dhr1 indicated that the unwinding activity is dependent on ATP binding and that hydrolysis of bound ATP was only important for product release and dissociation of the helicase from the substrate (Sardana et al., 2015). This is in contrast to typical DEAH-box helicases as they rely on successive cycles of ATP hydrolysis for processive strand displacement but similar to the classical mechanism adopted by DEADbox helicases for unwinding short duplexes. Recent structural insights provide mechanistic details of the RNA-dependent ATPase activity of the human homologue of Dhr1, DHX37. A crystal structure of DHX37 in the presence of a bound single stranded RNA substrate revealed that ATP binding to the conserved motifs within the helicase core domains induces a conformational shift resulting in formation of the 'closed' active conformation. This leads to a directional shift with the helicase engaging with the next 5 ' nucleotide in the substrate while releasing one base from the 3' side. Upon ATP hydrolysis, the helicase switches back to its inactive 'open' conformation and can associate with the next 5 ' terminal nucleotide. Hence, DHX37 is able to translocate in a 3'-5' unidirectional manner and ATP binding but not hydrolysis was suggested to provide the power stroke for translocation (Boneberg et al., 2019).

CRAC analysis of yeast DEAD-box helicase Spb4, identified two distinct crosslinking sites on the 25S rRNA sequence. However, crosslinking sites of helicases identified by approaches like CRAC do not necessarily represent the contact point of the active core of the helicase, but can also represent contacts formed by other regions of the protein. This can often be the case as it is likely that the active site makes only transient interactions with the RNA that are less likely to be captured by CRAC. Structural analysis of pre-ribosomal particles containing the helicase are required in order to identify RNA sequences that are directly contacted by the active site of the helicase. Dimethyl sulphate (DMS) structure probing experiments, aimed at uncoupling the functional relevance of the two sites identified in the case of Spb4, suggested that helices $62-63$ are likely to serve as a binding platform 
for the helicase whereas helix 101 may represent the remodelling site. Mapping of the CRAC data onto the 3D structure of pre-LSU particles showed that the two binding sites are present on the same face of the ribosome, albeit somewhat specially separated (Brüning et al., 2018). In vivo, however, DEAD-box helicases have been suggested to promote local strand separation raising the question of how DEAD-box helicases such as Spb4 are able to promote duplex destabilization at regions spatially distant from their binding sites. For long RNA substrates, some DEAD-box helicases have been shown to tether securely to RNA sequences spatially distinct from their remodelling targets, thereby enabling multiple rounds of helix capture, strand separation and ATP hydrolysis in the vicinity of their binding site. For example, yeast helicase Mss116 possess an arginine rich C-terminal extension that facilitates its anchoring onto the RNA substrate in order to perform multiple rounds of ATP hydrolysis (Mohr et al., 2008). Likewise, Spb4 contains numerous basic amino acid residues at the C-terminal tail extension, suggesting a similar mechanism of action. Interestingly, our CRAC data reveal a distinct binding site for DDX55 within the 28S rRNA sequence. The functional relevance of DDX55 binding to 28S rRNA sequence is still to be determined. However, taken together with its nucleoplasmic localisation, it is possible that DDX55 plays a role in late stages of pre-60S maturation as seen in the case of Spb4. Interestingly, detailed sequence characterization of DDX55 revealed the presence of Cterminal tail containing clusters of basic amino acid residues. Deletion of the C-terminal tail, downstream of the conserved core domain leads to mis-localization of the protein to the cytoplasm, thereby resulting in a loss of association with pre-60S particles as observed in sucrose density gradient experiments. In vitro studies such as fluorescence anisotropy experiments will provide clearer insights into the contribution of $\mathrm{C}$-terminal tail in the binding and subsequent association of DDX55 with pre-ribosomal complexes.

Additionally, for several well-characterized DEAD-box proteins that display conventional non-processive methods of duplex unwinding, association with dedicated cofactors has been shown to drastically influence their properties. For example, in humans, the DEADbox helicase elF4A has been suggested to exhibit processive cycles of duplex unwinding in association with its partner proteins elF4G and elF4B (García-García et al., 2015). It is also likely that individual helicases have evolved specific properties distinctive from the classical enzymes of their respective families in order to perform a specialized task in vivo. Detailed biochemical and structural characterization of individual RNA helicases from the DEAD-box and DEAH-box families will therefore likely provide new insights into their precise mechanisms of action. 


\subsection{Regulation of RNA helicases}

The conserved helicase core domains of DEAD-box proteins and DEAH-box proteins interact with the sugar phosphate backbone of their RNA substrates, thereby exhibiting an intrinsic lack of specificity. However, the existence of multi-functional helicases such as Prp43 and elF4A highlight the ability of these enzymes to bind to specific RNA and/or RNP targets in the complex cellular environment. The $\mathrm{N}$ - and $\mathrm{C}$-terminal auxiliary domains extending beyond the RecA-like domains have been implicated in influencing the target recognition and activity of these helicases. The $\mathrm{C}$-terminal extensions of DEAD-box proteins is not conserved and often varies within the helicase family. In contrast, DEAH-box proteins possess a highly conserved C-terminus consisting of $\mathrm{OB}$-fold, $\mathrm{WH}$ and the ratchet domain, which are implicated in substrate recognition and regulation through association with cofactors such as the G-patch family of proteins (Robert-Paganin et al., 2015). Alongside helicase cofactors such as the MIF4G and G-patch proteins that form family groups, several proteins that do not share any clear similarities with other cofactors and have evolved to influence the activity of specific helicases. For example, in yeast, the unwinding activity of DEAH-box helicase Dhr1 is stimulated by the 40S biogenesis factor Utp14. Additionally, Utp14 along with rRNA methyltransferase Bud23 has been reported to be required for the association of Dhr1 with the pre-ribosomal complexes (Zhu et al., 2016). Once recruited to pre-ribosomes, Dhr1 and Utp14 act synergistically leading to the efficient dissociation of U3 snoRNA from the pre-rRNA sequences (Zhu et al., 2016). Our data indicate a direct, RNAindependent interaction between UTP14A and DHX37 in humans, suggesting that this interaction is conserved between yeast and humans. In vitro ATPase assays further demonstrate that UTP14A stimulates the ATPase activity of DHX37 and RNAi-mediated depletion of UTP14A leads to accumulation of U3 snoRNA on pre-ribosomal complexes. This is in contrast to a previous observation that in yeast, Utp14 stimulates the unwinding activity of Dhr1 without altering its ATPase activity (Zhu et al., 2016), but is in accordance with recent reports investigating the mechanistic details of UTP14 dependent activation of DHX37 (Boneberg et al., 2019). Although the molecular mechanisms of Utp14/UTP14A mediated stimulation of Dhr1/DHX37 may therefore have diverged during evolution, the physiological function of the helicase and its cofactor seems to be conserved in higher eukaryotes. Fluorescence polarization assays used for monitoring RNA binding have further revealed the precise mechanism of DHX37 stimulation by UTP14A (Boneberg et al., 2019). A conserved motif in the central region of UTP14A has been implicated in the stimulation of RNA-dependent ATPase activity of DHX37 by enhancing the ATP-dependent RNA-binding ability of DHX37. Furthermore, structural studies of $\mathrm{N}$-terminal truncated $\mathrm{DHX} 37$ reveal the presence of a unique alpha-helical C-terminal domain, which has not been previously observed in other proteins. Deletion of the C-terminus results in the loss of DHX37 
association with UTP14A, indicating that this domain is crucial for efficient interaction with UTP14A. Moreover, fluorescence-based reporter assays indicated that although the Cterminal domain is not required for the nuclear import of DHX37, it plays an important role in the biogenesis of $40 \mathrm{~S}$ ribosomal subunit in vivo (Boneberg et al., 2019).

Additionally, a diverse range of helicases are also regulated in cis by the action of auxiliary domains. Prominent examples include the yeast DEAD-box protein Mss116 and its Neurospora crassa orthologue CYT-19 wherein the basic C-terminal tail is implicated in the binding to RNA substrates. Interestingly, the proposed binding platform of Spb4 constitutes of a helical structure reminiscent of the secondary structure bound by the C-terminus of Mss116 (Brüning et al., 2018), implying a similar mode of recruitment. Our data suggest that the basic amino acid-rich C-terminal domain of DEAD-box protein DDX55 might also play a role in its recruitment to the pre-ribosomal subunits. However, it remains to be determined if the basic residues within the C-terminal domain directly contribute to the recruitment and regulation of DDX55. To determine the involvement of a possible transacting cofactor, immunoprecipitation experiments followed by mass-spectrometry could provide additional information regarding proteins that contribute to spatial and temporal regulation of the catalytic activity of DDX55. Alternatively, it is also possible that DDX55 is recruited and its activity is regulated by its basic C-terminal tail without the involvement of trans-acting effector proteins. Local duplex destabilization mediated by binding of a helicase within the context of a large RNP particle, like a pre-ribosome, can often lead to dissociation of bases downstream of the binding site leading to restructuring of the RNP complex. For example, in the case of Spb4, the binding site is suggested to be spatially separated from the site of action. However, the binding of Spb4 may also result in a structural rearrangement within its site of action, allowing the association of export adaptor protein Arx1 (Brüning et al., 2018). This phenomenon is analogous to the 'butterfly effect' whereby a miniscule localized change in a complex system ultimately manifests in a large effect. Unfortunately, the precise RNA targets of many DEAD and DEAH-box helicases are still unknown in humans. In vivo crosslinking based approaches such as CRAC are now increasingly being employed to identify targets of these helicases. Additionally, the newly available cryo-EM structures of large RNPs such as spliceosomes and pre-ribosomes significantly help identifying the targets of several helicases. Identifying RNA helicase cofactors remains extremely challenging, especially for proteins that function in large RNPs like pre-ribosomes, however, the development of improved protein-protein crosslinking and proximity labelling techniques will likely lead to the identification of additional RNA helicase cofactors in the future. Identification of target RNA substrates as well as their interactome will lead to a more comprehensive understanding of the regulatory networks governing the action of helicases in vivo. 


\subsection{Dynamics of RNA helicase association with pre-ribosomes.}

RNA helicases associate with pre-ribosomal subunits and through their diverse range of functions, mediate remodelling of these complexes. However, it is often difficult to dissect the dynamics of helicase association with pre-ribosomal complexes. In humans, DHX37 is observed to associate with early pre-ribosomal particles where it functions in the removal of U3 snoRNP. Expression of catalytically inactive DHX37 leads to impaired processing at the A' cleavage site located within the 5' ETS region, likely reflecting the failure to release U3 snoRNA upon DHX37 depletion. Our studies further suggest that the association of DHX37 to early pre-ribosomal complexes is monitored by various quality control pathways and failure to recruit DHX37 along with the 40 S biogenesis factors DIMT1L and TSR1 result in degradation of the pre-ribosomes. Interestingly, complementation assays further reveal that the catalytic activity of $\mathrm{DHX} 37$ is necessary for the processing of $21 \mathrm{~S}$ pre-rRNA to generate $18 \mathrm{~S}-\mathrm{E}$ pre-rRNA during the late stages of ribosome biogenesis. Taken together, our results indicate that $\mathrm{DHX} 37$ is required for the early and late steps of 405 ribosomal biogenesis. A possible explanation coupling the early and late stage defects could be that the failure to release U3 snoRNA from early pre-ribosomal particles leads to defects in late stages of pre-rRNA processing. Additionally, in humans, the precise timing of U3 snoRNA release is not yet known. Alternatively, the defects in the late stages of pre-40S maturation can likely reflect an additional role for DHX37 in the pre-rRNA processing of 21S, independent of its function in the release of U3 snoRNA in the early steps of biogenesis. This raises an interesting question regarding the temporal regulation of $\mathrm{DHX} 37$. It is yet to be determined if $\mathrm{DHX} 37$ associates with the ribosome at an early stage and is released only after the successful processing of $21 \mathrm{~S}$ to $18 \mathrm{~S}-\mathrm{E}$ or upon efficient dissociation of U3 snoRNA, or whether DHX37 is released from the pre-ribosomal subunits after U3 release and then re-associates during late-steps of ribosome biogenesis. Our data further suggest that UTP14A acts together with DHX37 in promoting the release of U3 snoRNA. Immunoprecipitation followed by mass-spectrometry analysis of the DHX37 interactome may reveal the presence of additional cofactors of DHX37 that could regulate the activity of DHX37 during later steps of biogenesis. These open questions regarding the timing of DHX37 association and link between the early and late stage defects can probably be best addressed by structural analysis of the helicase in association with pre-ribosomal complexes isolated during different stages of biogenesis. In addition, MS analyses of preribosomal particles containing $\mathrm{DHX} 37$ can also provide information regarding the timing of its association. As DHX37, similar to Dhr1, does not stably associate with pre-ribosomal complexes, our in vivo crosslinking data did not reveal its binding site on the pre-rRNA.

Performing CRAC experiments using catalytically inactive mutant of DHX37 could possibly 
lead to a more stable association of the helicase with the pre-ribosomal complexes, thereby providing additional information regarding the pre-rRNA target sites of DHX37.

In contrast to DHX37, the DEAD-box helicase DDX55, binds to pre-ribosomes during the late steps of ribosome biogenesis. In vivo crosslinking data suggest its direct association with the 28S rRNA sequence. The DDX55 binding site is observed to be conserved between yeast and humans and the late association of DDX55 with pre-60S complexes could indicate a possible role in establishing export competence of pre-60S complexes. While it is likely that late-acting RNA helicases like DDX55 remodel RNA sequences present on the surface of pre-ribosomal particles, it is also possible that they influence RNA structures buried deep within the RNP complex. Recent reports have proposed a 'winching' model for DEAH-box helicases that may explain the mechanism by which helicases are able to gain access to their target substrates within the context of large RNPs. Based on biochemical studies from yeast DEAH-box helicases Prp16 and Prp22, it was proposed that helicases can load onto 3' exposed extensions and upon encountering a block in translocation, they are able to pull on the RNA strand. This results in disruption in base-pairing without the helicase accessing the buried regions within the RNP (Semlow et al., 2016). This model highlights how DEAH-box helicases can act from a distance without having to translocate through their substrates and it is likely that this mechanism can also be employed by other members of the DEAH-box family of helicases. In contrast, DEAD-box helicases need direct association with or in the vicinity their duplex substrate and are not capable of acting from a distance as the winching model proposes. Auxiliary domains consisting of flexible basic C-terminal tail extensions or RNA recognition motifs in some cases have been proposed to anchor the helicase at an exposed region proximal to the remodelling site within the RNP complex. Once securely anchored to the RNA substrate, the helicase core gains access to the target site and remodels it (Diges and Uhlenbeck, 2001; Karginov et al., 2005; Mohr et al., 2008). An alternative possibility could be via the assistance of various cofactors that can help in the recruitment of helicases within the context of an RNP complex. Alternatively, the remodelling sites, if present on the surface of the pre-ribosomes, are easily accessible to DEAD-box helicases for binding.

Another complexity that arises as a result of RNA helicases acting in the context of large RNPs is the difficulty in distinguishing the direct consequences of RNA helicase action from indirect effects. A key example is the case of RNA helicases mediating snoRNA release from pre-ribosomal complexes. Depletion of more than one helicase can lead to the accumulation of a specific snoRNA on pre-ribosomes (Bohnsack et al., 2008; Koš and Tollervey, 2005; Liang and Fournier, 2006; Martin et al., 2014). For example, in yeast, DEAD-box helicases Dpb4 and Has1 have both been implicated in the release of U14 snoRNA from pre-ribosomes. U14 is required for guiding the 2'-O-methylation in 18S rRNA 
and also for mediating pre-rRNA rearrangement required for processing (Dunbar and Baserga, 1998; Liang and Fournier, 1995). CRAC analyses have established the physical association of Has1 with U14, implying the direct role of Has 1 in the release of U14 (Brüning et al., 2018; Liang and Fournier, 2006). However, so far, there is no direct evidence of Dbp4 interaction with Has1. Moreover, U14 was not found to be present in particles associated with Dbp4 (Soltanieh et al., 2015). Hence, it is likely that depletion of Dpb4 leads to remodelling events within the pre-ribosomal subunit, which indirectly retains $U 14$. The yeast RNA helicase Rok1 is implicated in the unwinding and release of snR30 from pre-ribosomal complexes (Bohnsack et al., 2008). In vivo crosslinking data revealed that Rok1 associates with snR30 and promotes direct unwinding of snR30-pre-rRNA base-pairing (Martin et al., 2014). The strongest evidence indicating a direct role for a helicase in unwinding snoRNApre-rRNA base-pairing is supported by the data reported for Prp43. Crosslinking data revealed that Prp43 contacts the snoRNAs as well as their base-pairing sites on the prerRNAs sequences indicating a direct role in unwinding and release of snoRNAs (Bohnsack et al., 2009). Likewise, in humans, DDX51 is required for the displacement of U8 snoRNA from pre-ribosomes. However, as there is no evidence of direct binding between U8 and DDX51, it is also possible that instead of being directly involved in the unwinding of a U8 snoRNA-pre-rRNA duplex, DDX51 mediates structural rearrangements in distant areas of the pre-ribosome that indirectly lead to U8 snoRNA release (Srivastava et al., 2010). In yeast, Dhr1 crosslinks to the 5' region of the U3 snoRNA sequence which associates with the $18 \mathrm{~S}$ rRNA sequence. However, Dhr1 was also reported to promote the unwinding of a duplex formed between 3' region of U3 and the 5'ETS of pre-rRNA. Hence, it is yet to be determined if Dhr1 acts to unwind both these duplexes in vivo or the disruption of one duplex is sufficient to promote the dissociation of U3 from pre-ribosomes (Sardana et al., 2015). Our CRAC data suggest a direct physical contact between 3' hinge region of U3 snoRNA that is predicted to base-pair with 5'ETS region and DHX37, implying that DHX37 may have a direct role in the dissociating U3 snoRNA form the pre-ribosomal subunits. The development of methods that allow visualisation of RNA helicases within pre-ribosomes, and structural analyses of helicases in the context of RNP complexes will be important approaches for better dissecting the precise roles of RNA helicases in vivo. 


\section{Bibliography}

Abaeva, I.S., Marintchev, A., Pisareva, V.P., Hellen, C.U.T., and Pestova, T. V (2011). Bypassing of stems versus linear base-by-base inspection of mammalian mRNAs during ribosomal scanning. EMBO J. 30, 115-129.

Abdelhaleem, M., Maltais, L., and Wain, H. (2003). The human DDX and DHX gene families of putative RNA helicases. Genomics 81, 618-622.

Ahmad, Y., Boisvert, F.-M., Gregor, P., Cobley, A., and Lamond, A.I. (2009). NOPdb: Nucleolar Proteome Database--2008 update. Nucleic Acids Res. 37, D181-4.

Aitken, C.E., and Lorsch, J.R. (2012). A mechanistic overview of translation initiation in eukaryotes. Nat. Struct. Mol. Biol. 19, 568-576.

Alexandrov, A., Colognori, D., and Steitz, J.A. (2011). Human elF4AIII interacts with an elF4G-like partner, NOM1, revealing an evolutionarily conserved function outside the exon junction complex. Genes Dev. 25, 1078-1090.

Alexandrov, A., Colognori, D., Shu, M.-D., and Steitz, J.A. (2012). Human spliceosomal protein CWC22 plays a role in coupling splicing to exon junction complex deposition and nonsense-mediated decay. PNAS 109, 21313-21318.

Ameismeier, M., Cheng, J., Berninghausen, O., and Beckmann, R. (2018). Visualizing late states of human $40 S$ ribosomal subunit maturation. Nature $558,249-253$.

Andersen, J.S., Lyon, C.E., Fox, A.H., Leung, A.K.L., Lam, Y.W., Steen, H., Mann, M., and Lamond, A.I. (2002). Directed proteomic analysis of the human nucleolus. Curr. Biol. 12, 111.

Andreou, A.Z., and Klostermeier, D. (2014). elF4B and elF4G Jointly Stimulate elF4A ATPase and Unwinding Activities by Modulation of the elF4A Conformational Cycle. J. Mol. Biol. 426, 51-61.

Anger, A.M., Armache, J.-P., Berninghausen, O., Habeck, M., Subklewe, M., Wilson, D.N., and Beckmann, R. (2013). Structures of the human and Drosophila $80 \mathrm{~S}$ ribosome. Nature 497, 80-85. 
Ansel, K.M., Pastor, W.A., Rath, N., Lapan, A.D., Glasmacher, E., Wolf, C., Smith, L.C., Papadopoulou, N., Lamperti, E.D., Tahiliani, M., et al. (2008). Mouse Eri1 interacts with the ribosome and catalyzes 5.8S rRNA processing. Nat. Struct. Mol. Biol. 15, 523-530.

Aravind, L., and Koonin, E. V (1999). Novel predicted RNA-binding domains associated with the translation machinery. J. Mol. Evol. 48, 291-302.

Arcus, V. (2002). OB-fold domains: a snapshot of the evolution of sequence, structure and function. Curr. Opin. Struct. Biol. 12, 794-801.

Armistead, J., Khatkar, S., Meyer, B., Mark, B.L., Patel, N., Coghlan, G., Lamont, R.E., Liu, S., Wiechert, J., Cattini, P.A., et al. (2009). Mutation of a Gene Essential for Ribosome Biogenesis, EMG1, Causes Bowen-Conradi Syndrome. Am. J. Hum. Genet. 84, 728-739.

Bai, D., Zhang, J., Li, T., Hang, R., Liu, Y., Tian, Y., Huang, D., Qu, L., Cao, X., Ji, J., et al. (2016). The ATPase hCINAP regulates 18S rRNA processing and is essential for embryogenesis and tumour growth. Nat. Commun. 7, 12310.

Banroques, J., Cordin, O., Doère, M., Linder, P., and Tanner, N.K. (2008). A conserved phenylalanine of motif IV in superfamily 2 helicases is required for cooperative, ATPdependent binding of RNA substrates in DEAD-box proteins. Mol. Cell. Biol. 28, 3359-3371.

Barandun, J., Chaker-Margot, M., Hunziker, M., Molloy, K.R., Chait, B.T., and Klinge, S. (2017). The complete structure of the small-subunit processome. Nat. Struct. Mol. Biol. 24, 944-953.

Beltrame, M., and Tollervey, D. (1995). Base pairing between U3 and the pre-ribosomal RNA is required for $18 \mathrm{~S}$ rRNA synthesis. EMBO J. 14, 4350-4356.

Ben-Shem, A., Garreau de Loubresse, N., Melnikov, S., Jenner, L., Yusupova, G., and Yusupov, M. (2011). The Structure of the Eukaryotic Ribosome at 3.0 A Resolution. Science (80-. ). 334, 1524-1529.

Bohnsack, K.E., and Bohnsack, M.T. (2019). Uncovering the assembly pathway of human ribosomes and its emerging links to disease. EMBO J. e100278.

Bohnsack, M.T., Kos, M., and Tollervey, D. (2008). Quantitative analysis of snoRNA 
association with pre-ribosomes and release of snR30 by Rok1 helicase. EMBO Rep. 9 , $1230-1236$.

Bohnsack, M.T., Martin, R., Granneman, S., Ruprecht, M., Schleiff, E., and Tollervey, D. (2009a). Prp43 Bound at Different Sites on the Pre-rRNA Performs Distinct Functions in Ribosome Synthesis. Mol. Cell 36, 583-592.

Bohnsack, M.T., Martin, R., Granneman, S., Ruprecht, M., Schleiff, E., and Tollervey, D. (2009b). Prp43 Bound at Different Sites on the Pre-rRNA Performs Distinct Functions in Ribosome Synthesis. Mol. Cell 36, 583-592.

Bohnsack, M.T., Tollervey, D., and Granneman, S. (2012). Identification of RNA Helicase Target Sites by UV Cross-Linking and Analysis of cDNA. Methods Enzymol. 511, 275-288.

Boneberg, F.M., Brandmann, T., Kobel, L., van den Heuvel, J., Bargsten, K., Bammert, L., Kutay, U., and Jinek, M. (2019). Molecular mechanism of the RNA helicase DHX37 and its activation by UTP14A in ribosome biogenesis. RNA 25, 685-701.

Boon, K.-L., Auchynnikava, T., Edwalds-Gilbert, G., Barrass, J.D., Droop, A.P., Dez, C., and Beggs, J.D. (2006). Yeast Ntr1/Spp382 Mediates Prp43 Function in Postspliceosomes. Mol. Cell. Biol. 26, 6016-6023.

Booy, E.P., McRae, E.K.S., Howard, R., Deo, S.R., Ariyo, E.O., Dzananovic, E., Meier, M., Stetefeld, J., and McKenna, S.A. (2016). RNA Helicase Associated with AU-rich Element (RHAU/DHX36) Interacts with the 3'-Tail of the Long Non-coding RNA BC200 (BCYRN1). J. Biol. Chem. 291, 5355-5372.

Boria, I., Garelli, E., Gazda, H.T., Aspesi, A., Quarello, P., Pavesi, E., Ferrante, D., Meerpohl, J.J., Kartal, M., Da Costa, L., et al. (2010). The ribosomal basis of diamondblackfan anemia: mutation and database update. Hum. Mutat. 31, 1269-1279.

Bortoluzzi, S., D’alessi, F., Romualdi, C., and Danieli, G.A. (2001). Differential expression of genes coding for ribosomal proteins in different human tissues.

Bourgeois, C.F., Mortreux, F., and Auboeuf, D. (2016). The multiple functions of RNA helicases as drivers and regulators of gene expression. Nat. Rev. Mol. Cell Biol. 17, 426438. 
Brüning, L., Hackert, P., Martin, R., Davila Gallesio, J., Aquino, G.R.R., Urlaub, H., Sloan, K.E., and Bohnsack, M.T. (2018). RNA helicases mediate structural transitions and compositional changes in pre-ribosomal complexes. Nat. Commun. 9, 5383.

Büttner, K., Nehring, S., and Hopfner, K.-P. (2007). Structural basis for DNA duplex separation by a superfamily-2 helicase. Nat. Struct. Mol. Biol. 14, 647-652.

Caruthers, J.M., and McKay, D.B. (2002). Helicase structure and mechanism. Curr. Opin. Struct. Biol. 12, 123-133.

Chen, S.S., and Williamson, J.R. (2013). Characterization of the ribosome biogenesis landscape in E. coli using quantitative mass spectrometry. J. Mol. Biol. 425, 767-779.

Chen, W., Shulha, H.P., Ashar-Patel, A., Yan, J., Green, K.M., Query, C.C., Rhind, N., Weng, Z., and Moore, M.J. (2014a). Endogenous U2·U5.U6 snRNA complexes in S. pombe are intron lariat spliceosomes. RNA 20, 308.

Chen, Y.-L., Capeyrou, R., Humbert, O., Mouffok, S., Kadri, Y.A., Lebaron, S., Henras, A.K., and Henry, Y. (2014b). The telomerase inhibitor Gno1p/PINX1 activates the helicase Prp43p during ribosome biogenesis. Nucleic Acids Res. 42, 7330-7345.

Chen, Y., Potratz, J.P., Tijerina, P., Del Campo, M., Lambowitz, A.M., and Russell, R. (2008). DEAD-box proteins can completely separate an RNA duplex using a single ATP. Proc. Natl. Acad. Sci. U. S. A. 105, 20203-20208.

Cheng, J., Kellner, N., Berninghausen, O., Hurt, E., and Beckmann, R. (2017). 3.2-Åresolution structure of the $90 \mathrm{~S}$ preribosome before A1 pre-rRNA cleavage. Nat. Struct. Mol. Biol. 24, 954-964.

Choesmel, V., Bacqueville, D., Rouquette, J., Noaillac-Depeyre, J., Fribourg, S., Cretien, A., Leblanc, T., Tchernia, G., Da Costa, L., and Gleizes, P.-E. (2006). Impaired ribosome biogenesis in Diamond-Blackfan anemia. Blood 109, 1275-1283.

Choudhury, P., Hackert, P., Memet, I., Sloan, K.E., and Bohnsack, M.T. (2019). The human RNA helicase DHX37 is required for release of the U3 snoRNP from pre-ribosomal particles. RNA Biol. 16, 1-15. 
Christian, H., Hofele, R. V, Urlaub, H., and Ficner, R. (2014). Insights into the activation of the helicase Prp43 by biochemical studies and structural mass spectrometry. Nucleic Acids Res. 42, 1162-1179.

Ciganda, M., and Williams, N. (2011). Eukaryotic 5S rRNA biogenesis. Wiley Interdiscip. Rev. RNA 2, 523-533.

Collins, R., Karlberg, T., Lehtiö, L., Schütz, P., van den Berg, S., Dahlgren, L.-G., Hammarström, M., Weigelt, J., and Schüler, H. (2009). The DEXD/H-box RNA helicase DDX19 is regulated by an \{alpha\}-helical switch. J. Biol. Chem. 284, 10296-10300.

Cordin, O., and Beggs, J.D. (2013). RNA helicases in splicing. RNA Biol. 10, 83-95.

Da Costa, L., Narla, A., and Mohandas, N. (2018). An update on the pathogenesis and diagnosis of Diamond-Blackfan anemia. F1000Research 7.

Couté, Y., Kindbeiter, K., phane Belin, S., gis Dieckmann, R., Duret, L., Bezin, L., Sanchez, J.-C., and Diaz, J.-J. (2008a). ISG20L2, a Novel Vertebrate Nucleolar Exoribonuclease Involved in Ribosome Biogenesis* $\square \mathrm{S}$.

Couté, Y., Kindbeiter, K., Belin, S., Dieckmann, R., Duret, L., Bezin, L., Sanchez, J.-C., and Diaz, J.-J. (2008b). ISG20L2, a Novel Vertebrate Nucleolar Exoribonuclease Involved in Ribosome Biogenesis. Mol. Cell. Proteomics 7, 546-559.

Danilova, N., and Gazda, H.T. (2015). Ribosomopathies: how a common root can cause a tree of pathologies. Dis. Model. Mech. 8, 1013-1026.

Dardenne, E., Polay Espinoza, M., Fattet, L., Germann, S., Lambert, M.-P., Neil, H., Zonta, E., Mortada, H., Gratadou, L., Deygas, M., et al. (2014). RNA Helicases DDX5 and DDX17 Dynamically Orchestrate Transcription, miRNA, and Splicing Programs in Cell Differentiation. Cell Rep. 7, 1900-1913.

Daugeron, M.-C., and Linder, P. (2001). Characterization and mutational analysis of yeast Dbp8p, a putative RNA helicase involved in ribosome biogenesis. Nucleic Acids Res. 29, 1144-1155.

Decatur, W.A., and Fournier, M.J. (2002). rRNA modifications and ribosome function. 
Trends Biochem. Sci. 27, 344-351.

Dembowski, J.A., Ramesh, M., McManus, C.J., and Woolford, J.L. (2013). Identification of the binding site of Rlp7 on assembling 60S ribosomal subunits in Saccharomyces cerevisiae. RNA 19, 1639-1647.

Diges, C.M., and Uhlenbeck, O.C. (2001). Escherichia coli DbpA is an RNA helicase that requires hairpin 92 of 23S rRNA. EMBO J. 20, 5503-5512.

Donati, G., Peddigari, S., Mercer, C.A., and Thomas, G. (2013). 5S Ribosomal RNA Is an Essential Component of a Nascent Ribosomal Precursor Complex that Regulates the Hdm2-p53 Checkpoint. Cell Rep. 4, 87-98.

Dunbar, D.A., and Baserga, S.J. (1998). The U14 snoRNA is required for 2'-O-methylation of the pre-18S rRNA in Xenopus oocytes. RNA 4, 195-204.

Dutca, L.M., Gallagher, J.E.G., and Baserga, S.J. (2011). The initial U3 snoRNA:pre-rRNA base pairing interaction required for pre-18S rRNA folding revealed by in vivo chemical probing. Nucleic Acids Res. 39, 5164-5180.

Elbashir, S.M., Harborth, J., Lendeckel, W., Yalcin, A., Weber, K., and Tuschl, T. (2001). Duplexes of 21-nucleotide RNAs mediate RNA interference in cultured mammalian cells. Nature 411, 494-498.

Enright, C.A., Maxwell, E.S., Eliceiri, G.L., and Sollner-Webb, B. (1996). 5'ETS rRNA processing facilitated by four small RNAs: U14, E3, U17, and U3. RNA 2, 1094-1099.

Fairman-Williams, M.E., Guenther, U.-P., and Jankowsky, E. (2010). SF1 and SF2 helicases: family matters. Curr. Opin. Struct. Biol. 20, 313-324.

Farley-Barnes, K.I., McCann, K.L., Ogawa, L.M., Merkel, J., Surovtseva, Y. V, and Baserga, S.J. (2018). Diverse Regulators of Human Ribosome Biogenesis Discovered by Changes in Nucleolar Number. Cell Rep. 22, 1923-1934.

Faza, M.B., Chang, Y., Occhipinti, L., Kemmler, S., and Panse, V.G. (2012). Role of Mex67Mtr2 in the Nuclear Export of 40S Pre-Ribosomes. PLoS Genet. 8, e1002915. 
Fedoriw, A.M., Starmer, J., Yee, D., and Magnuson, T. (2012). Nucleolar Association and Transcriptional Inhibition through 5S rDNA in Mammals. PLoS Genet. 8, e1002468.

Ferreira-Cerca, S., Pöll, G., Gleizes, P.-E., Tschochner, H., and Milkereit, P. (2005). Roles of Eukaryotic Ribosomal Proteins in Maturation and Transport of Pre-18S rRNA and Ribosome Function. Mol. Cell 20, 263-275.

Ferreira-Cerca, S., Pöll, G., Kühn, H., Neueder, A., Jakob, S., Tschochner, H., and Milkereit, P. (2007). Analysis of the In Vivo Assembly Pathway of Eukaryotic 40S Ribosomal Proteins. Mol. Cell 28, 446-457.

Fica, S.M., and Nagai, K. (2017). Cryo-electron microscopy snapshots of the spliceosome: structural insights into a dynamic ribonucleoprotein machine. Nat. Struct. Mol. Biol. 24, 791799.

Fischer, N., and Weis, K. (2002). The DEAD box protein Dhh1 stimulates the decapping enzyme Dcp1. EMBO J. 21, 2788-2797.

Flygare, J., Aspesi, A., Bailey, J.C., Miyake, K., Caffrey, J.M., Karlsson, S., and Ellis, S.R. (2006). Human RPS19, the gene mutated in Diamond-Blackfan anemia, encodes a ribosomal protein required for the maturation of 40 S ribosomal subunits. Blood 109, 980 986.

Fu, X.-D., and Ares, M. (2014). Context-dependent control of alternative splicing by RNAbinding proteins. Nat. Rev. Genet. 15, 689-701.

García-García, C., Frieda, K.L., Feoktistova, K., Fraser, C.S., and Block, S.M. (2015). RNA BIOCHEMISTRY. Factor-dependent processivity in human elF4A DEAD-box helicase. Science 348, 1486-1488.

Gartmann, M., Blau, M., Armache, J.-P., Mielke, T., Topf, M., and Beckmann, R. (2010). Mechanism of elF6-mediated Inhibition of Ribosomal Subunit Joining. J. Biol. Chem. 285, $14848-14851$.

Ghalei, H., Schaub, F.X., Doherty, J.R., Noguchi, Y., Roush, W.R., Cleveland, J.L., Stroupe, M.E., and Karbstein, K. (2015). Hrr25/CK1ס-directed release of Ltv1 from pre-40S ribosomes is necessary for ribosome assembly and cell growth. J. Cell Biol. 208, 745-759. 
Gilman, B., Tijerina, P., and Russell, R. (2017). Distinct RNA-unwinding mechanisms of DEAD-box and DEAH-box RNA helicase proteins in remodeling structured RNAs and RNPs. Biochem. Soc. Trans. 45, 1313-1321.

Gorbalenya, A.E., and Koonin, E. V. (1993). Helicases: amino acid sequence comparisons and structure-function relationships. Curr. Opin. Struct. Biol. 3, 419-429.

Gross, T., Siepmann, A., Sturm, D., Windgassen, M., Scarcelli, J.J., Seedorf, M., Cole, C.N., and Krebber, H. (2007). The DEAD-Box RNA Helicase Dbp5 Functions in Translation Termination. Science (80-. ). 315, 646-649.

Guenther, U.-P., and Jankowsky, E. (2009). Helicase Multitasking in Ribosome Assembly. Mol. Cell 36, 537-538.

Güttler, T., and Görlich, D. (2011). Ran-dependent nuclear export mediators: a structural perspective. EMBO J. 30, 3457-3474.

Haag, S., Kretschmer, J., Sloan, K.E., and Bohnsack, M.T. (2017). Crosslinking Methods to Identify RNA Methyltransferase Targets In Vivo. (Humana Press, New York, NY), pp. 269281.

Halaby, M.-J., Li, Y., Harris, B.R., Jiang, S., Miskimins, W.K., Cleary, M.P., and Yang, D.Q. (2015). Translational Control Protein 80 Stimulates IRES-Mediated Translation of p53 mRNA in Response to DNA Damage. Biomed Res. Int. 2015, 708158.

Hamann, F., Enders, M., and Ficner, R. (2019). Structural basis for RNA translocation by DEAH-box ATPases. Nucleic Acids Res. 47, 4349-4362.

He, Y., Andersen, G.R., and Nielsen, K.H. (2010). Structural basis for the function of DEAH helicases. EMBO Rep. 11, 180-186.

He, Y., Staley, J.P., Andersen, G.R., and Nielsen, K.H. (2017). Structure of the DEAH/RHA ATPase Prp43p bound to RNA implicates a pair of hairpins and motif $V a$ in translocation along RNA. RNA 23, 1110-1124.

Heininger, A.U., Hackert, P., Andreou, A.Z., Boon, K.-L., Memet, I., Prior, M., Clancy, A., Schmidt, B., Urlaub, H., Schleiff, E., et al. (2016). Protein cofactor competition regulates the 
action of a multifunctional RNA helicase in different pathways. RNA Biol. 13, 320-330.

Henras, A.K., Soudet, J., Gérus, M., Lebaron, S., Caizergues-Ferrer, M., Mougin, A., and Henry, Y. (2008). The post-transcriptional steps of eukaryotic ribosome biogenesis. Cell. Mol. Life Sci. 65, 2334-2359.

Henras, A.K., Plisson-Chastang, C., O’Donohue, M.-F., Chakraborty, A., and Gleizes, P.-E. (2015). An overview of pre-ribosomal RNA processing in eukaryotes. Wiley Interdiscip. Rev. RNA 6, 225-242.

Henras, A.K., Plisson-Chastang, C., Humbert, O., Romeo, Y., and Henry, Y. (2017). Synthesis, Function, and Heterogeneity of snoRNA-Guided Posttranscriptional Nucleoside Modifications in Eukaryotic Ribosomal RNAs. In The Enzymes, pp. 169-213.

Hilbert, M., Karow, A.R., and Klostermeier, D. (2009). The mechanism of ATP-dependent RNA unwinding by DEAD box proteins. Biol. Chem. 390, 1237-1250.

Hilbert, M., Kebbel, F., Gubaev, A., and Klostermeier, D. (2011). elF4G stimulates the activity of the DEAD box protein elF4A by a conformational guidance mechanism. Nucleic Acids Res. 39, 2260-2270.

Hilliker, A., Gao, Z., Jankowsky, E., and Parker, R. (2011). The DEAD-box protein Ded1 modulates translation by the formation and resolution of an elF4F-mRNA complex. Mol. Cell 43, 962-972.

Hooper, C., and Hilliker, A. (2013). Packing them up and dusting them off: RNA helicases and mRNA storage. Biochim. Biophys. Acta - Gene Regul. Mech. 1829, 824-834.

Hughes, J.M., and Ares, M. (1991). Depletion of U3 small nucleolar RNA inhibits cleavage in the 5' external transcribed spacer of yeast pre-ribosomal RNA and impairs formation of 18 S ribosomal RNA. EMBO J. 10, 4231-4239.

Huh, W.-K., Falvo, J. V., Gerke, L.C., Carroll, A.S., Howson, R.W., Weissman, J.S., and O'Shea, E.K. (2003). Global analysis of protein localization in budding yeast. Nature 425, 686-691.

Jagessar, K.L., and Jain, C. (2010). Functional and molecular analysis of Escherichia coli 
strains lacking multiple DEAD-box helicases. RNA 16, 1386-1392.

Jankowsky, E., and Fairman, M.E. (2007). RNA helicases — one fold for many functions. Curr. Opin. Struct. Biol. 17, 316-324.

Jarmoskaite, I., and Russell, R. (2014). RNA Helicase Proteins as Chaperones and Remodelers. Annu. Rev. Biochem. 83, 697-725.

Karginov, F. V., Caruthers, J.M., Hu, Y., McKay, D.B., and Uhlenbeck, O.C. (2005). YxiN Is a Modular Protein Combining a DEx ${ }^{\mathrm{D}} / \mathrm{H}$ Core and a Specific RNA-binding Domain. J. Biol. Chem. 280, 35499-35505.

Khajuria, R.K., Munschauer, M., Ulirsch, J.C., Panse, V.G., Carr, S.A., and Sankaran Correspondence, V.G. (2018). Ribosome Levels Selectively Regulate Translation and Lineage Commitment in Human Hematopoiesis In Brief. Cell 173, 90-103.e19.

Kiianitsa, K., Solinger, J.A., and Heyer, W.-D. (2003). NADH-coupled microplate photometric assay for kinetic studies of ATP-hydrolyzing enzymes with low and high specific activities. Anal. Biochem. 321, 266-271.

King, T.H., Liu, B., McCully, R.R., and Fournier, M.J. (2003). Ribosome structure and activity are altered in cells lacking snoRNPs that form pseudouridines in the peptidyl transferase center. Mol. Cell 11, 425-435.

Klinge, S., and Woolford, J.L. (2019). Ribosome assembly coming into focus. Nat. Rev. Mol. Cell Biol. 20, 116-131.

Klinge, S., Voigts-Hoffmann, F., Leibundgut, M., Arpagaus, S., and Ban, N. (2011). Crystal Structure of the Eukaryotic 60S Ribosomal Subunit in Complex with Initiation Factor 6. Science (80-. ). 334, 941-948.

Klinge, S., Voigts-Hoffmann, F., Leibundgut, M., and Ban, N. (2012). Atomic structures of the eukaryotic ribosome. Trends Biochem. Sci. 37, 189-198.

Knight, S., Vulliamy, T., Morgan, B., Devriendt, K., Mason, P., and Dokal, I. (2001). Identification of novel DKC1 mutations in patients with dyskeratosis congenita: implications for pathophysiology and diagnosis. Hum. Genet. 108, 299-303. 
Koš, M., and Tollervey, D. (2005). The Putative RNA Helicase Dbp4p Is Required for Release of the U14 snoRNA from Preribosomes in Saccharomyces cerevisiae. Mol. Cell $20,53-64$.

Kressler, D., Doère, M., Rojo, M., and Linder, P. (1999). Synthetic lethality with conditional dbp6 alleles identifies rsa1p, a nucleoplasmic protein involved in the assembly of $60 \mathrm{~S}$ ribosomal subunits. Mol. Cell. Biol. 19, 8633-8645.

de la Cruz, J., Kressler, D., Rojo, M., Tollervey, D., and Linder, P. (1998). Spb4p, an essential putative RNA helicase, is required for a late step in the assembly of $60 \mathrm{~S}$ ribosomal subunits in Saccharomyces cerevisiae. RNA 4, 1268-1281.

de la Cruz, J., Karbstein, K., and Woolford, J.L. (2015). Functions of Ribosomal Proteins in Assembly of Eukaryotic Ribosomes In Vivo. Annu. Rev. Biochem. 84, 93-129.

Laemmli, U.K. (1970). Cleavage of structural proteins during the assembly of the head of bacteriophage T4. Nature 227, 680-685.

Lafontaine, D.L., Preiss, T., and Tollervey, D. (1998). Yeast 18S rRNA dimethylase Dim1p: a quality control mechanism in ribosome synthesis? Mol. Cell. Biol. 18, 2360-2370.

Landry-Voyer, A.-M., Bilodeau, S., Bergeron, D., Dionne, K.L., Port, S.A., Rouleau, C., Boisvert, F.-M., Kehlenbach, R.H., and Bachand, F. (2016). Human PDCD2L Is an Export Substrate of CRM1 That Associates with 40S Ribosomal Subunit Precursors. Mol. Cell. Biol. 36, 3019-3032.

Larburu, N., Montellese, C., O'Donohue, M.-F., Kutay, U., Gleizes, P.-E., and PlissonChastang, C. (2016). Structure of a human pre-40S particle points to a role for RACK1 in the final steps of $18 \mathrm{~S}$ rRNA processing. Nucleic Acids Res. 44, 8465-8478.

Lattmann, S., Giri, B., Vaughn, J.P., Akman, S.A., and Nagamine, Y. (2010). Role of the amino terminal RHAU-specific motif in the recognition and resolution of guanine quadruplex-RNA by the DEAH-box RNA helicase RHAU. Nucleic Acids Res. 38, 62196233.

Lazdins, I.B., Delannoy, M., and Sollner-Webb, B. (1997). Analysis of nucleolar transcription and processing domains and pre-rRNA movements by in situ hybridization. Chromosoma 
$105,481-495$.

Lebaron, S., Papin, C., Capeyrou, R., Chen, Y.-L., Froment, C., Monsarrat, B., CaizerguesFerrer, M., Grigoriev, M., and Henry, Y. (2009). The ATPase and helicase activities of Prp43p are stimulated by the G-patch protein Pfa1p during yeast ribosome biogenesis. EMBO J. 28, 3808-3819.

Leeds, N.B., Small, E.C., Hiley, S.L., Hughes, T.R., and Staley, J.P. (2006). The Splicing Factor Prp43p, a DEAH Box ATPase, Functions in Ribosome Biogenesis. Mol. Cell. Biol. $26,513-522$.

Léger-Silvestre, I., Milkereit, P., Ferreira-Cerca, S., Saveanu, C., Rousselle, J.-C., Choesmel, V., Guinefoleau, C., Gas, N., and Gleizes, P.-E. (2004). The ribosomal protein Rps $15 p$ is required for nuclear exit of the $40 \mathrm{~S}$ subunit precursors in yeast. EMBO J. 23, 2336-2347.

Lewis, J.D., and Tollervey, D. (2000). Like attracts like: getting RNA processing together in the nucleus. Science 288, 1385-1389.

Li, H.D., Zagorski, J., and Fournier, M.J. (1990). Depletion of U14 small nuclear RNA (snR128) disrupts production of 18S rRNA in Saccharomyces cerevisiae. Mol. Cell. Biol. $10,1145-1152$.

Liang, W.Q., and Fournier, M.J. (1995). U14 base-pairs with 18S rRNA: a novel snoRNA interaction required for rRNA processing. Genes Dev. 9, 2433-2443.

Liang, X. -h., and Fournier, M.J. (2006). The Helicase Has1p Is Required for snoRNA Release from Pre-rRNA. Mol. Cell. Biol. 26, 7437-7450.

Lin, D., Pestova, T. V., Hellen, C.U.T., and Tiedge, H. (2008). Translational Control by a Small RNA: Dendritic BC1 RNA Targets the Eukaryotic Initiation Factor 4A Helicase Mechanism. Mol. Cell. Biol. 28, 3008.

Linder, P. (2006). Dead-box proteins: a family affair-active and passive players in RNPremodeling. Nucleic Acids Res. 34, 4168-4180.

Linder, P., and Jankowsky, E. (2011). From unwinding to clamping — the DEAD box RNA 
helicase family. Nat. Rev. Mol. Cell Biol. 12, 505-516.

Littlefield, J.W., and Dunn, D.B. (1958a). Natural occurrence of thymine and three methylated adenine bases in several ribonucleic acids. Nature 181, 254-255.

Littlefield, J.W., and Dunn, D.B. (1958b). The occurrence and distribution of thymine and three methylated-adenine bases in ribonucleic acids from several sources. Biochem. J. 70, 642-651.

Liu, J.M., and Ellis, S.R. (2006). Ribosomes and marrow failure: Coincidental association or molecular paradigm? Blood 107, 4583-4588.

Liu, F., Putnam, A., and Jankowsky, E. (2008). ATP hydrolysis is required for DEAD-box protein recycling but not for duplex unwinding. Proc. Natl. Acad. Sci. 105, 20209-20214.

Liu, Y., Deisenroth, C., and Zhang, Y. (2016). RP-MDM2-p53 Pathway: Linking Ribosomal Biogenesis and Tumor Surveillance. Trends in Cancer 2, 191-204.

Lo, K.-Y., Li, Z., Bussiere, C., Bresson, S., Marcotte, E.M., and Johnson, A.W. (2010). Defining the pathway of cytoplasmic maturation of the $60 \mathrm{~S}$ ribosomal subunit. Mol. Cell 39, 196-208.

Long, E.O., and Dawid, I.B. (1980). Repeated Genes in Eukaryotes. Annu. Rev. Biochem. $49,727-764$.

Ludwig, L.S., Gazda, H.T., Eng, J.C., Eichhorn, S.W., Thiru, P., Ghazvinian, R., George, T.I., Gotlib, J.R., Beggs, A.H., Sieff, C.A., et al. (2014). Altered translation of GATA1 in Diamond-Blackfan anemia. Nat. Med. 20, 748-753.

Luo, M.-J., Zhou, Z., Magni, K., Christoforides, C., Rappsilber, J., Mann, M., and Reed, R. (2001). Pre-mRNA splicing and mRNA export linked by direct interactions between UAP56 and Aly. Nature 413, 644-647.

Lygerou, Z., Allmang, C., Tollervey, D., and Seraphin, B. (1996). Accurate Processing of a Eukaryotic Precursor Ribosomal RNA by Ribonuclease MRP in Vitro. Science (80-. ). 272, 268-270. 
Lykke-Andersen, S., and Jensen, T.H. (2015). Nonsense-mediated mRNA decay: an intricate machinery that shapes transcriptomes. Nat. Rev. Mol. Cell Biol. 16, 665-677.

Mallam, A.L., Jarmoskaite, I., Tijerina, P., Campo, M. Del, Seifert, S., Guo, L., Russell, R., and Lambowitz, A.M. (2011). Solution structures of DEAD-box RNA chaperones reveal conformational changes and nucleic acid tethering by a basic tail. Proc. Natl. Acad. Sci. $108,12254-12259$.

Mallam, A.L., Del Campo, M., Gilman, B., Sidote, D.J., and Lambowitz, A.M. (2012). Structural basis for RNA-duplex recognition and unwinding by the DEAD-box helicase Mss116p. Nature 490, 121-125.

Malygin, A.A., Parakhnevitch, N.M., Ivanov, A. V., Eperon, I.C., and Karpova, G.G. (2007). Human ribosomal protein $\mathrm{S} 13$ regulates expression of its own gene at the splicing step by a feedback mechanism. Nucleic Acids Res. 35, 6414-6423.

Manojlovic, Z., and Stefanovic, B. (2012). A novel role of RNA helicase A in regulation of translation of type I collagen mRNAs. RNA 18, 321-334.

Martin, R., Straub, A.U., Doebele, C., and Bohnsack, M.T. (2013). DExD/H-box RNA helicases in ribosome biogenesis. RNA Biol. 10, 4-18.

Martin, R., Hackert, P., Ruprecht, M., Simm, S., Brüning, L., Mirus, O., Sloan, K.E., Kudla, G., Schleiff, E., and Bohnsack, M.T. (2014). A pre-ribosomal RNA interaction network involving snoRNAs and the Rok1 helicase. RNA 20, 1173-1182.

Matsuo, Y., Granneman, S., Thoms, M., Manikas, R.-G., Tollervey, D., and Hurt, E. (2014). Coupled GTPase and remodelling ATPase activities form a checkpoint for ribosome export. Nature 505, 112-116.

Melnikov, S., Ben-Shem, A., Garreau de Loubresse, N., Jenner, L., Yusupova, G., and Yusupov, M. (2012). One core, two shells: bacterial and eukaryotic ribosomes. Nat. Struct. Mol. Biol. 19, 560-567.

Memet, I., Doebele, C., Sloan, K.E., and Bohnsack, M.T. (2017). The G-patch protein NF$\mathrm{kB}$-repressing factor mediates the recruitment of the exonuclease XRN2 and activation of the RNA helicase DHX15 in human ribosome biogenesis. Nucleic Acids Res. 45, gkx013. 
Michelle, L., Cloutier, A., Toutant, J., Shkreta, L., Thibault, P., Durand, M., Garneau, D., Gendron, D., Lapointe, E., Couture, S., et al. (2012). Proteins associated with the exon junction complex also control the alternative splicing of apoptotic regulators. Mol. Cell. Biol. 32, 954-967.

Michot, B., Joseph, N., Mazan, S., and Bachellerie, J.P. (1999). Evolutionarily conserved structural features in the ITS2 of mammalian pre-rRNAs and potential interactions with the snoRNA U8 detected by comparative analysis of new mouse sequences. Nucleic Acids Res. 27, 2271-2282.

Mills, E.W., and Green, R. (2017). Ribosomopathies: There's strength in numbers. Science (80-. ). 358, eaan2755.

Mohr, G., Del Campo, M., Mohr, S., Yang, Q., Jia, H., Jankowsky, E., and Lambowitz, A.M. (2008). Function of the C-terminal Domain of the DEAD-box Protein Mss116p Analyzed in Vivo and in Vitro. J. Mol. Biol. 375, 1344-1364.

Montellese, C., Montel-Lehry, N., Henras, A.K., Kutay, U., Gleizes, P.-E., and O'Donohue, M.-F. (2017). Poly(A)-specific ribonuclease is a nuclear ribosome biogenesis factor involved in human $18 \mathrm{~S}$ rRNA maturation. Nucleic Acids Res. 45, 6822-6836.

Moy, R.H., Cole, B.S., Yasunaga, A., Gold, B., Shankarling, G., Varble, A., Molleston, J.M., tenOever, B.R., Lynch, K.W., and Cherry, S. (2014). Stem-Loop Recognition by DDX17 Facilitates miRNA Processing and Antiviral Defense. Cell 158, 764-777.

Mukhopadhyay, R., Jia, J., Arif, A., Ray, P.S., and Fox, P.L. (2009). The GAIT system: a gatekeeper of inflammatory gene expression. Trends Biochem. Sci. 34, 324-331.

Mullineux, S.-T., and Lafontaine, D.L.J. (2012). Mapping the cleavage sites on mammalian pre-rRNAs: where do we stand? Biochimie 94, 1521-1532.

Murzin, A.G. (1993). OB(oligonucleotide/oligosaccharide binding)-fold: common structural and functional solution for non-homologous sequences. EMBO J. 12, 861.

Naoko Tanaka, and Schwer, B. (2005). Characterization of the NTPase, RNA-Binding, and RNA Helicase Activities of the DEAH-Box Splicing Factor Prp22†. 
Napetschnig, J., Kassube, S.A., Debler, E.W., Wong, R.W., Blobel, G., and Hoelz, A. (2009). Structural and functional analysis of the interaction between the nucleoporin Nup214 and the DEAD-box helicase Ddx19. Proc. Natl. Acad. Sci. 106, 3089-3094.

Narla, A., and Ebert, B.L. (2010). Ribosomopathies: human disorders of ribosome dysfunction. Blood 115, 3196-3205.

O'Donohue, M.-F., Choesmel, V., Faubladier, M., Fichant, G., and Gleizes, P.-E. (2010). Functional dichotomy of ribosomal proteins during the synthesis of mammalian $40 \mathrm{~S}$ ribosomal subunits. J. Cell Biol. 190, 853-866.

Oeffinger, M., Dlakic, M., and Tollervey, D. (2004). A pre-ribosome-associated HEAT-repeat protein is required for export of both ribosomal subunits. Genes Dev. 18, 196-209.

Oie, S., Matsuzaki, K., Yokoyama, W., Tokunaga, S., Waku, T., Han, S.-I., Iwasaki, N., Mikogai, A., Yasuzawa-Tanaka, K., Kishimoto, H., et al. (2014). Hepatic rRNA Transcription Regulates High-Fat-Diet-Induced Obesity. Cell Rep. 7, 807-820.

Ozgur, S., Buchwald, G., Falk, S., Chakrabarti, S., Prabu, J.R., and Conti, E. (2015). The conformational plasticity of eukaryotic RNA-dependent ATPases. FEBS J. 282, 850-863.

Pause, A., and Sonenberg, N. (1992). Mutational analysis of a DEAD box RNA helicase: the mammalian translation initiation factor elF-4A. EMBO J. 11, 2643-2654.

Peculis, B.A., and Steitz, J.A. (1993). Disruption of U8 nucleolar snRNA inhibits $5.8 S$ and $28 \mathrm{~S}$ rRNA processing in the Xenopus oocyte. Cell 73, 1233-1245.

Pelava, A., Schneider, C., and Watkins, N.J. (2016). The importance of ribosome production, and the 5S RNP-MDM2 pathway, in health and disease. Biochem. Soc. Trans. 44, 1086-1090.

Pertschy, B., Saveanu, C., Zisser, G., Lebreton, A., Tengg, M., Jacquier, A., Liebminger, E., Nobis, B., Kappel, L., van der Klei, I., et al. (2007). Cytoplasmic recycling of $60 \mathrm{~S}$ preribosomal factors depends on the AAA protein Drg1. Mol. Cell. Biol. 27, 6581-6592.

Pertschy, B., Schneider, C., Gnädig, M., Schäfer, T., Tollervey, D., and Hurt, E. (2009). RNA Helicase Prp43 and Its Co-factor Pfa1 Promote 20 to 18 S rRNA Processing Catalyzed 
by the Endonuclease Nob1. J. Biol. Chem. 284, 35079-35091.

Petrov, A.S., Bernier, C.R., Hsiao, C., Norris, A.M., Kovacs, N.A., Waterbury, C.C., Stepanov, V.G., Harvey, S.C., Fox, G.E., Wartell, R.M., et al. (2014). Evolution of the ribosome at atomic resolution. Proc. Natl. Acad. Sci. U. S. A. 111, 10251-10256.

Phipps, K.R., Charette, J.M., and Baserga, S.J. (2011). The small subunit processome in ribosome biogenesis-progress and prospects. Wiley Interdiscip. Rev. RNA 2, 1-21.

Pillet, B., Mitterer, V., Kressler, D., and Pertschy, B. (2017). Hold on to your friends: Dedicated chaperones of ribosomal proteins. BioEssays 39, e201600153.

Polprasert, C., Schulze, I., Sekeres, M.A., Makishima, H., Przychodzen, B., Hosono, N., Singh, J., Padgett, R.A., Gu, X., Phillips, J.G., et al. (2015). Inherited and Somatic Defects in DDX41 in Myeloid Neoplasms. Cancer Cell 27, 658-670.

Ponting, C.P. (2000). Novel elF4G domain homologues linking mRNA translation with nonsense-mediated mRNA decay. Trends Biochem. Sci. 25, 423-426.

Preti, M., O'Donohue, M.-F., Montel-Lehry, N., Bortolin-Cavaillé, M.-L., Choesmel, V., and Gleizes, P.-E. (2013). Gradual processing of the ITS1 from the nucleolus to the cytoplasm during synthesis of the human 18S rRNA. Nucleic Acids Res. 41, 4709-4723.

Putnam, A.A., and Jankowsky, E. (2013). DEAD-box helicases as integrators of RNA, nucleotide and protein binding. Biochim. Biophys. Acta - Gene Regul. Mech. 1829, 884893.

Pyle, A.M. (2008). Translocation and Unwinding Mechanisms of RNA and DNA Helicases. Annu. Rev. Biophys. 37, 317-336.

Raman, N., Weir, E., and Müller, S. (2016). The AAA ATPase MDN1 Acts as a SUMOTargeted Regulator in Mammalian Pre-ribosome Remodeling. Mol. Cell 64, 607-615.

Robert-Paganin, J., Réty, S., and Leulliot, N. (2015a). Regulation of DEAH/RHA Helicases by G-Patch Proteins. Biomed Res. Int. 2015.

Robert-Paganin, J., Réty, S., and Leulliot, N. (2015b). Regulation of DEAH/RHA Helicases 
by G-Patch Proteins. Biomed Res. Int. 2015, 1-9.

Robledo, S., Idol, R.A., Crimmins, D.L., Ladenson, J.H., Mason, P.J., and Bessler, M. (2008). The role of human ribosomal proteins in the maturation of rRNA and ribosome production. RNA 14, 1918-1929.

Rodríguez-Galán, O., García-Gómez, J.J., and de la Cruz, J. (2013). Yeast and human RNA helicases involved in ribosome biogenesis: Current status and perspectives. Biochim. Biophys. Acta - Gene Regul. Mech. 1829, 775-790.

Rogers, G.W., Richter, N.J., and Merrick, W.C. (1999). Biochemical and Kinetic Characterization of the RNA Helicase Activity of Eukaryotic Initiation Factor 4A. J. Biol. Chem. 274, 12236-12244.

Rosado, I. V, Kressler, D., and de la Cruz, J. (2007). Functional analysis of Saccharomyces cerevisiae ribosomal protein Rpl3p in ribosome synthesis. Nucleic Acids Res. 35, 42034213.

Rudolph, M.G., and Klostermeier, D. (2015). When core competence is not enough: functional interplay of the DEAD-box helicase core with ancillary domains and auxiliary factors in RNA binding and unwinding. Biol. Chem. 396.

Sardana, R., Liu, X., Granneman, S., Zhu, J., Gill, M., Papoulas, O., Marcotte, E.M., Tollervey, D., Correll, C.C., and Johnson, A.W. (2015). The DEAH-box Helicase Dhr1 Dissociates U3 from the Pre-rRNA to Promote Formation of the Central Pseudoknot. PLOS Biol. 13, e1002083.

Schilders, G., Egberts, W.V., Raijmakers, R., and Pruijn, G.J.M. (2007). C1D is a major autoantibody target in patients with the polymyositis-scleroderma overlap syndrome. Arthritis Rheum. 56, 2449-2454.

Schillewaert, S., Wacheul, L., Lhomme, F., and Lafontaine, D.L.J. (2012). The evolutionarily conserved protein Las1 is required for pre-rRNA processing at both ends of ITS2. Mol. Cell. Biol. 32, 430-444.

Schütz, P., Bumann, M., Oberholzer, A.E., Bieniossek, C., Trachsel, H., Altmann, M., and Baumann, U. (2008). Crystal structure of the yeast elF4A-elF4G complex: an RNA-helicase 
controlled by protein-protein interactions. Proc. Natl. Acad. Sci. U. S. A. 105, 9564-9569.

Sekiguchi, T., Hayano, T., Yanagida, M., Takahashi, N., and Nishimoto, T. (2006). NOP132 is required for proper nucleolus localization of DEAD-box RNA helicase DDX47. Nucleic Acids Res. 34, 4593.

Semlow, D.R., and Staley, J.P. (2012). Staying on message: ensuring fidelity in pre-mRNA splicing. Trends Biochem. Sci. 37, 263-273.

Semlow, D.R., Blanco, M.R., Walter, N.G., and Staley, J.P. (2016). Spliceosomal DEAHBox ATPases Remodel Pre-mRNA to Activate Alternative Splice Sites. Cell 164, 985-998.

Senger, B., Lafontaine, D.L., Graindorge, J.S., Gadal, O., Camasses, A., Sanni, A., Garnier, J.M., Breitenbach, M., Hurt, E., and Fasiolo, F. (2001). The nucle(ol)ar Tif6p and Efl1p are required for a late cytoplasmic step of ribosome synthesis. Mol. Cell 8, 1363-1373.

Sentenac, A. (1985). Eukaryotic RNA polymerases. CRC Crit. Rev. Biochem. 18, 31-90.

Sharma, K., and Tollervey, D. (1999). Base pairing between U3 small nucleolar RNA and the 5 ' end of $18 \mathrm{~S}$ rRNA is required for pre-rRNA processing. Mol. Cell. Biol. 19, 6012-6019.

Sharma, S., Langhendries, J.-L., Watzinger, P., Kötter, P., Entian, K.-D., and Lafontaine, D.L.J. (2015). Yeast Kre33 and human NAT10 are conserved 18S rRNA cytosine acetyltransferases that modify tRNAs assisted by the adaptor Tan1/THUMPD1. Nucleic Acids Res. 43, 2242-2258.

SHWACHMAN, H., DIAMOND, L.K., OSKI, F.A., and KHAW, K.T. (1964). THE SYNDROME OF PANCREATIC INSUFFICIENCY AND BONE MARROW DYSFUNCTION. J. Pediatr. 65, 645-663.

Simsek, D., Tiu, G.C., Flynn, R.A., Byeon, G.W., Leppek, K., Xu, A.F., Chang, H.Y., and Barna, M. (2017). The Mammalian Ribo-interactome Reveals Ribosome Functional Diversity and Heterogeneity. Cell 169, 1051-1065.e18.

Singleton, M.R., Dillingham, M.S., and Wigley, D.B. (2007). Structure and Mechanism of Helicases and Nucleic Acid Translocases. Annu. Rev. Biochem. 76, 23-50. 
Sloan, K.E., and Bohnsack, M.T. (2018). Unravelling the Mechanisms of RNA Helicase Regulation. Trends Biochem. Sci. 43, 237-250.

Sloan, K.E., Mattijssen, S., Lebaron, S., Tollervey, D., Pruijn, G.J.M., and Watkins, N.J. (2013a). Both endonucleolytic and exonucleolytic cleavage mediate ITS1 removal during human ribosomal RNA processing. J. Cell Biol. 200, 577-588.

Sloan, K.E., Bohnsack, M.T., and Watkins, N.J. (2013b). The 5S RNP couples p53 homeostasis to ribosome biogenesis and nucleolar stress. Cell Rep. 5, 237-247.

Sloan, K.E., Bohnsack, M.T., Schneider, C., and Watkins, N.J. (2014). The roles of SSU processome components and surveillance factors in the initial processing of human ribosomal RNA. RNA 20, 540-550.

Sloan, K.E., Leisegang, M.S., Doebele, C., Ramírez, A.S., Simm, S., Safferthal, C., Kretschmer, J., Schorge, T., Markoutsa, S., Haag, S., et al. (2015). The association of lateacting snoRNPs with human pre-ribosomal complexes requires the RNA helicase DDX21. Nucleic Acids Res. 43, 553-564.

Sloan, K.E., Gleizes, P.-E., and Bohnsack, M.T. (2016). Nucleocytoplasmic Transport of RNAs and RNA-Protein Complexes. J. Mol. Biol. 428, 2040-2059.

Sloan, K.E., Warda, A.S., Sharma, S., Entian, K.-D., Lafontaine, D.L.J., and Bohnsack, M.T. (2017). Tuning the ribosome: The influence of rRNA modification on eukaryotic ribosome biogenesis and function. RNA Biol. 14, 1138-1152.

Sloan, K.E., Knox, A.A., Wells, G.R., Schneider, C., and Watkins, N.J. (2019). Interactions and activities of factors involved in the late stages of human $18 S$ rRNA maturation. RNA Biol. 16, 196-210.

Smith, J.E., Cooperman, B.S., and Mitchell, P. (1992). Methylation sites in Escherichia coli ribosomal RNA: localization and identification of four new sites of methylation in 23S rRNA. Biochemistry $31,10825-10834$.

Soltanieh, S., Osheim, Y.N., Spasov, K., Trahan, C., Beyer, A.L., and Dragon, F. (2015). DEAD-Box RNA Helicase Dbp4 Is Required for Small-Subunit Processome Formation and Function. Mol. Cell. Biol. 35, 816-830. 
Song, C., Hotz-Wagenblatt, A., Voit, R., and Grummt, I. (2017). SIRT7 and the DEAD-box helicase DDX21 cooperate to resolve genomic R loops and safeguard genome stability. Genes Dev. 31, 1370-1381.

Squatrito, M., Mancino, M., Donzelli, M., Areces, L.B., and Draetta, G.F. (2004). EBP1 is a nucleolar growth-regulating protein that is part of pre-ribosomal ribonucleoprotein complexes. Oncogene 23, 4454-4465.

Srivastava, L., Lapik, Y.R., Wang, M., and Pestov, D.G. (2010). Mammalian DEAD box protein Ddx51 acts in 3' end maturation of 28S rRNA by promoting the release of U8 snoRNA. Mol. Cell. Biol. 30, 2947-2956.

Stage-Zimmermann, T., Schmidt, U., and Silver, P.A. (2000). Factors Affecting Nuclear Export of the 60S Ribosomal Subunit In Vivo. Mol. Biol. Cell 11, 3777-3789.

Steckelberg, A.-L., Boehm, V., Gromadzka, A.M., and Gehring, N.H. (2012). CWC22 Connects Pre-mRNA Splicing and Exon Junction Complex Assembly. Cell Rep. 2, 454461.

Steitz, J.A., Berg, C., Hendrick, J.P., La Branche-Chabot, H., Metspalu, A., Rinke, J., and Yario, T. (1988). A 5S rRNA/L5 complex is a precursor to ribosome assembly in mammalian cells. J. Cell Biol. 106, 545-556.

Sun, Q., Zhu, X., Qi, J., An, W., Lan, P., Tan, D., Chen, R., Wang, B., Zheng, S., Zhang, C., et al. (2017). Molecular architecture of the $90 \mathrm{~S}$ small subunit pre-ribosome. Elife 6 .

Svitkin, Y. V, Pause, A., Haghighat, A., Pyronnet, S., Witherell, G., Belsham, G.J., and Sonenberg, N. (2001). The requirement for eukaryotic initiation factor 4A (elF4A) in translation is in direct proportion to the degree of mRNA 5' secondary structure. RNA 7, 382-394.

Tafforeau, L., Zorbas, C., Langhendries, J.-L., Mullineux, S.-T., Stamatopoulou, V., Mullier, R., Wacheul, L., and Lafontaine, D.L.J. (2013). The Complexity of Human Ribosome Biogenesis Revealed by Systematic Nucleolar Screening of Pre-rRNA Processing Factors. Mol. Cell 51, 539-551.

Takei, S., Togo-Ohno, M., Suzuki, Y., and Kuroyanagi, H. (2016). Evolutionarily conserved 
autoregulation of alternative pre-mRNA splicing by ribosomal protein L10a. Nucleic Acids Res. 44, 5585-5596.

Talkish, J., Zhang, J., Jakovljevic, J., Horsey, E.W., and Woolford, J.L. (2012). Hierarchical recruitment into nascent ribosomes of assembly factors required for 27SB pre-rRNA processing in Saccharomyces cerevisiae. Nucleic Acids Res. 40, 8646-8661.

Talwar, T., Vidhyasagar, V., Qing, J., Guo, M., Kariem, A., Lu, Y., Singh, R.S., Lukong, K.E., and $\mathrm{Wu}$, Y. (2017). The DEAD-box protein DDX43 (HAGE) is a dual RNA-DNA helicase and has a K-homology domain required for full nucleic acid unwinding activity. J. Biol. Chem. 292, 10429-10443.

Tanaka, N., Aronova, A., and Schwer, B. (2007). Ntr1 activates the Prp43 helicase to trigger release of lariat-intron from the spliceosome. Genes \&amp; Dev. 21, 2312-2325.

Tanner, N.K., Cordin, O., Banroques, J., Doère, M., and Linder, P. (2003). The Q motif: a newly identified motif in DEAD box helicases may regulate ATP binding and hydrolysis. Mol. Cell 11, 127-138.

Tauchert, M.J., Fourmann, J.-B., Lührmann, R., and Ficner, R. (2017). Structural insights into the mechanism of the DEAH-box RNA helicase Prp43. Elife 6.

Theobald, D.L., Mitton-Fry, R.M., and Wuttke, D.S. (2003). Nucleic Acid Recognition by OBFold Proteins. Annu. Rev. Biophys. Biomol. Struct. 32, 115-133.

Tieg, B., and Krebber, H. (2013). Dbp5 - From nuclear export to translation. Biochim. Biophys. Acta - Gene Regul. Mech. 1829, 791-798.

Tomecki, R., Labno, A., Drazkowska, K., Cysewski, D., and Dziembowski, A. (2015). hUTP24 is essential for processing of the human rRNA precursor at site $A_{1}$, but not at site A o. RNA Biol. 12, 1010-1029.

Trainor, P.A., Dixon, J., and Dixon, M.J. (2009). Treacher Collins syndrome: etiology, pathogenesis and prevention. Eur. J. Hum. Genet. 17, 275-283.

Turner, A.J., Knox, A.A., and Watkins, N.J. (2012). Nucleolar disruption leads to the spatial separation of key $18 \mathrm{~S}$ rRNA processing factors. RNA Biol. 9, 175-186. 
Udem, S.A., and Warner, J.R. (1972). Ribosomal RNA synthesis in Saccharomyces cerevisiae. J. Mol. Biol. 65, 227-242.

Venema, J., and Tollervey, D. (1995). Processing of pre-ribosomal RNA inSaccharomyces cerevisiae. Yeast 11, 1629-1650.

Vincent, M., Geneviève, D., Ostertag, A., Marlin, S., Lacombe, D., Martin-Coignard, D., Coubes, C., David, A., Lyonnet, S., Vilain, C., et al. (2016). Treacher Collins syndrome: a clinical and molecular study based on a large series of patients. Genet. Med. 18, 49-56.

Walbott, H., Mouffok, S., Capeyrou, R., Lebaron, S., Humbert, O., van Tilbeurgh, H., Henry, Y., and Leulliot, N. (2010). Prp43p contains a processive helicase structural architecture with a specific regulatory domain. EMBO J. 29, 2194-2204.

Walker, J.E., Saraste, M., Runswick, M.J., and Gay, N.J. (1982). Distantly related sequences in the alpha- and beta-subunits of ATP synthase, myosin, kinases and other ATP-requiring enzymes and a common nucleotide binding fold. EMBO J. 1, 945-951.

Wang, M., and Pestov, D.G. (2011). 5'-end surveillance by Xrn2 acts as a shared mechanism for mammalian pre-rRNA maturation and decay. Nucleic Acids Res. 39, 18111822.

Wang, Z., Murigneux, V., and Le Hir, H. (2014). Transcriptome-wide modulation of splicing by the exon junction complex. Genome Biol. 15, 551.

Warkocki, Z., Schneider, C., Mozaffari-Jovin, S., Schmitzová, J., Höbartner, C., Fabrizio, P., and Lührmann, R. (2015). The G-patch protein Spp2 couples the spliceosomestimulated ATPase activity of the DEAH-box protein Prp2 to catalytic activation of the spliceosome. Genes Dev. 29, 94-107.

Warner, J.R. (2001). Nascent ribosomes. Cell 107, 133-136.

Watkins, N.J., and Bohnsack, M.T. (2012). The box C/D and H/ACA snoRNPs: key players in the modification, processing and the dynamic folding of ribosomal RNA. Wiley Interdiscip. Rev. RNA 3, 397-414.

Weaver, P.L., Sun, C., and Chang, T.H. (1997). Dbp3p, a putative RNA helicase in 
Saccharomyces cerevisiae, is required for efficient pre-rRNA processing predominantly at site A3. Mol. Cell. Biol. 17, 1354-1365.

Wells, G.R., Weichmann, F., Colvin, D., Sloan, K.E., Kudla, G., Tollervey, D., Watkins, N.J., and Schneider, C. (2016). The PIN domain endonuclease Utp24 cleaves pre-ribosomal RNA at two coupled sites in yeast and humans. Nucleic Acids Res. 44, 9016-9016.

Wen, X., Tannukit, S., and Paine, M. (2008). TFIP11 Interacts with mDEAH9, an RNA Helicase Involved in Spliceosome Disassembly. Int. J. Mol. Sci. 9, 2105-2113.

Wild, T., Horvath, P., Wyler, E., Widmann, B., Badertscher, L., Zemp, I., Kozak, K., Csucs, G., Lund, E., and Kutay, U. (2010). A protein inventory of human ribosome biogenesis reveals an essential function of exportin 5 in 60S subunit export. PLoS Biol. 8, e1000522.

Witten, J.T., and Ule, J. (2011). Understanding splicing regulation through RNA splicing maps. Trends Genet. 27, 89-97.

Woolford, J.L., and Baserga, S.J. (2013). Ribosome Biogenesis in the Yeast Saccharomyces cerevisiae. Genetics 195, 643-681.

Yang, Q., Fairman, M.E., and Jankowsky, E. (2007). DEAD-box-protein-assisted RNA Structure Conversion Towards and Against Thermodynamic Equilibrium Values. J. Mol. Biol. 368, 1087-1100.

Yoneyama, M., and Fujita, T. (2008). Structural Mechanism of RNA Recognition by the RIGI-like Receptors. Immunity 29, 178-181.

Yoon, A. (2006). Impaired Control of IRES-Mediated Translation in X-Linked Dyskeratosis Congenita. Science (80-. ). 312, 902-906.

Zang, S., Lin, T.-Y., Chen, X., Gencheva, M., Newo, A.N.S., Yang, L., Rossi, D., Hu, J., Lin, S.-B., Huang, A., et al. (2014). GPKOW is essential for pre-mRNA splicing in vitro and suppresses splicing defect caused by dominant-negative DHX16 mutation in vivo. Biosci. Rep. 34, e00163.

Zemp, I., Wandrey, F., Rao, S., Ashiono, C., Wyler, E., Montellese, C., and Kutay, U. (2014). CK1 and CK1 are components of human 40 S subunit precursors required for cytoplasmic 
40 S maturation. J. Cell Sci. 127, 1242-1253.

Zhang, Y., You, J., Wang, X., and Weber, J. (2015). The DHX33 RNA Helicase Promotes mRNA Translation Initiation. Mol. Cell. Biol. 35, 2918-2931.

Zhang, Y., O'leary, M.N., Morris, D.R., Kennedy, B.K., and Wiest Correspondence, D.L. (2017). Ribosomal Proteins Rpl22 and Rpl22l1 Control Morphogenesis by Regulating PremRNA Splicing. CellReports 18, 545-556.

Zhu, J., Liu, X., Anjos, M., Correll, C.C., and Johnson, A.W. (2016). Utp14 Recruits and Activates the RNA Helicase Dhr1 To Undock U3 snoRNA from the Preribosome. Mol. Cell. Biol. 36, 965-978.

Zorbas, C., Nicolas, E., Wacheul, L., Huvelle, E., Heurgué-Hamard, V., and Lafontaine, D.L.J. (2015). The human 18S rRNA base methyltransferases DIMT1L and WBSCR22TRMT112 but not rRNA modification are required for ribosome biogenesis. Mol. Biol. Cell 26, 2080-2095. 


\section{List of Figures}

Figure1.1 Ribosome biogenesis in humans 1

Figure 1.2 Schematic representation of yeast 35 S pre-rRNA transcript...................

Figure 1.3 Overview of pre-rRNA processing in humans $\ldots \ldots \ldots \ldots \ldots \ldots \ldots \ldots \ldots \ldots \ldots \ldots$

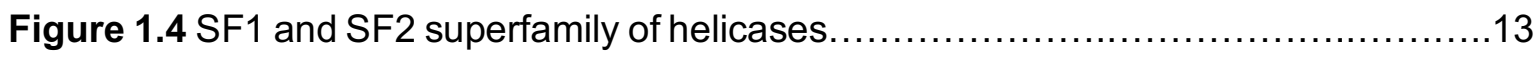

Figure 1.5 Structural characterization of DEAD-box and DEAH-box helicases...............15

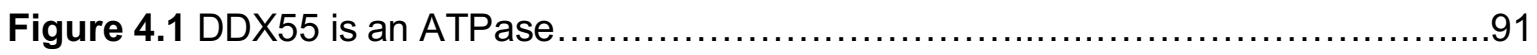

Figure 4.2 DDX55 predominantly exhibits a nucleoplasmic localisation.................92

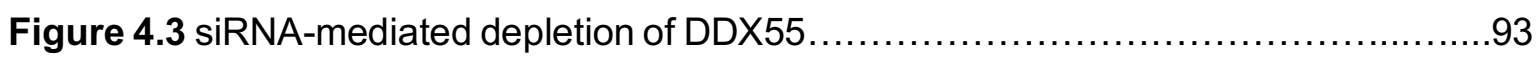

Figure 4.4 siRNA-mediated depletion of DDX55 impairs production of rRNAs..............94

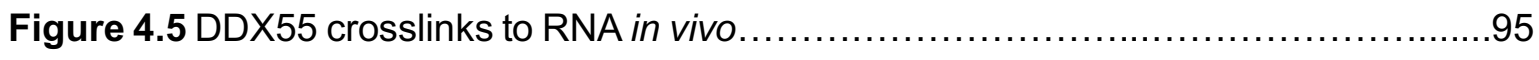

Figure 4.6 Flag-tagged DDX55 enriches ribosomal RNAs.......................................96

Figure 4.7 DDX55 crosslinks to a specific site within the 28S rRNA sequence .............97

Figure 4.8 DDX55 crosslinks to helices 62 and 63 of the $28 \mathrm{~S}$ rRNA $\ldots \ldots \ldots \ldots \ldots \ldots \ldots \ldots$

Figure 4.9 DDX55 crosslinks to helices 62 and 63 in domain IV of the $28 \mathrm{~S}$ rRNA............98

Figure 4.10 C-terminal extension of DDX55 contains conserved basic amino acid

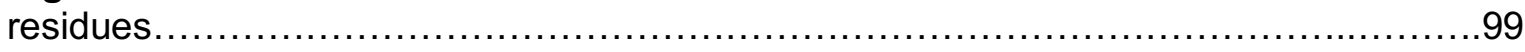

Figure 4.11 Deletion of C-terminal tail of DDX55 leads to decreased RNA binding........100

Figure 4.12 C-terminal truncation of DDX55 causes its mis-localisation to

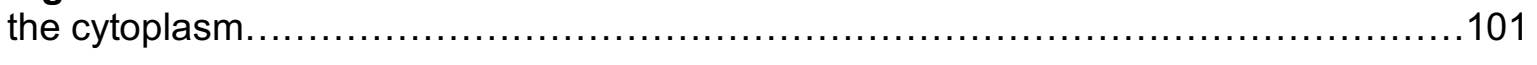

Figure 4.13 Deletion of the C-terminal basic tail of DDX55 results in reduced association

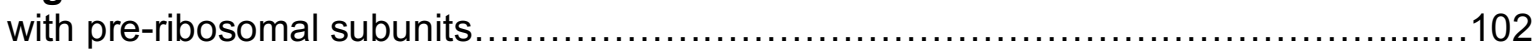




\section{List of Tables}

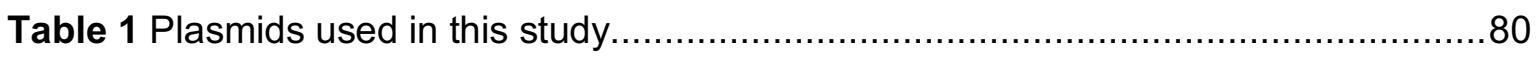

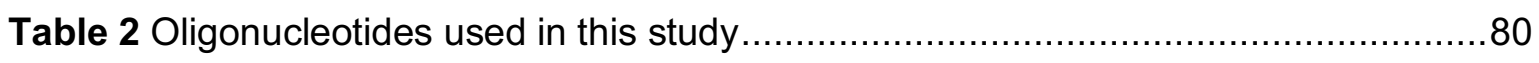

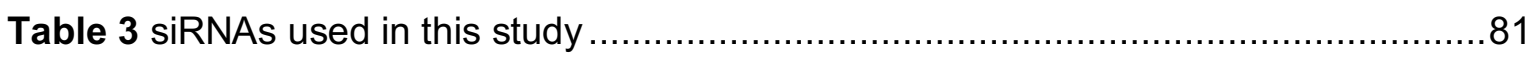

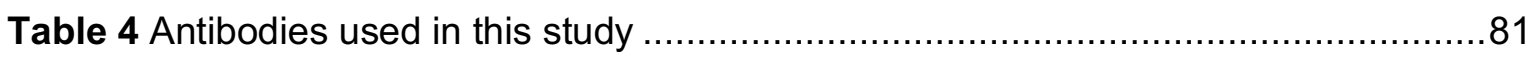

Table 5 PCR components and amplification protocol ................................................. 82

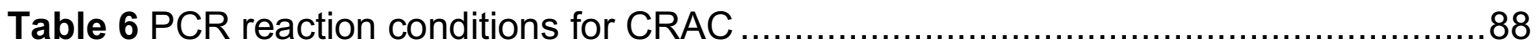




\section{Abbreviations}

\begin{tabular}{|c|c|}
\hline ATP & Adenosine triphosphate \\
\hline $\mathrm{bp}$ & Base-pair \\
\hline CRAC & Cross-linking and analysis of cDNA \\
\hline DAPI & 4',6-diamidino-2-phenylindole \\
\hline DMEM & Dulbecco's Modified Eagle Medium \\
\hline DNA & Deoxyribonucleic acid \\
\hline ETS & External transcribed spacer \\
\hline ITS & Internal transcribed spacer \\
\hline IncRNA & Long non-coding RNA \\
\hline miRNA & MicroRNA \\
\hline miscRNA & Miscellaneous RNA \\
\hline mRNA & Messenger RNA \\
\hline mtRNA & Mitochondrial RNA \\
\hline NLS & Nuclear localization signal \\
\hline $\mathrm{nt}$ & Nucleotide \\
\hline NTP & Nucleoside triphosphate \\
\hline PAGE & Polyacrylamide gel electrophoresis \\
\hline PCR & Polymerase chain reaction \\
\hline PDB & Protein data bank \\
\hline pre-mRNA & Precursor mRNA \\
\hline pre-rRNA & Precursor rRNA \\
\hline
\end{tabular}




\begin{tabular}{|c|c|}
\hline RBF & Ribosome biogenesis factor \\
\hline RNA & Ribonucleic acid \\
\hline RNP & Ribonucleoprotein \\
\hline $\mathrm{RP}$ & Ribosomal protein \\
\hline rRNA & Ribosomal RNA \\
\hline$S$ & Svedberg units \\
\hline snoRNA & Small nucleolar RNA \\
\hline snRNA & Small nuclear RNA \\
\hline SSU & Small ribosomal subunit \\
\hline tRNA & Transfer RNA \\
\hline UTR & Untranslated region \\
\hline UV & Ultraviolet \\
\hline IPTG & Isopropyl- $\beta$-D-thiogalactosidase \\
\hline $\mathrm{kDa}$ & Kilodalton \\
\hline LB & Lysogeny broth \\
\hline MIF4G & Middle domain of elF4G \\
\hline Ni-NTA & Nikel-nitrilotriacetic acid \\
\hline NT & Non-target \\
\hline siRNA & Small-interfering RNA \\
\hline OB-fold & Oligonucleotide binding fold \\
\hline$P_{i}$ & Inorganic phosphate \\
\hline RRM & RNA recognition motif \\
\hline EJC & Exon junction complex \\
\hline s.e.m & Standard error of the mean \\
\hline
\end{tabular}


SDS

SF

WH

$\mathrm{HCl}$

BSA

DAPI
Sodium dodecyl sulphate

Superfamily

Winged helix

Hydrochloric acid

Bovine serum albumin

4' 6-diamidino-2-phenylindole 


\section{Acknowledgment}

Foremost, I would like to take this opportunity to express my sincere gratitude to my supervisor Prof. Dr. Markus Bohnsack. This work would not have been possible without his support. His encouragement and unwavering motivation, despite his busy schedule, was extremely helpful during my PhD study. He also gave me the opportunity to work on various different projects and tackle novel questions which helped me immensely to expand my scientific outlook.

I would also like to thank the members of my thesis committee, Prof. Dr. Marina Rodnina and Prof. Dr. Jörg Stülke for their invaluable insights and guidance during the course of my PhD study. I would also like to thank my extended committee members: Dr. Alexander Stein, Prof. Dr. Ralf Ficner and Prof. Dr. Michael Meinecke for being a part of my examination board.

I extend my sincere thanks to Dr. Katherine Bohnsack for the constant mentorship, guidance and unending brain storming sessions. Her enthusiasm for science kept me afloat when I faced challenges during my PhD study. I am also greatly thankful to her for her critical evaluation of this dissertation.

I greatly acknowledge the help and support provided by the coordinators of International Max Planck Research School of Molecular Biology, Dr. Steffen Burkhardt and Kerstin Grüniger since the time I stepped into Göttingen.

I would also like to thank all my lab colleagues- Philipp Hackert, Ahmed Warda, Gerald Aquino, Jens Kretschmer, Lukas Brüning, Indira Memet and my lab sister Jimena for maintaining a warm and friendly working environment. I would also like to extend my gratitude to the department of molecular biology which functions as one big happy lab! I especially thank Jens Kretschmer and Philipp Hackert for their help with the CRAC experiments and bioinformatics analysis of the data.

My stay in Göttingen, this PhD and the last 11 years of my life would not have been so smooth without my sister Ridhima. Thank you, Vedran for being around and writing the thesis at the same time. It was nice sharing the struggle. A special thank you to the first friends I made in Göttingen- Kashish, Arshiya, Prajwal and Ika.

Thank you, Rahul.

Lastly, I would like to thank my family for their support and patience during the course of this study. 
Personal Born 26.02.1990 in West Bengal, India

Contact priyanka26290@gmail.com

$+49-176-68196741$

38, Hermann Rein Staße 2B

37075 Göttingen, Germany

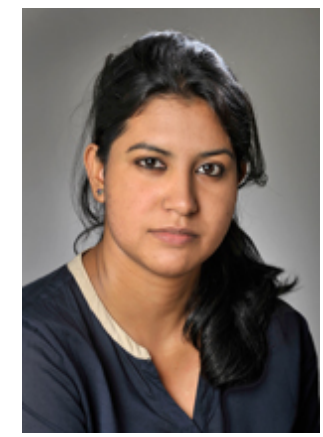

\section{Education}

2015

$\mathrm{PhD}$ candidate of Molecular Biology at University of Göttingen Dissertation titled 'Functional analyses of RNA helicases in human ribosome biogenesis'

2013

Master of Science in Molecular Biology, IMPRS Göttingen Dissertation titled 'Structural changes in elongation factor-G during translocation'

2011

Master of Science in Biochemistry, University of Delhi, India Dissertation titled' Biochemical characterization of a putative UV-B receptor from C.reinhardtii'

2008

Bachelor of Science in Biochemistry, University of Delhi, India

\section{Awards and Scholarships}

2013

Awarded Stipend of the Excellence Foundation for the promotion of the Max Planck Society

2013

Qualified National Eligibility Test for CSIR-UGC

2012

Fellowship awarded by the Indian Academy of Sciences

2012

UGC-infrastructure grant

2011

Monsanto Scholarship (awarded to top two rankers in University of Delhi) 


\section{List of publications}

Choudhury P, Hackert P, Memet I, Sloan KE, Bohnsack MT (2019). The human RNA helicase DHX37 is required for release of the U3 snoRNP from pre-ribosomal particles. RNA Biol. ;16(1): 54-68.

Sednev, M. V*., Mykhailiuk, V*., Choudhury, P.*, Halang, J., Sloan, K. E., Bohnsack, M. T., \& Höbartner, C. (2018). $\mathrm{N}^{6}$-Methyladenosine-sensitive RNA cleaving Deoxyribozymes. Angewandte Chemie; 130 (46): 15337-15341

* These authors contributed equally to this work 